\title{
Guidelines
}

\section{Responsible, Safe, and Effective Prescription of Opioids for Chronic Non-Cancer Pain: American Society of Interventional Pain Physicians (ASIPP) Guidelines}

\begin{abstract}
Laxmaiah Manchikanti, MD, Adam M. Kaye, PharmD, Nebojsa Nick Knezevic, MD, PhD, Heath McAnally, MD, Konstantin V. Slavin, MD, Andrea M. Trescot, MD, Susan Blank, MD, Vidyasagar Pampati, MSc, Salahadin Abdi, MD, PhD, Jay S. Grider, DO, PhD, Alan D. Kaye, MD, PhD, Kavita N. Manchikanti, MD, Harold J. Cordner, MD, Christopher G. Gharibo, MD, Michael E. Harned, MD, Sheri L. Albers, MD, Sairam Atluri, MD, Steve M. Aydin, DO, Sanjay Bakshi, MD, Robert Barkin, MBA, PharmD, Ramsin M. Benyamin, MD, Mark V. Boswell, MD, PhD, Ricardo M. Buenaventura, MD, Aaron K. Calodney, MD, David L. Cedeno, PhD, Sukdeb Datta, MD, Timothy R. Deer, MD, Bert Fellows, MA, Vincent Galan, MD, Vahid Grami, MD, Hans Hansen, MD, Standiford Helm II, MD, Rafael Justiz, MD, Dhanalakshmi Koyyalagunta, MD, Yogesh Malla, MD, Annu Navani, MD, Kent Nouri, MD, Ramarao Pasupuleti, MD, Nalini Sehgal, MD, Sanford M. Silverman, MD, Thomas T. Simopoulos, MD, Vijay Singh, MD, Daneshvari R. Solanki, MD, Peter S. Staats, MD, Ricardo Vallejo, MD, PhD, Bradley W. Wargo, DO, Arthur Watanabe, MD, and Joshua A. Hirsch, MD
\end{abstract}

From: American Society of Interventional Pain Physicians

Author Affiliations and Disclosures on pp. S63-S66

Address Correspondence: Laxmaiah Manchikanti, MD 2831 Lone Oak Road

Paducah, Kentucky 42003 E-mail:drlm@thepainmd.com

Manuscript received: 12-22-2016 Revised manuscript received: 01-05-2017

Accepted for publication: 02-02-2017

Free full manuscript: www.painphysicianjournal.com
Background: Opioid use, abuse, and adverse consequences, including death, have escalated at an alarming rate since the 1990s. In an attempt to control opioid abuse, numerous regulations and guidelines for responsible opioid prescribing have been developed by various organizations. However, the US opioid epidemic is continuing and drug dose deaths tripled during 1999 to 2015. Recent data show a continuing increase in deaths due to natural and semisynthetic opioids, a decline in methadone deaths, and an explosive increase in the rates of deaths involving other opioids, specifically heroin and illicit synthetic fentanyl. Contrary to scientific evidence of efficacy and negative recommendations, a significant proportion of physicians and patients (92\%) believe that opioids reduce pain and a smaller proportion (57\%) report better quality of life. In preparation of the current guidelines, we have focused on the means to reduce the abuse and diversion of opioids without jeopardizing access for those patients suffering from non-cancer pain who have an appropriate medical indication for opioid use.

Objectives: To provide guidance for the prescription of opioids for the management of chronic non-cancer pain, to develop a consistent philosophy among the many diverse groups with an interest in opioid use as to how appropriately prescribe opioids, to improve the treatment of chronic non-cancer pain and to reduce the likelihood of drug abuse and diversion. These guidelines are intended to provide a systematic and standardized approach to this complex and difficult arena of practice, while recognizing that every clinical situation is unique.

Methods: The methodology utilized included the development of objectives and key questions. The methodology also utilized trustworthy standards, appropriate disclosures of conflicts of interest, as well as a panel of experts from various specialties and groups. The literature pertaining to opioid use, abuse, effectiveness, and adverse consequences was reviewed, with a best evidence synthesis of the available literature, and utilized grading for recommendation as described by the Agency for Healthcare Research and Quality (AHRQ).

Summary of Recommendations:

i. Initial Steps of Opioid Therapy

1. Comprehensive assessment and documentation. (Evidence: Level l; Strength of Recommendation: Strong) 
2. Screening for opioid abuse to identify opioid abusers. (Evidence: Level II-III; Strength of Recommendation: Moderate)

3. Utilization of prescription drug monitoring programs (PDMPS). (Evidence: Level I-II; Strength of Recommendation: Moderate to strong)

4. Utilization of urine drug testing (UDT). (Evidence: Level II; Strength of Recommendation: Moderate)

5. Establish appropriate physical diagnosis and psychological diagnosis if available. (Evidence: Level I; Strength of Recommendation: Strong)

6. Consider appropriate imaging, physical diagnosis, and psychological status to collaborate with subjective complaints. (Evidence: Level III; Strength of Recommendation: Moderate)

7. Establish medical necessity based on average moderate to severe ( $\geq 4$ on a scale of $0-10$ ) pain and/or disability. (Evidence: Level II; Strength of Recommendation: Moderate)

8. Stratify patients based on risk. (Evidence: Level I-II; Strength of Recommendation: Moderate)

9. Establish treatment goals of opioid therapy with regard to pain relief and improvement in function. (Evidence: Level I-II; Strength of Recommendation: Moderate)

10. Obtain a robust opioid agreement, which is followed by all parties. (Evidence: Level III; Strength of Recommendation: Moderate)

ii. Assessment of Effectiveness of Long-Term Opioid Therapy

11. Initiate opioid therapy with low dose, short-acting drugs, with appropriate monitoring. (Evidence: Level II; Strength of Recommendation: Moderate)

12. Consider up to 40 morphine milligram equivalent (MME) as low dose, 41 to $90 \mathrm{MME}$ as a moderate dose, and greater than $91 \mathrm{MME}$ as high dose. (Evidence: Level II; Strength of Recommendation: Moderate)

13. Avoid long-acting opioids for the initiation of opioid therapy. (Evidence: Level I; Strength of Recommendation: Strong)

14. Recommend methadone only for use after failure of other opioid therapy and only by clinicians with specific training in its risks and uses, within FDA recommended doses. (Evidence: Level I; Strength of Recommendation: Strong)

15. Understand and educate the patients of the effectiveness and adverse consequences. (Evidence: Level I; Strength of Recommendation: Strong)

16. Similar effectiveness for long-acting and short-acting opioids with increased adverse consequences of long-acting opioids. (Evidence: Level I-II; Strength of recommendation: Moderate to strong)

17. Periodically assess pain relief and/or functional status improvement of $\geq 30 \%$ without adverse consequences. (Evidence: Level II; Strength of recommendation: Moderate)

18. Recommend long-acting or high dose opioids only in specific circumstances with severe intractable pain. (Evidence: Level I; Strength of Recommendation: Strong)

iii. Monitoring for Adherence and Side Effects

19. Monitor for adherence, abuse, and noncompliance by UDT and PDMPs. (Evidence: Level I-II; Strength of Recommendation: Moderate to strong)

20. Monitor patients on methadone with an electrocardiogram periodically. (Evidence: Level l; Strength of Recommendation: Strong).

21. Monitor for side effects including constipation and manage them appropriately, including discontinuation of opioids when indicated. (Evidence: Level l; Strength of Recommendation: Strong)

\section{iv. Final Phase}

22. May continue with monitoring with continued medical necessity, with appropriate outcomes. (Evidence: Level I-II; Strength of Recommendation: Moderate)

23. Discontinue opioid therapy for lack of response, adverse consequences, and abuse with rehabilitation. (Evidence: Level III; Strength of Recommendation: Moderate)

Conclusion: These guidelines were developed based on comprehensive review of the literature, consensus among the panelists, in consonance with patient preferences, shared decision-making, and practice patterns with limited evidence, based on randomized controlled trials (RCTs) to improve pain and function in chronic non-cancer pain on a long-term basis. Consequently, chronic opioid therapy should be provided only to patients with proven medical necessity and stability with improvement in pain and function, independently or in conjunction with other modalities of treatments in low doses with appropriate adherence monitoring and understanding of adverse events.

Key words: Chronic pain, persistent pain, non-cancer pain, controlled substances, substance abuse, prescription drug abuse, dependency, opioids, prescription monitoring, drug testing, adherence monitoring, diversion

Disclaimer: The guidelines are based on the best available evidence and do not constitute inflexible treatment recommendations. Due to the changing body of evidence, this document is not intended to be a "standard of care."

Pain Physician Opioid Special Issue 2017; 20:S3-S92 


\subsection{Introduction}

The growing epidemic of the medical use and abuse of opioid analgesics and other controlled substances is closely associated with economic burden and fatalities in the United States (1-17) and many developed countries, including Australia, Canada, and the United Kingdom (18-30) with Germany being a notable exception (31). The undertreatment of pain and the negative outcomes of unbalanced opioid policies with impaired access have been described $(19,30,32)$ and pose significant individual and public health problems in some countries, despite the markedly increased production and prescription of opioids and mounting controversies of other controlled substances $(1,18,33-42)$. This shift toward liberality in opioid prescription, however, has resulted in what are now well-documented unintended consequences $(1,12,16,41)$. The last quarter century has been marked by increasingly frequent and dramatic headline news reporting the results of overtreatment, including misuse and abuse, and deaths related to controlled substances, specifically opioids $(1,12,16,41)$.

Recent data show that the United States, with only $4.6 \%$ of the global population, used approximately $69 \%$ of the world's supply of opioids in $2014(43,44)$ including $99.7 \%$ of the hydrocodone, $51.2 \%$ of the morphine, $73.1 \%$ of the oxycodone, and $53 \%$ of the hydromorphone. Between 2000 and 2014, the United States' use of opioids increased $216 \%$ (from $46,946 \mathrm{~kg}$ to $148,316 \mathrm{~kg}$ ) whereas global use increased $210 \%$ during that period (from $69,092 \mathrm{~kg}$ to $214,490 \mathrm{~kg}$ ). Peak U.S. consumption occurred in $2012(165,525 \mathrm{~kg})$ with a $10.4 \%$ decline to $148,316 \mathrm{~kg}$ in 2014 . Driven by U.S. consumption, global figures also peaked in $2012(220,705$ $\mathrm{kg}$ ) and fell by $2.8 \%$ to $214,490 \mathrm{~kg}$ in 2014 . Since 2012 , opioid prescriptions have also taken a downturn for the first time in 2 decades, with fewer prescriptions written in 2013, 2014, and 2015. A New York Times article in May 2016 (45) reported a drop in opioid prescriptions of between $12 \%$ and $18 \%$ from $2012(46,47)$. This overall downward trend in opioid prescription has not translated to a reduction in opioid-related fatalities, however, as a paradoxical increase in deaths from heroin and synthetic illicit fentanyl $(17,36,37)$.

In 2014, opioids were involved in 28,647 deaths in the United States, with 15,559 of these deaths related to natural and semisynthetic opioids including methadone (36), increasing to 16,028 in 2015 , tripling the rate of opioid overdoses since 2000 (37). Data from the Centers for Disease Control and Prevention (CDC) show that the United States' opioid overdose epidemic includes 2 distinct but interrelated trends over the past 15 years: an increase in overdose deaths involving prescription opioid pain relievers and an explosive increase in illicit opioid (primarily heroin and illicit fentanyl) overdose deaths $(36,37)$.

While methadone deaths have received considerable attention as they comprised one-third of prescription opioid-related deaths (despite representing only $1 \%$ of opioid prescriptions), recently deaths related to methadone have declined 9.1\% from 2014 to 2015 $(36,37)$. Thus, natural and semisynthetic opioid pain relievers (e.g., morphine, codeine, oxycodone, hydrocodone, hydromorphone, and oxymorphone) continue to be involved in the majority of fatalities. While this category of opioid drug overdose deaths had declined in 2012 compared with 2011, it remained steady in 2013 with a rebound increase of $9 \%$ in 2014 and a moderate increase of $2.6 \%$ in 2015 , increasing from 12,159 to 12,727 , without the inclusion of methadone $(36,37)$. Most explosive increases have been attributed to heroin and synthetic illicit fentanyl. The $\mathrm{CDC}$ report (36) showed deaths involving methadone remained the same from 2013 to 2014, and deaths involving natural and semisynthetic opioid pain relievers, heroin, and synthetic opioids, other than methadone (i.e., fentan$\mathrm{yl}$ ), increased $9 \%, 26 \%$, and $80 \%$, respectively (Fig. 1). The CDC report of 2015 (37) was even more revealing. This report showed an increase in opioid death rates by $15.6 \%$ from 2014 to 2015 , with significant increases driven by synthetic opioids, other than methadone (i.e., fentanyl) of $72.2 \%$ and heroin of $20.6 \%$, with deaths alone due to heroin exceeding gun homicides (Figs. 2 and 3). Death rates increased in 32 states and District of Columbia and remained stable in 19 states (37).

There has been a recent worldwide focus on the development of regulations, policies, and guidelines aimed at reducing opioid misuse and abuse, primarily by improving prescription practices, without knowledge of newer developments $(36,37)$. At a governmental level, the Congress of the United States (and those of many individual states) have attempted to address the issue of the opioid epidemic by enacting various laws related to increased diversion prevention, and also by authorizing/requiring state medical boards and other entities to develop updated prescribing guidelines. However, there appears to be a disconnect between authorities, regulators, academic opponents, and practicing physicians and chronic pain patients prescribing and using opioids on a long-term basis, based on overwhelming opposition to CDC guidelines 


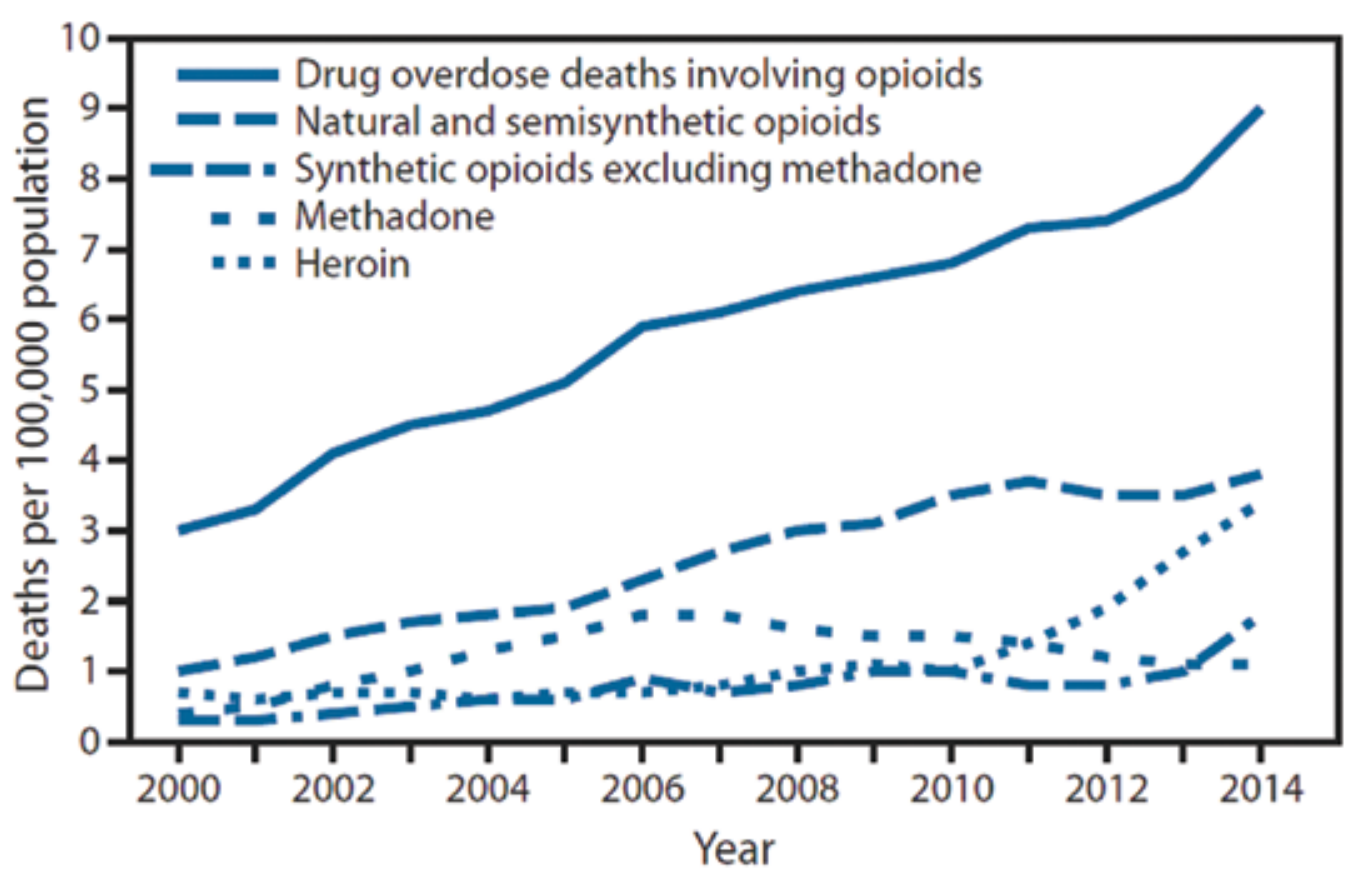

Source: National Vital Statistics System, Mortality file.

* Age-adjusted death rates were calculated by applying age-specific death rates to the 2000 U.S. standard population age distribution. $\dagger$ Drug overdose deaths involving opioids are identified using International Classification of Diseases, Tenth Revision underlying cause-ofdeath codes.

$\$$ Opioids include drugs such as morphine, oxycodone, hydrocodone, heroin, methadone, fentanyl, and tramadol. g Deaths might involve more than one drug thus categories are not exclusive.

Fig. 1. Drug overdose deaths* involving opioids, $\dagger$, $\S$ by type of opioid $\uparrow-$ United States, $2000-2014$.

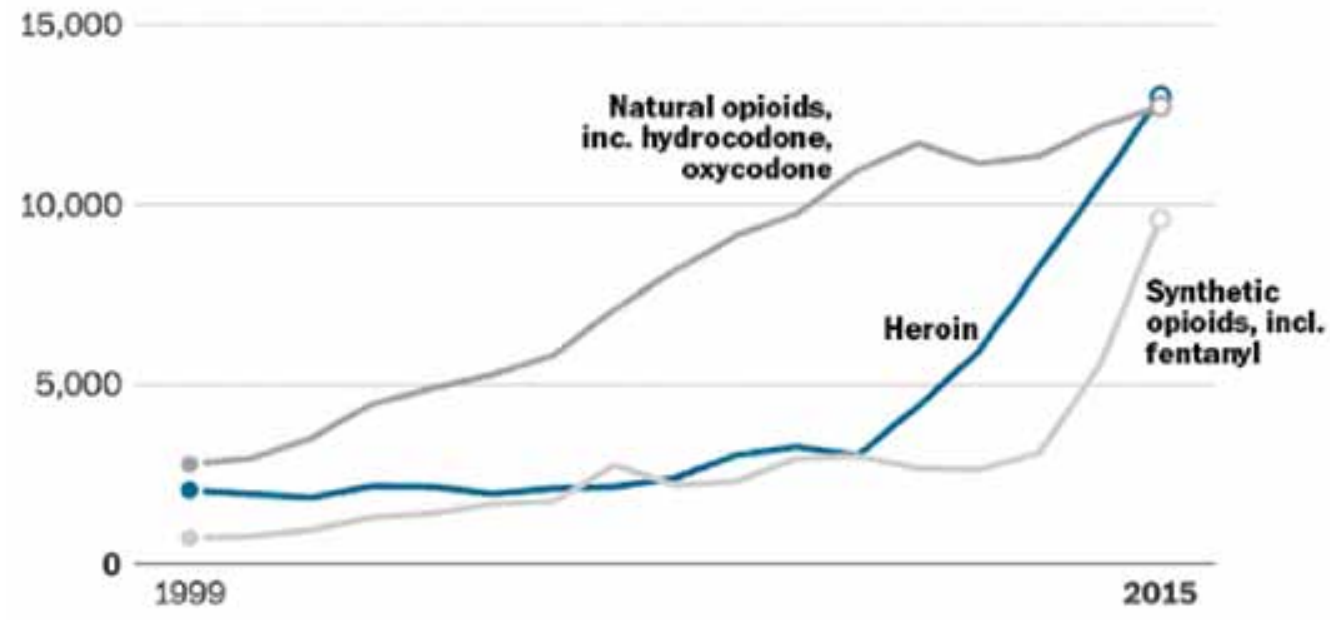

Source: CDC WONDER

Fig. 2. Heroin and fentanyl deaths rise sharply in 2015. 
Responsible, Safe, and Effective Prescription of Opioids for Chronic Non-Cancer Pain

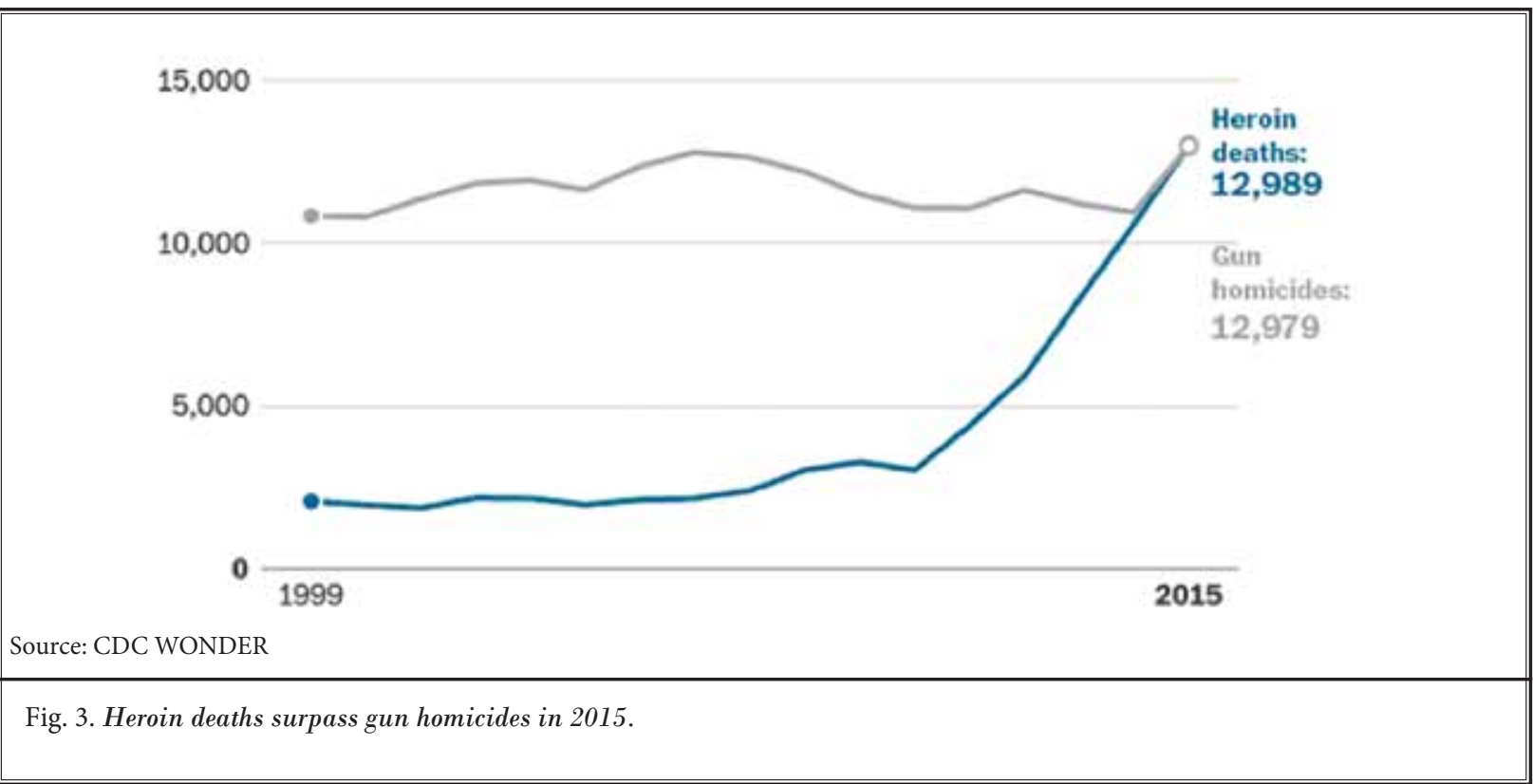

$(31,32,42)$, and Washington Post-Kaiser Family Foundation national poll of opioid users (34). While the CDC guidelines were criticized extensively by some physicians and many patients alike $(33,35)$, the Washington Post-Kaiser Family Foundation national poll of longterm opioid users showed that $92 \%$ of opioid users believe that opioids make a significant difference in their lives by reducing their pain at least somewhat well, including $53 \%$ reporting the reduction of pain as very well. Surprisingly, $57 \%$ of them stated that their quality life is better than if they had not taken the medication. This is in contrast to traditional beliefs that opioids generally reduce functional status and increase psychological distress (2-4,9-13,16,23,24,36,37,41,42). However, this survey also showed that only $20 \%$ stated that opioids have had a positive impact on their mental health, another $20 \%$ stated that they have had a negative impact on their mental health, and $60 \%$ stated there was no impact (34). The majority of the opioid users in this survey were also concerned that efforts to decrease abuse of prescription opioids could make it more difficult to obtain them. Further, the majority of them felt that prescription opioids are difficult to obtain for medical purposes.

Unfortunately, an overwhelming majority of the guidelines have not addressed critical aspects with patient perceptions, shared decision-making, and responsible, safe, and effective prescription of opioids for chronic non-cancer pain $(12,18,41)$. The major focus has been to reduce misuse and abuse primarily by improving prescription practices without consideration of subsequent consequences and newer developments in misuse and abuse.

The American Society of Interventional Pain Physicians (ASIPP) has been involved in both arenas (regulatory and advisory) since its recognition of this crisis in 2001. On the legislative front, ASIPP spearheaded the National All Schedules Prescription Electronic Reporting Act (NASPER) in 2005 (48). Many organizations including the American Medical Association (AMA) opposed this legislation (which provided for a means of national prescription monitoring capability), and NASPER was subsequently watered down to individual State Prescription Monitoring Programs with limited ability to interconnect (48). Ironically, many of these organizations formerly in opposition now support the concept and recognize the need for a national monitoring system.

From an advisory standpoint, public health agencies and institutions including the $C D C$, the Food and Drug Administration (FDA), the National Institute on Drug Abuse (NIDA), and others have also been tasked with developing guidelines and providing urgently needed opioid prescribing education (42,49-55). Professional societies, including ASIPP, have joined this urgent effort as well $(12,18,49,51,55)$. In the interest of reducing abuse and diversion, while ensuring appropriate and medically necessary prescription, the updated 


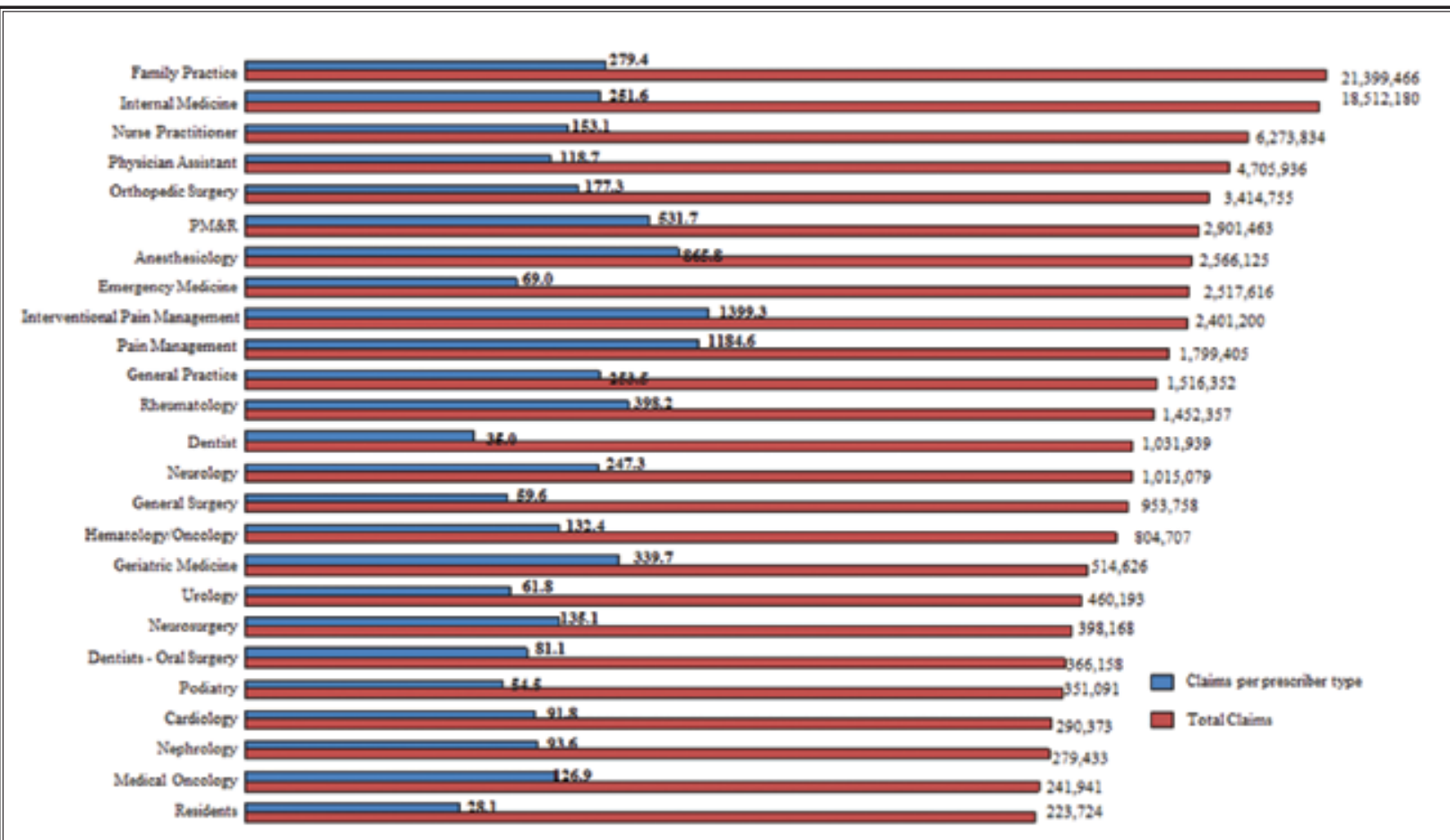

Source: https://www.cms.gov/Research-Statistics-Data-and-Systems/Statistics-Trends-and-Reports/Medicare-Provider-Charge-Data/ Part-D-Prescriber.html

Fig. 4. Top 25 prescriber specialties by total Medicare Part D claims for opioids in 2014 based on data from 2014 from Part D Prescriber Data CY 2014 (values are reported on logarithmic scale.)

American Society of Interventional Pain Physicians' Opioid Prescribing Guidelines are presented here.

In preparation for these guidelines, an epidemiologic account of the opioid epidemic was taken into consideration with the realization that while opioid prescribing by physicians and other providers appears to have unleashed the epidemic prior to 2012 $(2,4,12,16,41,52)$. It has been described that the accelerating pace of the current opioid epidemic requires a serious reconsideration of government policy initiatives that continue to focus on reductions in opioid prescribing (52). Now the focus is also directed to other factors fueling opioid overdoses and subsequent deaths including methadone prescriptions and illicit use of fentanyl and heroin.

\subsection{Methods}

\subsection{Rationale}

Pain management physicians provide a significant number of opioid and other controlled substance prescriptions for patients with chronic pain, although the specialty does not represent the highest proportion of prescribers $(12,38)$. Pain management physicians, both interventional and non-interventional (with the inclusion of anesthesiologists and physiatrists) combined provided 7.78 million prescriptions of Schedule II opioids (without the inclusion of hydrocodone) in 2013 for Medicare patients. Further, in 2014, as shown in Fig. 4, with all opioids (including of hydrocodone) combined, prescriptions of pain management and interventional pain management professionals with inclusion of an- 
esthesiologists and physiatrists increased to 9.6 million. Many physicians managing chronic pain believe that judicious and medically necessary use of opioids can be effective in controlling pain, despite the recognition of common adverse effects of physical dependence, tolerance, and addiction $(12,41)$. This concept, coincides with patient perceptions, shared decision making, and responsible, safe, and effective prescription of opioids $(12,18,41)$. Along with other clinicians, however, interventional pain physicians often are concerned about prescription opioid overuse, misuse, abuse, and adverse consequences $(1,11,12,16,18,41,48)$. The escalating prescription and use of opioids, along with increasing incidence and awareness of adverse effects, necessitates updated guidelines for opioid prescribing. These clinical practice guidelines focus on responsible, safe, and effective prescribing practices, in consonance with physician and patient preferences and shared decisionmaking $(12,32-34,42)$.

Multiple guidelines have been previously developed by various organizations $(11,12,18,49-55)$, but in some cases are incongruent and have led to discordant conclusions among various reviewers. ASIPP guidelines for responsible opioid prescribing in chronic non-cancer pain published in $2012(11,12)$ are respected by many interventional pain physicians. Since the publication of ASIPP guidelines, interagency guidelines have been updated (50) and the CDC has also recently published guidelines for primary care physicians (42).

\subsection{Objectives}

The objectives of these guidelines are to synthesize the available evidence on the comparative effectiveness and safety, as well as adverse effects of chronic opioid therapy in the treatment of chronic non-cancer pain, and provide a rational and systematic approach to their prescription. The overall goal, driven by the continuing epidemic of abuse and overdoses, is to curtail the abuse of opioids without jeopardizing appropriate non-cancer pain management with opioids.

\subsubsection{Key Questions}

These guidelines focus on the following key questions:

1. What is the impact of chronic pain on health care resources?

2. What are the statistics regarding, and trends in, opioid prescription?

3. What are the statistics regarding, and trends in, opioid misuse, abuse, and diversion?
4. What is the evidence for the therapeutic efficacy of opioids in managing chronic non-cancer pain?

5. What are the adverse consequences/harms of opioid therapy?

6. What are the best preventive and monitoring strategies to reduce or eliminate abuse while prescribing opioids?

7. What comprises responsible opioid prescribing?

8. What is the management strategy for long-term opioid therapy in chronic non-cancer pain?

\subsection{Adherence to Trustworthy Standards}

In preparation of the ASIPP guidelines for responsible opioid prescribing, the Institute of Medicine (IOM) standards and the National Guideline Clearinghouse Extent Adherence to Trustworthy Standards (NEATS) instrument were followed $(56,57)$. The NEATS instrument was developed and tested as a tool to be used by trained staff at the Agency for Healthcare Research and Quality (AHRQ) National Guideline Clearinghouse to provide assessment focused on adherence.

\subsubsection{Disclosure of Guideline Funding Source}

Responsible, safe, and effective guidelines for the prescription of opioids for chronic non-cancer pain guidelines were commissioned, prepared, edited, and endorsed by ASIPP without external funding sought or obtained. The guideline preparation committee and the writing of the guidelines were entirely supported financially by ASIPP and developed without any involvement from industry.

\subsubsection{Disclosure and Management of Financial Conflicts of Interests}

The cost of development of this guideline, including travel expenses of all panel members, was covered in full by ASIPP. Potential conflicts of interest for all panel members within the last 5 years were compiled and distributed at the introductory panel meeting. After review and discussion of these disclosures, the panel concluded that individuals with potential conflicts could remain on the panel. However, the panel members with potential conflicts were instructed by the panel and recused from related discussion or preparation of the guidelines and these members agreed not to discuss any aspect of the guidelines with the industry before data publication. Further, conflicts of interests were included on the basis of interest confluence extending beyond financial relationships including personal 
experience, practice patterns, academic interests, and promotions. Participants with previously established conflicts are considered those with opinions not being in line with previously developed ASIPP guidelines or the overall philosophical approach of ASIPP.

Disclosures and competing interests are described at the end of the manuscript.

\subsubsection{Composition of Guideline Development Group}

A large panel of experts in various medical and pharmaceutical fields, convened by ASIPP, reviewed the evidence and formulated recommendations for chronic opioid therapy in non-cancer pain. The panel consisting of authors and committee members has been instructed to assess the evidence pertaining to important aspects of opioid therapy. The panel members convened either in person or through e-seminars and telephone conferences.

The panel provided a broad representation of academic and non-academic clinical practitioners, representing a variety of specialties, disciplines, practices, and geographic areas, all with interest and expertise in opioid use and management of patients with chronic non-cancer pain.

Panel composition was multidisciplinary including methodologists (e.g., epidemiologists, statisticians, and health services researchers) with experience in research and conduct of systematic reviews. These members were voting members of the guideline development groups.

\subsubsection{Patient and Public Perspectives}

During the preparation of these guidelines, patients, patient surrogates, and members of the general public with experience in disease, opioid therapy, and complications were included. These individuals contributed by participating in completing clinical questionnaires and reviewing draft guidelines. Patient preferences were also sought and were utilized in formulating questions and in the preparation of the guidelines.

\subsubsection{Composition of Panel and Public}

Editorially, appropriate measures were taken to avoid any conflicting opinions from authors receiving funding from the industry. The panel was multidisciplinary with academicians and practitioners, and geographically diverse. Of the 48 members involved in preparing the guidelines and 45 members involved in participation through committee, there were 41 anesthesiologists, 6 physiatrists, 3 radiologists, 1 neurosurgeon, 1 internal medicine, 2 addictionologists, 1 psychiatrist, 6 pharmacists, 2 psychologists, 4 registered nurses, 1 statistician, 1 physical therapist, 3 research experts, 7 nurse practitioners, 3 physician assistants, and the remaining 7 were other professionals, either in an academic setting or in private practice. Seven patients participated in preparation of these guidelines.

\subsubsection{Evidence Review}

These guidelines were updated utilizing the evidence review, incorporating guidelines by other organizations and agencies, and developing consensus among the panel members. During that process, the panel reviewed published randomized controlled trials (RCTs) which were not included in systematic reviews, metaanalyses, narrative reviews, and clinical practice guidelines concerning the use and safety of opioid analgesics in patients with chronic non-cancer pain (57-59).

The panel updated systematic reviews from prior guideline preparation $(11,12)$, utilizing recently developed guidelines $(42,50)$ on the effectiveness and risks of long-term opioid therapy in chronic non-cancer pain and prescribing guidance, with a focus on studies addressing outcomes for long-term opioid therapy of at least one-year regarding pain, function, and quality of life (QOL). The effectiveness of short-term opioid therapy has been addressed in multiple previous studies and guidelines $(11,12,42,50)$. The guidelines also considered evidence related to initiation and titration, adverse events, and preventive strategies.

Search strategies used PubMed, Cochrane Library, Google searches, and search of websites, including the Department of Health and Human Services (HHS), the FDA, and the CDC. Search strategy terms included opioids, chronic opioid therapy in non-cancer pain, effectiveness of opioid therapy, adverse consequences, preventive strategies, monitoring, balancing opioid therapy, and abuse.

The current guidelines offer recommendations based on scientific evidence, informed expert opinion, and stakeholder input. The recommendations have been developed using principles of best evidence synthesis developed by the Cochrane Review, incorporating numerous guidelines modified by ASIPP (58).

\subsubsection{Grading or Rating the Quality or Strength of Evidence}

This grading of evidence is based on RCTs, observational studies, and other clinical reports. In addition, systematic reviews, meta-analysis, and evidence developed by other guidance are also given high importance 
with critical analysis. The grading of evidence based on ASIPP guidance is shown in Table 1.

This methodology specifies level of scientific evidence and offers a transparent approach to grading quality of evidence and strength of recommendations. The CDC has adopted the Grading of Recommendations Assessment, Development, and Evaluation method (GRADE) (42). AHRQ also has recommended similar strength of recommendation (57).

Table 1 shows the qualitative modified approach to grading of evidence providing a rating for strength of evidence, whereas Table 2 shows guidance for the strength of recommendations. Level I provides strong or significant evidence, with high confidence that the available evidence reflects the true magnitude and direction of the net effect and further research is very unlikely to change either the magnitude or direction to this net effect. Level II provides moderate or intermediate evidence with moderate confidence that the available evidence reflects the true magnitude and direction of the net effect. Thus, further research may be unlikely to alter the direction of the net effect but may alter the magnitude of the net effect.

Levels III - V provide weak evidence with low confidence that the available evidence reflects the true magnitude and direction of the net effect. Consequently, further research may change the magnitude and/or the direction of this net effect.

\subsubsection{Assessment and Recommendations of Benefits and Harms}

The guidelines intend to clearly describe the potential benefits and harms for the interventions and explicitly link the information to specific recommendations.

\subsubsection{Evidence Summary of Recommendations}

Guideline supporting documents summarize the relevant supporting evidence and explicitly link this information to recommendations.

\subsubsection{Rating or Grading the Strength of Recommendations}

IOM standards demand that for each recommendation, a rating of the strength of the recommendation in light of benefits and harms, available evidence, and the confidence in the underlying evidence should be provided. In preparation of these guidelines, the rating schemes recommended by NEATS were utilized as shown in Table 2 (57).

\subsubsection{Specificity of Recommendations}

Guideline recommendations to a major extent possible are specific and unambiguous, providing guidance on what actions should or should not be taken in various situations of chronic opioid therapy for various population groups.

Table 1. Qualitative modified approach to grading of evidence.

\begin{tabular}{|l|c|l||}
\hline Level I & Strong & $\begin{array}{l}\text { Evidence obtained from multiple relevant high quality randomized controlled trials for effectiveness } \\
\text { or } \\
\text { Evidence obtained from multiple relevant high quality observational studies or large case series for assessment of } \\
\text { preventive measures, adverse consequences, effectiveness of other measures }\end{array}$ \\
\hline Level II & Moderate & $\begin{array}{l}\text { Evidence obtained from at least one relevant high quality randomized controlled trial or multiple relevant moderate } \\
\text { or low quality randomized controlled trials } \\
\text { or } \\
\text { Evidence obtained from at least 2 high quality relevant observational studies or large case series for assessment of } \\
\text { preventive measures, adverse consequences, and effectiveness of other measures. }\end{array}$ \\
\hline Level III & Fair & $\begin{array}{l}\text { Evidence obtained from at least one relevant high quality nonrandomized trial or observational study with multiple } \\
\text { moderate or low quality observational studies } \\
\text { or } \\
\text { At least one high quality high quality relevant observational studies or large case series for assessment of preventive } \\
\text { measures, adverse consequences, effectiveness of other measures. }\end{array}$ \\
\hline Level IV & $\begin{array}{l}\text { Limited } \\
\text { Evidence obtained from multiple moderate or low quality relevant observational studies } \\
\text { Evidence obtained from moderate quality observational studies or large case series for assessment of preventive } \\
\text { measures, adverse consequences, and effectiveness of other measures. }\end{array}$ \\
\hline Level V & $\begin{array}{l}\text { Consensus } \\
\text { based }\end{array}$ & $\begin{array}{l}\text { Opinion or consensus of large group of clinicians and/or scientists for effectiveness as well as to assess preventive } \\
\text { measures, adverse consequences, effectiveness of other measures. }\end{array}$ \\
\hline
\end{tabular}

Modified from: Manchikanti et al. A modified approach to grading of evidence. Pain Physician 2014; 17:E319-E325 (58). 
Table 2. Guide for strength of recommendations.

\begin{tabular}{|l|l||}
\hline Rating for Strength of Recommendation \\
\hline Strong & $\begin{array}{l}\text { There is high confidence that the recommendation reflects best practice. This is based on: a) strong evidence for a true net } \\
\text { effect (e.g., benefits exceed harms); b) consistent results, with no or minor exceptions; c) minor or no concerns about study } \\
\text { quality; and/or d) the extent the panelists' agreement. Other compelling considerations (discussed in the guideline's literature } \\
\text { review and analyses) may also warrant a strong recommendation. }\end{array}$ \\
\hline Moderate & $\begin{array}{l}\text { There is moderate confidence that the recommendation reflects best practice. This is based on: a) good evidence for a true } \\
\text { net effect (e.g. benefits exceed harms); b) consistent results, with minor and/or few exceptions; c) minor and/or few concerns } \\
\text { about study quality; and/or d) the extent of panelists' agreement. Other compelling considerations (discussed in the guideline's } \\
\text { literature review and analyses) may also warrant a moderate recommendation. }\end{array}$ \\
\hline Weak & $\begin{array}{l}\text { There is some confidence that the recommendation offers the best current guidance for practice. This is based on: a) limited } \\
\text { evidence for a true net effect (e.g., benefits exceed harms); b) consistent results, but with important exceptions; c) concerns } \\
\text { about study quality; and/or d) the extent of panelists' agreement. Other considerations (discussed in the guideline's literature } \\
\text { review and analyses) may also warrant a weak recommendation. }\end{array}$ \\
\hline \hline
\end{tabular}

Source: National Guideline Clearinghouse Extent Adherence to Trustworthy Standards (NEATS) instrument (57).

\subsubsection{External Review}

These guidelines have been subjected to external peer review as per the policies of the publishing journal, Pain Physician. In addition, the guidelines also have been published on ASIPP's website and in their newsletter for comments from stakeholders, scientific and clinical experts, organizations, patients, and representation of the public.

\subsubsection{Updating Opioid Guidelines}

Opioid guidelines will be updated in a window of 5 years, based on significant changes in the evidence, public policy, or adverse events before January 2022.

\subsection{Impact Of Chronic Pain on Health Care: Key Question 1. What is the impact of CHRONIC PAIN ON HEALTH CARE RESOURCES?}

As illustrated by multiple reports worldwide, the impact of chronic pain is enormous (60-78). The annual U.S. expenditures alone related to pain (including direct medical costs and lost wages) by some accounts may be higher than those for cancer, heart disease, and diabetes combined. Even then, the treatment covered by these expenditures doesn't fully alleviate pain in the United States or other countries. The IOM report of 2011, despite its inaccuracies, concludes that the epidemic of chronic pain demands public health approaches with public education to counter myths, stereotypes, and stigma that hinder better care (60).

Chronic pain is defined by the International Association for the Study of Pain (IASP) as, "pain that exists beyond an expected time frame for healing" (79). However, more descriptive definitions include multiple dynamics. ASIPP has defined chronic pain as, "pain that persists 6 months after an injury and beyond the usual course of an acute disease or a reasonable time for a comparable injury to heal, that is associated with chronic pathologic processes that cause continuous or intermittent pain for months or years, that may continue in the presence or absence of demonstrable pathologies; may not be amenable to routine pain control methods; and healing may never occur" $(80,81)$.

The true burden of chronic pain has not been estimated appropriately due to variations in the chronic pain definition which includes chronic pain being present 6 months or more in the past year (70), or lasting at least 3 months (82). Further, definitions also have varied based on the severity levels (83), while others require that pain interfere with activities of daily living (82). Consequently, due to a multitude of issues, estimates of chronic pain have ranged from $11 \%$ to $55 \%$, and also have varied within countries as well $(70,82-85)$. In addition, there are multiple variations in reference to spinal pain, neuropathic pain, emotional pain, and disability (60-86).

Recent surveys show widely variable estimates. Analysis of data from a 2012 National Health Interview study revealed an estimated prevalence of daily pain of $11.2 \%$. The state of the U.S. health 1990 to 2010 describing the burden of diseases, injuries, and risk factors (85) showed that morbidity and chronic disability now account for nearly half of the U.S. health burden, with increasing life expectancy, despite substantial progress and improvement in health. Among the 30 leading diseases and injuries contributing to years lived with disability in 2010 in the United States, low back pain ranked number one, other musculoskeletal disorders ranked number 2 , neck pain ranked number 
3, major depression ranked number 4, and anxiety disorders ranked number 5 (66-68). Consequently, the top 5 conditions are primary sources of, or significantly related to, chronic pain. Studies on the global burden of disability also has estimated the point prevalence of low back pain as $9.4 \%$, with $17 \%$ of these individuals suffering from severe chronic low back pain and $25 \%$ of them suffering from severe chronic low back pain accompanied with leg pain (87). Further, in an assessment of the prevalence of neck pain with the global burden of disability has shown a point prevalence of neck pain of $4.9 \%$, with a significant proportion of patients suffering from chronic neck pain and arm pain with a high disability index (88). Chronic persistent spinal pain is reported in $25 \%$ to $60 \%$ of patients for at least one year, and even longer following an initial episode. These findings contravene the usual belief that pain is always of limited duration $(72,86)$. The estimates of regional pain in the spine also have varied with the highest prevalence in the low back of $43 \%$, followed by $32 \%$ in the neck, and the lowest prevalence in the thoracic spine (77). However, the IOM report (60), which was based on a study by Gaskin and Richard (61), rather inappropriately reported the total number of Americans suffering from chronic pain as 100 million, along with incremental medical expenditures for selected pain conditions exceeding $\$ 650$ billion, with exaggerated and dramatic numbers. However, serious flaws in this assessment have been identified with the number of people requiring treatment and the way the calculations arrived at 116 million Americans with pain requiring treatment (62). In contrast to these astounding numbers, with no scientific basis and including multiple conditions not generally considered as chronic non-cancer pain, including stroke, arthritis with surgical interventions, and spinal surgeries, others have estimated the costs of treating spinal pain, including surgical interventions (63), to be much less than $\$ 100$ billion per year. Dieleman et al (65), in an analysis of U.S. spending on personal health care and public health from 1996 to 2013 , showed an estimated spending of $\$ 87.6$ billion in managing, low back and neck pain, accounting for the third highest amount of various disease categories. They also showed that similar to the treatment of diabetes mellitus, hypertension, hyperlipidemia, depressive disorders, falls, and other neurological disorders, low back and neck pain expenditures have been increasing rapidly with an annualized increase of $6.5 \%$ from 1996 to 2013. The proportion of patients with chronic pain has been estimated to be around 30 million in the United States. Unfortunately, as has been illustrated in multiple manuscripts in the past, while the majority of the authors of the report had multiple conflicts and confluence of interest, the FDA commissioner used this data for convenience in approving potent opioids with no evidence of efficacy, safety, or need (62). As has been described, the data derived from the study from Johns Hopkins (61) misconstrued the definitions of chronic pain and consequently the resulting data.

Table 3 shows a modified presentation of the total incremental costs of medical expenditures for selected conditions as described by IOM and Gaskin and Richard (61) identifying moderate and severe pain affecting approximately 44 of 100 million people with a total expenditures of $\$ 100$ billion. As delineated in Table 4, the annual cost of chronic pain as estimated by IOM

Table 3. Total incremental costs of medical expenditures for selected pain conditions (in millions of adjusted 2010 US dollars and millions of persons).

\begin{tabular}{|l|c|c|c|}
\hline \multicolumn{1}{|c|}{ Condition } & Population (in Millions) & $\begin{array}{c}\text { Model 2 (including } \\
\text { Functional Disability) }\end{array}$ & $\begin{array}{c}\text { Model 3 (including Functional } \\
\text { Disability, Diabetes, and Asthma) }\end{array}$ \\
\hline Moderate pain & 21.3 & $\$ 39,024$ & $\$ 39,646$ \\
\hline Severe pain & 22.6 & $\$ 58,144$ & $\$ 60,000$ \\
\hline Joint pain & 70.3 & $\$ 48,280$ & $\$ 45,630$ \\
\hline Arthritis & 53.4 & $\$ 61,071$ & $\$ 59,292$ \\
\hline Functional disability & 24.7 & $\$ 93.529$ & $\$ 88,680$ \\
\hline Total & 100.0 & $\$ 300,048$ & $\$ 292,257$ \\
\hline
\end{tabular}

NOTE: Dollar amounts were adjusted for inflation as of 2010 using the Consumer Price Index Medical Care Inflation Index. This analysis is based on the total noninstitutionalized adult subpopulation of the United States for individuals aged 18 or older, who represented 210,764,398 individuals as of 2008. Model 2 includes functional disability in addition to all the other control variables. Model 3 includes functional disability, asthma, and diabetes in addition to all the other control variables. One hundred million persons had at least one of the pain conditions studied. The population total for the selected pain conditions does not sum to 100 million because some persons have multiple conditions. Source: Gaskin DJ, Richard P. The economic costs of pain in the United States. J Pain 2012; 13:715-724 (61). 
Table 4. The prevalence and cost of chronic pain.

- The annual cost of chronic pain is $\$ 560$ to $\$ 635$ billion a year
- Direct cost due to pain is $\$ 261-\$ 300$ billion
Prevalence estimates
- $10 \%$ moderate pain
- $11 \%$ severe pain
- 33\% joint pain
- $25 \%$ arthritis
- $12 \%$ functional disability
- Moderate pain $\quad \$ 4,516$
Severe pain
- Joint pain
- Arthritis

Source: Gaskin DJ, Richard P. The economic costs of pain in the United States. J Pain 2012; 13:715-724 (61).

is inaccurate with prevalence estimations of moderate and severe pain attributing to $21 \%$ of the total, the costs per individual of $\$ 4,516$ for moderate pain and $\$ 3,210$ for severe pain $(60,61)$. These estimates are similar to the estimations by Martin et al (63) with health care expenditures in the U.S. in 2005 of $\$ 86$ billion for treating back and neck pain problems. These estimates by IOM (60) also have generated significant interest in the lay press (62).

Chronic persistent pain can cause significant impairment of physical and psychological health, as well as in the performance of social responsibilities, including work and family life (60-85). Prevalence and associated disability continue to increase as illustrated by multiple studies. Freburger et al (64) showed significant and rapid increases in an evaluation in North Carolina (USA) households conducted in 1992 and repeated in 2006 showing an increase of low back pain from 3.9\% in 1992 to $10.2 \%$ in 2006. Birke et al (23) also showed an increase in chronic non-cancer pain from 18.9\% in 2000 to $26.8 \%$ in 2013 in the Danish population.

Hoy et al $(66,67)$, in a series of publications where they were evaluating low back and neck pain, showed variable prevalence with significant recurrences of $24 \%$ to $80 \%$ and increasing prevalence due to increasing age. In a comprehensive review of the epidemiology (86), the prevalence of chronic pain in the adult population has been described to range from $2 \%$ to $40 \%$, with a median point prevalence of $15 \%$. Further, the lifetime prevalence of spinal pain has been reported as $54 \%$ to $80 \%$. Studies of the prevalence of low back pain and neck pain and its impact in the general population have shown $23 \%$ of patients reporting Grade II to IV low back pain (high pain intensity with disability) versus $15 \%$ with neck pain. In addition, age-related prevalence of persistent pain appears to be much more common in the elderly associated with functional limitations and difficulty in performing daily life activities. Chronic persistent low back and neck pain is seen in $25 \%$ to $60 \%$ of the patients, one-year or longer after the initial episode (86).

In a comprehensive review of chronic non-cancer pain in Europe by Reid et al (69), the authors showed that chronic pain significantly impacted patient-perceived health status, affected everyday activities including economic pursuits and personal relationships, and was significantly associated with depressive symptoms. The one-month prevalence of moderate to severe noncancer pain was reported as $19 \%$ in this comprehensive review. Bekkering et al (68), in a systematic search of the literature and review, concluded that the prevalence of moderate to severe general chronic pain among Dutch adults was estimated at $18 \%$.

\subsection{Therapeutic Opioid Use In Managing Chronic Mon-Cancer Pain: Key Question 2. What aRE the statistics REGARDING, AND TRENDS IN, OPIOID PRESCRIPTION?}

Therapeutic opioid use, specifically in high doses, over long periods of time in chronic non-cancer pain is based on limited scientific evidence, but also is associated with serious health risks, and is based on emotion politics and economical drive to improve treatment of chronic pain $(1,4,12,16)$. Despite multiple concerns and increasing deaths, the availability and utilization of opioids has increased dramatically in the past few decades (1-42,89-128). The escalation of opioid use has been based on a single observational study published in 1986 by Portenoy and Foley looking at 38 chronic pain patients (129). In this poorly conducted retrospective review, the authors reported their experience in 38 patients maintained on opioid analgesics for nonmalignant pain. However, the most commonly used drug, hydrocodone, was not utilized, whereas, oxycodone was used by 12 patients, methadone by 7 , and levorphanol by 5 . Escalation of therapeutic opioid usage resulted in simultaneous overuse, abuse, and addiction.

Multiple reviews of trends in opioid use also have illustrated significant increases. Dart et al (2) showed that at the beginning of 2006, there were 47 million prescriptions dispensed per quarter in the United States for opioid analgesics, which peaked in the fourth quarter of 2012 at 62 million prescriptions dispensed, with an annual rate of approximately 250 million prescriptions. However, they also showed that the number of 
prescriptions trended slightly downward from 2011 through 2013. Deyo et al (3) showed an increase in opioid prescribing worldwide, with U.S. opioid sales quadrupling between 1999 and 2010. The data also showed that in 2010, among the international use of 6 powerful opioids as shown in Fig. 5, Canada topped with $753 \mathrm{MME}$ per capita with a close second by the U.S. with 693 MME per capita and a distant third place by Denmark with 470 MME per capita. Germany, Australia, United Kingdom, New Zealand, Norway, France, and Netherlands followed with 205 to 376 MME per capita. Japan scored the least with 26 MME per capita. However, this graph does not include hydrocodone, the most commonly used opioid in the United States. With the inclusion of hydrocodone, per capita use of opioids will place the United States as the number one user of global opioids. Atluri et al (1) showed the increase of medical use of all opioids from 2004 to 2011 as $65 \%$ based on the data from the Automation of Reports and Consolidated Orders System (ARCOS) with calculated grams increasing from 95 million grams to 157 million grams in 2011. They also showed that opioid use increased by $1,450 \%$ from 1996 to 2011; with increases from 1996 to 2004 of $690 \%$ and from 2004 to 2011 of $100 \%$. Opioid misuse increased approximately $4,700 \%$ from 1996 to $2011,37.70 \%$ from 1996 to 2004, and $240 \%$ from 2004 to 2011.
In the modern era of the increasing tendency of use of therapeutic interventions, prescription drug use among adults in the United States from 1999 to 2012 has increased from an estimated $51 \%$ of U.S. adults reporting use of any prescription drugs from 1999 to 2000 to an estimated $59 \%$ reporting use of any prescription drugs from 2011 to 2012, a significant increase (120). In addition, the prevalence of polypharmacy, defined as the use of at least 5 prescription drugs or more, increased from an estimated $8.2 \%$ to $15 \%$. Interestingly enough, prescription analgesics overall prevalence of use remained the same at $11 \%$ throughout the study period. However, narcotic analgesic use increased from $3.8 \%$ to $5.7 \%$. Similarly, the prescription use of anxiolytic sedatives and hypnotics also increased from $4.2 \%$ to $6.1 \%$. These data of increasing use of narcotic analgesics and anxiolytics with increasing prevalence of polypharmacy correlate with the increasing use of controlled substances.

In a prospective evaluation of psychotherapeutic and illicit drugs used by patients presenting with chronic pain at the time of their initial evaluation to an interventional pain management setting, Manchikanti et al (110), assessing the data until 2012, showed that $94 \%$ of patients were on long-term opioids. In addition, a large proportion of individuals (45.7\%) have used illicit drugs at some point in the past, with current illicit drug

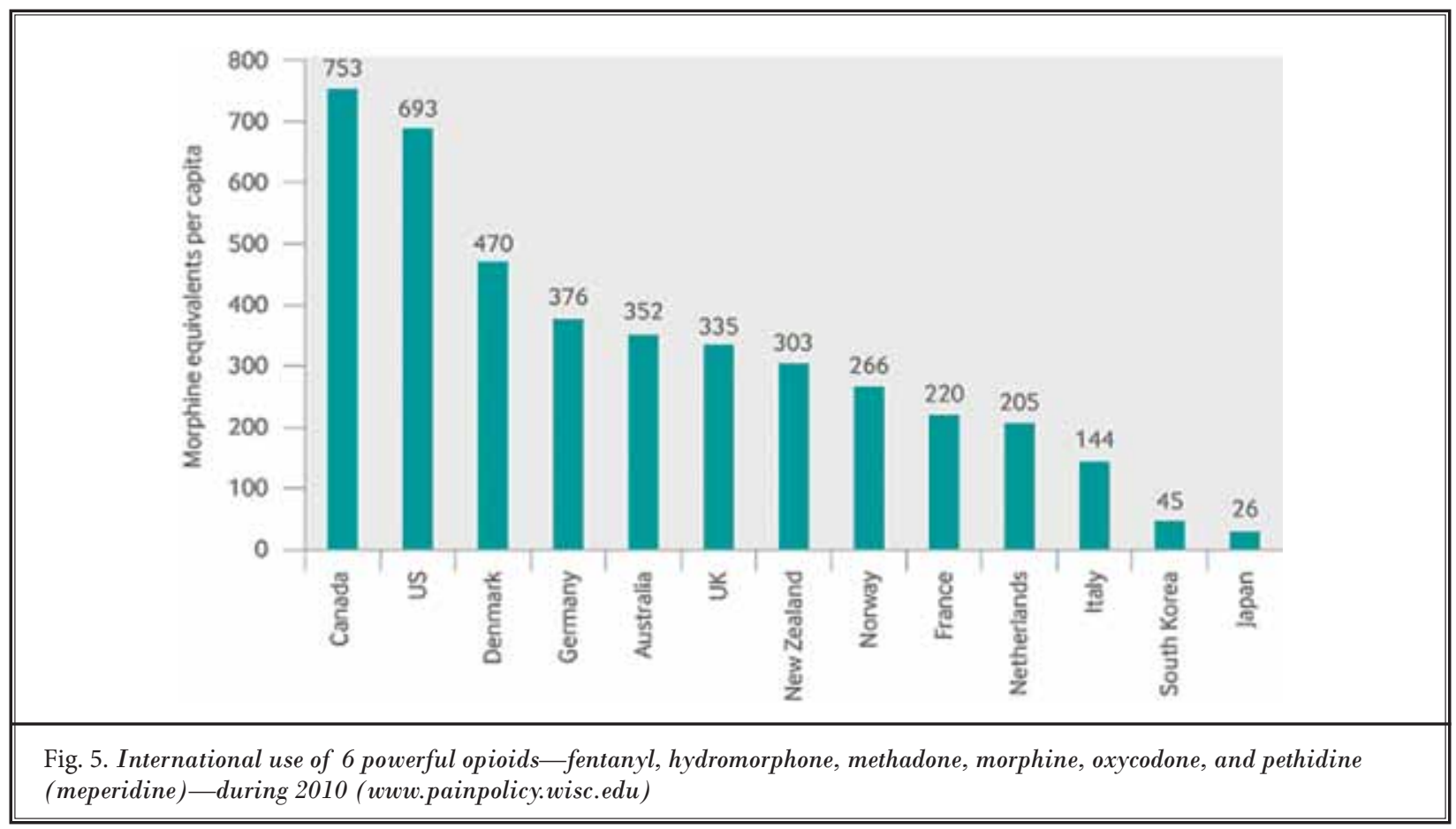

www.painphysicianjournal.com 
use present in approximately $8 \%$ of the patients. More importantly, this assessment showed combined treatment with benzodiazepines in $35 \%$ of the patients and with carisoprodol in $9 \%$ of the patients. In addition, a significant proportion of patients $(49 \%)$ have been on opioids of more than $40 \mathrm{mg}$ equivalence of morphine on a long-term basis, initiated and maintained by primary care physicians.

Jena et al (112), in a retrospective observational study of insurance claims, assessed opioid prescribing by multiple providers in Medicare in 2010 based on a large database of 1.1 million beneficiaries. The results showed that $34.6 \%$ filled prescriptions were from 2 providers, $14.2 \%$ from 3 providers, and $11.9 \%$ from 4 or more providers. This confirmed the data of prevalence of long-term use of prescription opioids among adults over the age of 65 in 2 large U.S. health care systems increasing from 5\% of patients in 1997 to $9 \%$ in 2005 (113). Morden et al (114), in an assessment of annual enrollment cohorts from 2007 to 2011, which included 6.4 million person years, assessed annual opioid use measures with chronic daily use and opioid prescriptions per user among disabled Medicare beneficiaries. This study showed that most measures peaked in 2010 with slight decreases in 2011. However, the proportion with chronic use rose from approximately $21 \%$ in 2007 to $23 \%$ in 2011 . Mean morphine equivalent dosage peaked at $81 \mathrm{mg}$ in 2010 declining to $77 \mathrm{mg}$ in 2011.

The data from workers' compensation claims is also overwhelming. A typical example of changing patterns of opioid use is from the state of Washington Workers' Compensation, Medicaid, and other insurance recipients (115). In 2008, the opioid-related mortality rate in the U.S. was 4.8 per 100,000 population, whereas the rate in Washington State was 7.4 per 100,000 population, which was approximately $60 \%$ higher $(116,117)$. The number of opioid related deaths in Washington increased from approximately 50 in 1995 to over 500 in 2008 (117). Parallel trends were evident in the Washington Workers' Compensation system, with more than 100 deaths due to accidental overdose from opioids between 2000 and 2010 (118). From 1996 to 2006, the prescriptions for Schedule II opioids tripled with mean daily doses for long-acting scheduled opioids as high as $140 \mathrm{MME}$ in 2006 (96). Following the establishment of strict guidelines in reference to opioid dosing, Garg et al (115) assessed changes in opioid prescribing patterns for chronic non-cancer pain from 2004 to 2010. The results showed a decrease of mean monthly prevalence of opioid use of $26 \%$ between 2004 and 2014 (14.4\% versus $10.7 \%$ ). Fewer incident users went on to chronic opioid therapy $(4.7 \%$ versus $6.3 \%)$. In addition, 35\% of the users were less likely to receive high doses.

Similar trends to Workers' Compensation in Washington State were also observed in Medicaid patients before and after opioid dosing guideline implementation (130). Historically, in Washington State, the number of opioid overdose deaths was nearly 6 times higher among Medicaid patients than among privately insured individuals (131). Sullivan et al (130), in an assessment of opioid dosing, showed that prescription opioid use among Washington Medicaid adults peaked in 2009, as evidenced by the approximately 105,000 opioid users and 557,000 total prescriptions. In this assessment, the median opioid dosage was unchanged from 2006 to 2010 at $37.5 \mathrm{mg}$ of morphine equivalent dose, but doses at the 75th to 99th percentile declined significantly. Garcia et al (132) compared the impact of the implementation of an opioid management initiative by the Massachusetts Medicaid pharmacy program. The calendar year 2002 was used as a base year, without any restrictions in place for members to obtain long-acting opioids, and 2005 was the comparison year, representing a time period after the multiple steps of the initiative had been implemented. Comparatively, the overall number of long-acting opioid users declined $17.8 \%$ and the overall number of claims declined by $4.1 \%$.

Patterns of opioid use for chronic non-cancer pain have been studied by the Veteran's Health Administration (VHA). Opioids are commonly prescribed for patients with chronic non-cancer pain in the Veteran's Administration (VA) population (133-136). As of October 2009, a VHA pain management directive stressed the risks associated with opioid use and mandated certain clinical changes, including the adoption of a stepped care approach, based on the biopsychosocial model, with QOL as the primary outcome (136). The number of VHA patients with chronic non-cancer pain increased slightly from 2009 to 2011 with about $50 \%$ of patients receiving at least one opioid prescription. During the study period, in each year, about $57 \%$ of those receiving opioids had at least 90 days of opioid use, which was used as an indicator of chronic opioid therapy, and $10 \%$ of individuals received opioids for at least 350 days.

The prevalence of longer term opioid use increased with age and is highest among those aged 65 and older, with $8.9 \%$ of that population taking opioid pain medications in 2013 (137). In fact, older Americans were taking opioids only for pain treatment with increasing frequen- 
cy, up $4.5 \%$ from 2009 to 2013, whereas the number of seniors using only non-steroidal anti-inflammatory drugs (NSAIDs) declined at the same time by $5.1 \%$. Further declines in NSAIDs may be seen with recent FDA warnings about NSAID use (138). In another report assessing trends and abuse and misuse of prescription opioids among older adults (121), rates of abuse and misuse of prescription opioids were lower for older patients than for younger adults; however, mortality rates among the older patients followed an increasing linear trend and surpassed rates for younger adults in 2012 and 2013. There was also an increasing linear trend among older adults, specifically with suicidal intent.

Problems similar to those in the U.S. have been described in other countries. Weisberg et al (20) shared concerns about an opioid consumption epidemic lurking at a similar level in the U.K. They showed that per capita consumption of opioids in the U.K. in 2010 was comparable to that of the U.S. in 1999, which was the beginning of a steep increase in opioid prescribing, arguably a "tipping point" in opioid misuse in the U.S. A survey of attitudes towards, and practice of, opioid prescription analgesics for chronic nonmalignant pain in general practice in the United Kingdom (137) showed almost three-quarters of general practitioners sometimes or frequently prescribed strong opioids for chronic non-cancer pain.

Canada also has been facing similar issues as the United States with increasing opioid prescriptions and related adverse consequences. Fischer et al (22) indicated that consumption of prescription opioids in Canada has steeply risen through the years 2000 to 2010 to population levels, which are second only to the United States in global comparison, with common key indicators of morbidity and mortality also increasing sharply (15).

Australia has faced significant increases in opioid prescription issues, along with their adverse consequences (29). There is ample evidence that a positive correlation exists between the magnitude of prescription opioid analgesic utilization and harms arising from both their dependence and fatal overdoses.

These data from all sources in all types of populations and most countries show increasing use of therapeutic opioids. Therapeutic opioid use has increased $210 \%$ from 2000 to 2014 globally and $216 \%$ in the United States (Tables 5 and 6). Table 7 shows the overall percent of opioid consumption in the United States compared to global supply. This has ranged for commonly used opioids from $68 \%$ to $75 \%$.

However, an analysis of consumption patterns of hydrocodone and oxycodone, 2 of the most commonly used drugs, provides a more ominous picture. Oxyco-

Table 5. Global opioid consumption in kilograms from 2000 to 2014.

\begin{tabular}{|c|c|c|c|c|c|c|c|}
\hline Year & Hydrocodone & Morphine & Oxycodone & Methadone & Fentanyl & Hydromorphone & Total* \\
\hline 2000 & 15,595 & 21,629 & 18,619 & 12,319 & 311 & 619 & 69,092 \\
\hline 2001 & 18,140 & 23,661 & 23,892 & 14,299 & 359 & 801 & 81,152 \\
\hline 2002 & 19,106 & 27,375 & 27,592 & 16,557 & 489 & 1,035 & 92,154 \\
\hline 2003 & 21,982 & 27,961 & 33,864 & 18,763 & 643 & 1,172 & 104,385 \\
\hline 2004 & 25,015 & 28,775 & 36,934 & 21,461 & 813 & 1,392 & 114,390 \\
\hline 2005 & 28,542 & 31,719 & 42,331 & 22,523 & 1,001 & 1,809 & 127,925 \\
\hline 2006 & 30,927 & 32,987 & 42,574 & 25,385 & 1,287 & 2,002 & 135,162 \\
\hline 2007 & 30,226 & 39,440 & 51,609 & 28,210 & 1,342 & 2,210 & 153,037 \\
\hline 2008 & 28,745 & 39,410 & 53,389 & 30,587 & 1,491 & 2,275 & 155,897 \\
\hline 2009 & 39,169 & 43,614 & 77,061 & 32,012 & 1,361 & 3,667 & 196,884 \\
\hline 2010 & 42,425 & 41,875 & 74,235 & 31,670 & 1,377 & 3,431 & 195,013 \\
\hline 2011 & 42,987 & 43,056 & 81,741 & 32,453 & 1,462 & 4,335 & 206,034 \\
\hline 2012 & 46,031 & 43,463 & 94,966 & 31,513 & 1,280 & 3,452 & 220,705 \\
\hline 2013 & 39,642 & 45,682 & 82,053 & 31,188 & 1,719 & 4,177 & 204,461 \\
\hline 2014 & 43,784 & 45,827 & 84,761 & 32,887 & 1,518 & 5,713 & 214,490 \\
\hline $\begin{array}{c}\text { Increase from } \\
2000-2014\end{array}$ & $180.8 \%$ & $111.9 \%$ & $355.2 \%$ & $167.0 \%$ & $388.2 \%$ & $822.9 \%$ & $210.0 \%$ \\
\hline
\end{tabular}

* Total are actual values (not morphine equivalence).

Consumption numbers for hydromorphone were gathered from yearly technical reports.

Source: https://www.incb.org/incb/en/narcotic-drugs/Technical_Reports/narcotic_drugs_reports.html 
Pain Physician: Opioid Special Issue 2017; 20:S3-S92

Table 6. U.S. opioid consumption in kilograms from 2000 to 2014.

\begin{tabular}{|c|c|c|c|c|c|c|c||}
\hline Year & Hydrocodone & Morphine & Oxycodone & Methadone & Fentanyl & Hydromorphone & $\begin{array}{c}\text { Total } \\
\text { consumption } \\
\text { in kg }\end{array}$ \\
\hline 2000 & 15,501 & 8,498 & 17,272 & 5,183 & 168 & 324 & 46,946 \\
\hline 2001 & 18,067 & 10,005 & 21,871 & 6,874 & 197 & 463 & 57,477 \\
\hline 2002 & 19,027 & 12,985 & 24,407 & 8,735 & 262 & 525 & 65,941 \\
\hline 2003 & 21,911 & 13,594 & 29,966 & 10,084 & 366 & 691 & 76,612 \\
\hline 2004 & 24,924 & 14,196 & 31,456 & 11,867 & 421 & 790 & 83,654 \\
\hline 2005 & 28,457 & 16,134 & 35,041 & 13,312 & 531 & 1000 & 94,475 \\
\hline 2006 & 30,837 & 17,355 & 34,243 & 14,774 & 627 & 1,100 & 98,936 \\
\hline 2007 & 30,147 & 23,005 & 42,445 & 15,080 & 627 & 1,200 & 112,504 \\
\hline 2008 & 28,593 & 20,550 & 40,523 & 14,846 & 722 & 994 & 106,228 \\
\hline 2009 & 39,101 & 23,403 & 62,380 & 15,324 & 583 & 2,156 & 142,947 \\
\hline 2010 & 42,355 & 22,868 & 58,987 & 15,286 & 511 & 1,900 & 141,907 \\
\hline 2011 & 42,899 & 23,099 & 66,199 & 15,289 & 573 & 2,796 & 150,855 \\
\hline 2012 & 45,976 & 24,964 & 77,405 & 15,280 & 472 & 1,428 & 165,525 \\
\hline 2013 & 39,543 & 25,571 & 63,813 & 15,787 & 539 & 2,091 & 147,344 \\
\hline 2014 & 43,649 & 23,441 & 61,921 & 15,819 & 458 & 3,028 & 148,316 \\
\hline Increase from & $181.6 \%$ & $175.8 \%$ & $258.5 \%$ & $205.2 \%$ & $172.4 \%$ & $834.6 \%$ & $178.4 \%$ \\
\hline $2000-2014$ & & & & & & \\
\hline
\end{tabular}

* Total are actual values (not morphine equivalence).

US consumption numbers for hydromorphone were gathered from yearly technical reports.

Source: https://www.incb.org/incb/en/narcotic-drugs/Technical_Reports/narcotic_drugs_reports.html

Table 7. Proportionate use of opioids in the United States compared to global consumption.

\begin{tabular}{|c|c|c|c|c|c|c|c|}
\hline & Hydrocodone & Morphine & Oxycodone & Methadone & Fentanyl & Hydromorphone & $\begin{array}{c}\text { Total } \\
\text { proportion }\end{array}$ \\
\hline 2000 & $99.4 \%$ & $39.3 \%$ & $92.8 \%$ & $42.1 \%$ & $54.0 \%$ & $52.3 \%$ & $67.9 \%$ \\
\hline 2001 & $99.6 \%$ & $42.3 \%$ & $91.5 \%$ & $48.1 \%$ & $54.9 \%$ & $57.8 \%$ & $70.8 \%$ \\
\hline 2002 & $99.6 \%$ & $47.4 \%$ & $88.5 \%$ & $52.8 \%$ & $53.6 \%$ & $50.7 \%$ & $71.6 \%$ \\
\hline 2003 & $99.7 \%$ & $48.6 \%$ & $88.5 \%$ & $53.7 \%$ & $56.9 \%$ & $59.0 \%$ & $73.4 \%$ \\
\hline 2004 & $99.6 \%$ & $49.3 \%$ & $85.2 \%$ & $55.3 \%$ & $51.8 \%$ & $56.8 \%$ & $73.1 \%$ \\
\hline 2005 & $99.7 \%$ & $50.9 \%$ & $82.8 \%$ & $59.1 \%$ & $53.0 \%$ & $55.3 \%$ & $73.9 \%$ \\
\hline 2006 & $99.7 \%$ & $52.6 \%$ & $80.4 \%$ & $58.2 \%$ & $48.7 \%$ & $54.9 \%$ & $73.2 \%$ \\
\hline 2007 & $99.7 \%$ & $58.3 \%$ & $82.2 \%$ & $53.5 \%$ & $46.7 \%$ & $54.3 \%$ & $73.5 \%$ \\
\hline 2008 & $99.5 \%$ & $52.1 \%$ & $75.9 \%$ & $48.5 \%$ & $48.4 \%$ & $43.7 \%$ & $68.1 \%$ \\
\hline 2009 & $99.8 \%$ & $53.7 \%$ & $80.9 \%$ & $47.9 \%$ & $42.8 \%$ & $58.8 \%$ & $72.6 \%$ \\
\hline 2010 & $99.8 \%$ & $54.6 \%$ & $79.5 \%$ & $48.3 \%$ & $37.1 \%$ & $55.4 \%$ & $72.8 \%$ \\
\hline 2011 & $99.8 \%$ & $53.6 \%$ & $81.0 \%$ & $47.1 \%$ & $39.2 \%$ & $64.5 \%$ & $73.2 \%$ \\
\hline 2012 & $99.9 \%$ & $57.4 \%$ & $81.5 \%$ & $48.5 \%$ & $36.8 \%$ & $41.4 \%$ & $75.0 \%$ \\
\hline 2013 & $99.8 \%$ & $56.0 \%$ & $77.8 \%$ & $50.6 \%$ & $31.4 \%$ & $50.1 \%$ & $72.1 \%$ \\
\hline 2014 & $99.7 \%$ & $51.2 \%$ & $73.1 \%$ & $48.1 \%$ & $30.1 \%$ & $53.0 \%$ & $69.1 \%$ \\
\hline
\end{tabular}

US consumption numbers for hydromorphone were gathered from yearly technical reports.

Source: https://www.incb.org/incb/en/narcotic-drugs/Technical_Reports/narcotic_drugs_reports.html 
Responsible, Safe, and Effective Prescription of Opioids for Chronic Non-Cancer Pain

done is the most commonly utilized drug in the United States and globally (based on kilogram consumption) with a $258 \%$ increase in the United States from 2000 to 2014 , compared to a $355 \%$ increase globally. However, most of the prescriptions written are for hydrocodone. Hydrocodone increased in the U.S. $181.6 \%$ and globally $180.8 \%$. The utilization of these 2 drugs in the U.S. compared to global consumption ranged from $96 \%$ in 2000 and $82 \%$ in 2014 as shown in Table 8. In addition, there also have been significant differences in overall prescription patterns with a sudden increase in utilization in kilograms from 2000 to 2014 with an overall opioid increase of $35 \%$ compared to $37 \%$ for hydrocodone and $54 \%$ for oxycodone from 2008 to 2009. Further, there also has been decreased utilization from 2012 to 2014 for all drugs. Hydrocodone during this time decreased $5 \%$, whereas oxycodone utilization decreased $25 \%$ compared to an overall decrease of all opioids of $12 \%$. The overall increase of opioid prescriptions, with the flattening or reduction since 2012 , is shown in Fig. 6. Prescriptions have increased from 1991 from 76 million to 207 million in 2013. In a report by Gusovsky (139), it was described that there were about 300 million pain prescriptions in 2015, based on specialty Pharma reports, totalling a $\$ 24$ million market. They also described that if Canada and Western Europe are included in the analysis along with the United
States, opioid consumption will increase to $95 \%$, with the remaining countries only having access to about $5 \%$ of the opioid supply. This report also showed that there was a $27 \%$ decline in the sales of hydrocodone from 2013 to 2015. This has led the Drug Enforcement Agency (DEA) to announce major cuts in opioid manufacturing with an overall $\geq 25 \%$ reduction in 2017 , with a $34 \%$ reduction for hydrocodone (140).

Opioids have been prescribed by many types of prescribers. Overall, pain physicians have been criticized for overprescriptions and increased usage of opioids. However, data show the majority of opioid prescriptions come from family physicians. In fact, in studies of the distribution of opioids by different types of Medicare prescribers (38), approximately 7.78 million prescriptions were issued by interventional pain management and pain management physicians including anesthesiologists and physiatrists. Further, in 2014, as shown in Fig. 4, with all opioids (including of hydrocodone) combined, prescriptions of pain management and interventional pain management professionals increased to 9.6 million. Family practice, internal medicine, nurse practitioners, and physician's assistants were the top 4 prescribers, followed by orthopedic surgery, physical medicine and rehabilitation, and anesthesiology specialties. However, pain management and interventional pain management professionals led the way in claims

Table 8. Consumption of hydrocodone and oxycodone in kilograms from 2000 to 2014.

\begin{tabular}{|c|c|c|c|c|c|c|c|c|c|c|c|c|}
\hline \multirow[b]{2}{*}{ Year } & \multicolumn{3}{|c|}{ Population (thousands) } & \multicolumn{3}{|c|}{ Hydrocodone (kgs) } & \multicolumn{3}{|c|}{ Oxycodone (kgs) } & \multicolumn{3}{|c|}{ Total (kgs) } \\
\hline & US. & Global & $\begin{array}{l}\% \text { over } \\
\text { Global }\end{array}$ & US & Global & $\begin{array}{l}\% \text { over } \\
\text { Global }\end{array}$ & US & Global & $\begin{array}{l}\% \text { over } \\
\text { Global }\end{array}$ & US & Global & $\begin{array}{l}\% \text { over } \\
\text { Global }\end{array}$ \\
\hline 2000 & 282,896 & $6,126,622$ & $4.6 \%$ & 15,501 & 15,595 & $99.4 \%$ & 17,272 & 18,619 & $92.8 \%$ & 32,773 & 34,214 & $95.8 \%$ \\
\hline 2001 & 285,796 & $6,204,311$ & $4.6 \%$ & 18,067 & 18,140 & $99.6 \%$ & 21,871 & 23,892 & $91.5 \%$ & 39,938 & 42,032 & $95.0 \%$ \\
\hline 2002 & 288,471 & $6,282,302$ & $4.6 \%$ & 19,027 & 19,106 & $99.6 \%$ & 24,407 & 27,592 & $88.5 \%$ & 43,434 & 46,698 & $93.0 \%$ \\
\hline 2003 & 291,005 & $6,360,765$ & $4.6 \%$ & 21,911 & 21,982 & $99.7 \%$ & 29,966 & 33,864 & $88.5 \%$ & 51,877 & 55,846 & $92.9 \%$ \\
\hline 2004 & 293,531 & $6,439,842$ & $4.6 \%$ & 24,924 & 25,015 & $99.6 \%$ & 31,456 & 36,934 & $85.2 \%$ & 56,380 & 61,949 & $91.0 \%$ \\
\hline 2005 & 296,140 & $6,519,636$ & $4.5 \%$ & 28,457 & 28,542 & $99.7 \%$ & 35,041 & 42,331 & $82.8 \%$ & 63,498 & 70,873 & $89.6 \%$ \\
\hline 2006 & 298,861 & $6,600,220$ & $4.5 \%$ & 30,837 & 30,927 & $99.7 \%$ & 34,243 & 42,574 & $80.4 \%$ & 65,080 & 73,501 & $88.5 \%$ \\
\hline 2007 & 301,656 & $6,681,607$ & $4.5 \%$ & 30,147 & 30,226 & $99.7 \%$ & 42,445 & 51,609 & $82.2 \%$ & 72,592 & 81,835 & $88.7 \%$ \\
\hline 2008 & 304,473 & $6,763,733$ & $4.5 \%$ & 28,593 & 28,745 & $99.5 \%$ & 40,523 & 53,389 & $75.9 \%$ & 69,116 & 82,134 & $84.2 \%$ \\
\hline 2009 & 307,232 & $6,846,480$ & $4.5 \%$ & 39,101 & 39,169 & $99.8 \%$ & 62,380 & 77,061 & $80.9 \%$ & 101,481 & 116,230 & $87.3 \%$ \\
\hline 2010 & 309,876 & $6,929,725$ & $4.5 \%$ & 42,355 & 42,425 & $99.8 \%$ & 58,987 & 74,235 & $79.5 \%$ & 101,342 & 116,660 & $86.9 \%$ \\
\hline 2011 & 312,390 & $7,013,427$ & $4.5 \%$ & 42,899 & 42,987 & $99.8 \%$ & 66,199 & 81,741 & $81.0 \%$ & 109,098 & 124,728 & $87.5 \%$ \\
\hline 2012 & 314,799 & $7,097,500$ & $4.4 \%$ & 45,976 & 46,031 & $99.9 \%$ & 77,405 & 94,966 & $81.5 \%$ & 123,381 & 140,997 & $87.5 \%$ \\
\hline 2013 & 317,136 & $7,181,715$ & $4.4 \%$ & 39,543 & 39,642 & $99.8 \%$ & 63,813 & 82,053 & $77.8 \%$ & 103,356 & 121,695 & $84.9 \%$ \\
\hline 2014 & 319,449 & $7,265,786$ & $4.4 \%$ & 43,649 & 43,784 & $99.7 \%$ & 61,921 & 84,761 & $73.1 \%$ & 105,570 & 128,545 & $82.1 \%$ \\
\hline
\end{tabular}




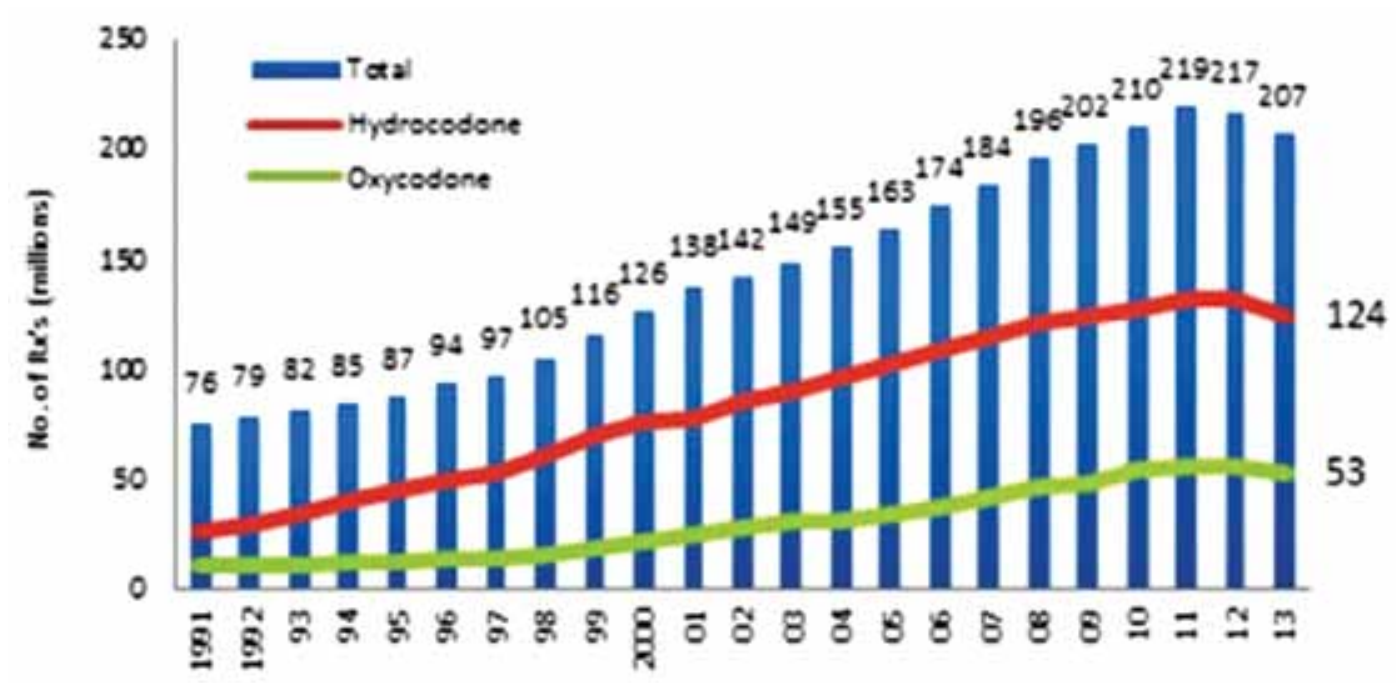

IMS Health, Vector One: National, years 1991-1996, Data Extracted 2011.

IMS Health, National Prescription Audit, years 1997-2013, Data Extracted 2014.

Fig. 6. Opioid prescriptions dispensed by US retail pharmacies from 1991 to 2013.

per prescriber type, even though other physicians led the way in number of prescriptions as shown in Fig. 4.

Concurrent use of benzodiazepines and opioids among chronic pain patients appears to be increasing gradually $(109,110,141-149)$. Overall, significant proportions ( $18 \%$ to $38 \%$ ) of chronic non-cancer patients appear to be receiving concurrent prescriptions of opioids and benzodiazepines (110,141-149). The combination of opioids and benzodiazepines has been shown to be common with increased adverse consequences. Manchikanti et al (110) have shown that $94 \%$ of patients were on long-term opioids, whereas 35\% were on benzodiazepines and $9.2 \%$ on carisoprodol prior to presenting to interventional pain management. Paulozzi et al (109) reported the results of prescription behavior surveillance systems of controlled substance prescribing patterns in 8 states in 2013. They showed that overall alprazolam, lorazepam, and clonazepam were the 3 most prescribed benzodiazepines. Multiple other studies have corroborated the significant proportion of prescriptions of benzodiazepines in chronic pain, specifically in combination with opioids. Trends of concurrent opioid analgesic and benzodiazepine use among VA patients with posttraumatic stress disorder (PTSD) from 2003 to 2011 (144) showed an approximately $53 \%$ increase, from $3.6 \%$ to $5.5 \%$, in men, and an approximately $79.5 \%$ increase, from $3.9 \%$ to $7 \%$, in women over a 9-year period. Paulozzi et al (149), in another report, also showed that in 2012, 82.5 opioid pain reliever prescriptions and 37.6 benzodiazepine prescriptions per 100,000 persons in the United States were written. There was a wide variation in the states, with a 2.7-fold for opioid pain relievers and 3.7-fold for benzodiazepines. Benzodiazepine use among chronic pain patients receiving opioids was also correlated with higher levels of pain, and physical and mental health, disability, and health service utilization (143). Further, the combination of opioids with benzodiazepines seem to be associated with substantial risks and was associated with opioid fatalities $(150,151)$. Recently, the FDA issued the requirement of a boxed warning related to the serious risks of death from combined use of opioid analgesics and benzodiazepines (152). The FDA's data review showed that physicians have been increasingly prescribing opioids and benzodiazepines together. The agency concluded that from 2000 to 2011, the rate of emergency department visits involving nonmedical use of both drug classes increased significantly, with overdose deaths involving both drug classes nearly tripling during that period. They also showed that the number of patients who were prescribed both an opioid analgesic and benzodiazepine increased by $41 \%$ between 2002 and 2014, which translated to an increase of more than 2.5 million opioid analgesic patients' receiving benzodiazepines. The use of a combination of opioids and benzodiazepines should be avoided except in medically necessary clinical settings when alternatives are not acceptable or unavailable. 


\subsection{Role of Nonmedical Use of Prescription Opioids: Key Question 3. WHAT ARE THE STATISTICS REGARDING, AND TRENDS IN, OPIOID MISUSE, ABUSE, AND DIVERSION?}

Nonmedical use of opioids is a major concern in the United States and abroad. Several terms are commonly used in the literature to describe patterns of nonmedicinal use of opioids (153). The definitions, while variable, generally describe misuse of opioids as broadly capturing any use outside of prescription parameters, including misunderstanding of instructions; self-medication of sleep, mood, or anxiety symptoms; and compulsive use driven by an opioid use disorder (153). In contrast, abuse refers to use without a prescription in a way other than prescribed, or for the experience or feelings elicited. Diversion refers to "the transfer of a controlled substance from a lawful to an unlawful channel of distribution or use, and includes both selling and giving to family members or friends for their use." Controversies ensue with the use of multiple terms describing physical dependence, tolerance, and pseudoaddiction (153).

Results of the 2014 National Survey on Drug Use and Health (NSDUH) (154) showed an estimated 27 million, or $10.2 \%$ of Americans aged 12 or older, were current (past month) illicit drug users, meaning they had used an illicit drug during the month prior to the survey interview. NSDUH in the definition of illicit drugs included marijuana/hashish, cocaine (including crack), heroin, hallucinogens, inhalants, or prescription-type psychotherapeutic (pain relievers, tranquilizers, stimulants, and sedatives) used nonmedically. These rates were higher in 2014 compared to rates from 2000 to 2013, which ranged from $7.9 \%$ to $9.2 \%$.

Among persons aged 12 or older from 2012 to 2013 who used pain relievers nonmedically in the past 12 months, $53 \%$ got the drug they used most recently from a friend or relative for free, and $10.6 \%$ bought the drug from a friend or a relative (153). In addition, $21.2 \%$ reported that they got the drug through a prescription from one doctor. Consequently, drugs purchased from a drug dealer or other strangers were only $4.3 \%$ and through internet was $0.1 \%$.

Kertesz (52), in a stimulating comment on the changing of the opioid epidemic, described that opioids commonly obtained by prescribing play a minor role, accounting for no more than $15 \%$ of reported deaths in 2015; whereas, heroin and fentanyl have come to dominate an escalating epidemic of lethal opioid overdose. Added to this is the fact that $1 \%$ of methadone prescriptions contribute to almost one-third of deaths.
Kertesz (52) described multiple shortcomings of national data including lack of current data for 2015 to 2016; lack of a unique code for fentanyl, a rising cause of overdose (155); and hindering efforts to explore the potential source of opioids that can be licitly prescribed or illicitly manufactured (155). In addition, CDC data reflect large jurisdictional inconsistencies in testing and reporting, with many local coroners not testing for fentanyl absent a reason to do so $(52,156-159)$. This is manifested in reports in Alabama by federal agencies to have only low fentanyl activity in the first half of 2014, and no activity in the latter half of 2014 (52). In contrast, Kertesz (52) has shown (Fig.78) fentanyl to be a common cause of overdose, rising from 3 deaths in 2013 to 46 for the first half of 2016 projected to be 92 per year. Heroin deaths also rose to a peak of 138 in 2014, and declined to 97 in 2015. He showed 8 deaths in which both fentanyl and heroin were causal, rising to 17 for the first 6 months of 2016. He also showed that drugs commonly obtained through prescription have declined since their peak in 2014. As shown in Fig. 7 among 30 deaths attributed to these drugs in the first half of 2016, 11 included heroin or fentanyl, with 19 of 124 drug overdose deaths, $15 \%$ of the total, in which the only identified drugs were ones commonly prescribed. However, it may also be argued that 19 of the 30 deaths $(63 \%)$ were related to prescription opioids. However, this data may not be unique to Jefferson County in Alabama, but may extend to numerous counties in the United States (159). In fact, data from Cuyahoga County (Cleveland) (156) also show that total drug overdoses as of August 31, 2016 ( $n=494$ ), exceeded the year-long total of 350 for 2015. Among these, fentanyl was found in 424, a significant increase from 92 in 2015; heroin in 350, a significant increase from 184 in 2015; and commonly prescribed opioids in 82 compared to 80 in 2015 (Fig. 8). Other data include a 3-year analysis of overdose data from Massachusetts, which found that only $8.3 \%$ of residents had a prescribed opioid at the time of their death, and $85 \%$ died due to either heroin or fentanyl.

Thus, misuse of prescription opioids appears to show a surprising and ominous pattern with escalation of opioid mortality, despite multiyear continuous reductions in opioid prescribing. In fact, the $C D C$ and DEA $(36,37)$ have described these new turns of events requiring intense attention and action. In a November 2016 report (37), the DEA referred to heroin and fentanyl as the most significant drug-related threats to the United States (Figs. 1-4). Unfortunately, despite declining methadone deaths, a much smaller increase $(2.6 \%)$ 


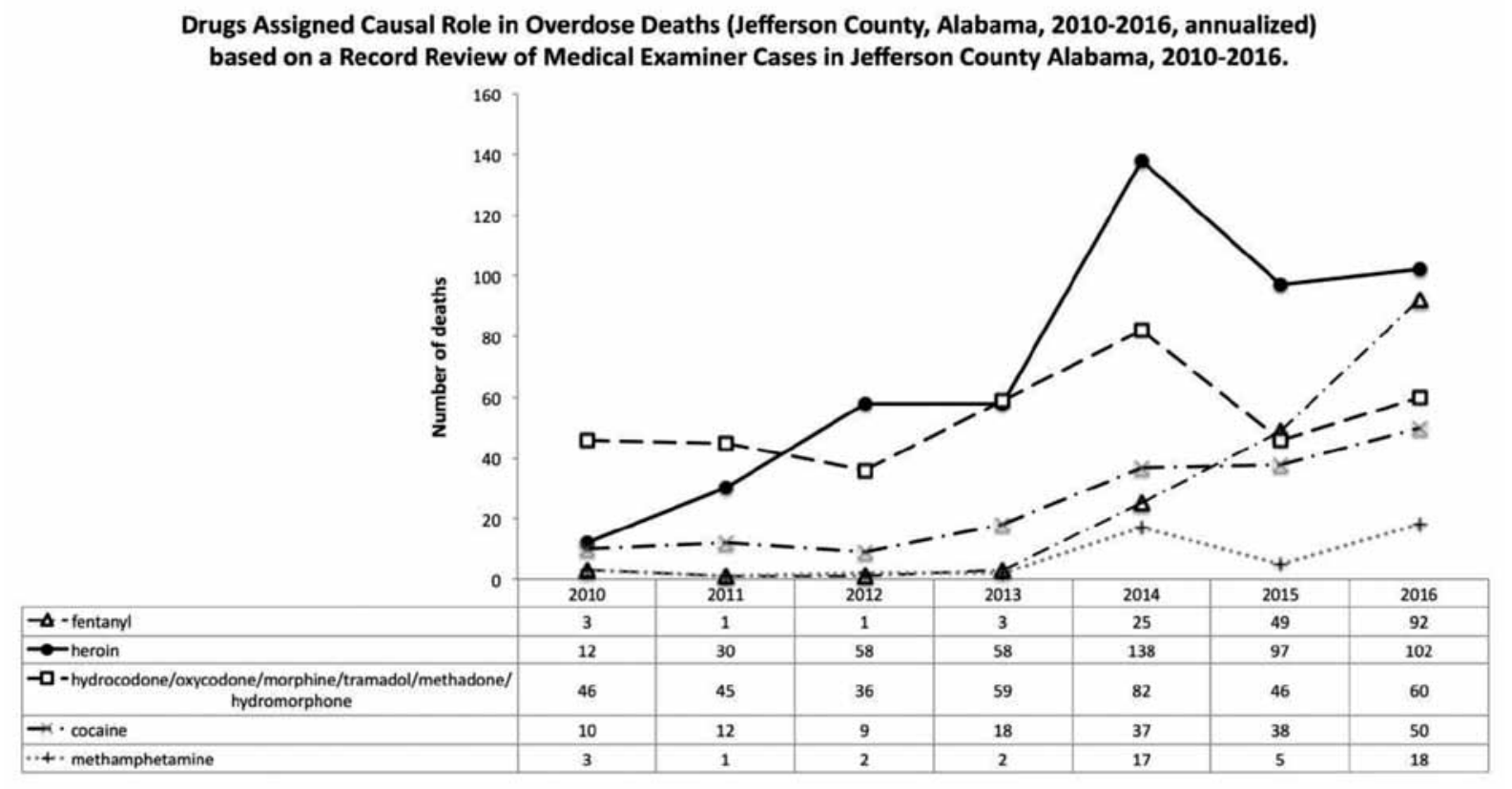

Fig. 7. Drugs assigned a causal role in overdose deaths (Jefferson County, Alabama, 2010 - 2016, annualized) based on record review of medical examiner cases in Jefferson County Alabama, 2010 - 2016.

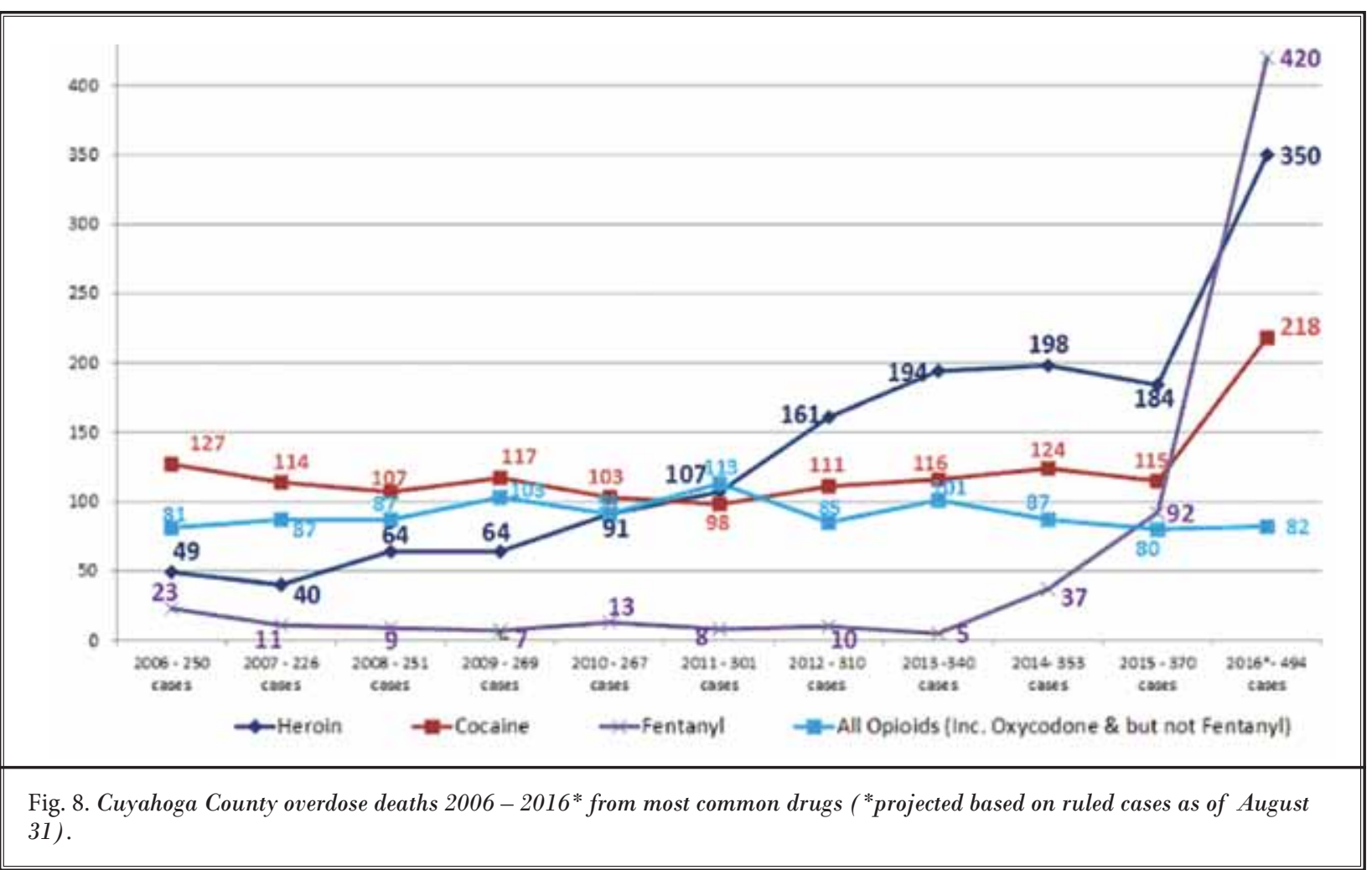


of natural and synthetic pain reliever contributed deaths, authors included prescription drugs in the same category with same intensity. These concepts are based on opioids being a significant risk factor for heroin use (37).

Redican et al (160) explored the etiologic factors and dynamics of prescription drug abuse in southwest Virginia. The extent of prescription drug abuse was high and the demographics of prescription drug users evolved into younger and men. They showed that over one half of methadone maintained consumers reported that they had abused benzodiazepines, along with opioids, and oxycodone, hydrocodone, methadone, and morphine were the most commonly used drugs prior to enrollment in the clinics. Further data highlighted the key etiologic factors in prescription drug abuse as persons with work-related injuries turning to abuse, wanting to get high, overprescribing and physician issues, lack of information, and cultural acceptance of drug taking as a problem-solving behavior. They clearly identified that the 2 most common sources for the abused prescription drugs were physicians and street dealers. Other factors included poverty, unemployment, and work-related injuries, and lack of public health education to learn the dangers of prescription opiate misuse and abuse.

Boscarino et al (161) completed phone interviews with a random sample of 705 chronic pain patients receiving opioid treatment in primary care and specialty pain treatment. They found that $26 \%$ of those reported a current opioid use disorder and $36 \%$ had a life-time opioid use disorder using the Diagnostic and Statistical Manual of Mental Disorders (DSM-V) criteria.

In a primary care sample receiving daily opioid therapy (102), results showed a frequency 4 times higher for opioid use disorders in the patients receiving opioid therapy compared with general population samples $(3.8 \%$ versus $0.9 \%)$. A study evaluating the risks in commercial and Medicaid insurance plans (101) showed an estimated possible misuse of $20 \%$ to $24 \%$.

Multiple investigators also have studied the issue of illicit drug use in chronic pain patients receiving controlled substances with variable prevalence rates (91,101,161-177). In contrast, Fishbain et al (178), in contradiction to present evidence, in a structured evidence-based review, concluded that chronic opioid therapy exposure will lead to abuse and addiction only in a small percentage of chronic pain patients, but a large percentage will demonstrate aberrant drugrelated behaviors and illicit drug use.

\subsection{Effectiveness of Opioid Therapy in Chronic Pain: Key Question 4. What is the EVIDENCE FOR THE THERAPEUTIC EFFICACY OF OPIOIDS IN MANAGING CHRONIC NON-CANCER PAIN?}

The effectiveness of chronic opioid therapy has been assessed in multiple RCTs and numerous observational studies, comprehensive reviews, and systematic reviews. However, an overwhelming proportion of the literature limits these assessments to short-term, specifically with RCTs and systematic reviews.

The recently published CDC guidelines for prescribing opioids for chronic pain by Dowell et al (42) assessed the evidence for opioid therapy. The assessment included observational studies and RCTs with significant limitations, characterized as low quality. They were unable to perform meta-analysis due to the limited number of studies, variability in study designs and clinical heterogeneity, and methodological shortcomings of the studies. None of the studies evaluated long-term benefits of opioids over one year for chronic pain. Apart from the lack of long-term assessments, opioids were associated with increased risks, including opioid use disorder, overdose, and death, with dose dependent effects. The CDC conducted a contextual evidence review to assist in developing the recommendations by providing an assessment of the balance of benefits and harms, values and preferences, and cost, consistent with the GRADE approach (42). Assessment of the effectiveness of opioid therapy in preparation for ASIPP guidelines in $2012(11,12)$ showed similar results as the CDC and other guidelines on the update of the guidelines.

Abdel Shaheed et al (179) performed a systematic review and meta-analysis assessing the efficacy, tolerability, and dose-dependent effects of opioid analgesics for low back pain. They included 20 RCTs of opioid analgesics with a total of 7,295 participants, with 13 trials (3,419 participants) assessing short-term effects on chronic low back pain. Among these, 19 opioid analgesic trials assessed patients with chronic low back pain, whereas one trial evaluated participants with subacute low back pain. In this assessment, 17 RCTs compared an opioid analgesic with placebo and 3 trials compared 2 opioid analgesics (180-199). However, most of the trials evaluated short-term use or outcomes. The maximum treatment period in all the studies was 12 weeks. The trials included oral hydromorphone (183), oxymorphone $(180,184,185)$, morphine $(181,186)$, tramadol $(182,187-192)$, tapentadol (193), oxycodone (193-196), transdermal buprenorphine $(194,197,198)$, transdermal fentanyl (181), and hydrocodone (199). In contrast to 
previous assessments, this systematic review rated the trials typically of high quality even though 17 of the 20 trials reported industry funding. The systematic review also showed that in half of the 13 trials evaluating short-term effects of chronic low back pain at least $50 \%$ of the participants withdrew owing to adverse events or lack of efficacy.

The results of this systematic review showed moderate quality evidence from 13 studies of chronic low back pain (3,419 participants) of an effect of single ingredient opioid analgesics on pain in the short-term. They also showed that there is high quality evidence from 6 studies (2,500 participants) that single-ingredient opioid analgesics relieved pain in the intermediate term. Combination opioid analgesics with acetaminophen or another simple analgesic showed a moderate evidence of pain relief in the intermediate term. Clinically important pain relief was not observed within the dose range evaluated, ranging from 40 to 240 MME per day. There was no significant effect of enrichment study design. However, in reference to functional status or disability outcomes $(186,191,192)$, there was no clinically significant reduction in disability for the short-term with either tramadol or morphine. Comparative assessment trials showed that there was a significant difference in treatment outcome between different strengths of transdermal buprenorphine for the short and intermediate term, with the $20 \mu \mathrm{g}$ per hour patch providing greater pain relief than the $5 \mu \mathrm{g}$ patch $(194,197,198)$. Similarly, oral oxycodone also provided greater pain relief with $40 \mathrm{mg}$ per day compared to transdermal buprenorphine $5 \mathrm{mcg}$ per hour for both short-term and intermediate-term relief.

In summary, in this assessment of chronic low back pain, opioid analgesics provided modest short-term pain relief, even though based on the conclusions, the effect is not likely to be clinically important within guideline recommended doses (Table 9). Further, evidence on long-term efficacy is lacking and the efficacy of opioid analgesics in acute low back pain is unknown.

Deyo et al (3) studied the role of opioids in acute low back pain and chronic low back pain. Opioid prescriptions for acute low back pain may inadvertently lead to long-term use, specifically if patients are provided with a large supply or simply continue to refill the prescriptions. They may also lead to drug dependence and long-term use (200). In this review, the authors opined that most RCTs of opioids for back pain have assessed chronic low back pain, but none of the trials lasted beyond 4 months. They also showed that all the trials had high dropout rates (more than 20\%), mostly because of adverse effects or inefficacy and the evidence was meager and inconclusive. They reviewed the same studies utilized in other reviews and concluded that of 15 RCTs $(183-191,193,195,198,201-203)$ in the systematic review by Chaparro et al (204), 5 studies (187-191) examined the weak opioid agonist tramadol, 2 studies $(198,202)$ assessed transdermal buprenorphine, and 7 RCTs (183$186,193,195,203)$ compared long-acting strong opioids. Deyo et al (3) concluded that there was no evidence that opioids improved return to work or reduced the use of other treatments. Ironically, opioids may limit the effectiveness of other treatments.

Chaparro et al (204) compared opioids with placebo or other treatments for chronic low back pain in an updated Cochrane review. In this assessment, they included 15 trials with 5,540 participants (183$191,193,195,198,201-203)$. The results showed that tramadol, examined in 5 trials (187-191) with 1,378 participants, was found to be better than placebo for pain (low quality evidence) and function (moderate quality evidence). Meta-analysis was performed as shown in Fig. 9. Transdermal buprenorphine, examined in 2 trials with 653 participants $(198,202)$, showed some difference for pain (very low quality evidence), with no difference compared to placebo for function (very low quality evidence). Strong opioids (morphine, hydromorphone, oxycodone, oxymorphone, and tapentadol), examined in 7 trials $(183-186,193,195,203)$ with involvement of 1,887 participants, were better than placebo for pain (moderate quality evidence) and function (moderate quality evidence). Meta-analysis was performed for strong opioids as shown in Fig. 10. They concluded that there is some evidence (very low to moderate quality) for the short-term efficacy for both pain and function of opioids to treat chronic low back pain compared to placebo. They also noted that the few trials that compared opioids to NSAIDs or antidepressants did not show any difference regarding pain and function.

The Canadian Agency for Drugs and Technologies in Health has provided a review of the clinical efficacy and safety of long-acting opioids for chronic non-cancer pain (205). In this systematic review, the authors assessed the use of long-term opioids in chronic pain. They concluded that there was insufficient evidence for assessing long-acting opioids, and insufficient evidence to discriminate between the 4 long-acting opioids (morphine, hydromorphone, oxycodone, and fentanyl) in terms of efficacy and safety. 
Responsible, Safe, and Effective Prescription of Opioids for Chronic Non-Cancer Pain

\begin{tabular}{|c|c|c|c|c|c|c|c|c|c|c|c|c|c|}
\hline & 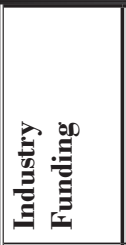 & 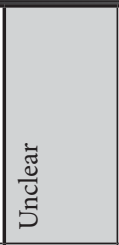 & $\cong$ & $\begin{array}{l}\tilde{\Xi} \\
\tilde{J} \\
5 \\
5\end{array}$ & 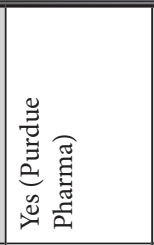 & 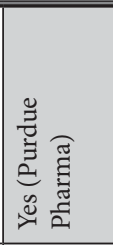 & 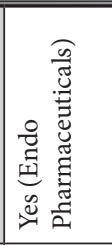 & 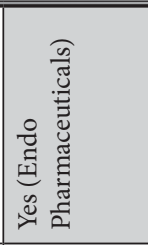 & 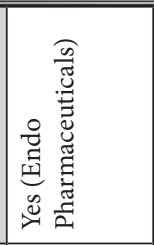 & 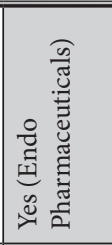 & 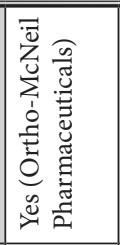 & 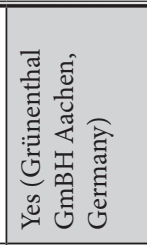 & 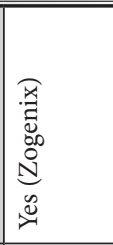 \\
\hline & 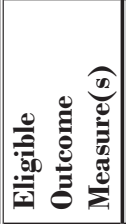 & $\underset{5}{5}$ & 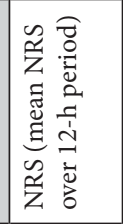 & 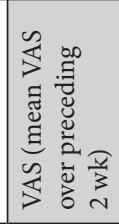 & 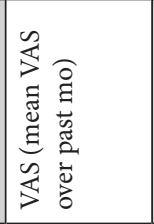 & 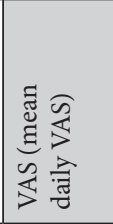 & 岇 & 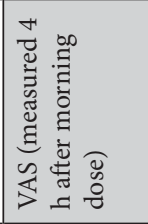 & 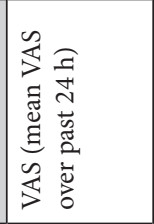 & $\underset{5}{5}$ & 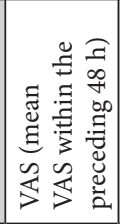 & $\sum_{i}^{\infty}$ & 㫕 \\
\hline & 章 & $\begin{array}{l}2 \\
-4 \\
3 \\
3 \\
-3\end{array}$ & 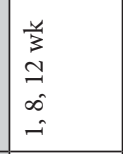 & g & $\begin{array}{l}\infty \\
\text { घै } \\
\text { उँ }\end{array}$ & $\begin{array}{l}\frac{4}{3} \\
\infty\end{array}$ & 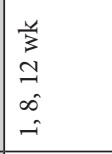 & 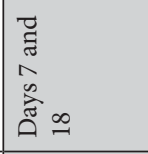 & 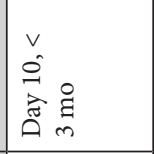 & 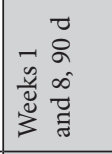 & $\overrightarrow{\vec{\sigma}}$ & 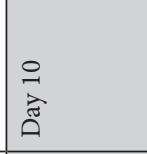 & 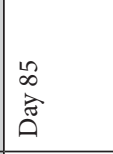 \\
\hline & 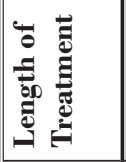 & $\begin{array}{l}\stackrel{̊}{\Xi} \\
\\
\end{array}$ & $\begin{array}{l}\frac{u}{3} \\
\beth \\
I\end{array}$ & $\stackrel{̊}{\Xi}$ & $\begin{array}{l}\frac{4}{3} \\
\infty\end{array}$ & $\begin{array}{l}\frac{y}{3} \\
\infty\end{array}$ & $\begin{array}{l}\text { un } \\
\text { I } \\
\underline{I}\end{array}$ & \begin{tabular}{|l}
$\vec{\nabla}$ \\
$\stackrel{\sim}{-}$
\end{tabular} & 思 & 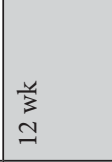 & $\begin{array}{l}\vec{\sigma} \\
\vec{a}\end{array}$ & $\stackrel{\Xi}{0}$ & 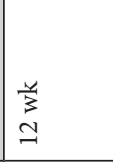 \\
\hline & 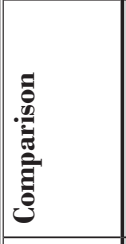 & 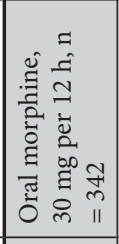 & 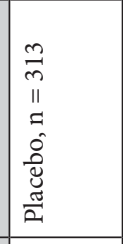 & 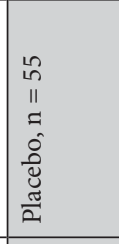 & 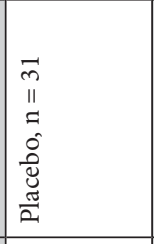 & 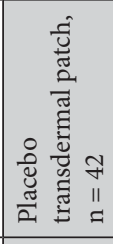 & 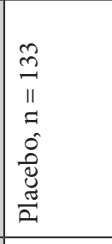 & 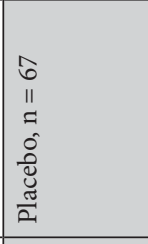 & 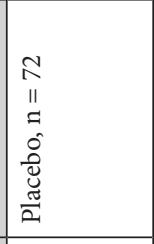 & 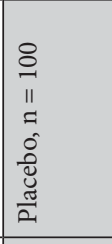 & 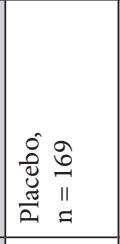 & 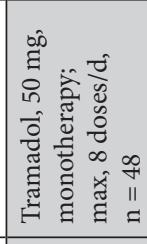 & 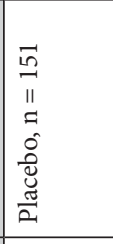 \\
\hline & 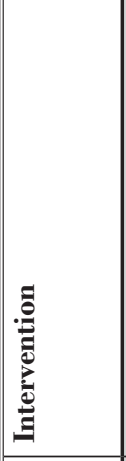 & 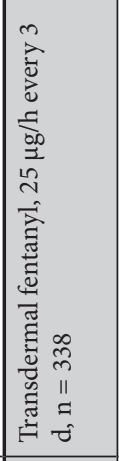 & 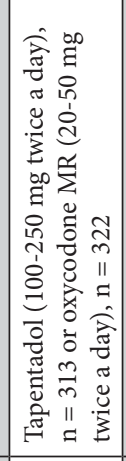 & 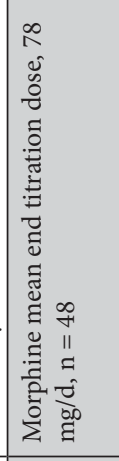 & 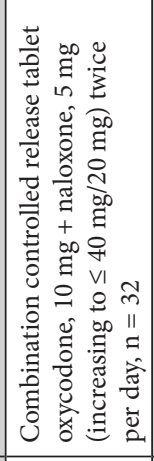 & 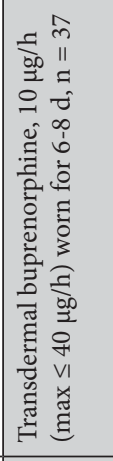 & 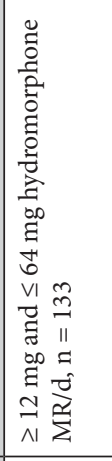 & 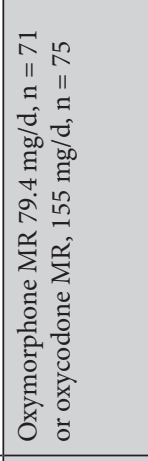 & 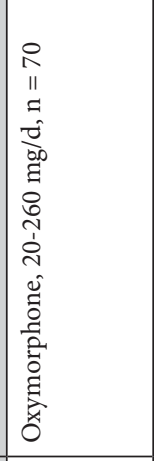 & 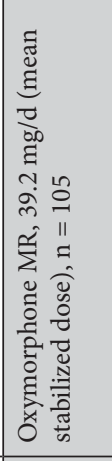 & 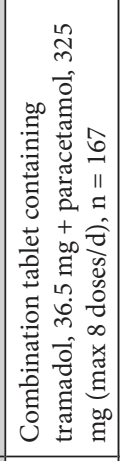 & 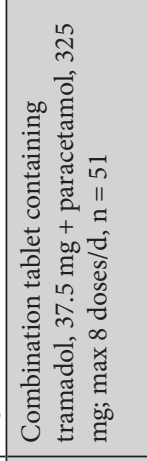 & 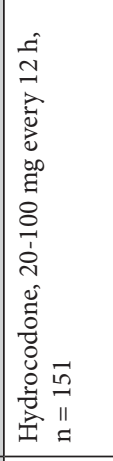 \\
\hline & 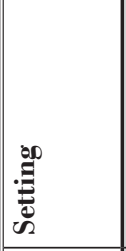 & 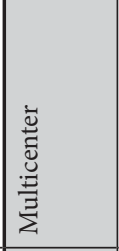 & 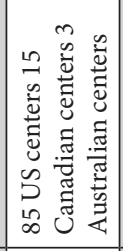 & 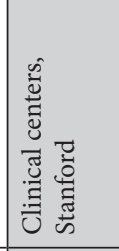 & 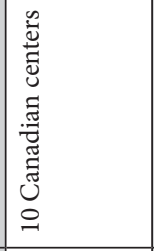 & 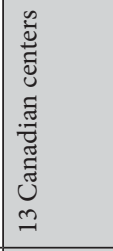 & 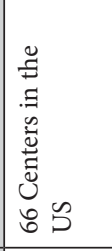 & 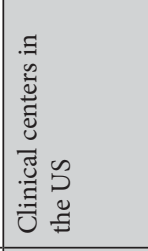 & 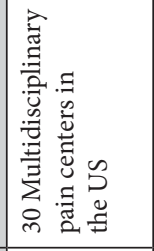 & 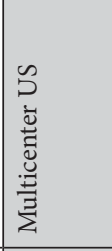 & s & 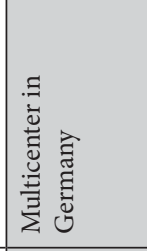 & 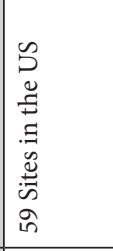 \\
\hline & 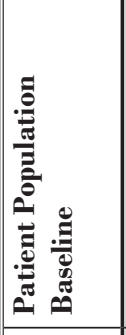 & 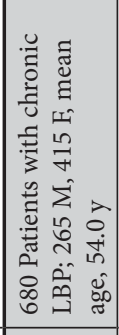 & 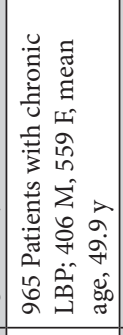 & 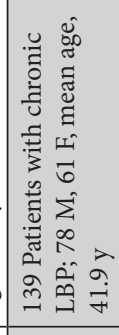 & 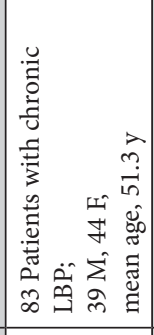 & 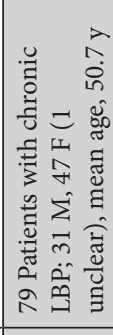 & 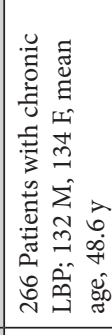 & 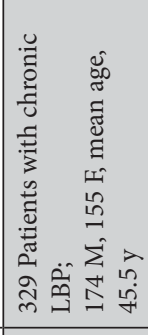 & 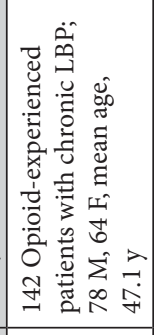 & 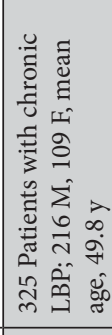 & 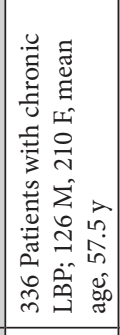 & 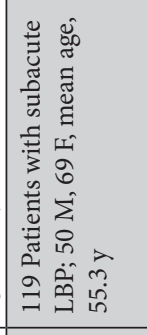 & 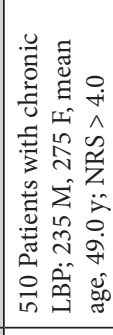 \\
\hline & 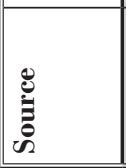 & 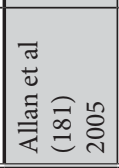 & 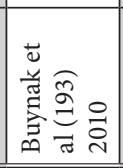 & 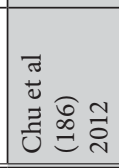 & 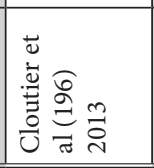 & 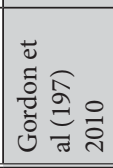 & 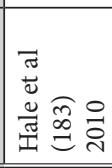 & 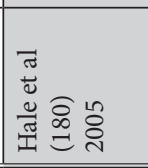 & 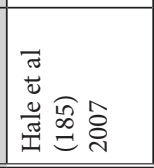 & 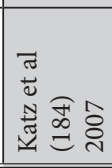 & 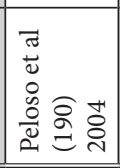 & 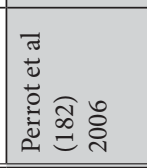 & 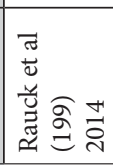 \\
\hline
\end{tabular}


Pain Physician: Opioid Special Issue 2017; 20:S3-S92

\begin{tabular}{|c|c|c|c|c|c|c|c|c|c|}
\hline 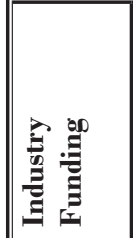 & 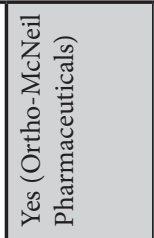 & 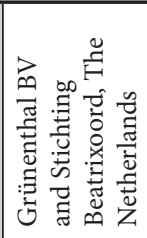 & 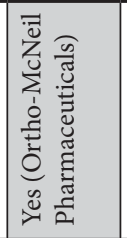 & 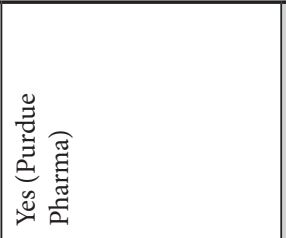 & 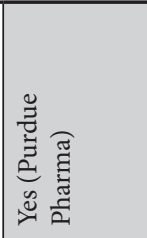 & 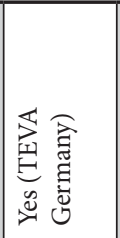 & 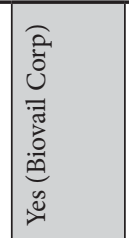 & \begin{tabular}{|l}
$\overrightarrow{\tilde{J}}$ \\
$\tilde{u}$ \\
$\tilde{J}$
\end{tabular} & $\begin{array}{l}\bar{J} \\
5 \\
5 \\
5 \\
\text { n }\end{array}$ \\
\hline 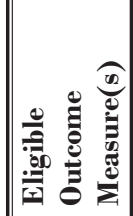 & 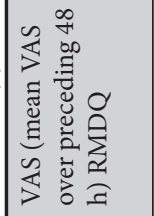 & 齐 & 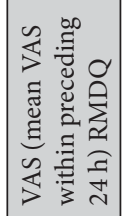 & 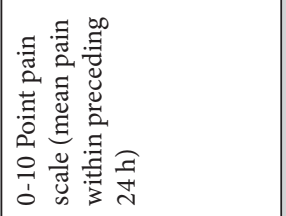 & 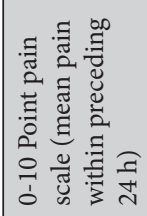 & 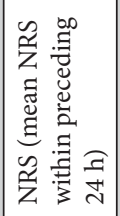 & 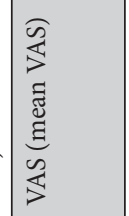 & 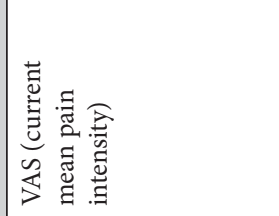 & 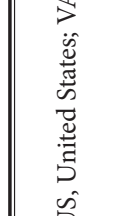 \\
\hline 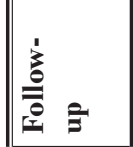 & $\begin{array}{l}\vec{\sigma} \\
\bar{\sigma}\end{array}$ & $\begin{array}{l}\vec{\theta} \\
\pm\end{array}$ & $\vec{F}$ & 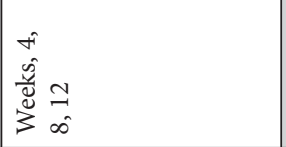 & $\begin{array}{l}\frac{4}{3} \\
\infty\end{array}$ & $\begin{array}{l}\frac{y}{3} \\
+\end{array}$ & 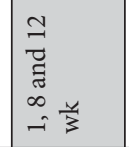 & 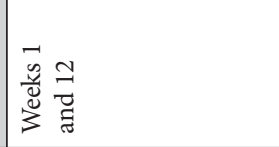 & 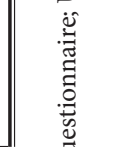 \\
\hline 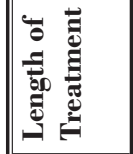 & $\begin{array}{l}\vec{\sigma} \\
\vec{a}\end{array}$ & $\begin{array}{l}\vec{t} \\
\pm \\
\end{array}$ & $\begin{array}{l}\frac{4}{3} \\
+\end{array}$ & $\begin{array}{l}\text { y. } \\
\text { In }\end{array}$ & $\begin{array}{l}\overrightarrow{0} \\
\text { வ }\end{array}$ & 豙 & 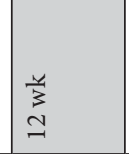 & $\begin{array}{l}\frac{y}{3} \\
\text { I }\end{array}$ & 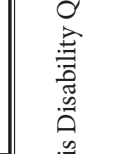 \\
\hline 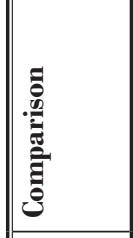 & 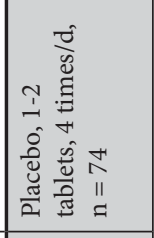 & 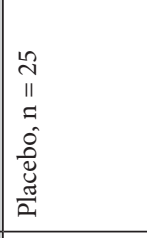 & 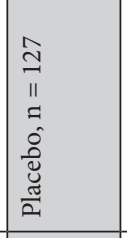 & 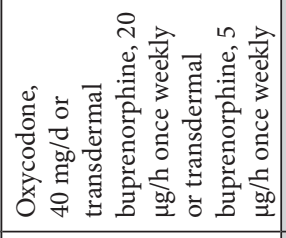 & 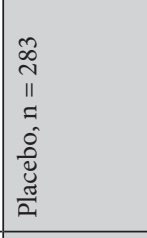 & 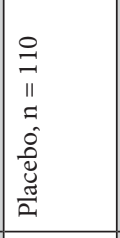 & 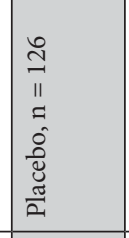 & 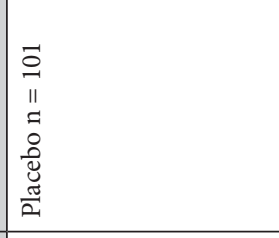 & 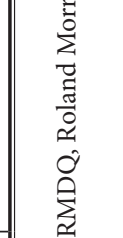 \\
\hline $\mid$ & 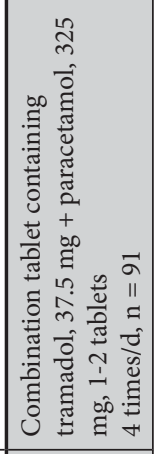 & 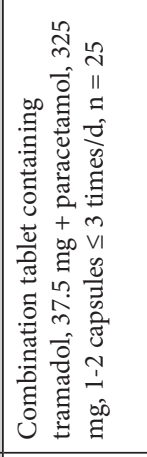 & 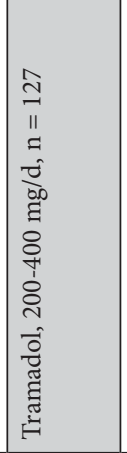 & 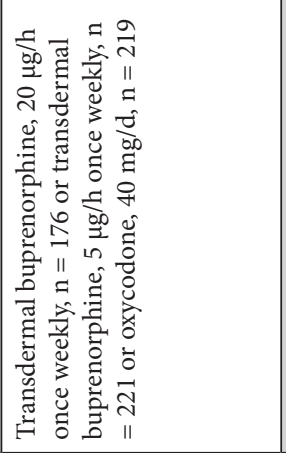 & 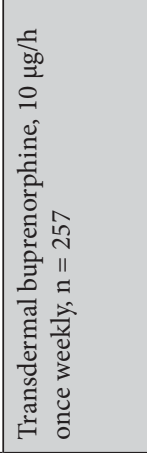 & 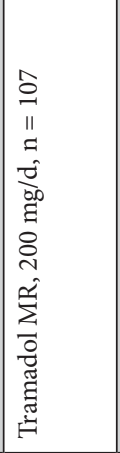 & 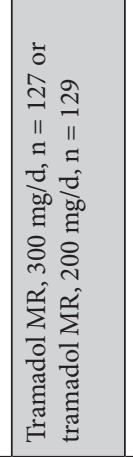 & 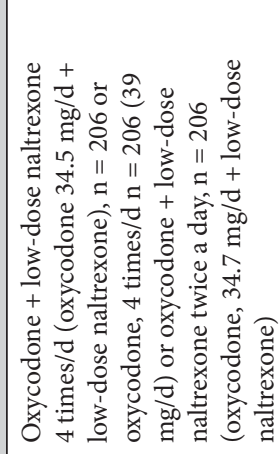 & 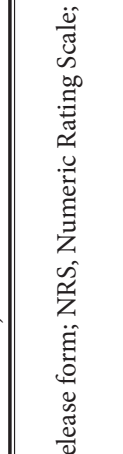 \\
\hline 告 & 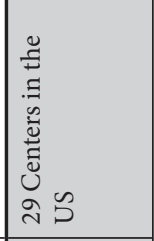 & 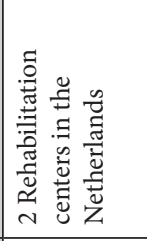 & 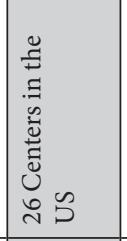 & 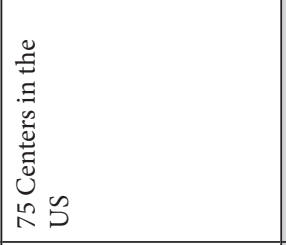 & 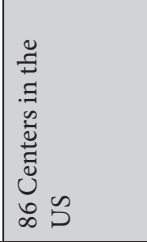 & 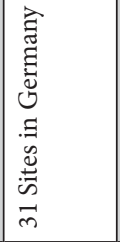 & 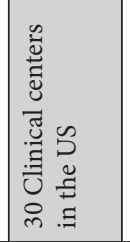 & 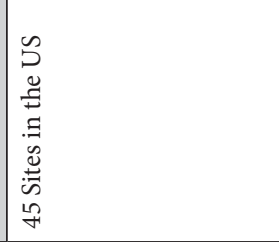 & 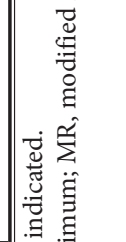 \\
\hline 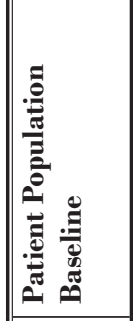 & 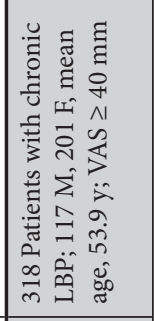 & 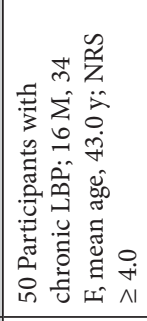 & 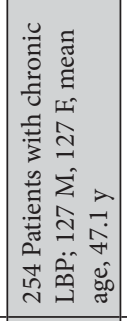 & 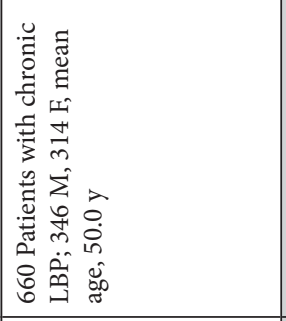 & 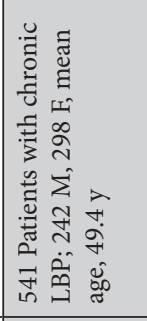 & 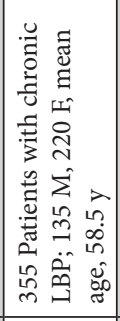 & 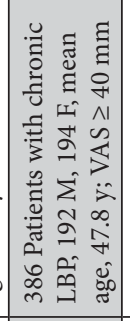 & 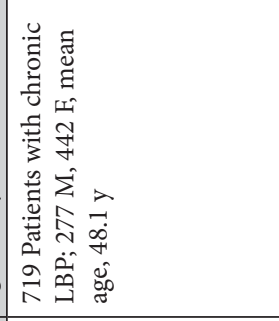 & 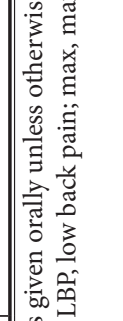 \\
\hline 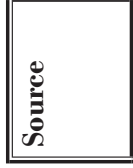 & 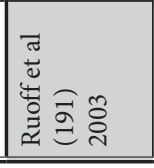 & 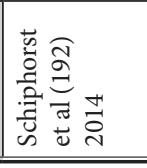 & 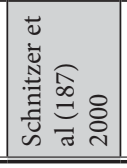 & 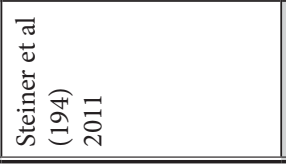 & 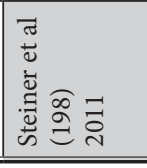 & 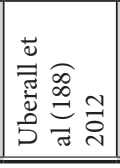 & 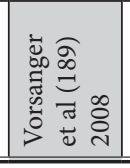 & 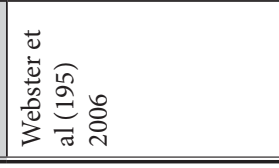 & 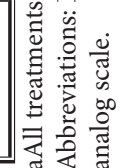 \\
\hline
\end{tabular}


Responsible, Safe, and Effective Prescription of Opioids for Chronic Non-Cancer Pain

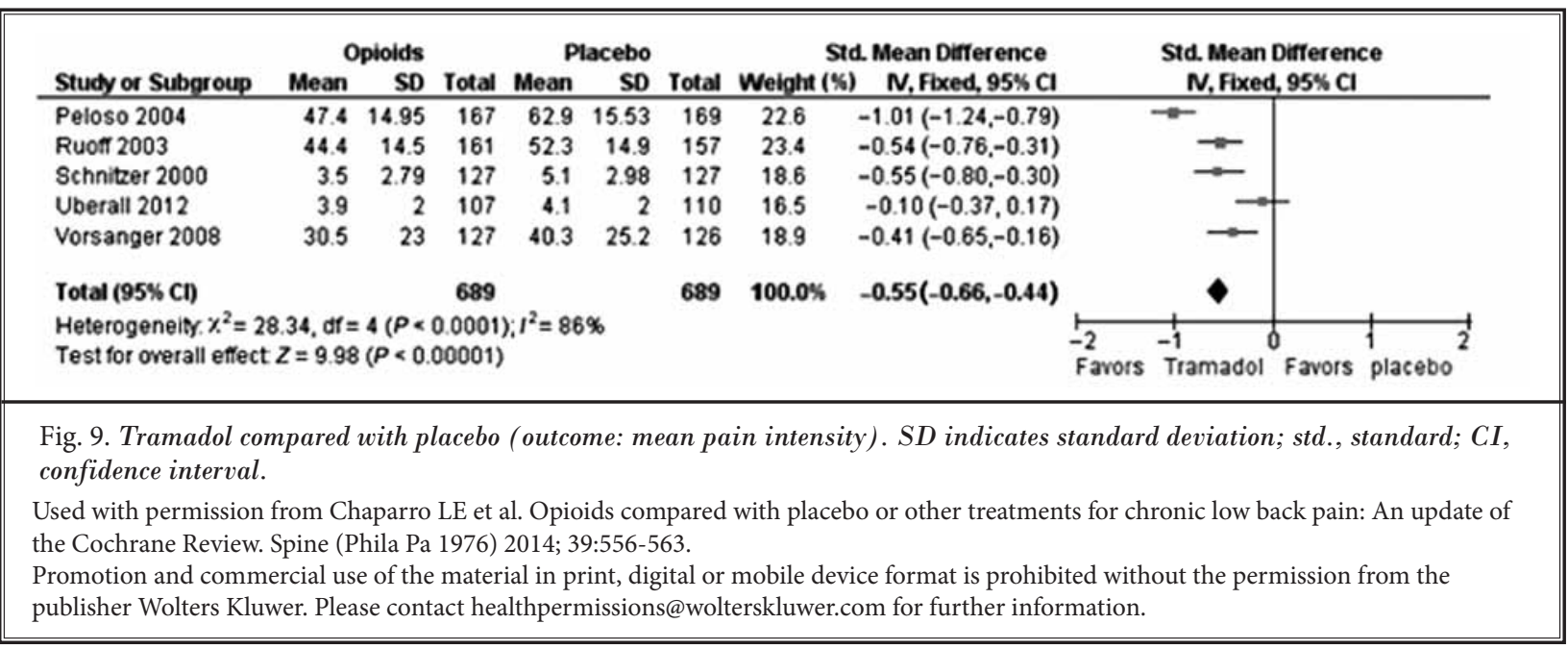

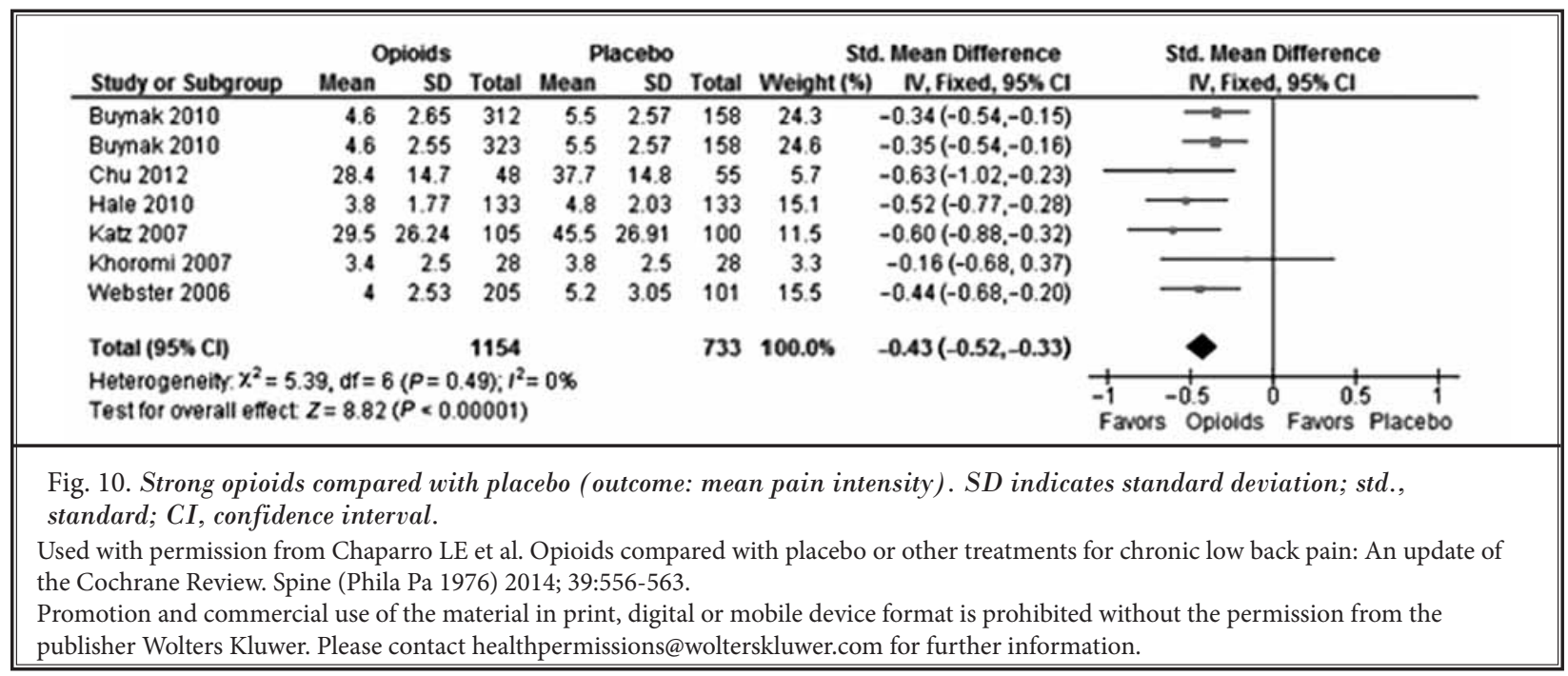

Chou et al (206) attempted to assess the effectiveness of long-term opioid therapy for chronic pain in a systematic review. They were unable to find any studies of opioid therapy versus no opioid therapy evaluating long-term outcomes of one year related to pain, function, QOL, opioid abuse, or addiction. There was insufficient evidence to determine the effectiveness of long-term opioid therapy for improving chronic pain and function. However, evidence supported a dose dependent risk for serious harms.

Chung et al (207), in a systematic review and metaanalysis, assessed drug therapy for the treatment of chronic nonspecific low back pain. Their follow-up period in this trial ranged from 4 to 24 weeks. Among the included studies, 8 of them included nonopioids and 4 of them included antidepressants, and there were only 3 studies (189-191) of tramadol versus placebo with 613 patients and 4 studies $(184,185,193,195)$ of opioids versus placebo with 1,302 patients. The studies of mild opioids showed results in favor of opioids for pain and function at 12-week follow-up. The studies of oxycodone, oxymorphone, and buprenorphine showed favorable results at 12-week follow-up.

Reinecke et al (208) performed a meta-analysis of the analgesic efficacy of opioids in chronic pain and compared strong opioids, weak opioids, nonopioids, psychotherapy, and physiotherapy. They opined that previous meta-analysis and RCTs lacked methodological homogeneity and comparable data. Consequently, they analyzed the maximum analgesic efficacies of opioids and nonopioids compared with placebo, and of physiotherapy and psychotherapy compared with active 
or waiting-list controls. With inclusion of 46 studies, they showed most pain reduction on a 100 point scale, of 12.0 for strong opioids, followed by 10.6 for weak opioids, 8.4 for nonopioids, 5.5 for psychotherapy, and 4.5 for physiotherapy. They also showed high drop-out rates in the pharmacological studies. The limitation of this metaanalysis was limited to adjusted indirect comparisons, due to lack of enough eligible head-to-head trials. Further, heterogeneity of the pre/post differences in control groups did not follow the definition of a common comparator. They concluded that even though there were statistically significant differences between maximum treatment efficacies, no intervention per se produced clinically important improvement in average pain intensity. Thus, they concluded that opioids alone were inappropriate and multimodal treatment programs may be required.

Welsch et al (209), in a systematic review and metaanalysis, also compared the efficacy, tolerability, and safety of chronic non-cancer pain treatment with opioids and nonopioid analgesics in randomized headto-head comparisons. They included 10 RCTs with 3,046 participants with study durations ranging from 4 to 12 weeks. In this meta-analysis, 5 studies compared tramadol with NSAIDs in osteoarthritis pain and one trial compared tramadol to flupirtine in low back pain, morphine was compared to antidepressants in 2 studies, and an anticonvulsant and an antiarrhythmic were studied in different neuropathic pain syndromes with one study for each. The results showed that patients dropped out due to adverse events more frequently with opioids than nonopioid analgesics. There were no significant differences between opioids and nonopioids in reference to adverse events or drop-out rates due to lack of efficacy; however, they concluded that nonopioid analgesics were superior to opioids in terms of improvement of physical function and tolerability in short-term therapy of 4 to 12 weeks for neuropathy, low back, and osteoarthritis pain.

Gaskell et al (210), in a Cochrane Database systematic review, assessed oxycodone for neuropathic pain and fibromyalgia in adults with inclusion of 3 studies including 204 painful diabetic neuropathy patients and 50 postherpetic neuralgia patients. None of the studies reported the proportion of participants experiencing at least $50 \%$ pain relief or who were very much improved, even though one study reported the proportion with at least $30 \%$ pain relief, 2 reported at least moderate pain relief, and one reported the number of participants who considered treatment to be moderately effective. However, the authors concluded that there was no convincing, unbiased evidence suggesting that oxycodone in long-acting form is of value in treating people with painful diabetic neuropathy or postherpetic neuralgia.

In a comparative effectiveness review of noninvasive treatments for low back pain, Chou et al (211) updated their previous reviews of American Pain Society and American College of Physicians reviews which were conducted through October 2008. In this assessment, they concluded that for chronic low back pain, NSAIDs and tramadol were associated with moderate effects on pain versus placebo, and opioids, duloxetine, and benzodiazepines were associated with small effects. They also showed that effects on function were small for NSAIDs, opioids, tramadol, and duloxetine. Further, in their analysis, head-to-head comparisons were limited, but showed no clear difference between different NSAIDs, different long-acting opioids, or long-acting versus short-acting opioids. In addition, the evidence was too inconsistent to determine the effects of opioids versus NSAIDS.

Santos et al (212) performed a Cochrane review to assess tapentadol for chronic musculoskeletal pain in adults. They included 4 parallel-design RCTs of moderate quality including 4,094 participants with osteoarthritis or back pain or both. The authors concluded that tapentadol extended-release was associated with a reduction in pain intensity in comparison to placebo and oxycodone; however, they also added that the clinical significance of the results is uncertain due to the modest difference between interventions and efficacy outcomes, high heterogeneity in some comparisons and outcomes, high withdrawal rates, and lack of data for the primary outcome in some studies. Overall, tapentadol was associated with a more favorable safety profile and tolerability than oxycodone.

Cepeda et al (213), in 2007, published the results of a systematic review and meta-analysis of tramadol for osteoarthritis. This systematic review included 11 RCTs (214-224), concluding that patients who receive tramadol reported less pain associated with a higher degree of global improvement. They reported that pain relief also improved the function in patients with osteoarthritis, even though these benefits were small.

Sandoval (225), in 2000, published a systematic literature review of the reasons for administration prescription patterns, effectiveness, and side effects of oral methadone, which included 21 studies (226244): one small RCT (244), 13 case reports (226-236), and 7 case series (237-243) involving 545 patients 
with heterogenous non-cancer pain conditions. The results of this review showed meaningful outcomes in $59 \%$ of the patients with a statistically significant improvement in pain with methadone at $20 \mathrm{mg}$ per day compared to the placebo based on one RCT. This review based on the author's analysis and our analysis must be reported with caution based on the side effect profile of methadone including deaths and the paucity of the literature.

McNicol et al (245) assessed opioids for neuropathic pain in a Cochrane review. This was an updated version of a manuscript published in 2006 (246), which included 23 trials. The updated version included 31 trials $(203,244,247-$ 276) studying 10 different opioids. This included 23 studies from the original 2006 review and 8 additional studies from this updated review. Of these, only 14 studies, with involvement of 845 participants, were of intermediate duration lasting 12 weeks or less and the other studies were less than 6 weeks. The authors concluded that shortterm studies provide only equivocal evidence regarding the efficacy of opioids in reducing the intensity of neuropathic pain, whereas intermediate studies demonstrated significant efficacy of opioids over placebo, even though these results were likely to be subject to significant bias because of the small size, short duration, and potentially inadequate handling of dropouts.

van Ojik et al (277) reviewed evidence-based choice of strong-acting opioids in the elderly in treatment of chronic cancer and non-cancer pain, utilized studies from 1966 to 2011 and a set of 23 validated criteria. They found that there was a lack of available studies for the use of hydromorphone, methadone, and oxycodone in the treatment of chronic malignant or nonmalignant pain in the frail elderly. Overall they showed that there is little or no evidence for the effectiveness of opioids in the treatment of chronic pain in the frail elderly. Among the included studies, Griessinger et al (278), in an open label, uncontrolled, observational study, concluded that transdermal buprenorphine was effective and well tolerated in the treatment of chronic cancer and non-cancer pain, irrespective of the patient's age. However, this study also showed the initial dose of buprenorphine was $35,52.5$, or $70 \mathrm{mcg}$ per hour in $78 \%, 16 \%$, and $5 \%$ of the patients, respectively. Further, during buprenorphine therapy, almost $50 \%$ of the patients required concomitant analgesic therapy with either NSAIDs or opioids. In another study, Gianni et al (279) also assessed the safety of transdermal buprenorphine in 93 elderly patients with chronic nonmalignant pain. The buprenorphine dose in this study was 75 mcg per hour initially and it was between 11.7 and 70 mcg per hour with a mean dose of $34.2 \mathrm{mcg}$ per hour at the end of 3 months. The authors of this open label uncontrolled observational study also concluded that buprenorphine was effective and safe in the treatment of elderly patients.

Krashin et al (280), in a review of the role of opioids in the management of HIV-related pain, concluded that there was undertreatment and increased complexity of management of patients with HIV, higher risks of side effects, higher rates of comorbid psychiatric illness and substance abuse, complex anti-retroviral drug regimens, and increased tolerance. In general, patients with HIV-related pain required high doses of opioids. There are no studies evaluating the effectiveness of individual drugs and their efficacy and adverse effect profile in HIV-related pain.

Over the years, multiple studies have been published describing the role of opioid therapy and disability (73,91,281-292). A 2006 Danish study by Erikson et al (281) showed that opioid use for chronic pain was significantly associated with reporting of severe pain, poor self-rated health, unemployment, higher health care use, and lower self-rated QOL. In a study in Denmark by Breivik et al (73), the results showed that liberal prescriptions of opioids for chronic pain was associated with worse pain, increased health care utilization, and reduced activity levels. Similar to the above reports, others $(282,283)$ have reported increased functional disability among chronic pain patients receiving longterm opioid treatment (282) and reduced functional outcomes after therapy $(283,284)$. Kidner et al $(285)$ also showed higher pain intensity, greater disability, and higher levels of depression in patients with chronic musculoskeletal pain on opioid therapy compared to those without opioid therapy in 2009. Franklin et al $(286,287)$, in 2 studies of work-related back injuries, showed that patients receiving opioids for more than 7 days were twice as likely to remain work disabled at one year (286) and long-term use increased dosages substantially without significant improvement in functional status (287). Webster et al (288), in the United States, showed an increased association of longer disability with an increasing opioid dosage. Ashworth et al (289), in the United Kingdom, showed patients receiving opioids had worse pain, functioning, self efficacy, catastrophizing fear of movement, and depression. Similarly, a Canadian study (290) by Cross et al showed continued disability in a broader range of painful musculoskeletal conditions. Sites et al (291), describing increases in the use of prescription opioid analgesics, 
also showed lack of improvement in disability. In addition, long-term disability and relation to poor response to surgical intervention in the cervical and lumbar spine also have been reported $(291,292)$; whereas patients with lumbar disc herniation showed an increased use of opioids at 4-year follow-up (284). However, there are also conflicting reports about the influence of pain intensity on both disability $(293,294)$ and opioid use (295-297). Ashworth et al (289) also showed that after adjusting for a substantial number of potential confounders, opioids were associated with slightly worse functioning in back pain patients at 6-month follow-up. However, in lumbar disc herniation, Radcliff et al (284) showed that at 4-year follow-up, there were no significant differences in primary or secondary outcome measures or treatment effect of surgery between opioid and nonopioid medication patients. Further, assessment of physical function and opioid use in patients with neuropathic pain also showed lack of improvement of physical functioning and disability in patients with neuropathic pain receiving opioids compared with those who were not prescribed, even after adjusting for disease severity. Essentially, patients prescribed opioid therapy on an ongoing basis showed higher disability and lower physical functioning scores.

In contrast to the scientific studies, a patient focused survey (34) by the Washington Post-Kaiser Family Foundation of long-term opioid therapy revealed some surprising results: $92 \%$ of patients attested to the fact that prescription opioids reduced their pain at least somewhat well and $53 \%$ attested that prescription opioids reduced their pain very well. In addition, $57 \%$ stated that their QOL was better than if they had not taken the medications. However, in-depth evaluation of the data analysis shows that even though a small proportion of patients reported improvement in physical health or mental health, the majority of the patients $(70 \%)$ on long-term opioid usage were disabled, handicapped, or suffered with a chronic disease which kept them from participating fully in work, school, housework, or other activities. In addition, $20 \%$ reported a positive impact on their mental health and $15 \%$ reported a positive effect on the personal relationships. Consequently, the survey shows a population not compatible to the general population, yielding different results than the scientific studies. The results of this survey also raise further questions into the present concepts of returning to work as an important feature of improvement in physical health which is not feasible in $70 \%$ of the population on opioid therapy $(33,34)$.

\subsection{Adverse Consequences of Opioid Therapy: Key Question 5. What are the ADVERSE CONSEQUENCES/HARMS OF OPIOID THERAPY?}

The risks of prescribing opioids range from mild side effects to catastrophic complications. The major adverse consequences of opioids include tolerance, physical dependence, addiction, and death. However, the majority of the patients treated with opioids experienced gastrointestinal or central nervous system-related adverse events, the most common of which includes constipation, nausea, and somnolence. This often leads to discontinuation of opioid therapy (298). In a systematic review of symptoms and side effects of opioid therapy in chronic non-cancer pain, Jonsson et al (299) showed that fatigue, cognitive dysfunction, dry mouth, sweating, and weight gain were the most frequently reported side effects. In addition, they also reported that these adverse effects were 8-fold higher than those reported voluntarily. In another meta-analysis of effectiveness and side effects by Furlan et al (300), constipation and nausea were shown to be clinically and statistically significant. Kalso et al (301) showed about $80 \%$ of patients experienced at least one adverse event, with constipation in $41 \%$ of the patients, nausea in $32 \%$ of the patients, and somnolence in $29 \%$ of the patients. Over the years, multiple authors have described a wide range of side effects $(55,94,301$ 303) including constipation, pruritus, respiratory depression, nausea, vomiting, delayed gastric emptying, sexual dysfunction, muscle rigidity and myoclonus, sleep disturbance, diminished psychomotor performance, cognitive impairment, hyperalgesia, dizziness, sedation, and multiple drug interactions with involvement of multiple systems. Among multiple adverse effects, other than those related to dependency, addiction, death, and treatment resistant depression, opioid-associated endocrinopathy with androgen deficiency has been described as a common problem (304-315). The syndrome is associated with inappropriately low levels of gonadotrophin (follicle stimulating hormone and luteinizing hormone), leading to inadequate production of sex hormones, particularly testosterone (307).

The role of opioid hyperalgesia has been described with extensive implications (303). The opioid-induced hyperalgesia $(\mathrm{OIH})$ is characterized by a paradoxical response whereby a patient receiving opioids for treatment of pain could actually become more sensitive to certain painful stimuli (303). OIH has been described as a distinct, definable, and characteristic phenomenon that could explain loss of opioid efficacy in some patients (303). OIH results from neuroplastic changes in 
the peripheral and central nervous system that lead to sensitization of pronociceptive pathways with multiple proposed mechanisms (303).

Further, an assessment of prescription long-acting opioids in mortality in patients by Ray et al (17) showed further disturbing news that apart from contributing to accidental overdoses, opioids may also contribute to cardiac-related deaths and other fatalities. Ray et al showed that unintentional overdoses accounted for about $18 \%$ of the deaths among opioid users, compared to $8 \%$ of other patients. They concluded that prescribing long-acting opioids for chronic non-cancer pain, compared with anticonvulsants and antidepressants, was associated with a significantly increased risk of all-cause mortality, including deaths from causes other than overdose with a modest absolute risk differ- ence. Of these, more than one-half were cardiovascular deaths. This increased risk, confined to the first 180 days of opioid therapy, was present for long-acting opioid doses of $60 \mathrm{mg}$ or less of morphine equivalents. Other important complications include opioid induced respiratory depression, specifically in patients with obstructive sleep apnea syndrome. The Canadian guidelines described the prevalence of various adverse effects of opioids as shown in Table 10 (314).

The CDC has reported escalating rates of deaths from opioid overdoses from 7.9 per 100,000 in 2013 to 9.0 per 100,000 in 2014 , a $14 \%$ increase just in one year demonstrating a continuous increase since 2000 of $300 \%$ (36). As shown in Fig. 11, opioids were involved in 28,647 deaths, or $61 \%$ of all drug overdose deaths, with 18,893 deaths due to prescription opioids $(36,315)$.

Table 10. Adverse effects of opioids.

\begin{tabular}{|l|c|c|c|c|}
\hline Adverse effect & $\begin{array}{c}\text { Number } \\
\text { of Studies }\end{array}$ & $\begin{array}{c}\text { Incidence in } \\
\text { Opioid Group }\end{array}$ & $\begin{array}{c}\text { Incidence in } \\
\text { Placebo Group }\end{array}$ & Difference (95\% CI) \\
\hline Nausea & 38 & $28 \%$ & $9 \%$ & $17 \%(13 \%$ to $21 \%) \mathrm{P}<0.00001$ \\
\hline Constipation & 37 & $26 \%$ & $7 \%$ & $20 \%(15 \%$ to $25 \%) \mathrm{P}<0.00001$ \\
\hline Somnolence/drowsiness & 30 & $24 \%$ & $7 \%$ & $14 \%(10 \%$ to $18 \%) \mathrm{P}<0.00001$ \\
\hline Dizziness/vertigo & 33 & $18 \%$ & $5 \%$ & $12 \%(9 \%$ to $16 \%) \mathrm{P}<0.00001$ \\
\hline Dry-skin/ itching/pruritus & 25 & $15 \%$ & $2 \%$ & $10 \%(5 \%$ to $15 \%) \mathrm{P}<0.0001$ \\
\hline Vomiting & 23 & $15 \%$ & $3 \%$ & $11 \%(7 \%$ to $16 \%) \mathrm{P}<0.00001$ \\
\hline
\end{tabular}

Source: Canadian Guideline for Safe and Effective Use of Opioids for Chronic Non-Cancer Pain@ 2010 National Opioid Use Guideline Group (NOUGG) (314).

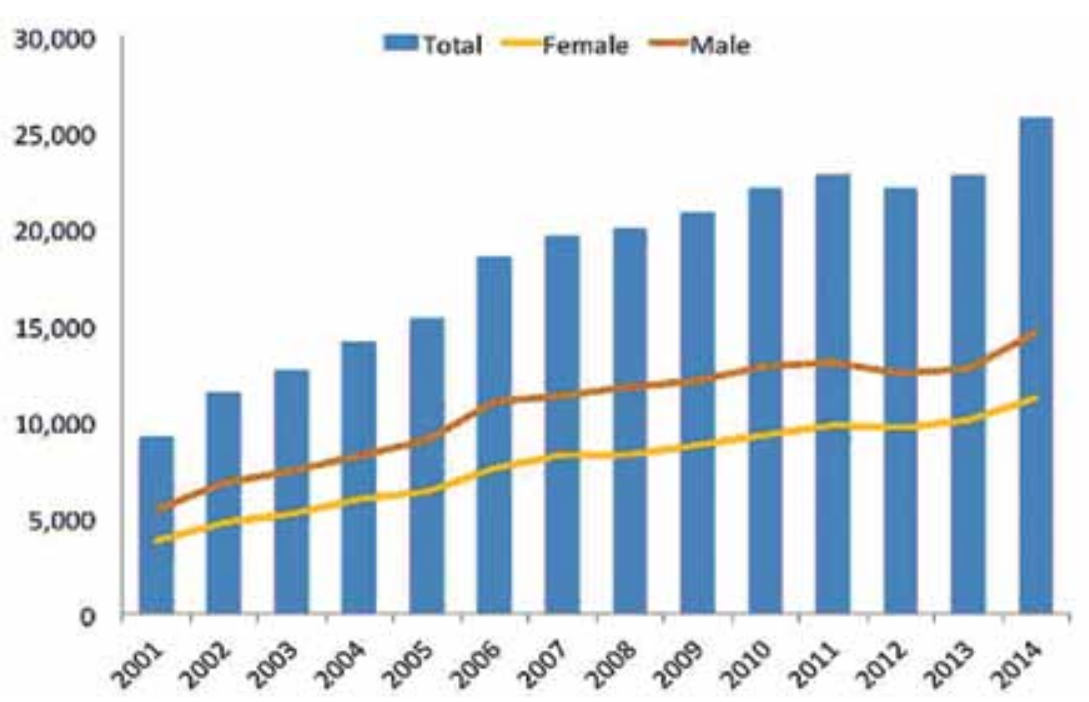

Source: National Institute on Drug Abuse. Overdose death rates. https://www.drugabuse.gov/related-topics/trends-statistics/overdose-deathrates (315)

Fig. 11. National overdose deaths: Number of deaths from prescription drugs. 
While $61 \%$ of all drug overdose deaths relate to all opioid deaths including heroin, the largest increase in the rate of drug overdose deaths involve synthetic opioids $(36,37)$. A more recent report from the CDC (37) shows that from 2014 to 2015, the death rate from synthetic opioids other than methadone, which includes fentanyl, increased by $72.2 \%$ and heroin death rates increased by $20.6 \%$ (Figs. 1-4). In contrast, in 2014 , the rate of drug overdose deaths involving natural and semisynthetic opioids (i.e., morphine, oxycodone, and hydrocodone) had increased to 3.8 per 100,000 population (36). This is the highest rate of opioid overdose deaths and was an increase of 9\% from 3.5 per 100,000 in 2013 (Fig. 12). From 2014 to 2015, opioid death rates secondary to natural and semisynthetic opioids showed an increase of $2.6 \%$ from 3.8 to 3.9 per 100,000 population; however, methadone-related deaths decreased $9.1 \%$ from 2014 to 2015 (35). Consequently, deaths due to prescription opioids from natural and semisynthetic opioids as well as methadone have increased from 15,559 in 2014 to 16,028 in 2015 , yielding an overall increase data of $3 \%$ which is a significant reduction from previous years for overall prescription drugs including methadone or for natural and semisynthetic opioids. Of note, drug-induced deaths have exceeded motor vehicle crash deaths in the United States in 2008 with increasing drug-induced deaths while motor vehicle crash deaths have reduced (Fig. 13). However, heroin overdose deaths increased by $26 \%$ from 2013 to 2014 and 20.6\% from 2014 to 2015, and have more than tripled since 2010 from 1.0 per 100,000 population to 3.4 per 100,000 population in $2014(36,37)$ (Fig. 14). Further, heroin deaths also surpassed gun homicides for the first time (Fig. 3). Thus, the explosive increase in death rates of heroin and fentanyl have led the DEA to refer to them as most significant drug-related threats to the United States (37).

Dowell et al (42), in the CDC guidelines, described that opioid-related overdose risks were dose dependent, with higher opioid doses associated with increased overdose risk as shown in Table 11 (13,316-323).

In addition to adults, hospitalizations for opioid poisonings among children and adolescents have also

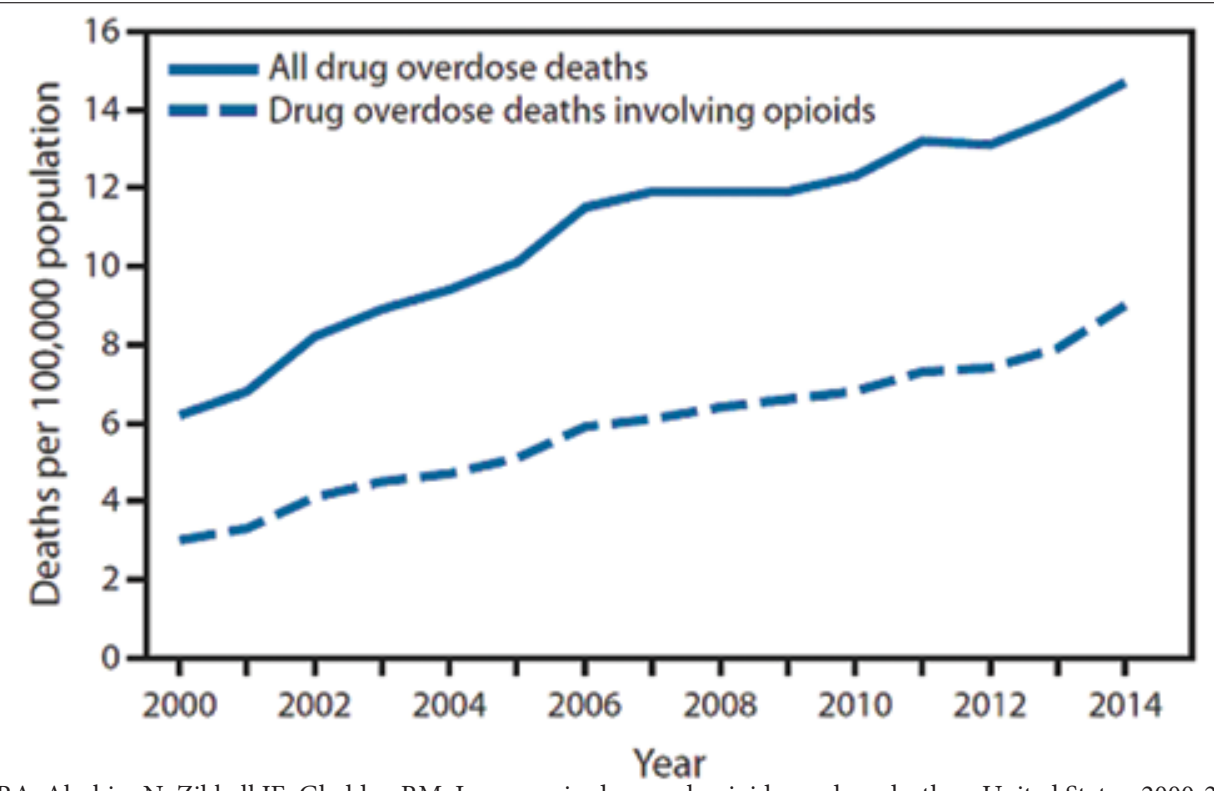

Source: Rudd RA, Aleshire N, Zibbell JE, Gladden RM. Increases in drug and opioid overdose deaths - United States, 2000-2014. MMWR Morb Mortal Wkly Rep 2015; 64:1378-1382 (37).

* Age-adjusted death rates were calculated by applying age-specific death rates to the 2000 U.S. standard population age distribution. $\dagger$ Drug overdose deaths are identified using International Classification of Diseases, Tenth Revision underlying cause-of-death codes X40-X44, $\mathrm{X} 60-\mathrm{X} 64, \mathrm{X} 85$, and Y10-Y14.

$\S$ Drug overdose deaths involving opioids are drug overdose deaths with a multiple cause-of-death code of T40.0, T40.1, T40.2, T40.3, T40.4, or T40.6. Approximately one fifth of drug overdose deaths lack information on the specific drugs involved. Some of these deaths might involve opioids.

g Opioids include drugs such as morphine, oxycodone, hydrocodone, heroin, methadone, fentanyl, and tramadol.

Fig. 12. Age-adjusted rate* of drug overdose deaths广 and drug overdose deaths involving opioids $\$$, — United States, $2000-$ 2014. 
Responsible, Safe, and Effective Prescription of Opioids for Chronic Non-Cancer Pain

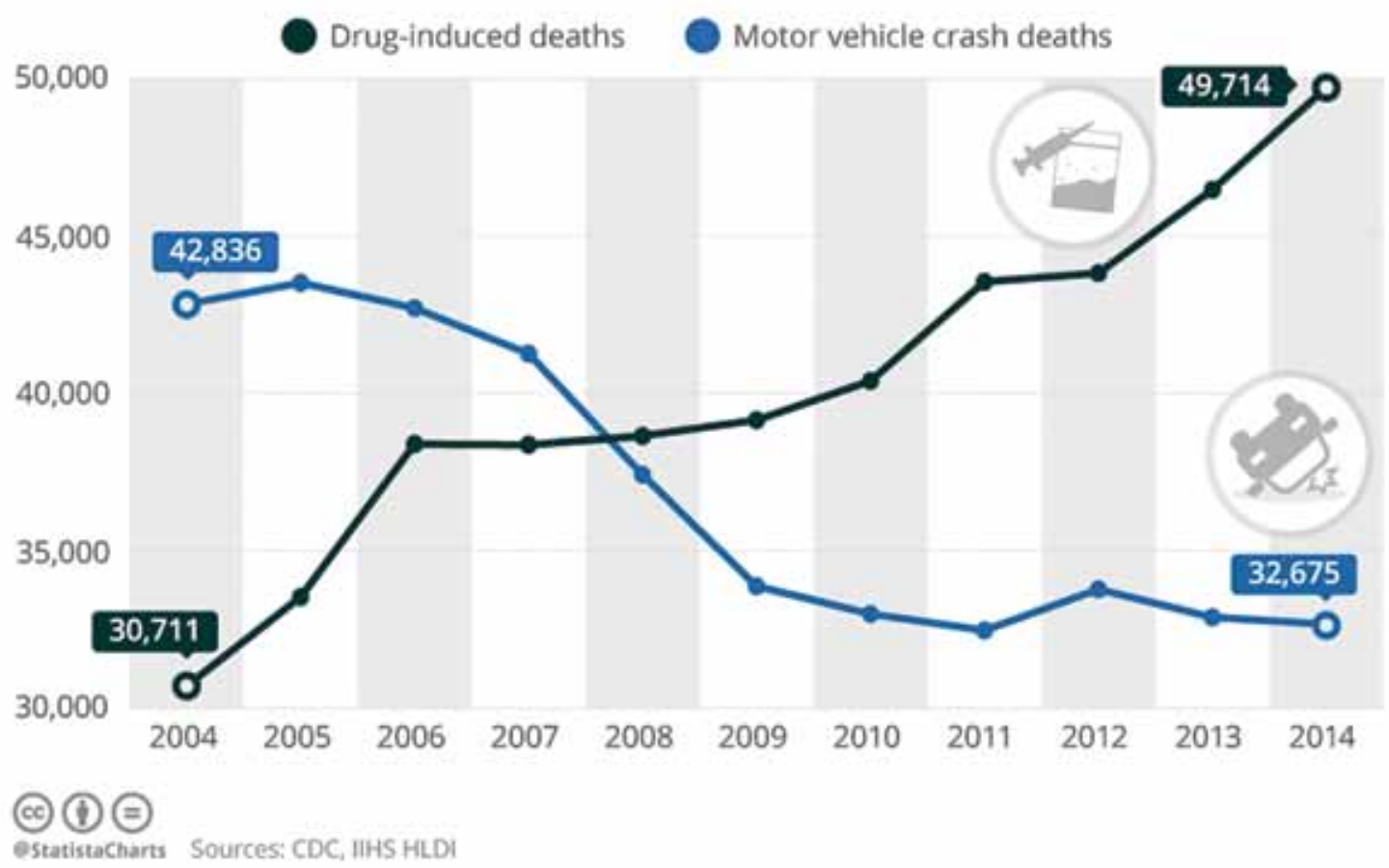

Fig. 13. Drug-induced and motor vehicle crash deaths in the U.S. from 2004 to 2014.

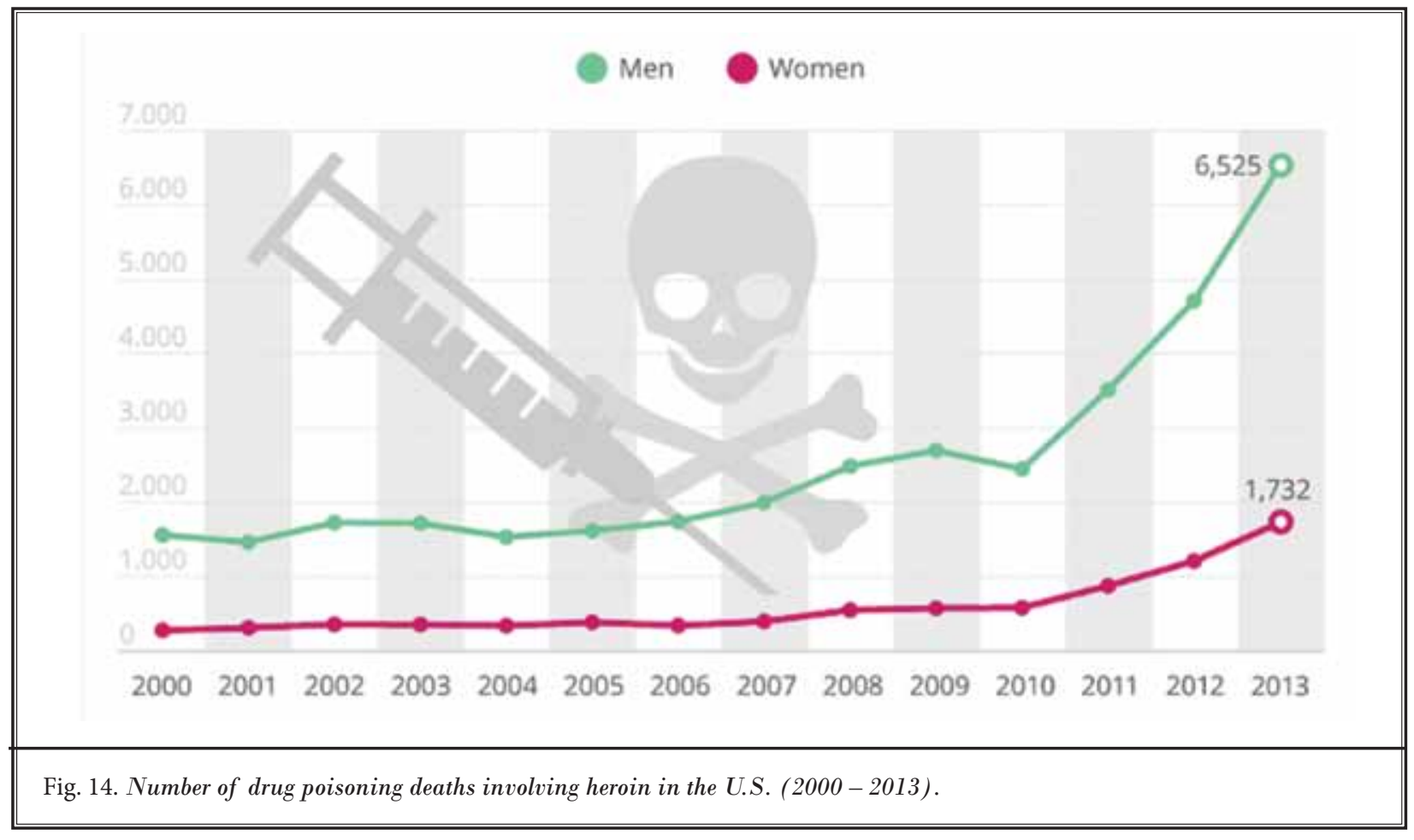


Table 11. Relationship between dose and overdose.

\begin{tabular}{|c|c|c|c|c|}
\hline Source & Topic & Population & \begin{tabular}{|l} 
Primary \\
Outcomes
\end{tabular} & Key Findings \\
\hline $\begin{array}{l}\text { Bohnert et al } \\
(321), 2016\end{array}$ & $\begin{array}{l}\text { Matched case-control study } \\
\text { examining association } \\
\text { between opioid dosage and } \\
\text { fatal overdose }\end{array}$ & $\begin{array}{l}\text { Veterans Health } \\
\text { Administration patients with } \\
\text { chronic pain receiving opioid } \\
\text { therapy, 2004-2009 }\end{array}$ & $\begin{array}{l}\text { Unintentional fatal } \\
\text { opioid overdose }\end{array}$ & $\begin{array}{l}24 \% \text { of controls had dosages }>50 \mathrm{MME} / \mathrm{d} \text {, } \\
\text { but } 59 \% \text { of cases had dosages above this } \\
\text { level. }\end{array}$ \\
\hline $\begin{array}{l}\text { Bohnert et al } \\
(317), 2011\end{array}$ & $\begin{array}{l}\text { Case-cohort study } \\
\text { examining the association } \\
\text { between prescribed opioid } \\
\text { dosage in MME/d and risk } \\
\text { of opioid overdose death }\end{array}$ & $\begin{array}{l}\text { Veterans Health } \\
\text { Administration patients } \\
\text { receiving opioid therapy for } \\
\text { pain, 2004-2005 }\end{array}$ & $\begin{array}{l}\text { Fatal opioid } \\
\text { overdose }\end{array}$ & $\begin{array}{l}\text { Among patients with chronic pain, } \\
\text { receiving } 20<50 \mathrm{MME} / \mathrm{d}, 50-<100 \mathrm{MME} / \\
\mathrm{d} \text {, and } \geq 100 \mathrm{MME} / \mathrm{d} \text { was associated with } \\
\text { adjusted HRs for overdose death of } 1.88 \text {, } \\
4.63 \text {, and } 7.18 \text { compared with } 1-<20 \\
\mathrm{MME} / \mathrm{d} \text {. }\end{array}$ \\
\hline $\begin{array}{l}\text { Dasgupta } \\
\text { et al (318), } \\
2016\end{array}$ & $\begin{array}{l}\text { Prospective observational } \\
\text { cohort study investigating } \\
\text { fatal overdose among } \\
\text { patients receiving opioid } \\
\text { pain medication }\end{array}$ & $\begin{array}{l}\text { Residents of North Carolina } \\
\text { receiving a prescription for } \\
\text { opioid pain medication }\end{array}$ & $\begin{array}{l}\text { Overdose death } \\
\text { involving opioid } \\
\text { pain medication }\end{array}$ & $\begin{array}{l}\text { Overdose risk increased steadily in } \\
\text { a dose-dependent manner; rate of } \\
\text { increase decreased after } 200 \mathrm{MME} / \mathrm{d} \text {. } \\
\text { Evidence of concurrent benzodiazepine } \\
\text { prescription in the past year was } 80 \% \text {, and } \\
\text { benzodiazepines were determined to be } \\
\text { involved in } 61 \% \text { of deaths involving opioid } \\
\text { pain medications. }\end{array}$ \\
\hline $\begin{array}{l}\text { Dunn et al } \\
(316), 2010\end{array}$ & $\begin{array}{l}\text { Cohort study examining } \\
\text { rates of opioid overdose } \\
\text { and association with opioid } \\
\text { dosage among patients } \\
\text { receiving chronic opioid } \\
\text { therapy }\end{array}$ & $\begin{array}{l}\text { Health maintenance } \\
\text { organization patients } \\
\text { who received } \geq 3 \text { opioid } \\
\text { prescriptions within } 90 \mathrm{~d} \text { for } \\
\text { chronic non-cancer pain }\end{array}$ & $\begin{array}{l}\text { Opioid-related } \\
\text { overdose (fatal or } \\
\text { nonfatal) }\end{array}$ & $\begin{array}{l}\text { Compared with receiving } 1-<20 \mathrm{MME} / \mathrm{d} \text {, } \\
\text { receiving } 20<50 \mathrm{MME} / \mathrm{d}, 50-<100 \mathrm{MME} \\
\mathrm{d} \text {, and }>100 \mathrm{MME} / \mathrm{d} \text { was associated with } \\
\text { adjusted HRs for overdose of } 1.4,3.7 \text {, and } \\
8.9 \text {. }\end{array}$ \\
\hline $\begin{array}{l}\text { Gomes et al } \\
(322), 2011\end{array}$ & $\begin{array}{l}\text { Case-control study } \\
\text { examining association } \\
\text { between opioid dose } \\
\text { level and opioid-related } \\
\text { mortality }\end{array}$ & $\begin{array}{l}\text { Ontario residents aged 15-64 } \\
\text { y who received an opioid for } \\
\text { nonmalignant pain through } \\
\text { public prescription drug } \\
\text { coverage, 1997-2006 }\end{array}$ & $\begin{array}{l}\text { Coroner's } \\
\text { determination } \\
\text { of opioid-related } \\
\text { death }\end{array}$ & $\begin{array}{l}\text { Compared with receiving } 1<20 \mathrm{MME} / \mathrm{d} \text {, } \\
\text { receiving } 20-49 \mathrm{MME} / \mathrm{d}, 50-99 \mathrm{MME} \mathrm{d} \text {, } \\
\text { and } 100-199 \mathrm{MME} / \mathrm{d} \text { was associated with } \\
\text { odds ratios for fatal overdose of } 1.3,1.9, \\
\text { and } 2.0 .\end{array}$ \\
\hline $\begin{array}{l}\text { Gwira } \\
\text { Baumblatt et } \\
\text { al (13), } 2014\end{array}$ & $\begin{array}{l}\text { Matched case-control study } \\
\text { examining association } \\
\text { between opioid dosage or } \\
\text { number of prescribers or } \\
\text { pharmacies with overdose } \\
\text { death }\end{array}$ & $\begin{array}{l}\text { Patients enrolled in } \\
\text { Tennessee Controlled } \\
\text { Substances Monitoring } \\
\text { Program, 2007-2011 }\end{array}$ & Fatal overdose & $\begin{array}{l}\text { Opioid-related overdose death was } \\
\text { associated with }>100 \mathrm{MME} / \mathrm{d}, \geq 4 \\
\text { prescribers, and } \geq 4 \text { pharmacies (adjusted } \\
\text { odds ratios, } 11.2,6.5 \text {, and } 6.0 \text { ). At least one } \\
\text { of these risk factors was present in } 55 \% \text { of } \\
\text { overdose deaths. }\end{array}$ \\
\hline $\begin{array}{l}\text { Liang and } \\
\text { Turner (319), } \\
2015\end{array}$ & $\begin{array}{l}\text { Longitudinal cohort study } \\
\text { examining association } \\
\text { between opioid dosage } \\
\text { levels and overdose }\end{array}$ & $\begin{array}{l}\text { Health maintenance program } \\
\text { enrollees who filled at least } \\
2 \text { schedule II or III opioid } \\
\text { analgesic prescriptions from } \\
\text { January } 2009 \text { through July } \\
2012\end{array}$ & Fatal overdose & $\begin{array}{l}\text { Overdose risk was associated with daily } \\
\text { opioid dosage. In addition, among patients } \\
\text { prescribed 50-100 MME/d, overdose } \\
\text { risk was significantly greater for patients } \\
\text { prescribed > 1830 MME cumulatively over } \\
6 \text { mo. }\end{array}$ \\
\hline $\begin{array}{l}\text { Paulozzi et al } \\
\text { (323), } 2012\end{array}$ & $\begin{array}{l}\text { Matched case-control study } \\
\text { examining association } \\
\text { between overdose death } \\
\text { and patterns of use of } \\
\text { opioid } \\
\text { analgesics }\end{array}$ & $\begin{array}{l}\text { New Mexico residents who } \\
\text { died of unintentional drug } \\
\text { overdoses and patients } \\
\text { with prescriptions in the } \\
\text { Prescription Monitoring } \\
\text { Program, April 2006-March } \\
2008\end{array}$ & Fatal overdose & $\begin{array}{l}\text { Patients receiving a daily average dose } \\
\text { of }>40 \mathrm{MME} \text { had a } 12.2 \text { greater odds of } \\
\text { overdose compared with those with lower } \\
\text { opioid dosages or no opioid prescriptions. }\end{array}$ \\
\hline $\begin{array}{l}\text { Zedler et al } \\
(320), 2014\end{array}$ & $\begin{array}{l}\text { Association between opioid } \\
\text { dose and overdose }\end{array}$ & $\begin{array}{l}\text { Patients dispensed an opioid } \\
\text { by the Veterans Health } \\
\text { Administration, 2010-2012 }\end{array}$ & $\begin{array}{l}\text { Respiratory/ } \\
\text { central nervous } \\
\text { system depression, } \\
\text { overdose }\end{array}$ & $\begin{array}{l}\text { Compared with patients with } 1<20 \\
\mathrm{MME} / \mathrm{d} \text {, the odds ratio of overdose was } 1.5 \\
\text { for patients prescribed } 20<50 \mathrm{MME} / \mathrm{d}, 2.2 \\
\text { for patients prescribed } 50<100 \mathrm{MME} / \mathrm{d}, \\
\text { and } 4.1 \text { for patients prescribed } \geq 100 \\
\mathrm{MME} / \mathrm{d} \text {. }\end{array}$ \\
\hline
\end{tabular}

Source: Dowell D, Haegerich TM, Chou R. CDC Guideline for prescribing opioids for chronic pain--United States, 2016. JAMA 2016; 315:1624$1645(42)$. 
been increasing (324). Gaither et al (324) identified a total of 13,052 hospitalizations for prescription opioid poisonings from 1997 to 2012. The results showed that the annual incidence of hospitalizations for opioid poisonings per 100,000 children aged 1 to 19 years increased from 1.40 to 3.7 , an increase of $165 \%$. In contrast, opioid poisonings increased $205 \%$ among children 1 to 4 years of age, whereas the increase was $176 \%$ for adolescents aged 15 to 19 years. Further, in adolescents aged 15 to 19 years, heroin poisonings increased $161 \%$, whereas methadone poisonings increased $950 \%$ (Fig. 15). The most concerning of this assessment is methadone poisonings with an increase of $950 \%$.

The systematic assessment of evidence showed that opioid doses of 200 MME per day (318) increased mortality rates gradually, higher than the doses of 100 MME or more per day (322), increasing the risks for opioid overdose by factors of 2.0 to 8.9 (317). The evidence also showed opioid doses of 50 to less than 100 MME per day were found to increase the risk for opioid overdose by factors 1.9 (322) to 4.6 (317) compared to the dosage of one to less than 20 MME per day. In addition, the absolute risk difference approximation was $0.15 \%$ for fatal overdose (317) and $1.4 \%$ for any over- dose (316). VHA patients with chronic pain also showed that patients dying of overdoses received higher doses (98 MME) per day compared to the controls receiving $48 \mathrm{mg}$ per day equivalence (321). In addition, disproportionate numbers of overdose deaths were also associated with methadone (325). A fatal overdose risk was also increased significantly with co-prescription of opioids and benzodiazepines $(316,318,326)$. Increased overdose risks were also associated with sleep disorder breathing (327-329), reduced renal or hepatic function (328,329), older age (329-331), pregnancy (327-334), mental health comorbidities, and history of substance use disorder (335-337).

Dowell et al (42), in preparation of CDC guidelines for prescribing opioids for chronic pain, showed that the evidence for long-term opioid therapy in chronic pain treatment outside of end of life care continued to be limited. There was insufficient evidence to determine long-term benefits. Dowell et al (42) were unable to do meta-analysis due to the limited number of studies, variability in study designs and clinical heterogeneity, and methodological shortcomings of studies.

The CDC also highlighted not only the increase in overdose deaths from opioids, but also a major surge in

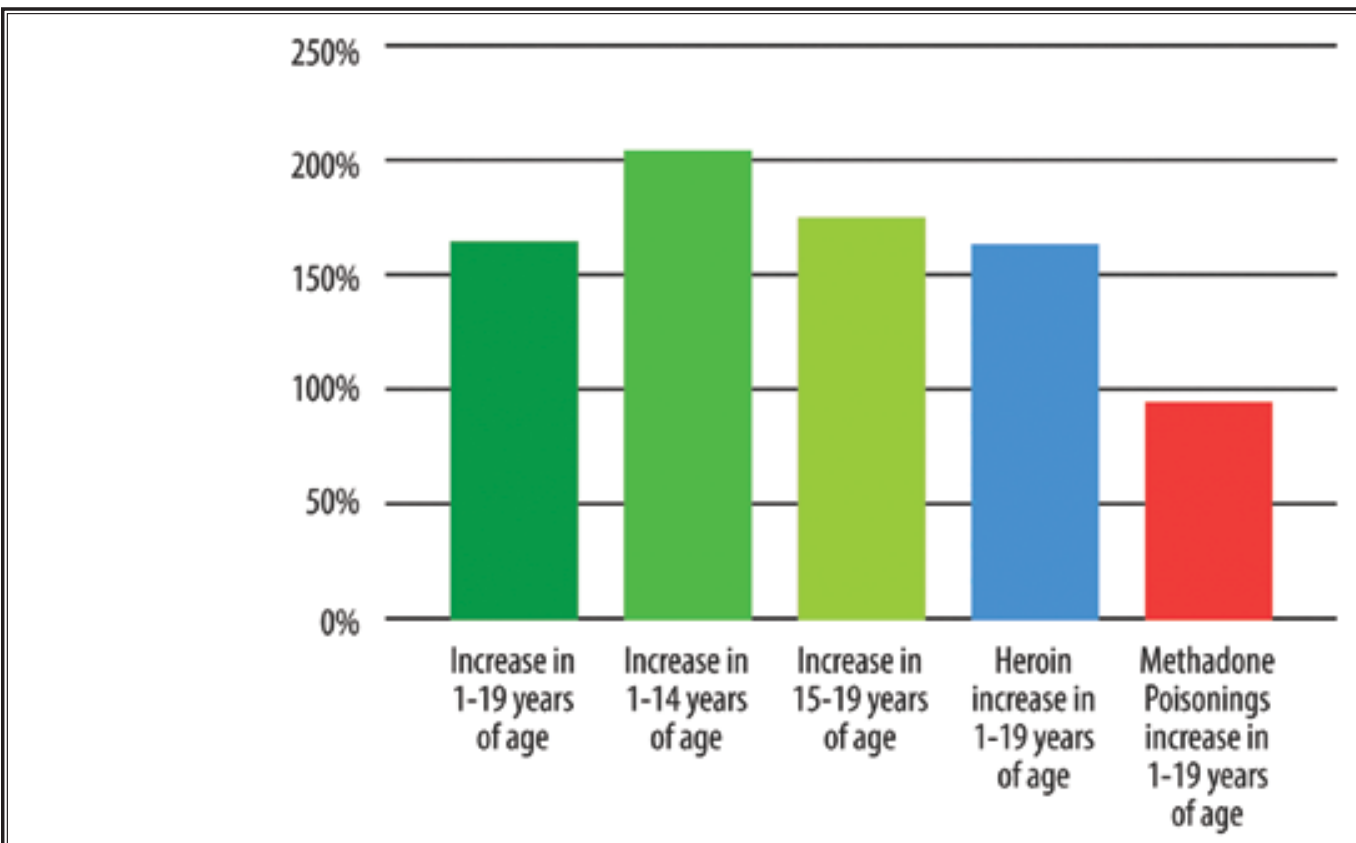

Source: Gaither JR, Leventhal JM, Ryan SA, Camenga DR. National trends in hospitalizations for opioid poisonings among children and adolescents, 1997 to 2012. JAMA Pediatr 2016 [Epub ahead of print] (324).

Fig. 15. National trends in hospitalizations for opioid poisonings among children and adolescents from 1997 to 2012. 
illicit opioid overdose deaths, driven largely by heroin. These 2 trends, while distinct and separate, are also interrelated $(2,4,52,126,149,155-159,338-347)$. Ironically, the present interpretation of overdose deaths and their available data seem to lag behind the current status. The emerging evidence shows that apart from heroin, fentanyl has become a dominating force in the escalating epidemic of lethal opioid overdose as shown in Figs. 1 - $4(36,37,52,155-159)$. Kertesz (52) described that opioid deaths continue to increase despite sustained reductions in opioid prescribing and sustained reductions in prescription opioid misuse. Kertesz (52) described that while opioid prescribing by physicians appears to have unleashed the epidemic prior to 2012, physician prescribing no longer plays a major role in sustaining it. Now illicit fentanyl and heroin are dominating the opioid overdose epidemic, along with methadone as shown in Figs 1 - 4, and 14 (36,37,348-353).

Of particular concern has been the rise in heroin abuse, specifically in the younger population. In addition, reports show that the exclusive use of heroin more than doubled from 2008 to 2014 from $4.3 \%$ to $9 \%$ (340,343). Unfortunately, the combined use of prescription opioids and heroin increased more dramatically than heroin use alone $(340,343)$. This is illustrated by the fact that the number of past year heroin users in the United States nearly doubled from 380,000 in 1999 to 670,000 in 2012 (343). There has been an increase in heroin overdose death rates of $26 \%$ year to year from 2013 to 2014 and $20.6 \%$ from 2014 to 2015 based on the CDC data $(36,37)$. Deaths more than tripled from one per 100,000 in 2010 to 3.4 per 100,000 in 2014 (36). Further, heroin poisoning is a higher risk than opioid poisoning because of its purity and injection capabilities, along with possible contamination and concomitant use with potent prescription opioids and links to transmission of HIV, hepatitis C, sexually transmitted infections, and other diseases.

In addition to heroin-related deaths, one of every 3 opioid-related deaths is associated with methadone ingestion, a substantially higher proportion than any other prescription opioid, with methadone prescriptions constituting only one-tenth of overall opioid prescriptions (348-353). This led the FDA to issue a guideline to limit methadone to $30 \mathrm{mg}$ per day for chronic non-cancer pain. Methadone also has been attributed to multiple cardiovascular complications. Lev et al (349) compared methadone-related deaths to all prescription-related deaths in a retrospective observational study with analysis of deaths in San Diego County during the year 2013. They showed that methadone- related deaths accounted for 46 out of the 254 total deaths (18.1\%); however, methadone prescriptions were found in 14 patients with Prescription Drug Monitoring Program (PDMP) reports, 10 of whom showed methadone on the toxicology report. Further, the CDC reported unintentional death from methadone increased dramatically from 790 deaths in 1990 to over 5,400 deaths in 2006, representing the fastest increase of all drug-related deaths during that time span (351). In another study related to methadone deaths in Western Virginia in 2004, Weimer et al (350) showed that the source of methadone in the 61 methadone-related overdose deaths was mostly non-prescribed (67\%). Consequently, they concluded that the majority of methadone overdose deaths in this study were related to illicit methadone use rather than prescribed in an opioid treatment program.

With the multitude of issues described above, it is understandably disturbing to know that more than $90 \%$ of patients who survive a prescription opioid overdose also continue to receive prescription opioids after the event (354). Even more surprising is that the repeat prescriptions are provided by the same provider, but at lesser doses. At the end of 2 years, the cumulative incidence of repeat overdose was shown to be $17 \%$ for patients receiving high doses of greater than $100 \mathrm{mg}$ of morphine equivalent dosage per day of opioids and $15 \%$ for those receiving moderate dose of $50 \mathrm{mg}$ to 99 $\mathrm{mg}$ of morphine equivalent doses per day. Further, even low dose therapy with less than $50 \mathrm{mg}$ of morphine equivalent doses per day showed incidence of repeat overdose of $9 \%$; however, this was similar to those receiving no prescription opioids.

Driving under the influence of drugs is an important issue with the widespread use of opioids and other psychoactive drugs $(110,141-149,152,355-361)$. Leung et al (355) presented an overview of experimental research pertaining to benzodiazepines, opioids, and driving. Overall, there is growing experimental evidence linking the therapeutic use of benzodiazepines and opioids to an increased crash risk. However, the experimental literature remains unclear with limitations of the study methodologies resulting in inconsistent findings. While psychomotor impairment following acute administration of an opioid or an increase of opioid dosage is demonstrated, impairment diminishes with chronic, stable opioid usage (357). Performance studies (356-364), while including a small number of actual driving studies, concluded that long-term treatment with opioids was associated with limited impairment of driving skills, 
presumably through development of opioid tolerance. A structured evidence-based review by Fishbain (356) in 2003 showed moderate, generally consistent evidence for no impairment of psychomotor abilities of opioidmaintained patients and there was strong consistent evidence for no greater incidence in motor vehicle violations or accidents compared to controls. However, in a study of patients on methadone and on-road driving (364), the patients were found to be less adept at parking and recorded more cautious traveling speeds than the control group. Sabatowski et al (359) described that currently there is no gold standard for assessing driving ability while receiving opioids and available data do not support the conclusion that one opioid is more favorable than others. Wilhelmi and Cohen (357) assessed 23 studies of which $70 \%$ supported the conclusion that no psychomotor impairment exists in patients on a stable opioid dosage. In another study, 7 of the 8 studies found no increase in the number of motor vehicle violations or motor vehicle accidents (356). However, public policy is highly variable based on jurisdictions from country to country and state to state. In the United States, 20 states do not allow the legal use of a prescription medication to be pleaded as a defense to driving under the influence of drugs (365), whereas 5 states allow such a defense. Considering variable global and national strategies, providers must be cognizant of the state laws.

Thus, opioid abuse has become an international problem $(1-4,6,8,17,19-22,29,30,36-38,41,61,62$, $85,87,88,127,291,338-354,366-380)$. The impact of opioid abuse is widespread with escalating economic burden of prescription opioid use, misuse, abuse, and adverse consequences (380-382). Estimates show that for nonmedical use of opioids, the costs have been $\$ 53.4$ billion yearly, including $\$ 42$ billion in lost productivity (382). In addition, opioid abusers have been shown to have health care costs that are exceedingly higher - 9 times higher than non-abusers. More worrisome is that while prescription opioid use is declining, it has been associated with heroin overdoses and poisoning, thus functioning as gateway for heroin, showing that $80 \%$ of heroin users first took prescription opioids $(162,340)$. Strassels (381) showed the economic burden of prescription opioid misuse and abuse to be around $\$ 90$ billion in 2001, which included workplace, health care, and criminal justice expenditures. Studies from the U.S. government from the Office of National Drug Control Policy (ONDCP) reported that the cost of drug abuse in the United States was \$193 billion in 2007 and continuing to escalate (338).

\subsection{Balancing Opioid Therapy and Abuse with Preventative and Monitoring Strategies: Key Questions 6 \& 7. WHAT ARE THE BEST PREVENTIVE AND MONITORING STRATEGIES TO REDUCE OR ELIMINATE ABUSE WHILE PRESCRIBING OPIOIDS? WHAT COMPRISES RESPONSIBLE OPIOID PRESCRIBING?}

Numerous policy initiatives, guidelines, and advisories, including PDMPs, restriction of prescription opioids, dose reductions, development of abuse deterrent formulations, and adherence monitoring initiatives, have been advanced through the years to curb opioid use and abuse including deaths $(4,17,29,36,37,42,50,55$, $109,124,127,130,131,138,352,367,369,370,375,376,383-$ 408). In fact, Meara et al (407), in testing the associations between prescription opioid receipt and state controlled substance laws, showed that from 2006 through 2012, states added 81 controlled substance laws. They assessed opioid use among disabled adults using Medicare administration data for fee-for-service (FFS) disabled beneficiaries from 21 to 64 years of age who were alive throughout the calendar year, which included 8.7 million person years from 2006 through 2012. They showed that opioid use among these individuals was high with $47 \%$ of beneficiaries filling opioid prescriptions with $8 \%$ having 4 or more opioid prescribers (doctor shopping), 5\% had prescriptions yielding a daily morphine equivalent dose of more than $120 \mathrm{mg}$ in any calendar quarter, and, finally, $0.3 \%$ were treated for a non-fatal prescription opioid overdose in 2012 alone. However, they observed no significant associations between opioid outcomes and specific types of laws or the number of types enacted. They showed that high dose prescription rates remained the same from 2006 to 2012 among disabled Medicare beneficiaries. This observation is in concordance with disabled beneficiaries on Medicare increasing rapidly compared to those over the age of 65 and the use of higher medical services for disabled beneficiaries due to their injuries, surgical interventions, and subsequent disability including interventional techniques and opioid therapy (409). In contrast, Chang et al (404), in the study of the impact of PDMPs and pill mill laws on high risk opioid prescribers, assessed data from July 2010 and September 2012 to identify opioid prescribers in Florida and Georgia. In this assessment, they identified 1,526 or $4 \%$ of the opioid prescribers as high risk prescribers in Florida accounting for $67 \%$ of total opioid volume and $40 \%$ of total opioid prescriptions. Following the policy implementation, Florida's high risk providers experienced large relative reductions in opioid patients and opioid 
prescriptions, morphine equivalent dose, and total opioid volume. However, low-risk providers did not experience a statistically significant relative reduction, nor did policy implementation affect the status of being highrisk versus low-risk prescribers. The Commonwealth of Kentucky, after instituting pill mill regulation, has experienced a significant decline in total opioid prescriptions and dispensing with total volume reductions of approximately $20 \%(410)$. This trend has been observed nationally also with overall approximately $12 \%$ reductions in prescriptions and approximately $20 \%$ reduction in dosages (45-47). It also led to a reduction of $20 \%$ in the utilization of total opioids in the United States (4547), reinforced by an announcement by the DEA of a reduction in opioid production (140). The DEA's reduction reflects decreased demand, not an effort by the DEA to decrease supply. Dowell et al (408) concluded that mandatory provider review and pain clinic laws reduce the amount of opioids prescribed and overdose death rates. They showed that combined implementation of mandatory provider review of state run PDMP data and pain clinic laws reduced opioid amounts prescribed by $8 \%$ and prescription opioid overdose death rates by $12 \%$. Patrick et al (385) also showed the implementation of PDMPs may prevent 600 overdose deaths nationwide per year.

While numerous guidelines already existed, the Washington State Agency Medical Directors' Group developed the Washington State Interagency Guideline on opioid dosing for chronic non-cancer pain defining the dose limits (50). These guidelines provided specific dosing guidance. They also include the recommendations as to when to seek pain management consultations, along with web-based continuing medical education and best practices. This has resulted in a decrease in opioid doses, in the percentage of patients going on to chronic opioid use, and in opioid fatalities within the Washington State Workers' Compensation, and finally, leveling of opioid poisonings (131). Despite these reductions, their results showed that methadone poisonings occurred at 10 times the rate of other prescription opioid poisonings and increased between 2006 and 2010 (131). ASIPP guidelines also provided similar guidance $(11,12)$ with specific dosing guidance and guidance on best practices. ASIPP also provided continuing education and competency certification in controlled substance management and inclusion of controlled substance education and competency as a prominent part of certification by the American Board of Interventional Pain Physicians (ABIPP) (411).
Subsequently, the CDC (42) also released guidelines along the same lines for primary care providers, which are applied in all settings. However, the differences among these guidelines is that ASIPP guidelines provide user friendly approaches with utilization of all other modalities prior to embarking on controlled substance management. The ASIPP guidelines also encourage use of other techniques to maintain opioid treatment at low doses. CDC guidance, as well as Washington State Interagency Guidelines on opioid therapy, failed to provide such accommodations (50). Cheung et al (18), in reviewing opioid prescribing guidelines of chronic pain with a systematic review and a critical appraisal, concluded that most guidelines recommended that clinicians avoid doses greater than 90 to 200 MME per day, have additional knowledge to prescribe methadone, recognize the risk of fentanyl, titrate cautiously, and in addition, reduce doses by at least $25 \%$ to $50 \%$ when switching opioids. All of the guidelines have a common thread and consensus about the need for written treatment agreements, prescription monitoring program assessments, and adherence monitoring with urine drug testing (UDT), which can mitigate risks; however, this is mostly based on observational evidence. The CDC guidelines have further advanced dose limitations, limitation on number of days of treatment in acute pain, and utilization of other modalities of treatments.

One group, known as Physicians for Responsible Opioid Prescribing (PROP), a non-profit organization with no pharmaceutical industry funding or ties, and advised by experts from general medicine, pain medicine, and addiction medicine on long-term opioid therapy, has taken a stance that opioids must be considered as off-label use after 120 days of prescription (402). Even though they have developed educational materials for clinicians, and seem to be without bias, the group members have provided conflicting opinions based on their indirect conflicts or confluence of interest, though direct conflict or confluence of interest is not demonstrated (412-416). Despite the lack of significant evidence of efficacy of long-term opioid therapy in chronic non-cancer pain, it is generally accepted that some patients improve and many of them function well at low doses (417). Thus, it is essential to maintain access to these medications and also apply appropriate risk management strategies $(11,12,41,42,49,50,55,417,418)$. As Alford (418) described, as a society, America have become overly opioid-centric in management of chronic pain. Alford eloquently stated that the groups lobbying against prescribing opioids 
for chronic pain state that the effectiveness of long-term opioid therapy has been inadequately studied, but it appears to be the case of absence of evidence rather than evidence of absence. Despite multiple measures to prevent opioid abuse, the evidence of efficacy of chronic opioid therapy in chronic non-cancer pain has not been advanced. Consequently, while scientific evidence must be provided, during this time, we continue to face questions regarding how to best address the epidemic of prescription opioid misuse now and achieve the right balance. It has been argued that regulations may not be the most effective means as they have shown some reductions in opioid use but no reductions in major adverse consequences - namely deaths related to opioids. Thus, the well intended strategies of regulation may potentially limit access to opioids permanently for patients who are benefitting or may potentially benefit from them $(11,12,41,42,49,50,55,352,402)$. Often this may be associated with arguments of physician autonomy and the economic benefit for physicians. These regulations may lead some clinicians to refuse to prescribe opioids even when they are indicated, based on the regulations which describe them as too risky or too much work. Further, it may also create a climate of mistrust between patients and their health care teams, even though regulations do provide a safety net and improve patient understanding in this aspect. Consequently, to continue to achieve the right balance of providing opioid therapy when indicated, multiple measures must be utilized with primary and secondary prevention.

\subsection{Primary Prevention}

The primary prevention is mainly dependent on education. Other aspects of primary prevention include careful initiation of opioid therapy in acute pain and limited duration of therapy. Education is the foremost strategy $(16,41,418)$. Prescriber and patient education is the most appropriate and finely tuned approach to address the opioid misuse epidemic, allowing physicians to individualize care on the basis of a patient's needs after a careful benefit-risk assessment and patients to develop an understanding of not only the benefits, but also the major adverse consequences of long-term opioid therapy related to tolerance, dependence, addiction, various adverse consequences including immunosuppression, and, finally, death. Education has been applied as the pivotal approach in chronic disease management. Education not only can empower clinicians, but the patients, and also the regulators, to make appropriate, well-informed decisions about whether to initiate, continue, modify, or discontinue opioid treatment for each individual patient at each clinical encounter, with essentially ultimate shared decision-making. Proper education probably will reduce overprescribing and at the same time ensure that patients in need retain access to opioids. Further, it will also deter patients from seeking opioids for pleasure rather than pain and function, empowered with the understanding of the adverse consequences of long-term opioid therapy. This will prevent anger from patients when a clinician determines that discontinuing opioid treatment is appropriate or when a clinician decides to reduce the dosage or does not agree to increase the dosages or frequency or add or change to stronger opioid therapy. Even though education may not appear to be a patient-centered approach or may not meet the criteria of shared decision-making, appropriate education on both sides will achieve this goal and will achieve the balance.

Education is of paramount importance as chronic pain is a complex, multifactorial, multidimensional problem, sometimes without objective evidence of tissue injury and often characterized by failure to improve functional status. Physicians attempt to manage chronic pain as an acute pain and patients seek immediate relief. This may lead to a situation where patients are desperately seeking immediate pain relief, whereas physicians are desperately avoiding opioid prescriptions because of regulatory expectations. Consequently, both physicians and patients may fail to appreciate the degree of benefit, if any, due to the lack of evidence at the present time and proven risks conferred by escalating prescriptions and their dosages in a futile attempt to obtain pain relief (418).

Clinician education must be initiated at all levels, starting with the first year of schooling, and patient education must be initiated with the first prescription. The first prescription must not be based on a brief subjective assessment or patient's desire to take medication for immediate pain relief. Multiple educational attempts have been made through multiple organizations; however, these educational efforts must be without control of pharmaceutical agencies. Educational efforts from the AMA and National Institutes of Health $(\mathrm{NIH})$, with establishment of centers of excellence in pain education for physician training with online courses, clinical guidelines, and standards, have been largely ineffective (375-380). In July 2012, a national volunteer prescription education initiative was approved by the FDA as a single shared Risk Evaluation and Mitigation Strategy (REMS) requiring manu- 
facturers of extended-release and long-lasting opioid analgesics to fund accredited education on safe opioid prescribing. This was based on the FDA curriculum. Thus far, the program has not trained the targeted number of prescribers (419). REMS have been controversial regarding their content and their effectiveness. Further, a report from the Inspector General of the Department of Health and Human Services (HHS) showed that the FDA lacks comprehensive data to determine whether REMS improves drug safety (420). The Office of Inspector General (OIG) found that only 7 of the 49 REMS met their goals. Further, the FDA has not identified reliable methods to assess the effectiveness of REMS. The OIG raised concerns about the overall effectiveness of the REMS program and made multiple recommendations. However, on a brighter note, despite not training the targeted number of prescribers, an evaluation suggested that REMS education can shift a clinician's self-reported practice towards safer, guideline-concordant care. This strategy once again reinforces the fact that comprehensive education and training in safe opioid prescribing is needed at all stages of medical education, which has been lacking. Further, this education must go beyond opioid prescribing to include comprehensive, multimodal pain management, and it must be designed for the entire health care team (418). Physicians and patients must be educated on all modalities of treatment, with appropriate acceptable evidence, and must involve physician groups without major confluence of interest.

In recent years these facts have been brought into focus, with control of initial prescriptions, rather than focus on long-term use $(34,55,108,421-428)$. Similarly, emergency room physicians have developed various guidelines to prescribe opioids in acute care settings and they also assessed the impact of guidelines (427). The guideline implementation showed a decrease in opioid prescriptions in all diagnostic groups for dental, neck, back, or unspecified chronic pain. All primary care physician and surgical organizations are now focusing on educational programs on use, abuse, and alternatives to opioid therapy. Recently, the American College of Surgeons launched an education program on opioids and surgery to improve the knowledge and management of pain in surgical patients with a focus on opioid risk and nonopioid alternatives. It is well known that patients who have been started on opioids for acute pain may become long-term opioid users (34,429-434). This has been illustrated in the survey of the Washington Post-Kaiser Family Foundation of long-term prescrip- tion opioids. This survey (34) showed that only $55 \%$ of the patients currently taking prescription opioids were started on them for chronic pain (34). In contrast, $18 \%$ were started for pain after surgery and another $23 \%$ were started for pain after an accident or injury (34). Only $2 \%$ started utilizing them for recreational use.

Thielke et al (429) showed that over $80 \%$ of the participants continued higher dose opioid use at oneyear, regardless of reported problems, concerns, side effects, pain reduction, or perceived helplessness. Thielke et al (435) also, in a prospective study of predictors of long-term opioid use among patients with chronic non-cancer pain, showed that at one year, $46 \%$ of the participants continued to use opioids. The strongest predictors of long-term opioid use were not patient or medication-related factors, but expectations about using opioids in the future. They recommended that asking about such expectations may be the easiest way to identify patients likely to continue opioid use long-term. Goesling et al (434) recently concluded that patients who were on opioids prior to their total knee or total hip replacement continued the use of opioids 6 months after their surgery. They also found that some of the opioid naïve patients became chronic opioid users after these surgeries. This was despite the fact that there was no association between persistent pain and persistent use of opioids. Hooten et al (430) assessed incidence and risk factors for progression from acute to longer term opioid prescribing. However, they showed that only $21 \%$ of patients progressed to an episodic prescribing pattern and $6 \%$ progressed to a chronic prescribing pattern. In contrast, persistent opioid use following cesarian section delivery was shown to be present in a very small proportion of patients (436).

\subsection{Secondary Prevention}

Secondary prevention includes focus on appropriate prescription patterns, adherence monitoring strategies, development of abuse deterrent technology, and opioid overdose prevention programs. Dowell et al (42) found indirect evidence for the potential utility of risk stratification and mitigation strategies for identifying risky prescribing practices, as well as behaviors. These strategies included review of PDMP data $(11,12,16,385-$ $389,403,404,408,428-432,437-445)$ and UDT (440), as well as co-prescription of naloxone (441).

The role of these preventive measures and their effectiveness has been well delineated in multiple reports $(408,442,443)$. However, one study has shown no change in the emergency department visits involving 
benzodiazepine misuse in early assessments from 11 metropolitan areas in the United States with PDMPs (408).

\subsubsection{Appropriate Prescribing Strategies}

Overall, authorities have focused on pain physicians, rather inappropriately, even though the majority of prescriptions are provided by primary care physicians (Fig. 4) $(38,446)$. In addition to focusing on the appropriate group of individuals providing the prescription, it is also essential to focus on the emphasis of avoidance of prescriptions in the short-term and also education of the patient with the first treatment. The present standards of short-term treatment of 120 days are not conducive to preventing long-term opioid use, as patients become dependent on these drugs during this period. As shown in multiple evaluations, patients are dependent as early as after 30 days, and after 120 days, the issue becomes one of maintenance of dependence and it is a formidable task to stop opioid therapy, specifically if the education they received from the physician enforces that they are in need of these opioids, and even higher doses, and on a long-term basis $(11,12,34,110,287,429-434,447)$.

\subsubsection{Adherence Monitoring Strategies}

Adherence monitoring is based on a wide spectrum of clinical and behavioral assessment of patients' and physicians' understanding of the issues and behavioral patterns, focused on mainly the adherence strategy rather than fear, hatred, and economic benefit. Adherence monitoring apart from clinical and behavioral assessment includes monitoring by PDMPs, UDT, and written agreements of compliance. While a national strategy for PDMPs does not exist as proposed by ASIPP in NASPER at present, PDMPs are statewide databases available in 49 states that monitor information about prescription controlled substances (48,385-389,403,404,408,439445,448-452). Even though NASPER has not been the favorite of many administrators and not mentioned in numerous reports, the state prescription monitoring programs funded by the Hal Rogers Prescription Drug Monitoring Program, named after Congressman Hal Rogers of Kentucky, has taken the lead in establishing the programs in all states, which has now become an important tool to prevent doctor shopping (451). Most concerned parties now agree that a national program would be ideal and is needed going forward. While the effectiveness of PDMPs continues to be discussed and debated, multiple advantages, as well as disadvantages, have been widely described with advantages superseding the disadvantages $(48,385-389,403,404,407,408,448-$ 452). In fact, an assessment by Patrick et al (385) showed that implementation of PDMPs was associated with reductions in opioid-related death rates. They estimated that if Missouri also adopted a PDMP and other states enhanced their programs with robust features, there would be more than 600 fewer overdose deaths nationwide per year, preventing approximately 2 deaths each day. The statewide data also suggests that PDMPs can be effective in reducing overdose deaths, which has been shown in multiple states $(403,408,404,437,438,444)$, including Florida with the implementation of multiple regulations including PDMPs to curtail the activities of dubious pain clinics. Assessments in Florida showed the significant influence of PDMPs $(404,437,438)$. Rutkow et al (437), in assessing the effect of Florida's PDMP and pill mill laws on opioid prescribing and use from July 2010 through September 2012, reported 12 months after implementation, the policies were associated with an approximately $1.4 \%$ decrease in opioid prescriptions, $2.5 \%$ decrease in opioid volume, and $5.6 \%$ decrease in MME per transaction. In another study, it was reported that PDMPs and pill mill laws had a significant impact on high risk opioid prescribers with relative reductions in opioid patients and opioid prescriptions per month, morphine equivalent dose, and total opioid volume. No statistically significant reductions occurred in case of low risk providers (404). A $50 \%$ decrease in oxycodone overdose deaths was reported in Florida (438). Texas's pill mill law was associated with declines in average morphine equivalent dose per transaction, monthly opioid volume, monthly number of opioid prescriptions, and monthly quantity of opioid pills dispensed, with reductions ranging from $8.1 \%$ to $24.3 \%$ across the outcomes at one year (451). In New York the success has been attributed to PDMPs with a $75 \%$ decrease in patients doctor shopping (451). Tennessee also saw a 36\% decrease (443) and Kentucky has reported a significant decrease (410) along with multiple other states (451). To summarize all the pain clinic laws and their effect on reducing opioids prescribed along with overdose death rates, Dowell et al (408) used IMS Health's National Prescription Audit and Government Mortality Data to examine the effect of pain clinic laws on other state policies for opioid prescribing and prescription opioid and heroin overdose death rates in the United States during 2006 to 2013. Their analysis revealed that the combined implementation of mandatory provider review of state run PDMP data and pain clinic laws was effective in reducing opioid prescriptions 
and overdose death rates and also showed relatively large but statistically insignificant reductions in heroin overdose death rates. The results showed a reduction of opioid amounts prescribed by $8 \%$ and overdose death rates by $12 \%$.

UDT has been described as a component of adherence monitoring programs; however, it also has been met with multiple controversies due to the explosive use of frequent UDT and genetic testing, as well as a multitude of abusive patterns. In fact, some practices have generated $80 \%$ of their revenues from UDT despite their multidisciplinary and large practice settings $(12,42,109,161,390,395-398,410,453-457)$. Even then, multiple benefits of UDT have been described $(456,457)$. Similarly, treatment agreements also have been criticized and their efficacy continue to be debated $(12,42$, $109,161,390,396,397,453,454)$.

\subsection{Screening Tools}

Multiple screening tools have been recommended for prediction and identification of aberrant drugrelated behaviors $(12,442,443,454,458)$. However, the evidence is mixed and there is no ideal or evidencebased instrument available that can screen for misuse and abuse of drugs or reliably predict the potential for substance abuse. The extensive review by Chou et al (454) showed that there is no reliable evidence on the accuracy of multiple monitoring strategies. Solanki et al (458) also showed weak evidence for the accuracy of multiple screening tools available.

\subsection{Abuse Deterrent Technology}

Abuse deterrent technology has been promoted by proponents as the measure to resolve the opioid epidemic and adverse consequences $(391,393,401,405,406$, $419,459,460)$. However, the evidence shows only a modest or lack of effect as an abuse deterrent for opioids $(391-393,401,405,406,459,460)$. Abuse deterrent technology is available for various routes of administration including inhalation, oral, intervenous, intramuscular, subcutaneous, and smoking. A major purpose of abuse deterrent technology is to include the prevention of crushing and grinding, dissolving with chemicals such as alcohol, and extraction with heat or cold.

Three types of abuse deterrent formulations (ADFs) have been described with multiple physical barriers. Polyethylene oxide, a physical barrier, prevents accidental crushing or chewing. However, sequestered aversive agents, such as niacin, may precipitate adverse events in patients who chew or crush tablets accidentally without intent of abuse, and even intact tablets may produce adverse events from an aversive component in some fully compliant patients. Use of sequestered opioid antagonists, such as naloxone, may represent a more effective approach to pharmacologically deterring abuse by rendering the opioid ineffective, even though it may precipitate an opioid withdrawal in patients who chew their tablet accidentally. Overall, the effectiveness of aberrant opioid deterrence technology is limited due to well-known disadvantages of long-acting drugs and the small proportion of prescriptions of long-acting opioids, specifically those utilizing abuse deterrent technology. However, recent advances in abuse deterrent technologies for the delivery of opioids may improve the effectiveness of this technology in preventing misuse and abuse (460). Unfortunately, these technologies do not prevent taking additional oral medications which is the most common method of abuse.

\subsection{Opioid Overdose Prevention Programs}

Recently, opioid overdose prevention programs have been highlighted, including the naloxone distribution program. However, opioid detoxification programs such as methadone clinics, buprenorphine clinics, or rehabilitation programs have also been considered as opioid overdose prevention programs in some circles. Even though there seems to be advocacy for the expansion of access to medication for addiction treatment with methadone, buprenorphine, and naltrexone, the efficacy of these modalities has not been established. Further, there is anecdotal evidence that methadone clinics generally accept patients using 30 or $40 \mathrm{MME}$ and increase them up to 300 to $400 \mathrm{mg}$ of morphine equivalence on a daily basis, which in itself will create major addiction and also lead to continuous methadone maintenance and ultimately to street drugs when it is not economically feasible. The same has been stated about buprenorphine clinics, which appear to deviate from detoxification (primary purpose) to maintenance and ultimately lead to withdrawals from the drugs, specifically based on economic conditions with buprenorphine more so than methadone. Clark et al (394), in a systematic review, assessed the effectiveness of naloxone distribution programs in reference to community opioid overdose prevention. The evidence suggested that opioid users can and will use naloxone to reverse opioid overdoses when properly trained. Further, appropriate training can be provided successfully through community-based opioid overdose prevention programs. 


\subsection{Effectiveness Of Nonopioid Pharmacological And Nonpharmacological Treatments: $K E Y$ QUESTION 7. WHAT COMPRISES RESPONSIBLE OPIOID PRESCRIBING?}

Dowell et al (42) described multiple nonpharmacological and nonopioid pharmacological treatments to be effective for chronic pain and recommended nonopioid therapy for treatment of chronic pain. Multiple studies ranged in duration from 2 weeks to 6 months $(42,204,207,208,211,461-486)$. They included various types of drug therapies including nonsteroidal anti-inflammatory agents, acetaminophen, and anticonvulsants. However, they have not included multiple interventional techniques which have been shown to be significantly effective based on high quality RCTs and appropriately conducted systematic reviews $(82,414,487$ 506). However, there also have been multiple discordant opinions with lack of efficacy (507-510).

Dowell et al (42) described cognitive behavior therapy (CBT) as having small positive effects on disability and catastrophic thinking (476). Despite the major recommendation by Dowell et al (42), a Cochrane systematic review of multidisciplinary biopsychosocial rehabilitation for chronic low back pain by Kamper et al (482) concluded that with less pain and disability obtained with biopsychosocial rehabilitation compared to those receiving usual care or a physical treatment, the effects were of modest magnitude and should be balanced against the time and resource requirements of multidisciplinary rehabilitation programs. They also showed that more intensive interventions were not responsible for effects that were substantially different from less intensive interventions. Further, they also felt that only those people with indicators of significant psychosocial impact may be referred to multidisciplinary biopsychosocial rehabilitation. In this systematic review they reviewed 41 RCTs with a total of 6,858 participants, with a highly variable methodology with quality ratings ranging from 1 to 9 out of 12 , and only 13 of the 41 included studies were assessed with a low risk of bias. The range of improvement across all time points equated to approximately 0.5 to 1.4 units on a 0 to 10 numeric rating scale for pain and 1.4 to 2.5 points on the Roland Morris Disability Scale, which is measured on a 0 to 24 scale, and appears to be a clinically insignificant outcome. Further, there were no differences on work outcomes. Exercise therapy has been recommended to reduce pain and improve function in chronic pain $(208,211,461-465)$. However, exercise therapy alone may not provide a meaningful response for any condition. Consequently, multimodal and multidisciplinary therapies may help to reduce pain and improve function more effectively than single modalities (208-211,467).

Multiple nonopioid pharmacologic treatments have been recommended, such as acetaminophen or other NSAIDs, as first line pharmacotherapy in chronic pain. Multiple other drugs also have been recommended specifically for neuropathic pain which include anticonvulsants (gabapentin or pregabalin), tricyclic antidepressants, and serotonin-norepinephrine reuptake inhibitors (SNRIs) (412,511-514). However, NSAIDs have been associated with hepatic, gastrointestinal, renal, and cardiovascular risks $(208,211,476,481,483,484,514-517)$. Consequently, acetaminophen and NSAIDs have been used less frequently in recent months due to multiple warnings from the FDA on acetaminophen toxicity, as well as NSAID toxicity $(511,517)$. Anticonvulsants also have been associated with a significant adverse effect profile. Above all, the perceived benefits of acetaminophen, NSAIDs, and anticonvulsants seem to be insignificant and have been always judged in conjunction with other treatments (518). Even though Dowell et al (42) have recommended these as first line and second line treatments and superior to opioids, the effect size of improvement appears to be small (482). Further, some modalities, such as biopsychosocial rehabilitation, are not widely applied in the United States (519).

Multiple interventional techniques have been described in managing chronic pain with discordant opinions $(82,487-510)$, often based on inappropriate evidence synthesis leading to inappropriate and often negative conclusions $(415,416,508,509)$. As a composite analysis of multiple systematic reviews, most interventional techniques have been shown to be with at least moderate evidence. Multiple systematic reviews assessing the effectiveness of epidural injections in managing chronic spinal pain with RCTs (487-496) have shown significant effectiveness of epidural injections in managing spinal pain. The evidence has been variable with somewhat of a stronger evidence for disc herniation and radiculopathy compared to spinal stenosis. However, the evidence for postsurgery syndrome and discogenic pain seems to be significant, but limited, compared to disc herniation. The RCTs are also much more prevalent for the lumbar spine, whereas these are limited RCTs for the cervical spine and there is only one trial for the thoracic spine. Others have shown cautious interpretation with moderate effectiveness, mostly on a short-term basis $(497,498,507)$. However, some assessments have provided 
contradictory evidence with lack of effectiveness (507516). These reports have been extensively criticized for their inappropriate analysis, confluence of interest, and finally, inappropriate classification of local anesthetics as placebo agents $(415,416,487-491,511-514)$. However, the studies performed abroad supported by governmental funding (520-522) from the National Health Services (NHS) and Health Technology Assessment (HTA) program have presented positive results for epidural injections, in contrast to AHRQ-sponsored studies $(520,521)$. In NHS and HTA sponsored studies, Lewis et al (513-520) showed the effectiveness of epidural corticosteroid injections. They (521) also showed the superiority of epidural injections to traction, percutaneous discectomy, and exercise therapy. Evidence based on best evidence synthesis ranged from Level II to III in managing multiple pathologies in the lumbar, cervical, and thoracic spine.

Systematic reviews of percutaneous epidural adhesiolysis also have shown significant effectiveness based on the analysis of multiple high quality RCTs $(499,500)$.

Facet or zygapophysial joint interventions have been assessed in multiple RCTs, both for diagnostic and therapeutic purposes $(489,501-504)$. The diagnostic accuracy of lumbar facet joint nerve blocks has been shown to be with Level I evidence (501), whereas in the cervical spine and thoracic spine, it has been shown to be Level II evidence. Therapeutic evidence also has been assessed in multiple systematic reviews with Level II evidence for lumbar and cervical radiofrequency neurotomy and facet joint nerve blocks and Level III evidence for lumbosacral intraarticular injections with Level IV evidence for cervical intraarticular injections. In the thoracic spine, based on the limited evidence, it was Level II for thoracic facet joint nerve blocks and Level IV for radiofrequency neurotomy for long-term improvement. Chou et al (508) concluded that there was no significant evidence for facet joint injection therapies in managing low back pain similar to the findings for epidural injections.

Simopoulos et al (505) assessed the diagnostic accuracy and therapeutic effectiveness of sacroiliac joint interventions. They showed that the evidence for diagnostic accuracy is Level II for diagnostic blocks. They also showed Level II to III evidence for cooled radiofrequency neurotomy in managing sacroiliac joint pain. The evidence for conventional radiofrequency neurotomy, intraarticular steroid injections, and periarticular injections with steroids or botulinum toxin has been shown to be Level III or IV.

Multiple systematic reviews have assessed the clinical and cost effectiveness of spinal cord stimulation in managing chronic spinal pain $(506,522-530)$. In one of the noteworthy reviews by Grider et al (506), evidence shown for the effectiveness of spinal cord stimulation in lumbar failed back surgery syndrome was Level I to II and moderate, Level II to III evidence was shown for high frequency stimulation compared to conventional stimulation with limited evidence of superiority for adaptive stimulation and burst stimulation over conventional stimulation.

In contrast, Hou et al (528), in a systematic evaluation of burst cycling of spinal cord stimulation for chronic low back and limb pain, with inclusion of 5 studies and a total of 117 patients, concluded that the evidence was fair and limited, with American Academy of Neurology (AAN) recommendation level $U$, yielding no recommendation because of insufficient evidence. This systemic review by Hou et al was performed on a cycling mode of $500 \mathrm{~Hz}$ with interval quiescence. The more novel waveforms as described by DeRidder were not included. A systematic review on the treatment of phantom limb pain with spinal cord stimulation by Aiyer et al (529), with review of 12 studies, showed mixed results due to the relatively small number of patients in each study. Consequently, the authors concluded that further research was needed to demonstrate the benefits of spinal cord stimulation for phantom limb pain. However, in a comprehensive review of spinal cord stimulation systems for chronic pain, Verrills et al (530) affirmed the overall safety, effectiveness, and a drug-free option for many chronic pain etiologies based on scientific literature.

Cost effectiveness of interventional techniques has been demonstrated for spinal cord stimulation (531), percutaneous adhesiolysis (532), and caudal epidural injections (533) in chronic recalcitrant pain presenting to interventional pain management settings after failure of conservative modalities and often surgical interventions. Cost effectiveness of drug therapy modalities also has been demonstrated for various nonopioid drug treatments, often with clinically insignificant outcomes and higher costs than interventional therapies $(518,534-543)$. A cost utility analysis showed caudal epidural injections to be effective at $\$ 2,172$ for one year of qualityadjusted life year (QALY) (533), \$2,650 for percutaneous adhesiolysis for one year of QALY (532), and (f) 6,392 for spinal cord stimulation for one year of QALY (531). In contrast, Wielage et al (537), in assessing cost effectiveness of various drugs in managing chronic low back pain with a U.S. private payer perspective, estimated an incremental cost-effectiveness ratio (ICER) of $\$ 59,473$ for duloxetine over naproxen. ICERs under $\$ 30,000$ were es- 
timated for duloxetine over the non-NSAIDs, with duloxetine dominating all strong opioids. In the same study, in subpopulations at a higher risk of NSAID-related adverse events, the ICER over Naprosyn was $\$ 33,105$ or lower. Consequently, they concluded that duloxetine appears to be a cost effective post-first-line treatment for chronic low back pain compared with all but generic NSAIDs.

In an assessment of the cost effectiveness of pharmaceutical management for osteoarthritis pain, Xie et al (536) identified 20 economic evaluations comparing pharmaceutical management for arthritis pain and reached the conclusion that all drugs under evaluation including opioids, NSAIDs, chondroitin sulfate, glucosamine sulfate, and acetaminophen were cost effective compared to the comparators according to commonly accepted or jurisdiction specific ICER thresholds of less than $\$ 50,000$ per QALY. While the overall quality of these economic evaluations was acceptable, comparability among these evaluations was limited. The outcomes in these studies included occasionally were clinically insignificant.

Cost effectiveness studies of pregabalin $(538,539)$ showed favorable results in assessing response of at least $30 \%$ improvement over baseline in pain scores and a patient global impression of change rating of improvement, and estimated the costs from the UK NHS perspective at 2008 prices using pregabalin $300 \mathrm{mg}$ and $400 \mathrm{mg}$, with a cost per QALY of (f) 23,166 to (f) 22,533. Even at this high cost, they found it to be cost effective to provide $30 \%$ improvement with pain. Cost utility analysis of NSAIDs is complicated by their adverse effects.

\subsection{Guidance For Responsible Opioid Prescribing for Chronic Mon-Cancer Pain: Key Questions 7 \& 8. What comprises RESPONSIBLE OPIOID PRESCRIBING? WHAT IS THE MANAGEMENT STRATEGY FOR LONG-TERM OPIOID THERAPY IN CHRONIC NON-CANCER PAIN?}

Over the years, multiple guidelines have described various steps for chronic opioid therapy in chronic non-cancer pain. Recent manuscripts include the CDC guidelines (42), interagency guidelines (50), and Canadian guidelines (51). ASIPP also has provided steps for chronic opioid therapy. However, it has been difficult to assess the effect of any published guidelines. In fact, many $(33,544,545,547,548)$ believe that the recently published CDC guidelines will hinder access to patient care, while others believe that they are unlikely to affect opioid prescribing practices (549). Multiple physician and patient groups have opposed the CDC guidelines in a non-scientific survey conducted by the Pain News Network (545). In addition, of further significance are the results of the Washington Post-Kaiser Family Foundation national poll of long-term opioid users (34) which revealed public perceptions of the regulatory atmosphere and also the effectiveness of opioids in relieving chronic pain. The survey also revealed improvement in physical function and made a significant difference in their lives. This is in contrast to the concepts of the scientific community and the guidelines which predominantly focused on curbing the opioid abuse, which may have led to denial of opioids for those with medical necessity (2$4,9-13,16,23,24,36,37,41,42)$. The survey results showed that as many as $92 \%$ opined that opioids reduced their pain at least somewhat well, whereas $53 \%$ stated that "opioids reduced their pain very well." Further, 57\% also stated that their QOL was better than if they had not taken the medications. Opioids impact on physical health and work was not so positive with $20 \%$ stating that it had a positive impact on their mental health and another $20 \%$ stating that it had a negative impact on their mental health, with $60 \%$ describing no impact. Similarly, $68 \%$ described opioids as having no impact on their personal relationships, while $15 \%$ reported a positive effect and $16 \%$ a negative effect. The failure to improve physical and functional status is quoted in the literature repeatedly $(73,91,281-292)$. However, these 2 opposing facts may be reconciled based on the fact that $70 \%$ of long-term opioid users were disabled or suffered from a handicap or chronic disease which kept them from participating fully in work, school, housework, or other activities. Consequently, clinical effectiveness, cost utility, and cost effectiveness studies have shown interventional therapies to be more economical than many of the drug therapies described in CDC guidelines (42), with interventional therapies showing clinically significant improvement utilizing criterion standards of $>50 \%$ improvement (487-496,499-506,521,525,531-533). Fifty-eight percent of the patients agreed that opioids are prescribed appropriately, 67\% agreed that opioids are a preferred treatment, and an overwhelming $89 \%$ were worried that they would not be able to get opioid pain medication. Further, $38 \%$ of the participants in the survey felt that the CDC guidelines would only increase addiction and overdoses, with only a $5 \%$ feeling that it will decrease them (545). The survey results from the Washington Post-Kaiser Family Foundation national poll (34) also showed apprehension on the part of the patients in reference to the efforts to decrease abuse of prescription opioids which could make it even more dif- 
ficult to obtain them, as $60 \%$ also believed that prescription opioids are difficult to obtain for medical purposes as it is. In contrast, a positive impact of an opioid prescribing guideline in the acute care setting also has been described (427). These authors concluded that an opioid prescribing guideline significantly decreased the rates at which opioids were prescribed for minor and chronic complaints. The overall decrease in opioid use patterns, and the decision by the DEA to reduce the production of opioids, shows that both guidelines and regulations have had a significant effect on opioid dispensing, which seems to be reduced by $25 \%(139,140)$. However, this does not seem to translate into reduction in adverse consequences, as opioid deaths continue to increase. In fact, while the Washington guidelines claim reductions in opioid prescriptions and opioid usage $(118,130)$, methadone poisonings continue to occur at 10 times the rate of other prescription opioid poisonings and increased between 2006 and 2010, with a leveling off of other opioid poisonings after guideline implementation in 2007. Investigators (131) have suggested that it may be prudent to revise guidelines to address those opioid poisonings that occur at relatively low prescribed doses and with acute and intermittent opioid use. They also alluded to the fact that research is needed to establish the best strategies to prevent opioid poisonings. Assessment of changes in opioid prescribing for chronic pain in Washington State (550) showed less concern with opioid prescribing guidelines among the providers if they were affiliated with a health care organization and had access to pain consultation; whereas, overall prescribing providers in Washington State reported ongoing concerns regarding opioid use for chronic non-cancer pain. All the guidelines for chronic opioid therapy provide recommendations to improve safety, with multiple steps recommended for implementation.

Of importance, Dowell et al (42) provided 12 recommendations, from initiation to discontinuation of opioid therapy in chronic pain. Three recommendations were included for determining when to initiate or continue opioids for chronic pain; 4 recommendations were included for determining opioid selection, dosage, duration, follow-up, and discontinuation; and 5 recommendations were included for assessing risks and addressing harms of opioid use. Similar to these recommendations, ASIPP guidelines have also provided 11 recommendations (12). The updated assessment of evidence for responsible, safe, and effective prescription of opioids in chronic non-cancer pain provides this guidance.

In these guidelines, 4 recommendations are includ- ed for the determination of initiation and continuation of opioid therapy for chronic pain: opioid selection, duration, follow-up, and discontinuation included. In addition, assessing risks and addressing harms of opioid use are also included. These recommendations coincide with recommendations by multiple other guidelines, specifically of those by CDC (42).

\subsection{Comprehensive Assessment}

\subsubsection{Pain Condition}

A thorough history and physical examination must be documented to determine the type, cause, and nature of the pain, including questions about past investigations and interventions for pain. This history also should include medication trials and the pain intensity and the functional impairment that arises from it (i.e., impact of pain on activities of daily living, work, and other aspects of life). In addition, various circumstances that increase or exacerbate the pain, and those conditions that lead to diminution of pain must be documented (12,42,551-565). A physical diagnosis must be established prior to initiating opioid therapy. The diagnosis should not be non-specific, such as low back pain, knee pain etc., but should be objective and somewhat specific, based on the type of pain and abnormalities identified. This will assist in future treatments based on whether the pain is nociceptive, neuropathic, somatic, radicular, a combination of these, widespread, or localized. The presence and extent of emotional pain also needs to be considered.

\subsubsection{General Medical History}

General medical history includes questions about general physical health, emotional health, and medication usage $(12,42,110,28-283,551-567)$. Chronic pain patients tend to have multiple medical comorbid conditions, which may increase the pain levels, decrease functional status, or interact with drug therapy $(12,42,110,282-283,551-567)$.

\subsubsection{Psychosocial History}

Psychosocial history may include information regarding their upbringing, family and social support, family obligations, work status, use of alcohol and tobacco, and living arrangements. Additional information of importance may include the intricacies of the family network, history of drug use and abuse among other family members, as well as their independence and ability to control medications. Further, physicians 
should also be cognizant of the risks associated with opioids, especially in combination with other psychoactive drugs, and the role of their spouse or family members in relation to surrogate decision-making.

\subsubsection{Functional Status}

Chronic pain invariably affects functional status, activities of daily living, and work status. Many patients suffer with significant disability (60-78,83-88). Further, disability continues to increase despite increasing use of numerous treatment modalities, including opioids $(12,42,91,281-292,300,568)$. A history of the functional status of a patient includes information about their ability to perform activities of daily living, work, play, and socialization. Baseline functional assessment and goals of treatment will be crucial in assessing progress throughout the treatment. Assessment may be performed utilizing the Oswestry Disability Index, Neck Disability Index, or other measures $(569,570)$.

\subsubsection{Sleep Patterns}

Sleeping is an important function, which is often disturbed in individuals with chronic pain. Lack of an appropriate sleep pattern can induce many harms, including fatigue, daytime sleepiness, and cognitive dysfunction. Assessment of sleep disturbances is important in patients with chronic pain problems, specifically in the elderly. Furthermore, opioid therapy, along with other psychoactive drug therapy, may induce or exacerbate multiple sleep disturbances (571-577). Thus, conditions related to sleep disturbances, including that of obstructive sleep apnea syndrome, are crucial in assessing the patient condition prior to initiation of opioid therapy (571-577). Assessment of obstructive sleep apnea syndrome is one of the neglected issues in chronic opioid therapy (578). Opioid therapy around the clock in obstructive sleep apnea patients may be deleterious. Thus, it is essential to assess for the presence and severity of obstructive sleep apnea in patients considered for opioid therapy, and also to provide appropriate control measures of sleep apnea syndrome, along with appropriate limitation of opioid administration in these patients during the night.

Risk factors for sleep disorders also include congestive heart failure and obesity. Careful monitoring and cautious dose titration should be used if opioids are prescribed for patients with mild sleep-disorder breathing; however, Dowell et al (42) recommended that clinicians should avoid prescribing opioids to patients with moderate or severe sleep-disorder breathing whenever possible, to minimize the risks for opioid overdose, based on contextual evidence.

\subsubsection{Psychological Evaluation}

A significant proportion of patients with chronic pain also suffer with multiple psychological conditions, specifically depression and anxiety $(12,110,567,579-$ $602)$. Numerous investigators have shown the relationship between psychological and emotional distress and increased levels of pain-related disability, chronicity, and use of health care services (587-591,593,595,600602). Symptoms of depression and anxiety also have been studied regarding long-term physical health outcomes in rheumatoid arthritis (596), showing that baseline and persistent symptoms of depression and anxiety were associated with poorer health outcomes over time, as well as reduced treatment response. The role of psychological status also has been described in outcomes of surgical, interventional, and non-interventional techniques $(579-585,597,598,599,603,604)$. Psychological treatment with inclusion of pharmacological therapy or psychotherapy is one of the components of effective chronic pain management. An increasing role has been assigned to behavioral and psychological therapies in managing chronic pain in recent years $(12,42,584-592)$.

In addition to common conditions, patients may also suffer with multiple other comorbidities including posttraumatic stress disorder (PTSD), multiple personality disorders, attention deficit hyperactivity disorder (ADHD), and schizophrenia. Psychological evaluation may be performed with a simple evaluation for depression, anxiety, and somatization. Patients with major personality disorders will need further evaluation and appropriate consultations (600-603).

Psychiatric status includes information regarding the patient's current and past history of psychiatric disorders and treatments and family history of psychiatric disorders.

\subsubsection{Substance Use History}

Substance use disorders, or specifically opioid use disorders, are crucial to identify in managing chronic pain and determining appropriateness of chronic opioid therapy $(12,454)$. Clinicians should assess patients for opioid use disorder using DSM-5 criteria (586). Substance use history is elicited with the inclusion of multiple questions in reference to current, past, and family history of substance use, abuse, and addiction to alcohol, tobacco, prescription drugs, street drugs, illicit drugs, over-the-counter medications, solvents, etc. 
Current substance abuse history may be assessed with review of PDMP data, medical records, and UDT. However, it is also essential to elicit past and family history of substance use disorders. Furthermore, in those patients with a history of substance abuse disorders and treatment, compliance issues should be investigated.

\subsubsection{Addiction Risk Screening}

Before initiating opioid therapy, an abuse evaluation is part of the comprehensive assessment, which also includes a thorough review of the patient's alcohol and other substance use. The history is important in assessing the patient's risk for opioid misuse or addiction. A physician may consider using a screening tool to determine the patient's risk for opioid addiction. Most of the screening tools have not been studied in depth, validated, or been compared to each other. Thus, the evidence is limited as to their reliability $(12,42,442,443,454,458,604)$

\subsubsection{Assessment of Previous Therapy}

Patients with chronic pain should receive treatment that provides the greatest benefits relative to risks (42). The CDC guidelines have described that many nonpharmacological therapies, including physical therapy, weight loss for osteoarthritis, psychological therapy such as cognitive behavioral therapy, and certain interventional procedures can ameliorate chronic pain. Consequently, it is essential to assess all treatment modalities utilized in the past, including conservative modalities as well as surgical interventions as stand alone treatments or in a multidisciplinary setting. The review of patient characteristics shows that the majority of patients have already tried extensive drug therapy, chiropractic management, and occasionally physical therapy, interventional techniques, and surgical interventions.

\subsubsection{Prescription Monitoring Programs}

Before initiating opioid therapy on any patient, a physician must obtain data from the PDMP. If a PDMP is not available, such as in Missouri, the physician may request information from all previous physicians as well as any pharmacies that a patient uses or has used. While the evidence shows a general lack of reliability and accuracy for the multiple screening tools for opioid abuse, there is good evidence that PDMPs provide data on patterns of prescription usage, and fair evidence that PDMPs can reduce prescription drug abuse or doctor shopping (385-389,403,404,407,408,437-444,448-452).

While Dowell et al (42) were unable to find the clinical evidence evaluating the effectiveness of PDMPs on outcomes related to overdose, addiction, abuse, or misuse, they concluded that since most fatal overdoses were associated with patients receiving opioids from multiple prescribers and/or with patients receiving high total daily opioid doses, information obtained from PDMPs can be crucial. However, recent assessments by Patrick et al (385) and Dowell et al (408) have shown that PDMPs, along with pill mill regulations, have reduced opioid prescriptions, opioid dosages, and opioid deaths in a significant proportion of the population.

PDMPs collect state-wide data regarding prescription drugs and track their flow. There are 3 components of these programs. The first component involves collecting data for prescriptions, documenting the physicians who wrote them and the pharmacies that dispensed them. With the enactment of the NASPER Act, physicians will have access to a database that has the capacity to monitor all transactions (48). In fact, some states are already mandating such use of PDMPs. To date, in the United States, 49 states have functioning PDMPs, except Missouri.

Clinicians should review PDMP data for opioids and other controlled medications that patients might have received from additional prescribers to determine whether a patient is receiving high total opioids dosages or dangerous combinations, as well as duplicate prescriptions or prescriptions obtained by doctor shopping. CDC recommendations state that, ideally, PDMP data should be reviewed before every opioid prescription. It may not be feasible to review PDMPs with each prescription; however, they should be reviewed at least once in 3 months, as some states have mandated. Clinicians must also share the PDMP data with patients and discuss safety concerns, not only with the patients, but also with other clinicians. The CDC has recommended that patients at increased risk from high dose opioids, prescriptions from multiple physicians, or combinations of opioids and benzodiazepines should receive naloxone. Clinicians also should consider the possibility of substance use disorder and discuss concerns with their patients (42). Even though PDMPs are useful tools, the clinician should consider errors as well as other legitimate causes of multiple prescriptions, and should not dismiss patients automatically from the practice, which may adversely effect patient safety, represent patient abandonment, and also result in missed opportunities to provide potentially life-saving information and interventions with naloxone or effective treatment for substance use disorders (42). However, after multiple warnings and continued aberrant behaviors, patients 
may be discharged when dangers of withdrawal or other adverse consequences are not likely.

\subsubsection{Urine Drug Screening}

When initiating and maintaining chronic opioid therapy, UDT should be used to establish a baseline measure of risk and to monitor compliance $(12,42,390,395-$ $398,453-457,604,605)$. It is essential for physicians to understand the pharmacology, pharmacodynamics, and drug interactions of opioids, and to have knowledge of interpretation as well as a plan in place to use the results, without financial considerations as the driving force (12).

UDT has been described in multiple manuscripts, and can provide information about drug use that is not reported by the patient or available from PDMPs. Furthermore, UDT can assist clinicians in identifying when patients are not taking opioids or other drugs as prescribed, which might indicate either overuse, misuse, or abuse, or in some cases diversion as well as other difficulties with adverse effects (42). However, UDT does not provide accurate information in reference to how much or what dose of opioids or other drugs are being consumed. There are no studies showing the effectiveness of UDT for risk mitigation during opioid prescribing for pain; however, multiple studies have shown that UDT not only provides useful information about nonprescribed or illicit drugs, but also improves compliance $(390,395,453,456,457)$. However, UDT results can be subject to misinterpretation and might sometimes be associated with practices that can harm patients with stigmatization or inappropriate termination from care. Consequently, the routine use of UDT with standardized policies will de-stigmatize their use. Randomized drug tests may be more appropriate; however, true random testing may be difficult in clinical practices. The disadvantages of UDT includes overuse and misuse, associated with additional direct costs to insurers as well as patients. Thus, a prudent policy to test patients based on their compliance is necessary. Multiple guidelines have been created by insurers and others. There is a general consensus that UDT must be used before initiating opioid therapy for chronic pain, and testing at least annually for all patients is a reasonable approach. While some experts noted that this interval might be too long in some cases, the follow-up interval should be left to the discretion of the clinician based on risk factor analysis. Previous ASIPP opioid guidelines have recommended initial testing followed by testing during the first follow-up period, and subsequently testing based on the results of the first 2 tests, as well as also other interval issues, including the results of PDMP anonymous reports and aberrant behaviors. Multiple guidelines have recommended more frequent UDT may be appropriate for patients with high risk for substance use disorder or inappropriate behaviors.

In most situations, the initial UDT can be performed with a relatively inexpensive immunoassay panel for commonly prescribed opioids and illicit drugs $(12,42)$. The use of confirmatory testing invariably adds substantial costs, and should be based only on the need to detect specific opioids that cannot be identified on standard immunoassays or on the presence of unexpected urine drug test results. Metabolite assays may also identify poor metabolism of opioids, either from genetic issues or drug-drug interactions $(11,12)$. Clinicians should familiarize themselves with the drugs included in UDT panels. Table 12 shows the interpretation of unexpected results of urine drug screens.

\subsubsection{Recommendations}

1. Comprehensive assessment and documentation is recommended before initiating opioid therapy, with documentation of comprehensive history, general medical condition, psychosocial history, psychiatric status, and substance use history. (Evidence: Level I; Strength of Recommendation: Strong)

2. Screening for opioid abuse is recommended, as it will potentially identify opioid abusers and reduce opioid abuse. (Evidence: Level II-II; Strength of Recommendation: Moderate)

3. PDMPs must be implemented as they provide data on patterns of prescription usage, potentially reducing prescription drug abuse or doctor shopping. PDMPs may reduce emergency room visits, drug overdoses, or deaths. (Evidence: Level I-II; Strength of Recommendation: Moderate to strong)

4. Presumptive UDT is implemented at initiation of opioid therapy, along with subsequent use as adherence monitoring, using in-office point of service testing, followed by confirmation with chromatography/mass spectrometry for accuracy in select cases, to identify patients who are non-compliant or abusing prescription drugs or illicit drugs. UDT may decrease prescription drug abuse or illicit drug use when patients are in chronic pain management therapy. (Evidence: Level III; Strength of Recommendation: Moderate)

\subsection{Establishing Diagnosis}


Table 12. Interpreting unexpected results of urine drug screens.

\begin{tabular}{|c|c|c|c|}
\hline \multicolumn{2}{|c|}{$\begin{array}{l}\text { Unexpected Result } \\
\text { from Presumptive } \\
\text { Screen }\end{array}$} & \multirow{2}{*}{\begin{tabular}{|l} 
Possible Explanations \\
- False negative. \\
- Non-compliance. \\
- Diversion.
\end{tabular}} & \multirow{2}{*}{$\begin{array}{l}\text { Actions for the Physician } \\
\text { - Repeat test using a confirmatory test such as chromatography/mass spectrometry; } \\
\text { specify the drug of interest (e.g. oxycodone is often missed by immunoassay). } \\
\text { - Take a detailed history of the patient's medication use for the preceding } 7 \text { days } \\
\text { (e.g., could learn that patient ran out several days prior to test). } \\
\text { - Ask patient if they've given the drug to others. } \\
\text { - Monitor compliance with pill counts. }\end{array}$} \\
\hline 1 & $\begin{array}{l}\text { UDS negative for } \\
\text { prescribed opioid. }\end{array}$ & & \\
\hline 2 & $\begin{array}{l}\text { UDS positive for } \\
\text { non-prescribed } \\
\text { opioid or } \\
\text { benzodiazepines. }\end{array}$ & $\begin{array}{l}\text { - False positive. } \\
\text { - Patient acquired opioids from } \\
\text { other sources (double doctoring, } \\
\text { "street"). }\end{array}$ & $\begin{array}{l}\text { - Repeat UDS regularly. } \\
\text { - Ask the patient if they accessed opioids from other sources. } \\
\text { - Assess for opioid misuse/addiction. } \\
\text { - Review/revise treatment agreement. }\end{array}$ \\
\hline 3 & $\begin{array}{l}\text { UDS positive for } \\
\text { illicit drugs (e.g., } \\
\text { cocaine, cannabis). }\end{array}$ & $\begin{array}{l}\text { - False positive. } \\
\text { - Patient is occasional user or } \\
\text { addicted to the illicit drug. } \\
\text { - Cannabis is positive for patients } \\
\text { taking dronabinol (Marinol }{ }^{\circ} \text {, } \\
\text { THC:CBD }\left(\text { Sativex }{ }^{\circ} \text { ) or using }\right. \\
\text { medical marijuana. }\end{array}$ & $\begin{array}{l}\text { - Repeat UDS regularly. } \\
\text { - Assess for abuse/addiction and refer for addiction treatment as appropriate. } \\
\text { marijuana access program. }\end{array}$ \\
\hline 4 & $\begin{array}{l}\text { Urine creatinine } \\
\text { is lower than } 2-3 \\
\text { mmol/liter. }\end{array}$ & - Patient added water to sample. & $\begin{array}{l}\text { - Repeat UDS. } \\
\text { - Consider supervised collection or temperature testing. } \\
\text { - Take a detailed history of the patient's medication use for the preceding } 7 \text { days. } \\
\text { - Review/revise treatment agreement. }\end{array}$ \\
\hline 5 & $\begin{array}{l}\text { Urine sample is } \\
\text { cold. }\end{array}$ & $\begin{array}{l}\text { - Delay in handling sample } \\
\text { (urine cools within minutes). } \\
\text { - Patient added water to sample. }\end{array}$ & $\begin{array}{l}\text { - Repeat UDS, consider supervised collection or temperature testing. } \\
\text { - Take a detailed history of the patient's medication use for the preceding } 7 \text { days. } \\
\text { - Review/revise treatment agreement. }\end{array}$ \\
\hline
\end{tabular}

UDS = urine drug screen; THC = Tetrahydrocannabinol $\mathrm{CBD}=$ cannabidiol

Source: Canadian Guideline for Safe and Effective Use of Opioids for Chronic Non-Cancer Pain® 2010 National Opioid Use Guideline Group (NOUGG) (314).

Diagnosis may be established by various means, including physical examination, x-rays, magnetic resonance imaging (MRI), computed tomography (CT), and neurophysiologic studies. Furthermore, psychological evaluations and precision diagnostic interventions may also be applied. Diagnostic interventional techniques will assist in making the proper diagnosis by following an algorithmic approach. Research shows that in approximately $70 \%$ to $85 \%$ of patients with spinal pain, an accurate diagnosis may not be provided even with the available history, physical examination, electromyographic/nerve conduction studies (EMG/NCV), and radiologic evaluation (11,12,501,505,606-640). With precise diagnostic interventional techniques, the chances of an accurate diagnosis may be improved substantially, and proper treatment may be offered. Once the diagnosis is established, various modalities of therapy may be offered with interventional techniques or other techniques. Whatever opioids are required should be prescribed in low doses.

Given the degree to which routine imaging has been criticized, it may be appropriate that physicians follow the recommendations provided by professional organizations and governmental organizations. When ordering and interpreting various investigations, being conservative may be prudent, due to findings in asymptomatic patients and also the psychological factors and nocebo effect introduced in these patients by graphic descriptions of asymptomatic abnormalities (413-416,472,613-640). Guidelines provided by specialty societies are appropriate if they were peer-reviewed and developed utilizing guidance from IOM criteria. Early imaging is discouraged in all circles. It is also crucial to realize that numerous abnormalities are generally found on imaging in asymptomatic patients (624-632,635-640). In the era of information disclosure and electronic media, findings that do not correlate with symptoms and do not provide certainty as a pathological entity should be addressed only by qualified physicians, not by technologists and radiologists who have no clinical correlation. Irrelevant and non-corroborative findings create fear and activity avoidance, resulting in negative consequences 
including requests for increased opioid dosages.

The role of neurophysiological testing is limited in chronic pain management, even though some insurers mistakenly focus on the neurophysiological evaluation and findings $(614-618,632)$.

\subsubsection{Consultation(s)}

Physicians should be willing to refer a patient as clinically indicated for additional evaluation to achieve treatment objectives. Special attention should be given to those patients who are at risk of misusing their medications and those whose living arrangements create a risk for medication misuse or diversion. The management of patients with a history of substance abuse or with a coexisting psychiatric disorder may require extra care, monitoring, documentation, as well as consultation with, or referral to, an addictionologist. The lack of well-trained psychologists and psychiatrists in chronic pain management in many regions of the country may make this referral difficult to obtain. Likewise, in many locations there are no clinically trained addiction specialists with whom to collaborate.

\subsubsection{Recommendations}

1. Establish appropriate physical diagnosis and psychological diagnosis if available prior to initiating opioid therapy. (Evidence: Level l; Strength of Recommendation: Strong)

2. Appropriate imaging should be used based on the physical exam and objective findings that suggest further imaging is needed. Abnormal imaging should be evaluated to assess whether it correlates with subjective complaints. (Evidence: Level Il; Strength of Recommendation: Moderate)

3. A pain management consultation, for non-pain physicians, if use of chronic opioids is planned or in those who exceed the recommended CDC morphine equivalent therapy. (Evidence: Level III; Strength of Recommendation: Moderate)

\subsection{Stratification of Risk}

Stratification of risk for patients initiated or maintained on chronic opioid therapy is crucial to prevent misuse and abuse. These principles may also be applied for patients who are treated for acute pain management, but also have other risk factors and for whom pain may become chronic. Chronic opioid therapy has been defined as therapy lasting for at least 90 days, on a daily or near daily basis (641). Consequently, all guidelines recommend that, before initiating chronic opioid therapy for any patient and in high-risk patients for acute pain therapy, a clinician should conduct a history, physical examination, and appropriate testing, including an assessment of risk of substance abuse, misuse, or addiction. Atluri et al (642) describe risk stratification of patients into different categories as the first step. This risk stratification is justified in all patients due to the significant proportion of misuse and abuse $(12,42,442,443,454,642-646)$. Chou et al (454) described that risk stratification pertaining to outcomes associated with abuse liability of opioids - misuse, abuse, addiction, and diversion - is a vital but relatively undeveloped skill for many clinicians $(12,152,421,422)$. All clinicians prescribing opioids, however, should be knowledgeable about the risk factors for opioid abuse. Moreover, it is also essential to perform an assessment of risks for opioid-associated adverse effects, given their high prevalence, even though it is difficult to perform, often time consuming, and without any reliable evidence tools. Atluri et al (642) described the 3 cornerstones for responsible prescribing or stratifying patients by using screening tools into high, medium, and low-risk groups; monitoring patients by using urine drug screening, prescription monitoring programs, and pill counts; and lastly, establishing dose limits.

In risk stratification, it is important to utilize multiple models, incorporating psychological and behavioral factors to explain the pain experience $(12,110,200,281,300,567-602,646-650)$.

Based on the present evidence, regardless of the use of screening tools, patients may be classified into 3 categories as follows:

- Low risk - Low risk patients include those with a definable physical pathology; objective signs and reliable symptoms; clinical correlation with diagnostic testing including MRI, physical examination, and interventional diagnostic techniques; with or without mild psychological comorbidities; with or without mild coexisting medical disorders; no or well defined and controlled personal or family history of alcoholism or substance abuse; age of 45 or greater; high levels of pain acceptance and active coping strategies; and well-motivated patients with a willingness to participate in multimodal therapy and attempting to function at normal levels.

- Medium risk - Medium risk patients include those with significant pain problems with objective signs and symptoms confirmed by radiological evaluation, physical examination, or diagnostic interventions; with moderate psychological problems, 
well-controlled by medical therapy; moderate coexisting medical disorders well controlled by medical therapy and which are not affected by chronic opioid therapy such as central sleep apnea; those who develop mild tolerance but not hyperalgesia, without physical dependence or addiction; past history of personal or family history of alcoholism or substance abuse; involvement of more than 3 regions of the body; with defined pathology with moderate levels of pain acceptance and coping strategies; and willing to participate in multimodal therapy and attempting to function in their normal daily lives.

- High-risk - High-risk patients include those with widespread pain without objective signs and symptoms (involvement of more than 3 regions of the body); aberrant drug-related behavior; history of misuse, abuse, addiction, diversion, dependency, tolerance and hyperalgesia and alcoholism; with major psychological disorders; age of less than 45; HIV-related pain; high levels of pain exacerbation and low levels of coping strategies; unwilling to participate in multimodal therapy; and not functioning close to a near normal lifestyle.

The patients may be stratified into these categories with or without various tools, but with proper history, examination, and monitoring by PDMPs, UDT, and simple psychological evaluation.

\subsubsection{Recommendations}

Stratification of patients based on risk into high risk, medium risk, and low risk is crucial in initiation and maintenance of opioid therapy.

(Evidence: Level I-II; Strength of Recommendation: Moderate)

\subsection{Establishing Medical Necessity}

To establish medical necessity for opioid therapy, it is essential to have a physical diagnosis and information on inadequacy of multiple modalities of treatments including conservative, various other alternatives, and consultations if necessary. These include non-controlled substance therapy, physical modalities, behavioral interventions, interventional pain management techniques, and any other alternatives.

Medical necessity for opioids is established only when the following criteria are met: pain of moderate to severe degree, suspected organic problem, and documented failure to respond to non-controlled substance, adjuvant agents, physician ordered physical therapy, structured exercise program, and interventional techniques.

Opioids may be used as a second-line treatment. Appropriate documentation of psychological status must be documented. Further, medical necessity must be based on average, moderate, or severe ( $\geq 4$ on a scale of 0 to 10) pain and/or disability.

Behavioral interventions, interventional pain management, various other alternatives, and consultations as needed must be obtained.

\subsubsection{Recommendations}

It is essential to establish medical necessity prior to initiation or maintenance of opioid therapy based on average, moderate or severe ( $\geq 4$ on a scale of 0 to 10 ) pain and/or disability. (Evidence: Level II; Strength of Recommendation: Strong)

\subsection{Establishing Treatment Goals}

It is essential to establish treatment goals. Treatment goals should combine pain relief with improvement in activity and minimal or no adverse effects. To achieve the treatment goals, outcomes assessments are essential. Outcomes may be assessed by numeric rating scale pain ( 0 - 10 scale), functional assessment using the Oswestry Disability Index (0 - 50 scale), Neck Disability Index (0 - 50 scale), employment status, and/or improvement in activity status. The minimum amount of change in pain score in order to be clinically meaningful has been described as a 2-point change on a scale of 0 to 10 (or 20 percentage points), based on findings in trials which have been commonly utilized studying general chronic pain, chronic musculoskeletal pain, and chronic low back pain (12,651-656). Consequently, for guideline purposes, it would be appropriate to use clinically meaningful pain relief of at least $30 \%$ and/or a 3-point change on an 11-point scale of 0 - 10, or a clinically significant and/or functional status improvement of $30 \%$ or more. For interventional techniques, significant improvement has been defined as $50 \%$ reduction in pain scores and disability for evaluation purposes.

Before starting opioids, physicians should ensure that the patient's expectations are realistic. The goal of opioid therapy for chronic non-cancer pain is rarely the elimination of pain, but rather an improvement in function or a reduction of pain intensity. Before starting opioids, a discussion with the patient about specific goals related to pain reduction and functional improvement should address any unrealistic expectations. These 
goals, once established, should be documented in the patient's record; they are critical in determining that opioids are effective and should be monitored over time.

In establishing treatment goals, physicians should emphasize that there is insufficient evidence to determine long-term benefits of opioid therapy for chronic pain. In addition, physicians also should emphasize the increased risk for serious harms related to long-term opioid therapy that appears to be dose dependent (42). Treatment goals also should realize the differences between acute and chronic pain and associated expectations based on this understanding. While it is ideal to assess pain relief and functional status improvement, it may not be feasible routinely and specifically in diseases typically associated with progressive functional impairment or catastrophic injuries such as spinal cord trauma or chronic pancreatitis (42). Further, the goals of therapy must also include the information that if patients receiving opioid therapy for chronic pain do not experience meaningful improvement in both pain and function compared with prior to initiation of opioid therapy, tapering and discontinuation of opioid therapy should be considered as part of the agreement.

During the establishment of treatment goals, clinicians should also discuss with patients the known risks and realistic benefits of continuous opioid therapy. The goals should also emphasize the patient and clinician responsibilities for managing therapy.

\subsubsection{Recommendations}

It is essential to establish treatment goals of opioid therapy with regard to pain relief and improvement in function. (Evidence: Level I-II; Strength of Recommendation: Moderate)

\subsection{Assessment of Effectiveness of Opioid Therapy}

Multiple manuscripts, systematic and comprehensive reviews, and guidelines have been published evaluating the effectiveness and safety of opioids $(11,12,42,55,94,179-257)$. The clinical evidence based on RCTs shows insufficient evidence to determine long-term benefits of opioid therapy for chronic pain and shows an increased risk for serious adverse consequences related to long-term opioid therapy that appears to be dose dependent and also may be related to the combination of opioids with benzodiazepines and other drugs. However, the majority of the trials were of short-term duration. Consequently, there are no studies assessing the effectiveness of opioids on a long-term basis. However, the lack of randomized trials or even observational studies does not preclude the effectiveness of long-term opioid therapy. Chronic opioid therapy in appropriately selected patients may be beneficial. Thus, opioids provide effective pain control for a significant proportion of patients in combination with other therapies or in some patients as a stand alone treatment; however, they are not effective for all patients. Furthermore, as with other pharmacologic therapies, opioids are associated with multiple adverse consequences. Lower doses may also have similar, but less serious consequences.

Continued medical necessity depends on the following 4 "A's":

- Analgesia

- Activity

- Aberrant behavior

- Adverse effects

Improvement in pain relief and functional status must be assessed periodically and documented with at least improvement of $30 \%$ or greater, without adverse consequences.

Chronic opioid therapy in the elderly may be associated with multiple issues related to reduced hepatic and renal function, increased susceptibility to accumulation of opioids with a small therapeutic window, exacerbation of cognitive impairment, increased risk of medication errors, risk of falls, and finally, multiple comorbidities related to medical conditions and other drug therapies. Thus, it is essential to exercise additional caution in the elderly in providing chronic opioid therapy.

Patients with mental health conditions may require comorbid therapy with antianxiety medications as well as antidepressants. Every effort should be made to avoid concomitant use of benzodiazepines in the treatment of anxiety when combined with systemic opioids. Consideration of psychological conditions and treatment thereof may improve overall pain treatment outcomes; however, due to established risks with the combination of opioids and benzodiazepines and psychiatric instability including suicide risk, clinicians must cautiously provide chronic opioid therapy with or without benzodiazepines and antidepressant therapy, and also clinicians should consider behavioral health consultations, specifically in those with uncontrollable psychological disorders and suicide risk.

Pregnant women may be at increased risk of adverse consequences to both the mother and fetus. Some studies have shown stillbirth, poor fetal growth, 
pre-term delivery, birth defects, and, more importantly, neonatal opioid withdrawal syndrome in association with chronic opioid therapy. The effectiveness of opioid therapy in patients with a previous history of nonfatal overdoses has not been assessed. In patients with a nonfatal overdose, clinicians should carefully assess the risks, as well as educate and manage the patients with reduced opioid dosage, discontinuing opioids when possible.

The issue of chronic opioid therapy with long-acting opioids compared to short-acting opioids has been discussed with proponents and opponents using equally emotional arguments $(11,12,17,42,211,657,658)$. The present evidence shows the lack of superiority of longacting opioid therapy compared to short-acting opioid therapy $(11,12,42,211,657,658)$. However, long-acting opioids are associated with higher risk than short-acting opioids $(11,12,17,42,211,657,658)$. In fact, in 2014 , the FDA modified the labeling of extended release or long-acting opioid pain medications, noting serious risks, and recommending that these drugs be reserved for "management of pain severe enough to require daily, around-the-clock, long-term opioid treatment" $(659,660)$. Further, the FDA has recommended that long-acting opioids should not be used on a prn or as needed basis. The FDA has also noted that some longacting opioids are only appropriate for opioid-tolerant patients, defined as patients who have received $60 \mathrm{mg}$ equivalent dosages of oral morphine (MMEs) for at least one week (660). Dowell et al (42), in preparation of the CDC guidelines, were unable to find evidence that long-acting opioids were more effective or safer than intermittent use of immediate release opioids, or long-acting opioids reduced the risks for opioid misuse or addiction. Overall, long-acting opioid use can be associated with greater total average daily opioid dosage compared with short-acting opioids provided on an as needed basis.

Furthermore, there is no evidence for breakthrough pain in chronic pain or use of short-acting opioids in conjunction with long-acting opioids in chronic pain $(11,12,661)$. Opponents $(11,12,544,546,661)$ argue that immediate release opioids are typically offered several times a day, whereas long-acting opioids are offered once or twice a day. In addition, there may be multiple practical issues related to long-acting opioids with abuse deterrent formulations, and additional issues with reduced tolerability and the cost of the drug.

\subsubsection{Recommendations}

1. Clinicians must assess improvement based on analgesia, activity, aberrant behavior, and adverse effects and clinicians must document at least $30 \%$ improvement in pain or disability without adverse consequences. (Evidence: Level II; Strength of Recommendation: Moderate)

2. Clinicians must understand the effectiveness and adverse consequences of long-term opioid therapy in chronic non-cancer pain, as well as its limitations. (Evidence: Level I; Strength of Recommendation: Strong)

3. The evidence of effectiveness is similar for longacting and short-acting opioids with increased prevalence of adverse consequences of long-acting opioids. (Evidence: Level I-II; Strength of recommendation: Moderate to strong)

4. The long-acting opioids in high doses are recommended only in specific circumstances with severe intractable pain that is not amenable to short-acting opioids or moderate doses of longacting opioids, as there is no significant difference between long-acting and short-acting opioids for their effectiveness or adverse effects. (Evidence: Level I; Strength of Recommendation: Strong)

5. The evidence of effectiveness is similar for longacting and short-acting opioids, with increased prevalence of adverse consequences seen with long-acting opioids. (Evidence: Level I-II; Strength of recommendation: Moderate to strong)

\subsection{Dose Limits}

With overwhelming evidence for the misuse, abuse, and limited efficacy of chronic opioid therapy, the rationale for high-dose opioids continues to be weaker $(11,12,17,42,55,118,211,314,642-650,657,658,662-664)$. Generally, it is believed that patients who do not respond to a low or medium dose of opioids will not respond to larger doses, although individual circumstances also exist (642). In 2007, and then updated in 2010 (662), the state of Washington issued interagency guidelines that include guidance that the daily dose of opioids should not exceed $120 \mathrm{mg}$ of MME (662). The guidelines by APS and AAPM in 2009 defined "high doses" as $100 \mathrm{mg}$ MME (55). CDC guidelines (42) recommended a limit of $50-90 \mathrm{mg}$ MME. ASIPP guidelines (12) recommended a low dose as $40 \mathrm{MME}$. The Canadian Guidelines in 2010 identified 200 MME dose as a "watchful dose" (314). However, there has been only limited data verifying the safety of these recom- 
mended doses, especially in high-risk patients. Franklin et al (118) showed the effectiveness of dose limitation with reduction in dosage, frequency, and death rate. In addition, 5 studies showed that the rate of overdose was directly proportional to the prescribed opioid dose $(316,317,323)$. Bohnert et al $(317)$ concluded that the risk of opioid overdose increased when the opioid dose was equivalent to $50 \mathrm{MME}$ or higher. Dunn et al (316), in a population from a health maintenance organization (HMO) in Washington State, reported a 9-fold increase in opioid overdoses in patients receiving high dose opioids (> $100 \mathrm{MME}$ ) when compared to those getting low dose (< $20 \mathrm{MME}$ ). Paulozzi et al (323) found that, compared to patients receiving lower opioid doses or no opioid prescriptions, the risk of overdose was greater if daily opioid doses were above 40 MME. Gomes et al (663) found that patients receiving high doses (200 to $400 \mathrm{MME}$ ) and very high doses (> $400 \mathrm{MME}$ ) had a much higher overdose death rate than those getting moderate doses (<200 MME), with an overdose rate of 7.92 (9.94 per 1,000 population). Braden et al (664) showed that patients receiving more than 120 MME per day were more likely to have drug-related encounters than those getting lower doses. Franklin et al (118) showed that appropriate guidelines that considered $120 \mathrm{MME}$ as a high dose reduced overall opioids per day by $27 \%$ and long-acting Schedule II opioids by $37 \%$ in the proportion of the workers on doses of greater than $120 \mathrm{MME}$ per day. Moreover, the number of deaths was reduced by $50 \%$ from 2009 to 2010 . Rome et al (665), in a report of the outcomes at discharge of a chronic non-cancer pain rehabilitation program, showed that patients taking higher doses reported significantly greater catastrophizing and greater pain severity than the nonopioid group. Two other studies conducted in the worker's compensation population also showed similar results $(286,288)$. Adverse events were also reported more commonly at higher daily doses $(17,257,666)$.

Pascual et al (666) showed the increasing frequency of adverse effects of high dose tramadol (over $400 \mathrm{mg}$ ) compared with lower doses, with 2 patients experiencing seizures. Other studies $(257,327,667-669)$ have shown that there was a dose-dependent relationship between chronic opioid use, specifically with high doses and sleep disorders. Ballantyne and Mao (668), in 2003, indicated that doses higher than $100 \mathrm{MME}$ per day have not been validated in clinical trials and should be considered excessive.

The above evidence illustrates the dose-related effects at $40 \mathrm{MME}$ (323), $50 \mathrm{MME}(316,317), 120 \mathrm{MME}$
(670,671), and 200 MME (663). Thus far, it appears that of all the available literature correlates increasing mortality with increasing doses. In addition, several studies have demonstrated that for patients with severe pain on high opioid doses, tapering resulted in reduced pain and improved mood (314,665,671-673).

In contrast, among the remaining $20 \%$ of patients, $10 \%$ were prescribed high doses of opioids greater than 100 MME dose per day $(317,674,675)$ by single prescribers, accounting for an estimated $40 \%$ of the prescription opioid overdoses $(316,317)$. The remaining $10 \%$ of patients, seeing multiple doctors and typically involved in drug diversion, contributed to $40 \%$ of overdoses (169). Figure 16 shows the proportion of patients with drug overdoses, based on risk group (676).

Multiple studies in the literature have reported an association between opioid prescribing and overall health status, with increased disability, medical costs, and subsequent surgery, while on continued opioid use (73,91,281-292,677-686).

Therefore, we continued to recommend low-dose opioids up to $40 \mathrm{MME}$, moderate dose as 41 to $90 \mathrm{MME}$, and high dose as any dose after 91 or higher MME.

\subsubsection{Recommendation}

Opioid therapy must be started with short-acting opioids and should be maintained with low doses due to adverse consequences related to moderate to high dose opioid therapy.

(Evidence: Level II; Strength of Recommendation: Moderate)

\subsection{Informed Decision-Making}

Informed decision-making with appropriate consent is not only essential but mandatory. A discussion about potential benefits, adverse effects, complications, and risks helps the physician and the patient make a joint decision on whether to proceed with the opioid therapy $(12,42,454,687-689)$. There have been substantial descriptions in reference to informed consent and treatment agreements and their effectiveness or lack thereof $(12,42,454,687-699)$.

Before starting opioid therapy, appropriate realistic goals must be established. Patients should understand that opioid therapy will be discontinued if benefits do not outweigh risks. Clinicians should discuss all the risks associated with chronic opioid therapy including dependence, addiction, and death. The goals must be explicit and realistic, emphasizing the need for improvement in function despite pain. The risks of 


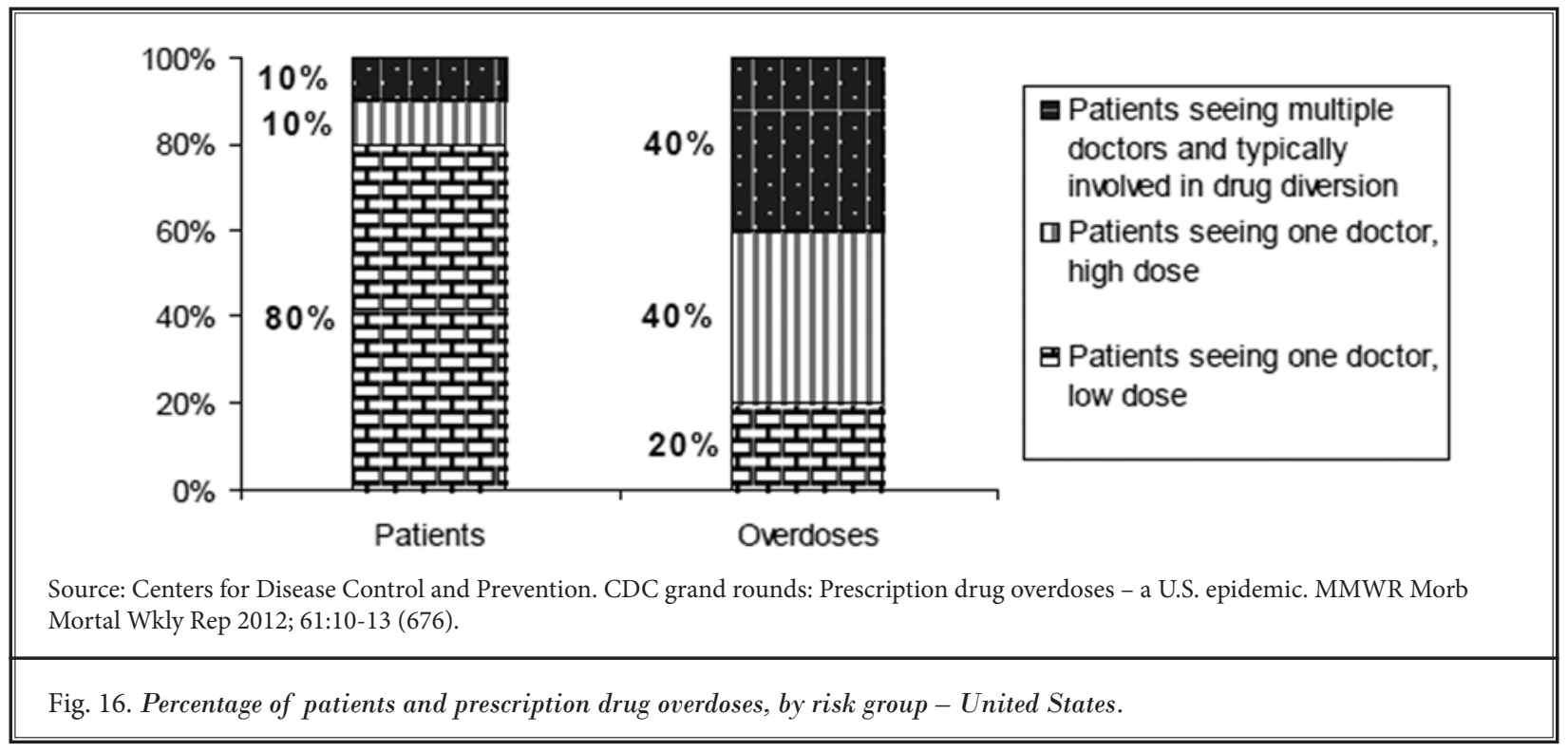

high dose opioid therapy and long-term opioid therapy must be emphasized. Appropriate adherence monitoring and periodic assessment of pain relief and function must be discussed.

The informed consent and treatment agreement should include clear descriptions of expectations regarding medication uses and abuses, as well as the consequences for violating the contract, which are as follows:

1) One prescribing doctor and one designated pharmacy

2) Urine/serum drug screening when requested

3) No early refills and no medications called in

4) If medications are lost or stolen, then a police report could be required before considering additional prescriptions.

Additional items to be included in an agreement are listed in Appendix I.

\subsubsection{Recommendations}

A robust agreement, which is followed by all parties, is essential prior to initiating and maintaining opioid therapy, as such agreements reduce overuse, misuse, abuse, and diversion. (Level of Evidence: Level III; Strength of Recommendation: Moderate)

\subsection{Initial Treatment}

Initiation of treatment is based on evaluation of the stratification of risk, knowledge and understanding of opioids, initiation with low-dose, short-acting, opioid therapy, and titration during an 8 to 12 week period.

\subsubsection{Initiation with Low-Dose Opioid Therapy}

A physician should follow the principles of prescribing as low an opioid dose as reasonably achievable or ALARA (as low as reasonably achievable), similar to radiation exposure guidelines, to provide therapeutic effect without major side effects (700).

Low dose therapy may be effective, with a reduction in the rate of complications, side effects, and adverse effects, specifically when opioid therapy is combined with other modalities, including interventional techniques $(12,17,42,208,283)$. Consideration of higher dosage requires careful reassessment of the pain and risk of misuse, and frequent monitoring with evidence of improved patient outcomes are necessary.

Thus, for moderate pain, first line therapy should start with tramadol, codeine, tapentadol, or hydrocodone. For second line mild to moderate pain therapy, clinicians should start with hydrocodone or oxycodone. For severe pain, first line therapy may start with hydrocodone, oxycodone, hydromorphone, or morphine, with second line therapy leading to fentanyl and if absolutely necessary, the third line therapy for severe pain with methadone or buprenorphine (314). The literature illustrates that codeine and tramadol may have a lower abuse risk than more potent opioids (314,701-703).

Methadone, though, has not been shown to be more effective than other opioids in most cases, and has been associated with multiple adverse consequences including death $(55,171,225,314,454,704-712)$. Methadone is also, however, dispensed in methadone clinics with very little regulation and supervision. Cli- 
nicians should follow FDA recommendations of limiting methadone to $30 \mathrm{mg}$ per day and only prescribe to patients nonresponsive to other opioids when it is absolutely necessary.

Meperidine is not recommended in chronic pain settings, due to adverse neurological events resulting in confusion and seizures with long-term treatment, secondary to the accumulation of the toxic metabolite normeperidine. The adverse events with meperidine are also increased with long-term use, renal insufficiency, and concurrent benzodiazepine use (713).

\subsubsection{Titrate}

Opioid medications must be started at low doses and titrated gradually to higher amounts if necessary. All attempts must be made to maintain patients on lower doses, including use of other drugs. Combinations of short- and long-acting opioids, and high doses of long-acting opioids, must be prescribed with extreme caution.

\subsubsection{Recommendations}

1. Once medical necessity is established, opioid therapy may be initiated with low doses and short-acting drugs, with appropriate monitoring to provide effective relief and avoid side effects. (Evidence: Level II; Strength of Recommendation: Moderate)

2. Consider up to $40 \mathrm{MME}$ as low dose, 41 to $90 \mathrm{MME}$ as moderate dose, and greater than $91 \mathrm{MME}$ as high dose.

(Evidence: Level II; Strength of Recommendation: Moderate)

3. Long-acting opioids should not be utilized for the initiation of opioid therapy. (Evidence: Level l; Strength of Recommendation: Strong)

4. Methadone is recommended for use after failure of other opioid therapy and only by clinicians with specific training in its risks and uses. (Evidence: Level I; Strength of Recommendation: Strong)

\subsection{Adherence Monitoring}

The role of adherence monitoring with various tools has been described as part of the initial evaluation. This must be continued through the treatment phase, using PDMPs, UDT, pill counts, and behavioral assessment during each visit. Adherence monitoring is dependent on risk stratification. Monitoring based on risk stratification is illustrated in Fig. 17 (642). An algorithmic approach to UDT is illustrated in Fig. 18 (457).
However, regulations with stricter criteria take priority over these algorithmic approaches.

Aberrant drug-related behaviors include alteration of prescriptions or the route of delivery, doctor shopping or accessing opioids from other sources, multiple unauthorized dose escalations, drug seeking behavior with focus on certain types of opioids and benzodiazepines, loss of prescriptions, requests for early refills, aggressive complaining, staff harassment, complaining about other patients, questioning rights and responsibilities, repeated withdrawal symptoms, exacerbation of underlying mood or anxiety disorders, alcohol use, poor social functioning, loss of job and loss of activities of daily living, and emphatic views on opioid medication and illicit drugs as well as legalization of illicit drugs.

\subsubsection{Recommendations}

1. Monitoring recommendation for methadone prescription is that an electrocardiogram should be obtained prior to initiation, at 30 days, with dose adjustments, concomitant medications that may affect QTC interval, and yearly thereafter. Evidence: Level I; Strength of Recommendation: Strong)

2. In order to reduce prescription drug abuse and doctor shopping, adherence monitoring by UDT and PDMPs provide evidence that is essential to the identification of those patients who are non-compliant or abusing prescription drugs or illicit drugs. (Evidence: Level I-Il; Strength of Recommendation: Moderate to strong)

\subsubsection{Monitoring and Managing Side Effects}

Multiple side effects, including effect on driving, sedation, constipation, and breathing (specifically in patients with respiratory disorders), must be monitored.

Constipation is one of the most common opioidrelated adverse effects (302) and may become a major issue in a significant proportion of patients with continued exposure to opioids. In addition, in older adults or other patients with additional reasons to develop constipation, constipation may be more frequent and also problematic. Consequently, a physician should consider the initiation of a bowel regimen even before the development of constipation and definitely after the development of constipation. Even though the evidence for bowel regimen is anecdotal, the use of increased fluid and fiber intake, stool softeners, and laxatives are often simple and effective. Multiple publications have evaluated opioid antagonists in the prevention or treatment of opioid-induced bowel dysfunction $(195,714)$, but the 


\section{Chronic Pain}

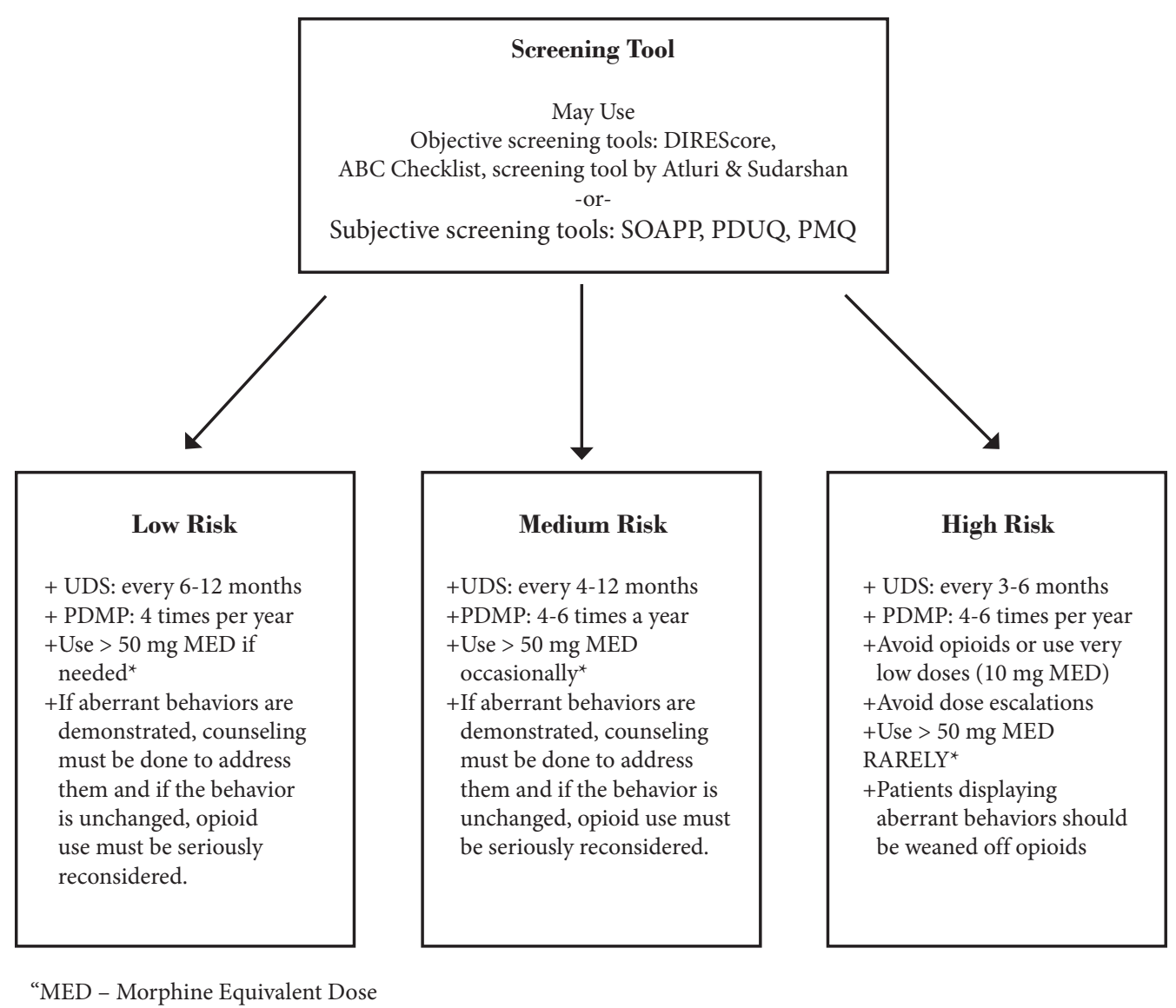

Reproduced with permission from: Atluri SL, Akbik H, Sudarshan G. Prevention of opioid abuse in chronic non-cancer pain: An algorithmic, evidence-based approach. Pain Physician 2012; 15:ES177-ES189 (642).

Fig. 17. Risk stratification and adherence monitoring.

evidence is insufficient to recommend such antagonists to prevent bowel dysfunction.

During dosage titration in a trial of opioid therapy, advise the patient to avoid engaging in dangerous activities, such as driving a motor vehicle or the use of heavy machinery, until a stable dosage is established and it is certain that the opioid dose does not cause sedation, as well as when taking opioids with alcohol, benzodiazepines, or other sedating drugs (314). When assessing safety to drive in patients on long-term opioid therapy, consider factors that could impair cognition and psychomotor ability, such as a consistently severe pain rating, disordered sleep, and concomitant medications that increase sedation (314).

\subsubsection{Recommendations}

1. It is essential to monitor for side effects and manage them appropriately, including discontinuation of opioids if indicated. (Evidence: Level l; Strength of Recommendation: Strong)

2. Constipation must be closely monitored, and a bowel regimen should be initiated as soon as deemed necessary. (Evidence: Level I; Strength of Recommendation: Strong) 
Responsible, Safe, and Effective Prescription of Opioids for Chronic Non-Cancer Pain

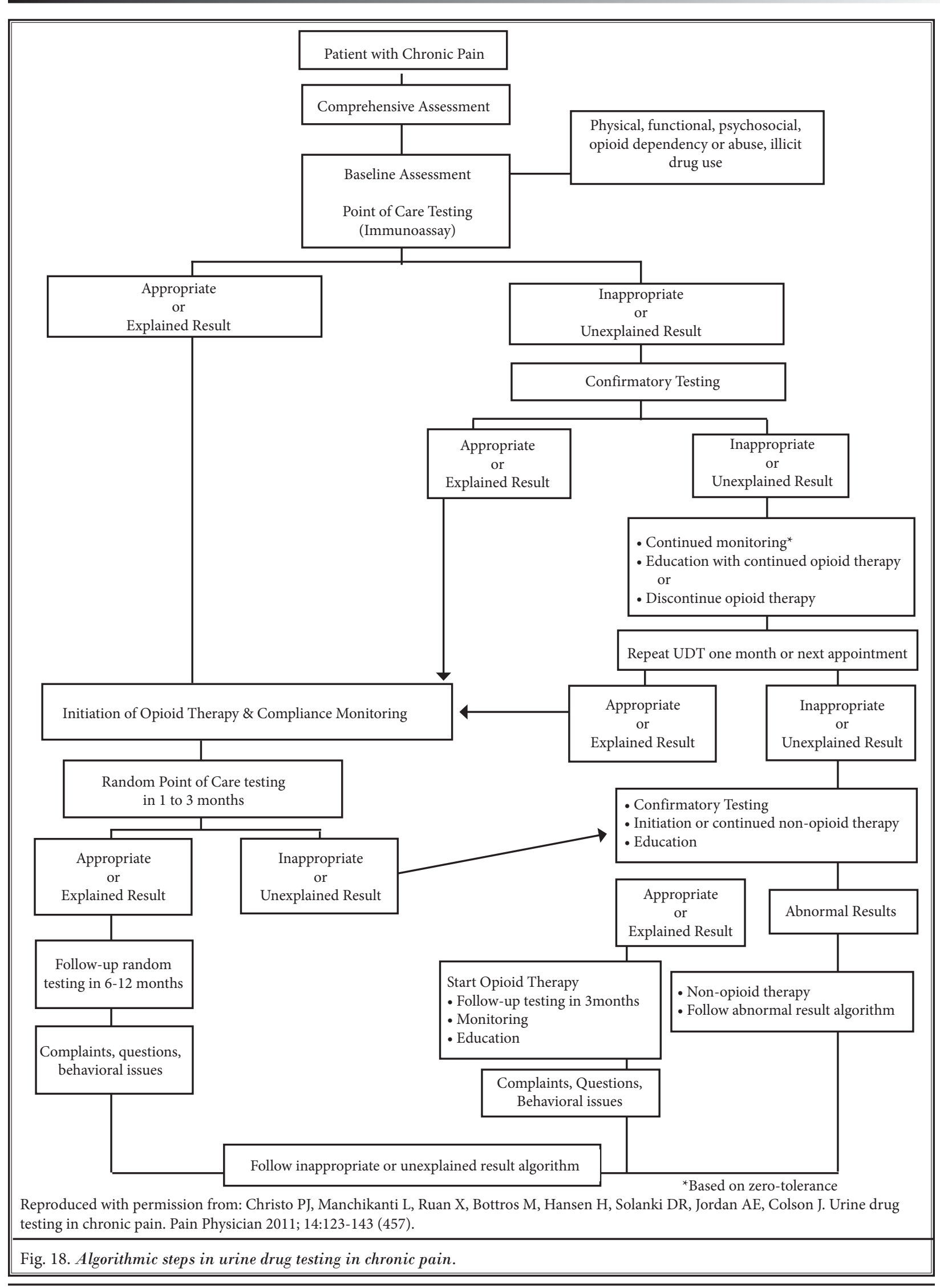


3. It is recommended that a policy of driving under the influence of drugs be developed and monitored during initiation of therapy, changes in the dosages, and addition of other centrally acting agents. (Level of Evidence: Level III; Strength of Recommendation: Moderate)

\subsection{The Final Phase}

After initiation of opioid therapy and stable maintenance for 8 to 12 weeks with appropriate outcomes, it is essential to arrive at a conclusion to either continue or to discontinue the opioids.

If the patient continues with persistent pain or there is new pain, a comprehensive evaluation must be repeated or a referral may be made. Similarly, if there is any indication of abuse, misuse, lack of analgesia, lack of activity, adverse effects, or aberrant behavior, the physician must taper the drug therapy and discontinue. Alternate modalities must be pursued at this stage.

Opioid therapy is continued if appropriate analgesia and functional status is achieved, either with opioid therapy alone or in conjunction with other modalities. Minimal requirements for continued opioid therapy are analgesia of at least $30 \%$, and/or activity improvement of $30 \%$ without misuse/abuse, or major adverse effects. However, if treatment is successful, one may attempt to wean from opioids. If necessary to continue, monitoring must be continued and the patient be discharged either with improvement or with any deficiencies.

In patients with dependency, office-based opioid dependence treatment may be provided. In a narrative review, Colson et al (715) described that office-based opioid dependence treatment is a viable alternative to methadone treatment or rehabilitation programs. However, office-based treatment of opioid dependency requires a special licensure from the DEA. Thus, for physicians providing opioid management of pain, the use of buprenorphine/naloxone is an important tool to consider for opioid dependence issues which arise when treating chronic pain.

If it is required, tapering or discontinuation of opioid therapy may be considered; however, for a patient who has not been taking medication on a long-term basis, tapering or weaning is not necessary and discontinuation may be carried out. Tapering may be carried out slowly with a decrease by $10 \%$ of the original dose per week. This is generally well tolerated with minimal adverse physiological effects. However, some patients can be tapered or weaned more rapidly without any major problems over a 6 to 8 week period. During this period, if opioid abstinence syndrome is encountered, it is rarely medically serious, even though symptoms may be quite unpleasant. The symptoms of abstinence syndrome, including nausea, diarrhea, muscle pain, and myoclonus, can be managed with clonidine 0.1 to 0.2 mg orally every 6 hours or clonidine transdermal patch $0.1 \mathrm{mg} 24$ hours weekly during the taper. Patients should be monitored often for significant hypotension and anticholinergic side effects. While rare, in some patients it may be necessary to slow the tapering and weaning timeline from weekly to monthly dosage adjustments. If the patient is not following the tapering dosages or abusing them, then tapering is going to be unsuccessful and patients must be referred to detoxification facilities.

Symptoms of mild opioid withdrawal occasionally persist for 6 months after opioids have been discontinued. The physician may consider using adjuvant agents such as antidepressants to manage irritability and sleep disturbance or antiepileptics for neuropathic pain. However, physicians should be cautious and preferably not treat withdrawal symptoms with opioids or benzodiazepines once the weaning process or discontinuation of opioids is started. The patient may be referred for counseling or other support during the weaning period if there are significant behavioral issues. If such issues arise, the physician should refer the patient to a chemical dependency center for complicated withdrawal symptoms. Physicians not trained in pain management may refer their patients with these issues to pain management specialists or addictionologists.

\subsubsection{Recommendations}

1. Chronic opioid therapy may be continued, with continuous adherence monitoring, and modified at any time during this phase, in conjunction with or after failure of other modalities of treatments with improvement in physical and functional status and minimal adverse effects. (Evidence: II-III; Strength of Recommendation: Moderate)

2. Chronic opioid therapy should be monitored for adverse effects, and those side effects should be managed appropriately. (Evidence: I; Strength of Recommendation: Strong)

\subsection{Documentation}

The physician should keep accurate and complete medical records, which include all aspects of interventional pain management and medical care. These comprise, but are not limited to: 
- Medical history and physical examination

- Diagnostic, therapeutic, and laboratory results

- Evaluations and consultations

- Treatment objectives

- Discussion of risks, benefits, and limitations of treatments

- Details of different treatments and medications, including date, type, dosage, and quantity prescribed

- Instructions to the patient

- Periodic reviews of outcomes, including documentation of functional status, preferably using validated tools.

Records should remain current and be maintained in an accessible manner and readily available for review, not only for the physician and other members of the practice, but also for authorities.

To be in compliance with controlled substance laws and regulations required to prescribe, dispense, or administer controlled substances, the physician must have an active license in the state and comply with applicable federal and state regulations. Various licensure boards have published regulations and recommendations for prescribing controlled substances. Physicians are advised to refer to those regulations for their respective state. Physicians should not prescribe scheduled drugs for themselves or immediate family except in emergency situations.

The following criteria should be considered carefully in providing controlled substances:

1. Complete initial evaluation, including history and physical examination

2. Psychological evaluation

3. Physiological and functional assessment, as necessary and feasible

4. Indications and medical necessity

5. The use of the lowest possible dose to provide adequate analgesia with minimum side effects should be the goal of opioid therapy

6. In general, do not combine opioids with sedativehypnotics, benzodiazepines, or barbiturates for chronic, non-cancer pain unless there is a specific medical indication for the combination

7. Adherence to the controlled substance agreement with patients understanding the risks and benefits of controlled substances and the policy and regulations of the practitioner, including controlled substances being prescribed by only one practitioner and being obtained from only one pharmacy

8. Advise patient to contact the prescribing clinician with any acute illness so that a consideration can be given to temporary dose reduction while patient is ill

9. Monitoring for drug abuse or diversion should be routine, and if confirmed, referral to rehabilitation centers may be made, with termination of prescriptions of controlled substances.

\subsection{Summary of Steps for Chronic Opioid Therapy}

The evidence synthesis and guidance preparation provides the following recommendations with 10 steps to opioid therapy:

\subsection{Initial Steps of Opioid Therapy}

1. Comprehensive assessment and documentation is recommended before initiating opioid therapy. This includes documentation of comprehensive history, general medical condition, psychosocial history, psychiatric status, and substance use history. (Evidence: Level I; Strength of Recommendation: Strong)

2. Screening for opioid abuse is recommended to potentially identify opioid abusers and reduce opioid abuse. (Evidence: Level II-III; Strength of Recommendation: Moderate)

3. PDMPs must be implemented. PDMPs provide data on patterns of prescription usage, potentially reducing prescription drug abuse or doctor shopping and may reduce emergency room visits, drug overdoses, or deaths. (Evidence: Level I-II; Strength of Recommendation: Moderate to strong)

4. UDT must be implemented at initiation of opioid therapy, along with continued adherence monitoring to identify patients who are non-compliant or abusing prescription drugs or illicit drugs. UDT may decrease prescription drug abuse or illicit drug use in patients on chronic pain management therapy. (Evidence: Level II; Strength of Recommendation: Moderate)

5. Establish appropriate physical diagnosis and psychological diagnosis if available prior to initiating opioid therapy. (Evidence: Level I; Strength of Recommendation: Strong)

6. Appropriate imaging, physical diagnosis, and psychological status should be considered before establishing opioid therapy. These findings should be coordinated with subjective complaints. (Evidence: Level II; Strength of Recommendation: Moderate)

7. It is essential to establish medical necessity prior to initiation or maintenance of opioid therapy. (Evidence: Level I; Strength of Recommendation: Strong) 
8. Stratify patients based on risk. (Evidence: Level I-II; Strength of Recommendation: Moderate)

9. It is essential to establish treatment goals of opioid therapy with regard to pain relief and improvement in function. (Evidence: Level I-Il; Strength of Recommendation: Moderate)

\subsection{Assessment of Effectiveness of Opioid Therapy}

10. A robust opioids agreement, which is followed by all parties, is essential prior to initiating and maintaining opioid therapy, as such agreements reduce overuse, misuse, abuse, and diversion. (Evidence: Level III; Strength of Recommendation: Moderate)

11. Once medical necessity is established, opioid therapy may be initiated with low doses and short-acting drugs with appropriate monitoring to provide effective relief and avoid side effects. (Evidence: Level II; Strength of Recommendation: Moderate)

12. Consider up to $40 \mathrm{MME}$ as low dose, 41 to $90 \mathrm{MME}$ as a moderate dose, and greater than $91 \mathrm{MME}$ as high doses. (Evidence: Level II; Strength of Recommendation: Moderate)

13. Long-acting opioids should not be utilized for the initiation of opioid therapy. (Evidence: Level l; Strength of Recommendation: Strong)

14. Methadone is recommended only for use after failure of other opioid therapy and only by clinicians with specific training in its risks and uses within FDA recommended doses. (Evidence: Level l; Strength of Recommendation: Strong)

15. Clinicians must understand and educate patients on the effectiveness and adverse consequences of long-term opioid therapy in chronic non-cancer pain as well as its limitations. (Evidence: Level I; Strength of Recommendation: Strong)

16. The evidence of effectiveness is similar for longacting and short-acting opioids with increased prevalence of adverse consequences with longacting opioids. (Evidence: Level I-II; Strength of recommendation: Moderate to strong)

17. Periodically assess pain relief and/or functional status improvement of $\geq 30 \%$ without adverse consequences. (Evidence: Level II; Strength of recommendation: Moderate)

18. Long-acting or high dose opioids are recommended only in specific circumstances with severe intractable pain that is not amenable to shortacting opioids, as there is no significant difference between long-acting and short-acting opioids for their effectiveness or adverse effects. (Evidence: Level I; Strength of Recommendation: Strong)

\subsection{Monitoring for Adherence and Side Effects}

19. In order to reduce prescription drug abuse and doctor shopping, adherence monitoring by UDT and PDMPs provide evidence that is essential to the identification of those patients who are non-compliant or abusing prescription drugs or illicit drugs. (Evidence: Level I-Il; Strength of Recommendation: Moderate to strong)

20. Monitoring recommendation for methadone prescription is that an electrocardiogram should be obtained prior to initiation, at 30 days, after increases in dose, and yearly thereafter. (Evidence: Level I; Strength of Recommendation: Strong)

21. Monitor for side effects including constipation and manage them appropriately including discontinuation of opioids when indicated. (Evidence: Level l; Strength of Recommendation: Strong)

\subsection{The Final Phase}

22. Chronic opioid therapy may be continued, with continuous adherence monitoring, and modified at any time during this phase, in well-selected populations, in conjunction with or after failure of other modalities of treatments with improvement in physical and functional status and minimal adverse effects, with appropriate outcomes. (Evidence: Level I-II; Strength of Recommendation: Moderate)

23. Discontinue opioid therapy for lack of response, adverse consequences, and abuse with rehabilitation. (Evidence: Level III; Strength of Recommendation: Moderate)

\subsection{Conclusion}

These guidelines were developed based on a comprehensive review of the literature, consensus among the panelists, and practice patterns. There have been many fatalities associated with the increasing therapeutic use and abuse of opioids. These unwanted effects may be related to a lack of understanding and education in the proper application of opioid therapy, but more importantly, lack of availability of current overdose data, lack of unique codes for drugs such as fentanyl with escalating cause of overdoses, and hindering efforts to explore the potential source of opioids 
that can be licitly prescribed or illicitly manufactured. The opioid overdose epidemic no longer appears to be based on prescriptions alone as the accelerating pace of the opioid epidemic continues while opioid prescribing by physicians has been significantly reduced. The evidence supporting the efficacy for use of opioids as a treatment for chronic non-cancer pain is fair for shortterm with improvement in pain and function, whereas it is limited, with an absence of evidence regarding the long-term efficacy or effectiveness. However, patientbased surveys and physician surveys provide contradictory results with significant improvement in patients with appropriate medical necessity and indication. Even though physical and functional status improvement appear to be lacking in the patients with opioid therapy, recent surveys $(33,34,544,545,547-549)$ have shown that $70 \%$ of the long-term opioid users suffer with a chronic disability, handicap, or chronic disease which keeps them from participating fully in work, school, housework, or other activities (34). Consequently, existing need and emerging evidence shows at least perceived need for opioid therapy, when medically necessary. However, existing need and emerging evidence shows the need for opioid therapy in patients with proven medical necessity and stability with improvement in pain and function, independently or in conjunction with other modalities of treatments in low doses.

For practitioners considering opioid use, multiple recommendations for opioid management are sum- marized. The majority of treatment recommendations are based on evidence consensus and practice patterns, rather than high quality evidence alone. Thus, opioids for chronic non-cancer pain should be reserved for select patients with moderate or severe pain that significantly affects function or QOL. Appropriate evaluation, documentation, screening, and risk stratification is indicated from initiation through the continuation of opioid therapy.

In conclusion, the focus of these updated guidelines continues to be to objectively integrate both the evidence and consensus and practice patterns with the goal of curbing opioid abuse, misuse, and overuse, and at the same time maintain access to opioids for patients who are in need of them.

\section{Acknowledgments}

The authors wish to thank Laurie Swick, BS, for manuscript review; and Tonie M. Hatton and Diane E. Neihoff, transcriptionists, for their assistance in preparation of this manuscript. We would like to thank the editorial board of Pain Physician for review and criticism in improving the manuscript.

\section{Disclosures}

Funding: There was no external funding in the preparation of this manuscript. Internal funding provided by the American Society of Interventional Pain Physicians (ASIPP) was limited to travel and lodging expenses of the authors.

\section{Author Affiliations}

\section{Laxmaiah Manchikanti, MD}

Dr. Manchikanti is Medical Director of the Pain Management Center of Paducah, Paducah, KY, and Clinical Professor, Anesthesiology and Perioperative Medicine, University of Louisville, Louisville, KY.

drlm@thepainmd.com

Adam M. Kaye, PharmD

Dr. A. M. Kaye is Clinical Professor of Pharmacy, Thomas J. Long School of Pharmacy and Health Sciences, University of the Pacific, Stockton, CA.

akaye@pacific.edu

Nebojsa Nick Knezevic, MD, PhD

Dr. Knezevic is Vice Chair for Research and Education, Advocate Illinois Masonic Medical Center, and Clinical Associate Professor of Anesthesiology and Surgery, University of Illinois, Chicago, IL. nick.knezevic@gmail.com
Heath McAnally, MD

Dr. McAnally is Medical Director, Northern Anesthesia \& Pain Medicine, LLC, Eagle River, AK.

northernpainmedicine@gmail.com

Andrea M. Trescot, MD

Dr. Trescot is the Director, The Pain \& Headache Center, Wasilla, AK.

drtrescot@gmail.com

Susan Blank, MD

Dr. Blank is Founder and Chief Medical Officer of Atlanta Healing Center Atlanta Healing Center, LLC, Norcross, GA. susanblankmd@hotmail.com

\section{Vidyasagar Pampati, MSc}

Vidyasagar Pampati is a Statistician at the Pain Management Center of Paducah, Paducah, KY.

sagar@thepainmd.com 
Salahadin Abdi, MD, PhD

Dr. Abdi is Professor and Chair, Department of Pain Medicine, University of Texas, MD Anderson Cancer Center, Houston, TX. sabdi@mdanderson.org

\section{Jay S. Grider, DO, PhD}

Dr. Grider is Associate Chief Medical Officer of Ambulatory Services, Medical Director of UKHealthCare Pain Services, and Professor of the Department of Anesthesiology, University of Kentucky College of Medicine, Lexington, KY. jsgrid2@email.uky.edu

\section{Alan D. Kaye, MD, PhD}

Dr. Kaye is Professor and Chair, Department of Anesthesia and Professor, Department of Pharmacology, LSU Health Science Center, New Orleans, LA.

akaye@lsuhsc.edu

Kavita N. Manchikanti, MD

Dr. Kavita Manchikanti is an interventional pain physician at Pain Management Center of Paducah, Paducah, KY.

drkavita@thepainmd.com

\section{Harold J. Cordner, MD}

Dr. Cordner is with Florida Pain Management Associates, Sebastian, FL; and Associate Clinical Professor Florida State University College of Medicine, Tallahassee, FL. gassdoc@aol.com

\section{Christopher G. Gharibo, MD}

Dr. Gharibo is Medical Director of Pain Medicine and Associate Professor of Anesthesiology and Orthopedics, Department of Anesthesiology, NYU Langone-Hospital for Joint Diseases, NYU School of Medicine, New York, NY.

cgharibo@usa.net

\section{Michael E. Harned, MD}

Dr. Harned is Associate Professor with the Department of Anesthesiology, Division of Pain Medicine, and Division Chief of Interventional Pain Medicine at the University of Kentucky College of Medicine, Lexington, KY.

meharn2@email.uky.edu

\section{Sheri L. Albers, MD}

Dr. Albers is Director of Research, Radiology Research and Consultation, Sacramento, CA.

Sla2oz@aol.com

\section{Sairam Atluri, MD}

Dr. Atluri is Medical Director, Tri-State Spine Care Institute, Cincinnati, $\mathrm{OH}$.

saiatluri@gmail.com

\section{Steve M. Aydin, DO}

Dr. Aydin, Specialist in Physical Medicine and Rehabilitation and Interventional Pain Management, Manhattan Spine and Pain Medicine, and Hofstra-North Shore/LIJ School of Medicine, New York, NY.

steve.aydin@gmail.com

\section{Sanjay Bakshi, MD}

Dr. Bakshi is President of Manhattan Spine and Pain Medicine, New York, NY; and Director of Pain Management, Lenox Hill Hospital, New York, NY.

drbakshi58@gmail.com

\section{Robert Barkin, MBA, PharmD}

Dr. Barkin is Professor Rush Medical College: Anesthesiology, Family Medicine, and Pharmacology; Clinical Pharmacologist, Department Anesthesiology, Pain Centers, NorthShore University HealthSystem Evanston, IL, and Skokie Hospitals, Skokie, IL

rbarkin@rush.edu

\section{Ramsin M. Benyamin, MD}

Dr. Benyamin is Medical Director, Millennium Pain Center, Bloomington, IL, and Clinical Assistant Professor of Surgery, College of Medicine, University of Illinois, Urbana-Champaign, IL.

ramsinbenyamin@yahoo.com

\section{Mark V. Boswell, MD, PhD}

Dr. Boswell is Professor and Chair, Department of Anesthesiology and Perioperative Medicine, University of Louisville, Louisville, KY

boswellmv@earthlink.net; ; mark.boswell@louisville.edu

\section{Ricardo M. Buenaventura, MD}

Dr. Buenaventura is Medical Director, Pain Relief of Dayton, Centerville, $\mathrm{OH}$, and Clinical Associate Professor, Department of Surgery, Wright State University School of Medicine, Dayton, $\mathrm{OH}$.

rbuena@yahoo.com

\section{Aaron K. Calodney, MD}

Dr. Calodney is Director of Clinical Research, Precision Spine Care, Texas Spine and Joint Hospital, Tyler, TX.

aaroncalodney@me.com

\section{David L. Cedeno, PhD}

Dr. Cedeño is the Director of Basic Research at the Millennium Pain Center, Bloomington, IL, and an Associate Professor of Chemistry at Illinois State University, Normal, IL.

dcedeno@millenniumpaincenter.com

\section{Sukdeb Datta, MD}

Dr. Datta is Medical Director, Datta Endoscopic Back Surgery and Pain Center and Professorial Lecturer, Department of Anesthesiology, Mount Sinai School of Medicine, New York, NY.

sukdeb@hotmail.com; sdattamd@gmail.com

\section{Timothy R. Deer, MD}

Dr. Deer is President and CEO of The Center for Pain Relief, Charleston, WV, and Clinical Professor of Anesthesiology and Pain Medicine, West Virginia University School of Medicine, Charleston, WV.

DocTDeer@aol.com 


\section{Bert Fellows, MA}

Bert Fellows is Director Emeritus of Psychological Services at the Pain Management Center of Paducah, Paducah, KY.

Bert06@embargmail.com

\section{Vincent Galan, MD}

Dr. Galan is CEO and Medical Director, Georgia Pain Care, Atlanta, GA.

vgalan@msn.com

\section{Vahid Grami, MD, MPH}

Dr. Grami is an Associate Physician in Pain Medicine and Anesthesiology at Geisinger Medical Center Interventional Pain Center Woodbine, Danville, PA.

vgrami1@geisinger.edu

\section{Hans Hansen, MD}

Dr. Hansen is Medical Director, Pain Relief Centers, Conover, NC. hhansen@painreliefcenters.com

\section{Standiford Helm II, MD}

Dr. Helm is Medical Director, The Helm Center for Pain Management, Laguna Woods, CA.

drhelm@thehelmcenter.com

\section{Rafael Justiz, MD}

Dr. Justiz is President and CEO of the Oklahoma Pain Physicians, and Clinical Associate Professor of Anesthesiology and Pain Medicine University of Oklahoma Health Sciences Center, Oklahoma City, OK.

rjustiz@yahoo.com

\section{Dhanalakshmi Koyyalagunta, MD}

Dr. Koyyalagunta is Associate Professor, Medical Director, Pain Management Center, University of Texas, MD Anderson Cancer Center, Dept. of Anesthesiology \& Pain Medicine, Houston, TX dkoyyala@mdanderson.org

\section{Yogesh Malla, MD}

Dr. Malla is an Interventional Pain Physician at the Pain Management Center of Paducah, Paducah, KY.

Yogesh@thepainmd.com

\section{Annu Navani, MD}

Dr. Navani is Medical Director, Comprehensive Spine \& Sports Center, Campbell, CA, and Adjunct Clinical Associate Professor, Stanford University School of Medicine, Stanford, CA. anavani@spineandsportsctr.com

\section{Kent Nouri, MD}

Dr. Nouri is with the Department of Pain Medicine, University of Texas MD Anderson Cancer Center, Houston, TX KHNouri@mdanderson.org

\section{Ramarao Pasupuleti, MD}

Dr. Pasupuleti is Medical Director, Center for Pain Management, Bowling Green, KY. rampasupuleti@yahoo.com

\section{Nalini Sehgal, MD}

Dr. Sehgal is Director, Interventional Pain Program, and Professor and Director Pain Fellowship, Department of Orthopedics and Rehabilitation Medicine, University of Wisconsin School of Medicine and Public Health, Madison, WI.

Sehgal@rehab.wisc.edu

\section{Sanford M. Silverman, MD}

Dr. Silverman is Medical Director of Comprehensive Pain Medicine, Pompano Beach, FL.

sanfordsilverman@cpmedicine.com

Thomas T. Simopoulos, MD

Dr. Simopoulos is Assistant Professor, Department of Anesthesia, Critical Care and Pain Medicine, Beth Israel Deaconess Medical Center, Harvard Medical School, Boston, MA.

tsimopou@bidmc.harvard.edu

Vijay Singh, MD

Dr. Singh is Medical Director, Spine Pain Diagnostics Associates, Niagara, WI

vj@wmpnet.net

Konstantin V. Slavin, MD

Dr. Slavin is Professor and Section Head, Stereotactic and Functional Neurosurgery, College of Medicine, University of Illinois at Chicago, Chicago, IL.

kslavin@uic.edu

Daneshvari R. Solanki, MD

Dr. Solanki is Professor of Anesthesia and Pain Management, University of Texas Medical Branch, Galveston, TX. dsolanki@utmb.edu

\section{Peter S. Staats, MD}

Dr. Staats is Managing Partner of Premier Pain Centers, Adjunct Associate Professor, Department of Anesthesiology and Critical Care Medicine, Shrewsbury, NJ, and the Department of Oncology at Johns Hopkins University School of Medicine, Baltimore, MD.

peterstaats@hotmail.com

\section{Ricardo Vallejo, $\mathrm{MD}, \mathrm{PhD}$}

Dr. Vallejo is Director of Research, Millennium Pain Center, Bloomington, IL; and Adjunct Research Professor, Illinois Wesleyan University, Bloomington, IL.

RVallejo@millenniumpaincenter.com

\section{Bradley W. Wargo, DO}

Dr. Wargo is an Interventional Pain Physician at the McFarland Clinic, Mary Greeley Medical Center, Ames, IA. drbwargo@gmail.com

\section{Art Watanabe, MD}

Dr. Watanabe is an Interventional Pain Physician, Mt Baker Pain Center, Bellingham, WA.

aswatanabe@earthlink.net 
Joshua A. Hirsch, MD

Dr. Hirsch is Vice Chief of Interventional Care, Chief of NeuroInterventional Spine, Service Line Chief of Interventional Radiology, Director Interventional and Endovascular Neuroradiology, Massachusetts General Hospital; Harvard Medical School, Boston, MA.

hirsch@snisonline.org

\section{Conflicts of Interest}

\section{Laxmaiah Manchikanti, MD}

Dr. Manchikanti has provided limited consulting services to Semnur Pharmaceuticals, Incorporated, which is developing nonparticulate steroids.

\section{Steve M. Aydin, DO}

Dr. Aydin is a consultant and receives payment for development of educational presentations for Pfizer and Horizon.

\section{Robert Barkin, MBA, PharmD}

Dr. Barkin is a speaker for Astra Zeneca, Endo, DepoMed, and Collegium.

\section{Ramsin M. Benyamin, MD}

Dr. Benyamin is a consultant and lecturer for Boston Scientific and Kimberly Clark.

\section{Aaron K. Calodney, MD}

Dr. Calodney is a consultant for Medtronic, SI-Bone, Stryker, Nevro, and APEX Biologix.

\section{Harold J. Cordner, MD}

Dr. Cordner has provided expert testimony for the Florida Department of Health, and has received travel/accommodations expenses covered or reimbursed by NANS.

\section{Timothy R. Deer, MD}

Dr. Deer is a consultant for Axonics, Bioness, Abbott, Nevro, Jazz Pharm, SpineThera, Saluda, Medtronic, Vertos, Flowonix, and Ethos; minor stock/stock options with Axonics, Bioness, SpineThera, Saluda, Vertos, and Ethos; travel/accommodations expenses covered or reimburse by Abbott and Axonics; and has funded research with Saluda, Nevro, Mainstay, Abbott, Medtronic, Bioness, and JazzPharm.

\section{Vincent Galan, MD}

Dr. Galan is a consultant for Medtronic; on the advisory board for Vertiflex (Totalis); and has received research grants from Nevro Corp, Medtronic, and St. Jude Medical.

\section{Christopher G. Gharibo, MD}

Dr. Gharibo receives payment for development of education presentations, as well as travel/accommodations expenses from Pernix, Mallinckrodt, Daichii Sankyo, Colegium, Astra Zenecam, Depomed, Iroko, TEVA, Kaleo, Endo, Shionogi, Nuven, and Axxsome.
Jay S. Grider, DO, PhD

Dr. Grider is a consultant for Medtronic and St. Jude and is an Interlink Spine medical advisory board shareholder.

\section{Hans Hansen, MD}

Dr. Hansen receives payment for development of educational presentations from Depomed, stock/stock options from Phizer, and travel/accommodation expenses from Endo Pharmaceuticals and Speakers Bureaus.

\section{Michael E. Harned, MD}

Dr. Harned receives payment for development of educational presentations from Medtronic and honorarium from St. Jude Medical.

\section{Standiford Helm II, MD}

Dr. Helm is a clinical investigator with Epimed and receives research support from Cephalon/Teva, AstraZeneca, and Purdue Pharma, LP. He has attended an advisory group meeting for Activas.

\section{Joshua A. Hirsch, MD}

Dr. Hirsch is a consultant for Medtronic.

\section{Rajael Justiz, MD}

Dr. Justiz is a Consultant for St. Jude and Medtronic.

\section{Alan D. Kaye, MD, PhD}

Dr. Kaye is a speaker for Depomed and Merck, Inc.

\section{Nalini Sehgal, MD}

Dr Sehgal is a consultant for Pfizer Inc, Mallinckrodt Inc and Pacira Inc.

\section{Sanford M. Silverman, MD}

Dr. Silverman is a speaker for Daitchi Sankyo, Purdue, Depomed, and Endo.

\section{Thomas T. Simopoulos, MD}

Dr. Simopoulos is a consultant for Nevro Corp, St. Jude Medical, and Boston Scientific.

\section{Konstantin Slavin, MD}

Dr. Slavin is a consultant for Medtronic, St. Jude Medical, Boston Scientific, Nevro, SPR Therapeutics, Nuvectra, and Stimrelieve.

\section{Peter S. Staats, MD}

Dr. Staats receives grants from Boston Scientific, Halyard, and St. Jude, an honorarium from Nevro, Halyard, Medtronic, and St. Jude, patents from NeurogesX, royalties from multiple books and NeurogesX, and has investment in Electrocore.

\section{Ricardo Vallejo, MD}

Dr. Vallejo is a consultant and advisory board member of Halyard; and has received a basic science grant from Nevro. 
Appendix 1. Sample controlled substance agreement.

We are committed to doing all we can to treat your chronic pain condition. In some cases, controlled substances are used as a therapeutic option in the management of chronic pain and related anxiety and depression, these substances are strictly regulated by both state and federal agencies. This agreement is a tool to protect you and your physician by establishing guidelines, within the laws, for proper controlled substance use. The words "we" and "our" refer to the facility, and the words "I", "you", "your", "me", or "my" refer to you, the patient.

1. i. I understand that chronic opioid therapy has been associated with not only addiction and abuse, but also multiple medical problems including the suppression of endocrine function resulting in low hormonal levels in men and women which may affect mood, stamina, sexual desire, and physical and sexual performance.

ii. Opioids and other controlled substances during pregnancy are associated with multiple effects on the baby including birth defects and physical dependency for the baby on opioids upon delivery. I will immediately contact my obstetrician and this office to inform them of my pregnancy.

iii. I have been informed that long-term and/or high doses of pain medications may also cause increased levels of pain known as opioid-induced hyperalgesia (pain medicine causing more pain). Simple touch will be felt as pain and pain gradually increases in intensity and the pain's location is all over the body. I understand that opioid-induced hyperalgesia is a normal, expected result of using these medicines for a long period of time. This is only treated with the addition of nonsteroidal anti-inflammatory drugs such as Advil, Aleve, etc., or by reducing or stopping opioids.

iv. I understand that physical dependence is not the same as addiction. I am aware physical dependence means that if my pain medicine use is markedly decreased, stopped, or reversed by some of the agents mentioned above, I will experience a withdrawal syndrome. This means I may have any or all of the following: runny nose, yawning, large pupils, goose bumps, abdominal pain and cramping, diarrhea, irritability, aches throughout my body, and a flu-like feeling. I am aware that opioid withdrawal is uncomfortable, can cause severe anxiety, rapid heart rate, and profound blood pressure changes, and could even result in heart attack, stroke, or death.

v. I am aware that tolerance to analgesia means that I may require more medicine to get the same amount of pain relief. I am aware that tolerance to analgesia does not seem to be a big problem for most patients with chronic pain; however, it has been seen and may happen to me. I have been informed that tolerance and physical dependence go hand in hand. Tolerance or failure to respond well to opioids may cause my doctor to choose another form of treatment, reduce the dose, or stop them.

2. i. All controlled substances must come from one of the physicians from this practice, by the covering physician, unless specific authorization is obtained for an exception.

ii. I understand that I must inform this office, of all drugs that I am taking, have purchased, or have obtained, even over-the-counter medications. Failure to do so may result in drug interactions or overdoses that could result in harm to me, including death.

iii. I will not seek prescriptions for controlled substances from any other physician, health care provider, or dentist, except for unrelated emergencies, trauma, and surgery. I understand it is unlawful to be prescribed the same controlled medication by more than one physician at a time without each physician's knowledge. Opioid-based cough suppressants, sleeping pills including sedatives, when combined with other prescribed medications utilized in pain management could result in toxicity including death. 
iv. I also understand that it is unlawful to obtain or to attempt to obtain a prescription for a controlled substance by knowingly misrepresenting facts to a physician or his/her staff or knowingly withholding facts from a physician or his/her staff (including failure to inform the physician or his/her staff of all controlled substances that I have been prescribed).

3. All controlled substances must be obtained at the same pharmacy if possible. Should the need arise to change pharmacies, our office must be informed. The pharmacy that I have selected is:

4. i. I will not share, sell, or otherwise permit others, including my spouse or family members, to have access to any controlled substances that I have been prescribed.

ii. Early refills will not be given. I will not consume excessive amounts, I will follow prescribed instructions, and remain compliant to all aspects of treatment. Renewals are based upon keeping scheduled appointments. Please do not call for refills after hours or on weekends.

iii. Medication changes will not be made between appointments except in emergency situations, which will be determined by the physician.

5. Unannounced pill counts, random urine or serum tests, or planned drug screening may be requested from you and your cooperation is required. The presence of unauthorized substances in urine or serum toxicology screens may result in your discharge from treatment by the facility and its physicians and staff.

6. It is advised that after beginning opioid treatment, or after a dose increase a patient should not drive for at least 4-5 days, possibly longer based on individual response. it is also being advised that with prescribed chronic opioids, it is being warned not to drive or engage in potentially dangerous work or other activities until a patient becomes tolerant to any sedative properties of the medications prescribed and have had enough time to understand the medications ability to impair or not impair my driving abilities.

7. I will not consume excessive amounts of alcohol in conjunction with controlled substances. I will not use, purchase, or otherwise obtain any other legal drugs except as specifically authorized by the physician, as set forth in Section 1 above. I will not use, purchase, or otherwise obtain any illegal drugs, including marijuana, cocaine, etc. I understand that driving while under the influence of any substance, including a prescribed controlled substance or any combination of substances (e.g., alcohol and prescription drugs), which impairs my driving ability may result in DUI charges.

8. Medications or written prescriptions may not be replaced if they are lost, stolen, get wet, are destroyed, left on an airplane, etc. If your medication has been stolen, it will not be replaced unless explicit proof is provided with direct evidence from authorities. A report narrating what you told the authorities is not enough.

9. Respiratory depression can occur and can be fatal if not treated immediately by calling 911 or going to an emergency room. A patient will be provided based on medical necessity or upon request with an opioid antagonist prescription EVZIO 0.4MG/0.4ML AUTO INJECTOR 2PK or Narcan Nasal Spray. (Opioid Overdose Antidote Naloxone) to inject if experiences signs or symptoms of overdose.

10. I understand that my provider will be verifying that I am receiving controlled substances from only one prescriber and only one pharmacy by checking the Prescription Monitoring Drug Programs web site periodically throughout my treatment period.

11. In the event I am arrested or incarcerated in relation to legal or illegal drugs (including alcohol), or overdosed on controlled substances, controlled substances will be withheld for an appropriate period. 
12. I understand that failure to adhere to these policies may result in cessation of therapy with controlled substances prescribed by this physician and other physicians at the facility and that law enforcement officials may be contacted.

13. I also understand that the prescribing physician has permission to discuss all diagnostic and treatment details, including medications, with dispensing pharmacists, other professionals who provide your health care, or appropriate drug and law enforcement agencies for the purpose of maintaining accountability.

14. I affirm that I have full right and power to sign and to be bound by this agreement, that I have read it, and understand and accept all of its terms. A copy of this document has been given to me.

Patient's full name

Patient's signature

Physician's signature
Date

Date 


\section{References}

1. Atluri S, Sudarshan G, Manchikanti L. Assessment of the trends in medical use and misuse of opioid analgesics from 2004 to 2011. Pain Physician 2014; 17:E119-E128.

2. Dart RC, Surratt HL, Cicero TJ, Parrino MW, Severtson SG, Bucher-Bartelson B, Green JL. Trends in opioid analgesic abuse and mortality in the United States. N Engl J Med 2015; 372:241-248.

3. Deyo RA, Von Korff M, Duhrkoop D. Opioids for low back pain. BMJ 2015; 350:g6380.

4. Kolodny A, Courtwright DT, Hwang CS, Kreiner P, Eadie JL, Clark TW, Alexander GC. The prescription opioid and heroin crisis: A public health approach to an epidemic of addiction. Annu Rev Public Health 2015; 36:559-574.

5. Betses M, Brennan T. Abusive prescribing of controlled substances - a pharmacy view. N Engl J Med 2013; 369:989-991.

6. Jones CM, Lurie P, Woodcock J. Addressing prescription opioid overdose: Data support a comprehensive policy approach. JAMA 2014; 312:1733-1734.

7. Nelson LS, Juurlink DN, Perrone J. Addressing the opioid epidemic. JAMA 2015; 314:1453-1454.

8. Han B, Compton WM, Jones CM, Cai R. Nonmedical prescription opioid use and use disorders among adults aged 18 through 64 years in the United States, 2003-2013. JAMA 2015; 314:1468-1478.

9. Clarke JL, Skoufalos A, Scranton R. The American opioid epidemic: Population health implications and potential solutions. Report from the National Stakeholder Panel. Popul Health Manag 2016; 19:S1-Sio.

10. Buckley N. The prescription opioid epidemic: A call to action for our profession. Can J Anaesth 2016; 63:8-11.

11. Manchikanti L, Abdi S, Atluri S, Balog CC, Benyamin RM, Boswell MV, Brown KR, Bruel BM, Bryce DA, Burks PA, Burton AW, Calodney AK, Caraway DL, Cash KA, Christo PJ, Damron KS, Datta S, Deer TR, Diwan S, Eriator I, Falco FJE, Fellows F, Geffert S, Gharibo CG, Glaser SE, Grider JS, Hameed H, Hameed M, Hansen $H$, Harned ME, Hayek SM, Helm II S, Hirsch JA, Janata JW, Kaye AD, Kaye AM, Kloth DS, Koyyalagunta D, Lee M, Malla Y, Manchikanti KN, McManus CD, Pampati V, Parr AT, Pasupuleti R, Patel VB, Sehgal N, Silverman SM, Singh V, Smith HS, Snook LT, Solanki DR, Tra- cy DH, Vallejo R, Wargo BW. American Society of Interventional Pain Physicians (ASIPP) guidelines for responsible opioid prescribing in chronic non-cancer pain: Part I - Evidence assessment. Pain Physician 2012; 15:S1-S66.

12. Manchikanti L, Abdi S, Atluri S, Balog CC, Benyamin RM, Boswell MV, Brown KR, Bruel BM, Bryce DA, Burks PA, Burton AW, Calodney AK, Caraway DL, Cash KA, Christo PJ, Damron KS, Datta S, Deer TR, Diwan S, Eriator I, Falco FJE, Fellows F, Geffert S, Gharibo CG, Glaser SE, Grider JS, Hameed H, Hameed M, Hansen $\mathrm{H}$, Harned ME, Hayek SM, Helm II S, Hirsch JA, Janata JW, Kaye AD, Kaye AM, Kloth DS, Koyyalagunta D, Lee M, Malla Y, Manchikanti KN, McManus CD, Pampati V, Parr AT, Pasupuleti R, Patel VB, Sehgal N, Silverman SM, Singh V, Smith HS, Snook LT, Solanki DR, Tracy $\mathrm{DH}$, Vallejo R, Wargo BW. American Society of Interventional Pain Physicians (ASIPP) guidelines for responsible opioid prescribing in chronic non-cancer pain: Part 2 - Guidance. Pain Physician 2012; 15:S67-S116.

13. Gwira Baumblatt JA, Wiedeman C, Dunn JR, Schaffner W, Paulozzi LJ, Jones TF. High-risk use by patients prescribed opioids for pain and its role in overdose deaths. JAMA Intern Med 2014; 174:796-801.

14. Bateman BT, Choudhry NK. Limiting the duration of opioid prescriptions: Balancing excessive prescribing and the effective treatment of pain. JAMA Intern Med 2016; 176:583-584.

15. Wright AP, Becker WC, Schiff GD. Strategies for flipping the script on opioid overprescribing. JAMA Intern Med 2016; 176:7-8.

16. Manchikanti L, Kaye AM, Kaye AD. Current state of opioid therapy and abuse. Curr Pain Headache Rep 2016; 20:34.

17. Ray WA, Chung CP, Murray KT, Hall K, Stein CM. Prescription of long-acting opioids and mortality in patients with chronic noncancer pain. JAMA 2016; 315:2415-2423.

18. Cheung CW, Qiu Q, Choi SW, Moore B, Goucke R, Irwin M. Chronic opioid therapy for chronic non-cancer pain: A review and comparison of treatment guidelines. Pain Physician 2014; 17:401-414.

19. Duthey B, Scholten W. Adequacy of opioid analgesic consumption at country, global, and regional levels in 2010, its relationship with development level, and changes compared with 2006. J Pain Symptom Manage 2014; 47:283-297.

20. Weisberg DF, Becker WC, Fiellin DA, Stannard C. Prescription opioid misuse in the United States and the United Kingdom: Cautionary lessons. Int ] Drug Policy 2014; 25:1124-1130.

21. Murphy Y, Goldner EM, Fischer B. Prescription opioid use, harms and interventions in Canada: A review update of new developments and findings since 2010. Pain Physician 2015; 18:E605-E614.

22. Fischer $\mathrm{B}$, Jones $\mathrm{W}$, Murphy $\mathrm{Y}$, lalomiteanu A, Rehm J. Recent developments in prescription opioid-related dispensing and harm indicators in Ontario, Canada. Pain Physician 2015; 18:E659-E662.

23. Birke H, Kurita GP, Sjøgren P, Højsted J, Simonsen MK, Juel K, Ekholm O. Chronic non-cancer pain and the epidemic prescription of opioids in the Danish population: Trends from 2000 to 2013. Acta Anaesthesiol Scand 2016; 6o:623-633.

24. McHugh RK, Nielsen S, Weiss RD. Prescription drug abuse: From epidemiology to public policy. J Subst Abuse Treat 2015; 48:1-7.

25. Gomes T, Mamdani MM, Paterson JM, Dhalla IA, Juurlink DN. Trends in highdose opioid prescribing in Canada. Can Fam Physician 2014; 60:826-832.

26. Currow DC, Phillips J, Clark K. Using opioids in general practice for chronic non-cancer pain: An overview of current evidence. Med J Aust 2016; 204:305-309.

27. Timmerman L, Stronks DL, Groeneweg JG, Huygen FJ. Prevalence and determinants of medication non-adherence in chronic pain patients: A systematic review. Acta Anaesthesiol Scand 2016; 60:416-431.

28. Marteau D, McDonald R, Patel K. The relative risk of fatal poisoning by methadone or buprenorphine within the wider population of England and Wales. BM] Open 2015; 5:e0o7629.

29. Islam MM, McRae IS, Mazumdar S, Taplin S, McKetin R. Prescription opioid analgesics for pain management in Australia: Twenty years of dispensing. Intern Med J 2016; 46:955-963.

30. United Nations Office on Drugs and Crime. Ensuring availability of controlled medications for the relief of pain and preventing diversion and abuse: Striking the right balance to achieve the optimal public health outcome. Discussion paper based on a scientific work- 
shop. UNODC, Vienna, Austria, January 18-19, 2011.

www.unodc.org/docs/treatment/Pain/ Ensuring_availability_of_controlled_ medications_FINAL_15_March_CND_ version.pdf

31. Häuser W, Petzke F, Radbruch L, Tölle TR. The opioid epidemic and the longterm opioid therapy for chronic noncancer pain revisited: A transatlantic perspective. Pain Manag 2016; 6:249-263.

32. Scholten W, Henningfield JE. Negative outcomes of unbalanced opioid policy supported by clinicians, politicians, and the media. J Pain Palliat Care Pharmacother 2016; 30:4-12.

33. Pergolizzi JV, Raffa RB, LeQuang JA. The Centers for Disease Control and Prevention opioid guidelines: Potential for unintended consequences and will they be abused? J Clin Pharm Ther 2016; 41:592-593.

34. Dijulio B, Wu B, Brode M; Kaiser Family Foundation. The Washington Post/Kaiser Family Foundation survey of longterm prescription painkiller users and their household members. December 2016.

http://files.kff.org/attachment/Surveyof-Long-Term-Prescription-PainkillerUsers-and-Their-Household-Members

35. Pizzi DM. CDC opioid guideline: Put away the prescription pain. Pain Medicine News, March 16, 2016.

www.painmedicinenews.com/WebOnly/Article/03-16/CDC-OpioidGuideline-Puts-Pressure-on-PCPs-ToPut-Away-the-Prescription-Pad/35657/ ses $=$ ogst

36. Rudd RA, Aleshire N, Zibbell JE, Gladden RM. Increases in drug and opioid overdose deaths - United States, 2000 - 2014. MMWR Morb Mortal Wkly Rep 2016; 64:1378-1382.

37. Rudd RA, Seth P, David F, Scholl L. Increases in drug and opioid-involved overdose deaths - United States, 20102015. MMWR Morb Mortal Wkly Rep 2016; 65:1445-1452.

38. Chen JH, Humphreys K, Shah $\mathrm{NH}$, Lembke A. Distribution of opioids by different types of medicare prescribers. JAMA Intern Med 2016; 176:259-261.

39. Salsitz EA. Chronic pain, chronic opioid addiction: A complex nexus. J Med Toxicol 2016; 12:54-57.

40. Rastegar DA, Walley AY. Preventing prescription opioid overdose deaths. J Gen Intern Med 2013; 28:1258-1259.

41. Manchikanti L, Atluri S, Hansen H, Be- nyamin RM, Falco FJE, Helm II S, Kaye $A D$, Hirsch JA. Opioids in chronic noncancer pain: Have we reached a boiling point yet? Pain Physician 2014; 17:E1-E10.

42. Dowell D, Haegerich TM, Chou R. CDC Guideline for prescribing opioids for chronic pain - United States, 2016. JAMA 2016; 315:1624-1645.

43. International Narcotics Control Board. Annual Reports, tables of reported statistics. Part Four: Statistical information on narcotic drugs. 2011.

www.incb.org/documents/NarcoticDrugs/Technical-Publications/2011/ Part_FOUR_Complete_English-NARReport-2011.pdf

44. International Narcotics Control Board Annual Reports. 2010 to 2014

www.incb.org/documents/NarcoticDrugs/Technical-Publications/2015/ part_4_tables_EFS.pdf

45. Goodnough A, Tavernise S. Opioid prescriptions drop for the first time in two decades. New York Times, May 20, 2016. www.nytimes.com/2016/05/21/health/ opioid-prescriptions-drop-for-firsttime-in-two-decades.html?_r=o

46. IMS Health. Top-line market data: Global prescription sales information.

www.imshealth.com/en/about-us/news/ top-line-market-data

47. Symphony Health Solutions. http:// symphonyhealth.com/solutions/\#

48. Manchikanti L, Whitfield E, Pallone F. Evolution of the National All Schedules Prescription Electronic Reporting Act (NASPER): A public law for balancing treatment of pain and drug abuse and diversion. Pain Physician 2005; 8:335-347.

49. Department of Veterans Affairs (VA)/Department of Defense (DOD). VA/DoD clinical practice guideline for management of opioid therapy for chronic pain. Washington, DC: Veterans Administration; 2010.

www.va.gov/painmanagement/docs/ cpg_opioidtherapy_fulltext.pdf

50. Interagency Guideline on Prescribing Opioids for Pain. Washington State Agency Medical Directors' Group (AMDG) in collaboration with an Expert Advisory Panel, Actively Practicing Providers, Public Stakeholders, and Senior State Officials, 3rd Edition, June 2015. www.agencymeddirectors.wa.gov/ Files/2015AMDGOpioidGuideline.pdf

51. Kahan M, Wilson L, Mailis-Gagnon A, Srivastava A; National Opioid Use Guideline Group. Canadian guideline for safe and effective use of opioids for chronic noncancer pain: Clinical summary for family physicians. Part 2: Special populations. Can Fam Physician 2011; 57:1269-1276, e419-e428.

52. Kertesz SG. Turning the tide or riptide? The changing opioid epidemic. Subst Abus 2016 Nov 18:0. [Epub ahead of print]

53. U.S. Department of Health and Human Services. News Release. HSS takes strong steps to address opioid-drug related overdose, death and dependence. March 26, 2015.

www.hhs.gov/about/news/2015/03/26/ hhs-takes-strong-steps-to-address-opioid-drug-related-overdose-death-anddependence.html\#

54. Centre for Clinical Practice at NICE. NICE Clinical Guideline 173. Neuropathic pain in adults: Pharmacological management in non-specialist settings. Issued: November 2013 Updated: December 2014.

www.nice.org.uk/guidance/cg173/ evidence/neuropathic-pain-pharmacological-management-full-guideline-191621341

55. Chou R, Fanciullo GJ, Fine PG, Adler JA, Ballantyne JC, Davies P, Donovan MI, Fishbain DA, Foley KM, Fudin J, Gilson AM, Kelter A, Mauskop A, O'Connor PG, Passik SD, Pasternak GW, Portenoy RK, Rich BA, Roberts RG, Todd KH, Miaskowski C; American Pain Society-American Academy of Pain Medicine Opioids Guidelines Panel. Clinical guidelines for the use of chronic opioid therapy in chronic noncancer pain. J Pain 2009; 10:113-130.

56. Graham R, Mancher M, Wolman DM, Greenfield S, Steinberg E (eds); Committee on Standards for Developing Trustworthy Clinical Practice Guidelines; Institute of Medicine. Clinical Practice Guidelines We Can Trust. The National Academies Press, Washington, DC, 2011.

57. National Guideline Clearinghouse Extent Adherence to Trustworthy Standards (NEATS) instrument.

58. Manchikanti L, Falco FJE, Benyamin R M, Kaye AD, Boswell MV, Hirsch JA. A modified approach to grading of evidence. Pain Physician 2014; 17:E319-E325.

59. Harris RP, Helfand M, Woolf SH, Lohr KN, Mulrow CD, Teutsch SM, Atkins D; Methods Work Group, Third US Preventive Services Task Force. Current methods of the US Preventive Services Task Force. Am J Prevent Med 2001; 20:21-35.

6o. Institute of Medicine (IOM). Relieving 
Pain in America: A Blueprint for Transforming Prevention, Care, Education, and Research. The National Academies Press, Washington, DC, June 29, 2011.

www.iom.edu/ /media/Files/Report\%20Files/2011/Relieving-Pain-inAmerica-A-Blueprint-for-TransformingPrevention-Care-Education-Research/ Pain\%20Research\%202011\%20Report\%2oBrief.pdf

61. Gaskin DJ, Richard P. The economic costs of pain in the United States. J Pain 2012; 13:715-724.

62. Manchikanti L, Atluri S, Candido KD, Boswell MV, Simopoulos TT, Grider JS, Falco FJE, Hirsch JA. Zohydro' ${ }^{\mathrm{TM}}$ approval by Food and Drug Administration: Controversial or frightening? Pain Physician 2014; 17:E437-E450.

63. Martin BI, Turner JA, Mirza SK, Lee MJ, Comstock BA, Deyo RA. Trends in health care expenditures, utilization, and health status among US adults with spine problems, 1997 -2006. Spine (Phila $\mathrm{Pa}$ 1976) 2009; 34:2077-2084.

64. Freburger JK, Holmes GM, Agans RP, Jackman AM, Darter JD, Wallace AS, Castel LD, Kalsbeek WD, Carey TS. The rising prevalence of chronic low back pain. Arch Intern Med 2009; 169:251-258.

65. Dieleman JL, Baral R, Birger $M$, Bui AL, Bulchis A, Chapin A, Hamavid H, Horst C, Johnson EK, Joseph J, Lavado R, Lomsadze L, Reynolds A, Squires E, Campbell $M$, DeCenso $B$, Dicker $D$, Flaxman AD, Gabert R, Highfill T, Naghavi M, Nightingale N, Templin T, Tobias MI, Vos T, Murray CJ. US spending on personal health care and public health, 1996-2013. JAMA 2016; 316:2627-2646.

66. Hoy DG, Bain C, Williams G, March L, Brooks P, Blyth F, Woolf A, Vos T, Buchbinder R. A systematic review of the global prevalence of low back pain. Arthritis Rheum 2012; 64:2028-2037.

67. Hoy DG, Protani M, De R, Buchbinder R. The epidemiology of neck pain. Best Pract Res Clin Rheumatol 2010; 24:783-792.

68. Bekkering GE, Bala MM, Reid K, Kellen E, Harker J, Riemsma R, Huygen FJ, Kleijnen J. Epidemiology of chronic pain and its treatment in The Netherlands. Neth J Med 2011; 69:141-153.

69. Reid KJ, Harker J, Bala MM, Truyers C, Kellen E, Bekkering GE, Kleijnen J. Epidemiology of chronic non-cancer pain in Europe: Narrative review of prevalence, pain treatments and pain impact. Curr Med Res Opin 2011; 27:449-462.
70. Sjøgren P, Ekholm O, Peuckmann V, Grønbæk M. Epidemiology of chronic pain in Denmark: An update. Eur J Pain 2009; 13:287-292.

71. Verhaak PF, Kerssens JJ, Dekker J, Sorb MJ, Bensing JM. Prevalence of chronic benign pain disorder among adults: A review of the literature. Pain 1998; 77:231-239.

72. Manchikanti L, Singh V, Falco FJE, Benyamin RM, Hirsch JA. Epidemiology of low back pain in adults. Neuromodulation 2014; 17:3-10.

73. Breivik H, Collett B, Ventafridda V, Cohen R, Gallacher D. Survey of chronic pain in Europe: Prevalence, impact on daily life, and treatment. Eur J Pain 2006; 10:287-333.

74. Blyth FM, Rochat S, Cumming RG, Creasey H, Handelsman DJ, Le Couteur DG, Naganathan V, Sambrook PN, Seibel MJ, Waite LM. Pain, frailty and comorbidity on older men: The CHAMP study. Pain 2008; 140:224-230.

75. Cassidy JD, Carroll LJ, Côté P. The Saskatchewan Health and Back Pain Survey. The prevalence of low back pain and related disability in Saskatchewan adults. Spine (Phila Pa 1976) 1998; 23:1860-1867.

76. Côté $P$, Cassidy JD, Carroll L. The Saskatchewan Health and Back Pain Survey. The prevalence of neck pain and related disability in Saskatchewan adults. Spine (Phila Pa 1976) 1998; 23:1689-1698.

77. Leboeuf-Yde C, Nielsen J, Kyvik KO, Fejer R, Hartvigsen J. Pain in the lumbar, thoracic or cervical regions: Do age or gender matter? A population-based study of 34,902 Danish twins $20-71$ years of age. BMC Musculoskelet Disord 2009; 10:39.

78. Côté P, Cassidy JD, Carroll L. The factors associated with neck pain and its related disability in the Saskatchewan population. Spine (Phila Pa 1976) 2000; 25:1109-1117.

79. Merskey H, Bogduk N. Task Force on Taxonomy of the International Association for the Study of Pain. Classification of Chronic Pain: Descriptions of Chronic Pain Syndromes and Definition of Pain Terms. 2nd ed. IASP Press, Seattle, WA, 1994.

8o. Manchikanti L, Falco FJE, Singh V, Benyamin RM, Racz GB, Helm II S, Caraway DL, Calodney AK, Snook LT, Smith HS, Gupta S, Ward SP, Grider JS, Hirsch JA. An update of comprehensive evidence-based guidelines for interventional techniques of chronic spinal pain. Part I: Introduction and general consid- erations. Pain Physician 2013; 16:S1-S48.

81. Manchikanti L, Abdi S, Atluri S, Benyamin RM, Boswell MV, Buenaventura RM, Bryce DA, Burks PA, Caraway DL, Calodney AK, Cash KA, Christo PJ, Cohen SP, Colson J, Conn A, Cordner HJ, Coubarous S, Datta S, Deer TR, Diwan SA, Falco FJE, Fellows B, Geffert SC, Grider JS, Gupta S, Hameed H, Hameed M, Hansen H, Helm II S, Janata JW, Justiz R, Kaye AD, Lee M, Manchikanti KN, McManus CD, Onyewu O, Parr AT, Patel VB, Racz GB, Sehgal N, Sharma M, Simopoulos TT, Singh V, Smith HS, Snook LT, Swicegood J, Vallejo R, Ward SP, Wargo BW, Zhu J, Hirsch JA. An update of comprehensive evidence-based guidelines for interventional techniques of chronic spinal pain: Part II: Guidance and recommendations. Pain Physician 2013; 16:S49-S283.

82. Elliott AM, Smith BH, Penny KI, Smith WC, Chambers WA. The epidemiology of chronic pain in the community. Lancet 1999; 354:1248-1252.

83. Gureje O, Von Korff M, Simon GE, Gater R. Persistent pain and well-being: A World Health Organization study in primary care. JAMA 1998; 280:147-151.

84. Moulin DE, Clark AJ, Speechley M, Morley-Forster PK. Chronic pain in Canada - prevalence, treatment, impact and the role of opioid analgesia. Pain Res Manag 2002; 7:179-184.

85. U.S. Burden of Disease Collaborators. The state of US health, $1990-2010$ : Burden of diseases, injuries, and risk factors. JAMA 2013; 310:591-608.

86. Manchikanti L, Singh V, Datta S, Cohen SP, Hirsch JA. Comprehensive review of epidemiology, scope, and impact of spinal pain. Pain Physician 2009; 12:E35-E70.

87. Hoy D, March L, Brooks P, Blyth F, Woolf A, Bain C, Williams G, Smith E, Vos T, Barendregt J, Murray C, Burstein $\mathrm{R}$, Buchbinder R. The global burden of low back pain: Estimates from the Global Burden of Disease 2010 study. Ann Rheum Dis 2014; 73:968-974.

88. Hoy D, March L, Woolf A, Blyth F, Brooks $\mathrm{P}$, Smith E, Vos T, Barendregt J, Blore J, Murray C, Burstein R, Buchbinder R. The global burden of neck pain: Estimates from the global burden of disease 2010 study. Ann Rheum Dis 2014; 73:1309-1315.

89. Dhalla IA, Mamdani MM, Sivilotti ML, Kopp A, Qureshi O, Juurlink DN. Prescribing of opioid analgesics and related mortality before and after the introduc- 
tion of long-acting oxycodone. CMA] 2009; 181:891-896.

90. Deyo RA, Smith DH, Johnson ES, Donovan M, Tillotson CJ, Yang X, Petrik AF, Dobscha SK. Opioids for back pain patients: Primary care prescribing patterns and use of services. J Am Board Fam Med 2011; 24:717-727.

91. Manchikanti L, Fellows B, Ailinani H, Pampati V. Therapeutic use, abuse, and nonmedical use of opioids: A tenyear perspective. Pain Physician 2010; 13:401-435.

92. Dhalla IA, Persaud N, Juurlink DN. Facing up to the prescription opioid crisis. BM] 2011; 343:d5142.

93. Volkow ND, McLellan TA, Cotto $\mathrm{JH}$. Characteristics of opioid prescriptions in 2009. JAMA 2011; 305:1299-1301.

94. Martell BA, O'Connor PG, Kerns RD, Becker WC, Morales KH, Kosten TR, Fiellin DA. Systematic review: Opioid treatment for chronic back pain: Prevalence, efficacy, and association with addiction. Ann Intern Med 2007; 146:116-127.

95. Caudill-Slosberg MA, Schwartz LM, Woloshin S. Office visits and analgesic prescriptions for musculoskeletal pain in US: 1980 vs. 2000. Pain 2004; 109:514-519.

96. Franklin GM, Mai J, Wickizer T, Turner JA, Fulton-Kehoe D, Grant L. Opioid dosing trends and mortality in Washington State workers' compensation, 1996 2002. Am J Ind Med 2005; 48:91-99.

97. Luo X, Pietrobon R, Hey L. Patterns and trends in opioid use among individuals with back pain in the United States. Spine (Phila Pa 1976) 2004; 29:884-891.

98. Xu J, Kochanek KD, Murphy SL, TejadaVera B. Deaths: Final data for 2007. National vital statistics reports; Vol. $58 \mathrm{No}$. 19. National Center for Health Statistics, Hyattsville, MD, 2010.

www.cdc.gov/nchs/data/nvsr/nvsr58/ nvsr58_o1.pdf

99. Zerzan JT, Morden NE, Soumerai $S$, Ross-Degnan D, Roughead E, Zhang F, Simoni-Wastila L, Sullivan SD. Trends and geographic variation of opiate medication use in state Medicaid feefor-service programs, 1996 to 2002. Med Care 2006; 44:1005-1010.

100. Edlund MJ, Martin BC, Devries A, Fan M-Y, Braden JB, Sullivan MD. Trends in use of opioids for chronic noncancer pain among individuals with mental health and substance use disorders: The TROUP Study. Clin J Pain 2010; 26:1-8.

101. Sullivan MD, Edlund MJ, Fan MY,
Devries A, Brennan Braden J, Martin BC. Risks for possible and probable opioid misuse among recipients of chronic opioid therapy in commercial and medicaid insurance plans: The TROUP Study. Pain 2010; 150:332-339.

102. Fleming MF, Balousek SL, Klessig CL, Mundt MP, Brown DD. Substance use disorders in a primary care sample receiving daily opioid therapy. J Pain 2007; 8:573-582.

103. National Center for Health Statistics. Health, United States, 2008 with chart book. National Center for Health Statistics, Hyattsville, MD, 2009.

104. Centers for Disease Control and Prevention (CDC). Adult use of prescription opioid pain medications - Utah, 2008. MMWR Morb Mortal Wkly Rep 2010; 59:153-157.

105. Sullivan MD, Edlund MJ, Fan MY, Devries A, Brennan Braden J, Martin BC. Trends in use of opioids for noncancer pain conditions $2000-2005$ in commercial and Medicaid insurance plans: The TROUP study. Pain 2008; 138:440-449.

106. Vogt MT, Kwoh CK, Cope DK, Osial TA, Culyba M, Starz TW. Analgesic usage for low back pain: Impact on health care costs and service use. Spine (Phila Pa 1976) 2005; 30:1075-1081.

107. Cullen KA, Hall MJ, Golosinsky A. Ambulatory surgery in the United States, 2006. Natl Health Stat Report 2009; 11:1-25.

108. Alam A, Gomes T, Zheng H, Mamdami MM, Juurlink DN, Bell CM. Long-term analgesic use after low-risk surgery: A retrospective cohort study. Arch Intern Med 2012; 172:425-430.

109. Paulozzi LJ, Strickler GK, Kreiner PW Koris CM; Centers for Disease Control and Prevention (CDC). Controlled substance prescribing patterns - Prescription Behavior Surveillance System, eight states, 2013. MMWR Surveill Summ 2015; 64:1-14.

110. Manchikanti L, Cash KA, Malla Y, Pampati $\mathrm{V}$, Fellows B. A prospective evaluation of psychotherapeutic and illicit drug use in patients presenting with chronic pain at the time of initial evaluation. Pain Physician 2013; 16:E1-E13.

111. Calcaterra SL, Yamashita TE, Min SJ, Keniston A, Frank JW, Binswanger IA Opioid prescribing at hospital discharge contributes to chronic opioid use. J Gen Intern Med 2016; 31:478-485.

112. Jena AB, Goldman D, Weaver L, KaracaMandic P. Opioid prescribing by multi- ple providers in Medicare: Retrospective observational study of insurance claims. BM] 2014; 348:g1393.

113. Campbell Cl, Weisner C, Leresche L, Ray GT, Saunders K, Sullivan MD, Banta-Green CJ, Merrill JO, Silverberg MJ, Boudreau D, Satre DD, Von Korff M. Age and gender trends in long-term opioid analgesic use for noncancer pain. Am J Public Health 2010; 100:2541-2547.

114. Morden NE, Munson JC, Colla CH, Skinner JS, Bynum JP, Zhou W, Meara E. Prescription opioid use among disabled Medicare beneficiaries: Intensity, trends, and regional variation. Med Care 2014; 52:852-859.

115. Garg RK, Fulton-Kehoe D, Turner JA, Bauer AM, Wickizer T, Sullivan MD, Franklin GM. Changes in opioid prescribing for Washington workers' compensation claimants after implementation of an opioid dosing guideline for chronic noncancer pain: 2004 to 2010. J Pain 2013; 14:1620-1628.

116. Centers for Disease Control and Prevention (CDC). Vital signs: Overdoses of prescription opioid pain relievers, United States, 1999 - 2008. MMWR Morb Mortal Wkly Rep 2011; 60:1487-1492.

117. Sabel J. Unintentional prescription opioid overdose deaths Washington 1995 2010. Washington State Department of Health.

118. Franklin GM, Mai J, Turner J, Sullivan M, Wickizer T, Fulton-Kehoe D. Bending the prescription opioid dosing and mortality curves: Impact of the Washington State opioid dosing guideline. Am J Ind Med 2012; 55:325-331.

119. Eriksen J, Jensen MK, Sjøgren P, Ekholm $\mathrm{O}$, Rasmussen NK. Epidemiology of chronic non-malignant pain in Denmark. Pain 2003; 106:221-228.

120. Kantor ED, Rehm CD, Haas JS, Chan AT, Giovannucci EL. Trends in prescription drug use among adults in the United States from 1999 - 2012. JAMA 2015; 314:1818-1831.

121. West NA, Severtson SG, Green JL, Dart RC. Trends in abuse and misuse of prescription opioids among older adults. Drug Alcohol Depend 2015; 149:117-121.

122. West NA, Dart RC. Prescription opioid exposures and adverse outcomes among older adults. Pharmacoepidemiol Drug Saf 2016; 25:539-544.

123. Rauenzahn S, Del Fabbro E. Opioid management of pain: The impact of the prescription opioid abuse epidemic. Curr Opin Support Palliat Care 2014; 8:273-278. 
124. Cheatle MD. Prescription opioid misuse, abuse, morbidity, and mortality: Balancing effective pain management and safety. Pain Med 2015; 16:S3-S8.

125. Tournebize J, Gibaja V, Muszczak A, Kahn JP. Are physicians safely prescribing opioids for chronic noncancer pain? A systematic review of current evidence. Pain Pract 2016; 16:370-383.

126. Kanouse $A B$, Compton P. The epidemic of prescription opioid abuse, the subsequent rising prevalence of heroin use, and the federal response. ] Pain Palliat Care Pharmacother 2015; 29:102-114.

127. Compton WM, Boyle M, Wargo E. Prescription opioid abuse: Problems and responses. Prev Med 2015; 80:5-9.

128. Wilkerson RG, Kim HK, Windsor TA, Mareiniss DP. The opioid epidemic in the United States. Emerg Med Clin North Am 2016; 34:e1-e23.

129. Portenoy RK, Foley KM. Chronic use of opioid analgesics in non-malignant pain: Report of 38 cases. Pain 1986; 25:171-186.

130. Sullivan MD, Bauer AM, Fulton-Kehoe D, Garg RK, Turner JA, Wickizer T, Franklin GM. Trends in opioid dosing among Washington State Medicaid patients before and after opioid dosing guideline implementation. J Pain 2016; 17:561-568.

131. Fulton-Kehoe D, Sullivan MD, Turner JA, Garg RK, Bauer AM, Wickizer TM, Franklin GM. Opioid poisonings in Washington State Medicaid: Trends, dosing, and guidelines. Med Care 2015; 53:679-685.

132. Garcia MM, Angelini MC, Thomas T, Lenz K, Jeffrey P. Implementation of an opioid management initiative by a state Medicaid program. J Manag Care Spec Pharm 2014; 20:447-454.

133. Edlund MJ, Austen MA, Sullivan MD, Martin BC, Williams JS, Fortney JC, Hudson TJ. Patterns of opioid use for chronic noncancer pain in the Veterans Health Administration from 2009 to 2011. Pain 2014; 155:2337-2343.

134. Dobscha SK, Morasco BJ, Duckart JP, Macey T, Deyo RA. Correlates of prescription opioid initiation and long-term opioid use in veterans with persistent pain. Clinical J Pain 2013; 29:102-108.

135. Wu PC, Lang C, Hasson NK, Linder SH, Clark DJ. Opioid use in young veterans. J Opioid Manage 2010; 6:133-139.

136. Department of Veterans Affairs Veterans Health Administration. Pain Management, VHA Directive 2009-053. October $28,2009$. www.va.gov/painmanagement/docs/ vhaogpaindirective.pdf

137. Blake $H$, Leighton $P$, van der Walt $G$, Ravenscroft $A$. Prescribing opioid analgesics for chronic non-malignant pain in general practice - a survey of attitudes and practice. Br J Pain 2015; 9:225-232.

138. U.S. Food and Drug Administration. FDA Drug Safety Communication: FDA strengthens warning that non-aspirin nonsteroidal anti-inflammatory drugs (NSAIDs) can cause heart attacks or strokes. July 9, 2015.

www.fda.gov/Drugs/DrugSafety/ ucm $451800 . h$ tm

139. Gusovsky D. Americans consume vast majority of the world's opioids. CNBC, Wednesday, April 27, 2016.

www.cnbc.com/2016/04/27/americansconsume-almost-all-of-the-global-opioid-supply.html

140. Drug Enforcement Administration, Deparment of Justice. Final Order: Established Aggregate Production Quotas for Schedule I and II Controlled Substances and Assessment of Annual Needs for the List I Chemicals Ephedrine, Pseudoephedrine, and Phenylpropanolamine for 2017. Federal Register, October 5, 2016.

www.federalregister.gov/documents/2016/10/05/2016-23988/established-aggregate-production-quotasfor-schedule-i-and-ii-controlled-substances-and-assessment

141. Cropsey KL, Stevens EN, Valera P, Brendan Clark C, Bulls HW, Nair P, Lane PS. Risk factors for concurrent use of benzodiazepines and opioids among individuals under community corrections supervision. Drug Alcohol Depend 2015; 154:152-157.

142. Jones JD, Mogali S, Comer SD. Polydrug abuse: A review of opioid and benzodiazepine combination use. Addict Biol 2015; 20:784-798.

143. Nielsen S, Lintzeris N, Bruno R, Campbell G, Larance B, Hall W, Hoban B, Cohen ML, Degenhardt L. Benzodiazepine use among chronic pain patients prescribed opioids: Associations with pain, physical and mental health, and health service utilization. Pain Med 2015; 16:356-366.

144. Hawkins EJ, Malte CA, Grossbard JR, Saxon AJ. Prevalence and trends of concurrent opioid analgesic and benzodiazepine use among veterans affairs patients with post-traumatic stress disorder, 2003- 2011. Pain Med 2015; 16:1943-1954.
145. Jann M, Kennedy WK, Lopez G. Benzodiazepines: A major component in unintentional prescription drug overdoses with opioid analgesics. J Pharm Pract 2014; 27:5-16.

146. Gaither JR, Goulet JL, Becker WC, Crystal S, Edelman EJ, Gordon K, Kerns RD, Rimland D, Skanderson M, Justice AC, Fiellin DA. The association between receipt of guideline-concordant longterm opioid therapy and all-cause mortality. J Gen Intern Med 2016; 31:492-501.

147. Sullivan MD. What are we treating with opioid and sedative-hypnotic combination therapy? Pharmacoepidemiol Drug Saf 2015; 24:893-895.

148. Barry DT, Sofuoglu M, Kerns RD, Wiechers IR, Rosenheck RA. Prevalence and correlates of coprescribing anxiolytic medications with extensive prescription opioid use in Veterans Health Administration patients with metastatic cancer. J Opioid Manag 2016; 12:259-268.

149. Paulozzi LJ, Mack KA, Hockenberry JM; Division of Unintentional Injury Prevention, National Center for Injury Prevention and Control, CDC. Vital signs: Variation among States in prescribing of opioid pain relievers and benzodiazepines - United States, 2012. MMWR Morb Mortal Wkly Rep 2014; 63:563-568.

150. Jones CM, Mack KA, Paulozzi LJ. Pharmaceutical overdose deaths, United States, 2010. JAMA 2013; 309:657-659.

151. Calcaterra S, Glanz J, Binswanger IA. National trends in pharmaceutical opioid related overdose deaths compared to other substance related overdose deaths: 1999 - 2009. Drug Alcohol Depend 2013; 131:263-270.

152. FDA News Release. FDA requires strong warnings for opioid analgesics, prescription opioid cough products, and benzodiazepine labeling related to serious risks and death from combined use. August 31, 2016.

www.fda.gov/NewsEvents/Newsroom/ PressAnnouncements/ucm518697.htm

153. Brady KT, McCauley JL, Back SE. Prescription opioid misuse, abuse, and treatment in the United States: An update. Am J Psychiatry 2016; 173:18-26.

154. Center for Behavioral Health Statistics and Quality. (2015). Behavioral health trends in the United States: Results from the 2014 National Survey on Drug Use and Health (HHS Publication No. SMA 15-4927, NSDUH Series H-50).

155. Gladden RM MP, Seth P. Fentanyl Law Enforcement Submissions and Increases in Synthetic Opioid-Involved Over- 
dose Deaths - 27 States, 2013-2014. MMWR Morb Mortal Wkly Rep 2016; 65:837-843.

156. Cuyahoga County Medical Examiner's Office. Heroin \& Fentanyl Related Deaths in Cuyahoga County. Published September 7, 2016.

http://medicalexaminer.cuyahogacounty.us/pdf_medicalexaminer/en-US/August2016-HeroinFentanylUpdate.pdf

157. DEA Strategic Intelligence Section. National Heroin Threat Assessment Summary - Updated. Washington, DC: United States Drug Enforcement Agency. Published June, 2016. https://www.dea.gov/ divisions/hq/2016/hq062716_attach.pdf

158. Bharel M. An Assessment of Opioid-Related Deaths in Massachusetts (2013 - 2014). Boston: Massachusetts Department of Public Health. Published September 15 , 2016.

http://www.mass.gov/eohhs/gov/departments/dph/stop-addiction/chapter55-overdose-assessment.html

159. DEA Philadelphia Field Division. Analysis of Drug-Related Overdose Deaths in Pennsylvania, 2015. Philadelphia United States Drug Enforcement Administration. Published July, 2016. https://www. dea.gov/divisions/phi/2016/phio71216_ attach.pdf

160. Redican KJ, Marek LI, Brock DJP, McCance-Katz EF. Exploring the etiologic factors and dynamics of prescription drug abuse in Southwest Virginia. Health Promot Perspect 2012; 2:153-165.

161. Boscarino JA, Rukstalis MR, Hoffman SN, Han JJ, Erlich PM, Ross S, Gerhard GS, Stewart WF. Prevalence of prescription opioid-use disorder among chronic pain patients: comparison of the DSM5 vs. DSM-4 diagnostic criteria. J Addict Dis 2011; 8:185-194.

162. Degenhardt L, Hall W. Extent of illicit drug use and dependence, and their contribution to the global burden of disease. Lancet 2012; 379:55-70.

163. Manchikanti L, Malla Y, Wargo BW, Fellows B. Comparative evaluation of the accuracy of immunoassay with liquid chromatography tandem mass spectrometry (LC/MS/MS) of urine drug testing (UDT) opioids and illicit drugs in chronic pain patients. Pain Physician 2011; 14:175-187.

164. Manchikanti L, Malla Y, Wargo BW, Fellows B. Comparative evaluation of the accuracy of benzodiazepine testing in chronic pain patients utilizing immunoassay with liquid chromatography tandem mass spectrometry (LC/MS/MS) of urine drug testing. Pain Physician 2011;
14:259-270.

165. Nordmann S, Pradel V, Lapeyre-Mestre $M$, Frauger E, Pauly V, Thirion X, Mallaret $M$, Jouanjus $E$, Micallef J. Doctor shopping reveals geographical variations in opioid abuse. Pain Physician 2013; 16:89-100.

166. Yarborough BJ, Stumbo SP, Janoff SL, Yarborough MT, McCarty D, Chilcoat HD, Coplan PM, Green CA. Understanding opioid overdose characteristics involving prescription and illicit opioids: A mixed methods analysis. Drug Alcohol Depend 2016; 167:49-56.

167. Davis CS, Johnston JE, Pierce MW. Overdose epidemic, prescription monitoring programs, and public health: A review of state laws. Am J Public Health 2015; 105:e9-el1.

168. McCarberg BH. A critical assessment of opioid treatment adherence using urine drug testing in chronic pain management. Postgrad Med 2011; 123:124-131.

169. Hall AJ, Logan JE, Toblin RL Kaplan JA, Kraner JC, Bixler D, Crosby AE, Paulozzi L). Patterns of abuse among unintentional pharmaceutical overdose fatalities. JAMA 2008; 300:2613-2620.

170. Manchikanti L, Pampati V, Damron K. Prevalence of prescription drug abuse and dependency in patients with chronic pain in western Kentucky. J KY Med Assoc 2003; 101:511-517.

171. Manchikanti L, Manchukonda R, Pampati V, Damron KS. Evaluation of abuse of prescription and illicit drugs in chronic pain patients receiving shortacting (hydrocodone) or long-acting (methadone) opioids. Pain Physician 2005; 8:257-261.

172. Manchikanti L, Cash KA, Damron KS, Manchukonda R, Pampati V, McManus CD. Controlled substance abuse and illicit drug use in chronic pain patients: An evaluation of multiple variables. Pain Physician 2006; 9:215-226.

173. Ives TJ, Chelminski PR, Hammett-Stabler CA, Malone RM, Perhac JS, Potisek NM, Shilliday BB, DeWalt DA, Pignone MP. Predictors of opioid misuse in patients with chronic pain: A prospective cohort study. BMC Health Serv Res 2006; 6:46.

174. Manchikanti L, Pampati V, Damron KS, McManus CD. Evaluation of variables in illicit drug use: Does a controlled substance abuse screening tool identify illicit drug use? Pain Physician 2004; 7:71-75.

175. Manchikanti L, Manchukonda R, Damron KS, Brandon D, McManus CD, Cash
KA. Does adherence monitoring reduce controlled substance abuse in chronic pain patients? Pain Physician 2006; 9:57-60.

176. Pollak KA, Stephens LS, Posner KL, Rathmell JP, Fitzgibbon DR, Dutton RP, Michna E, Domino KB. Trends in pain medicine liability. Anesthesiology 2015; 123:1133-1141.

177. Jones CM, Baldwin GT, Manocchio T, White JO, Mack KA. Trends in methadone distribution for pain treatment, methadone diversion, and overdose deaths - United States, 2002 - 2014. MMWR Morb Mortal Wkly Rep 2016; 65:667-671.

178. Fishbain DA, Cole B, Lewis J, Rosomoff $\mathrm{HL}$, Rosomoff RS. What percentage of chronic nonmalignant pain patients exposed to chronic opioid analgesic therapy develop abuse/addiction and/ or aberrant drug-related behaviors? A structured evidence-based review. Pain Med 2008; 9:444-459.

179. Abdel Shaheed C, Maher CG, Williams KA, Day R, McLachlan AJ. Efficacy, tolerability, and dose-dependent effects of opioid analgesics for low back pain: A systematic review and meta-analysis. JAMA Intern Med 2016; 176:958-968.

180. Hale ME, Dvergsten C, Gimbel J. Efficacy and safety of oxymorphone extended release in chronic low back pain: Results of a randomized, double-blind, placeboand active-controlled phase III study. J Pain 2005; 6:21-28.

181. Allan L, Richarz U, Simpson K, Slappendel R. Transdermal fentanyl versus sustained release oral morphine in strongopioid naïve patients with chronic low back pain. Spine (Phila Pa 1976) 2005; 30:2484-2490.

182. Perrot $S$, Krause D, Crozes $P$, Naïm C; GRTF-ZAL-1 Study Group. Efficacy and tolerability of paracetamol/tramadol $(325 \mathrm{mg} / 37.5 \mathrm{mg}$ ) combination treatment compared with tramadol (50 mg) monotherapy in patients with subacute low back pain: A multicenter, randomized, double-blind, parallel-group, 10day treatment study. Clin Ther 2006; 28:1592-1606.

183. Hale M, Khan A, Kutch M, Li S. Oncedaily OROS hydromorphone ER compared with placebo in opioid-tolerant patients with chronic low back pain. Curr Med Res Opin 2010; 26:1505-1518.

184. Katz N, Rauck R, Ahdieh H, Ma T, Gerritsen van der Hoop R, Kerwin R, Podolsky G. A 12-week, randomized, placebo-controlled trial assessing the safety and efficacy of oxymorphone extended release 
for opioid-naive patients with chronic low back pain. Curr Med Res Opin 2007; 23:117-128.

185. Hale ME, Ahdieh H, Ma T, Rauck R; Oxymorphone ER Study Group 1. Efficacy and safety of OPANA ER (oxymorphone extended release) for relief of moderate to severe chronic low back pain in opioid-experienced patients: A 12-week, randomized, double-blind, placebo-controlled study. J Pain 2007; 8:175-184.

186. Chu LF, D'Arcy N, Brady C, Zamora AK, Young CA, Kim JE, Clemenson AM, Angst MS, Clark JD. Analgesic tolerance without demonstrable opioid-induced hyperalgesia: A double-blinded, randomized, placebo-controlled trial of sustained-release morphine for treatment of chronic nonradicular low-back pain. Pain 2012; 153:1583-1592.

187. Schnitzer TJ, Gray WL, Paster RZ, Kamin M. Efficacy of tramadol in treatment of chronic low back pain. J Rheumatol 2000; 27:772-778.

188. Uberall MA, Mueller-Schwefe $\mathrm{GHH}$, Terhaag B. Efficacy and safety of flupirtine modified release for the management of moderate to severe chronic low back pain: Results of SUPREME, a prospective randomized, double-blind, placebo- and active-controlled parallelgroup phase IV study. Curr Med Res Opin 2012; 28:1617-1634.

189. Vorsanger GJ, Xiang J, Gana TJ, Pascual $\mathrm{ML}$, Fleming RR. Extended-release tramadol (tramadol ER) in the treatment of chronic low back pain. J Opioid Manag 2008; 4:87-97.

190. Peloso PM, Fortin L, Beaulieu A, Ka$\min M$, Rosenthal N; Protocol TRPCAN-1 Study Group. Analgesic efficacy and safety of tramadol/acetaminophen combination tablets (Ultracet) in treatment of chronic low back pain: A multicenter, outpatient, randomized, double blind, placebo controlledtrial. J Rheumatol 2004; 31:2454-2463.

191. Ruoff GE, Rosenthal N, Jordan D, Karim R, Kamin M; Protocol CAPSS-112 Study Group. Tramadol/acetaminophen combination tablets for the treatment of chronic lower back pain: A multicenter, randomized, double-blind, placebocontrolled outpatient study. Clin Ther 2003; 25:1123-1141.

192. Schiphorst Preuper HR, Geertzen JH, van Wijhe M, Boonstra AM, Molmans $\mathrm{BH}$, Dijkstra PU, Reneman MF. Do analgesics improve functioning in patients with chronic low back pain? An explorative triple-blinded RCT. Eur Spine J
2014; 23:800-806.

193. Buynak R, Shapiro DY, Okamoto A, Van Hove I, Rauschkolb C, Steup A, Lange B, Lange C, Etropolski M. Efficacy and safety of tapentadol extended release for the management of chronic low back pain: Results of a prospective, randomized, double-blind, placebo- and activecontrolled Phase III study. Expert Opin Pharmacother 2010; 11:1787-1804.

194. Steiner D, Munera C, Hale M, Ripa S, Landau C. Efficacy and safety of buprenorphine transdermal system (BTDS) for chronic moderate to severe low back pain: A randomized, double-blind study. J Pain 2011; 12:1163-1173.

195. Webster LR, Butera PG, Moran LV, Wu $\mathrm{N}$, Burns LH, Friedmann N. Oxytrex minimizes physical dependence while providing effective analgesia: A randomized controlled trial in low back pain. J Pain 2006; 7:937-946.

196. Cloutier C, Taliano J, O'Mahony W, Csanadi M, Cohen G, Sutton I, Sinclair D, Awde M, Henein S, Robinson L, Eisenhoffer J, Piraino PS, Harsanyi Z, Michalko KJ. Controlled-release oxycodone and naloxone in the treatment of chronic low back pain: A placebocontrolled, randomized study. Pain Res Manag 2013; 18:75-82.

197. Gordon A, Rashiq S, Moulin DE, Gordon A, Rashiq S, Moulin DE Buprenorphine transdermal system for opioid therapy in patients with chronic low back pain. Pain Res Manag 2010; 15:169-178.

198. Steiner DJ, Sitar S, Wen W, Sawyerr G, Munera C, Ripa SR, Landau C. Efficacy and safety of the seven-day buprenorphine transdermal system in opioidnaïve patients with moderate to severe chronic low back pain: An enriched, randomized, double-blind, placebo-controlled study. J Pain Symptom Manage 2011; 42:903-917.

199. Rauck RL, Nalamachu S, Wild JE, Walker GS, Robinson CY, Davis CS, Farr SJ. Single-entity hydrocodone extendedrelease capsules in opioid-tolerant subjects with moderate-to-severe chronic low back pain: A randomized doubleblind, placebo-controlled study. Pain Med 2014; 15:975-985.

200. Von Korff M, Saunders K, Thomas Ray G, Boudreau D, Campbell C, Merrill J, Sullivan MD, Rutter CM, Silverberg MJ, Banta-Green C, Weisner C. De facto long-term opioid therapy for noncancer pain. Clin J Pain 2008; 24:521-527.

201. O'Donnell JB, Ekman EF, Spalding WM, Bhadra P, McCabe D, Berger MF. The effectiveness of a weak opioid medication versus a cyclo-oxygenase-2 (COX-2) selective nonsteroidal anti-inflammatory drug in treating flare-up of chronic low back pain: Results from two randomized, double-blind, 6-week studies. J Int Med Res 2009; 37:1789-1802.

202. Gordon A, Callaghan D, Spink D, Cloutier C, Dzongowski P, O'Mahony W, Sinclair D, Rashiq S, Buckley N, Cohen G, Kim J, Boulanger A, Piraino PS, Eisenhoffer J, Harsanyi Z, Darke AC, Michalko $\mathrm{KJ}$. Buprenorphine transdermal system in adults with chronic low back pain: A randomized, double-blind, placebocontrolled crossover study, followed by an open-label extension phase. Clin Ther 2010; 32:844-860.

203. Khoromi S, Cui L, Nackers L, Max MB. Morphine, nortriptyline and their combination vs. placebo in patients with chronic lumbar root pain. Pain 2007; 130:66-75.

204. Chaparro LE, Furlan AD, Deshpande A, Mailis-Gagnon A, Atlas S, Turk DC. Opioids compared with placebo or other treatments for chronic low back pain: An update of the Cochrane Review. Spine (Phila Pa 1976) 2014; 39:556-563.

205. Canadian Agency for Drugs and Technologies in Health. Long-acting opioids for chronic non-cancer pain: A review of the clinical efficacy and safety. August 27, 2015 .

206. Chou R, Turner JA, Devine EB, Hansen RN, Sullivan SD, Blazina I, Dana T, Bougatsos C, Deyo RA. The effectiveness and risks of long-term opioid therapy for chronic pain: A systematic review for a National Institutes of Health Pathways to Prevention Workshop. Ann Intern Med 2015; 162:276-286.

207. Chung JW, Zeng Y, Wong TK. Drug therapy for the treatment of chronic nonspecific low back pain: Systematic review and meta-analysis. Pain Physician 2013; 16:E685-E704.

208. Reinecke H, Weber C, Lange K, Simon M, Stein C, Sorgatz H. Reinecke H, Weber C, Lange K, Simon M, Stein C, Sorgatz $\mathrm{H}$. Analgesic efficacy of opioids in chronic pain: Recent meta-analyses. $\mathrm{Br}$ ] Pharmacol 2015; 172:324-333.

209. Welsch P, Sommer C, Schiltenwolf M, Häuser W. Opioids in chronic noncancer pain: Are opioids superior to nonopioid analgesics? A systematic review and meta-analysis of efficacy, tolerability and safety in randomized head-to-head comparisons of opioids versus nonopioid analgesics of at least four week's duration [in German]. Schmerz 2015; 29:85-95. 
210. Gaskell H, Moore RA, Derry S, Stannard C. Oxycodone for neuropathic pain in adults. Cochrane Database Syst Rev 2016; 7:CDo10692.

211. Chou R, Deyo R, Friedly J, Skelly A, Hashimoto R, Weimer M, Fu R, Dana T, Kraegel P, Griffin J, Grusing S, Brodt E. Rockville (MD): Agency for Healthcare Research and Quality (US); 2016 Feb. Report No.: 16-EHCoO4-EF.

212. Santos J, Alarcão J, Fareleira F, Vaz-Carneiro A, Costa J. Tapentadol for chronic musculoskeletal pain in adults. Cochrane Database Syst Rev 2015; 5:CDoog923.

213. Cepeda MS, Camargo F, Zea C, Valencia L. Tramadol for osteoarthritis: A systematic review and metaanalysis. J Rheumatol 2007; 34:543-555.

214. Fleischmann RM, Caldwell JR, Roth $\mathrm{SH}$, Roth SH, Tesser JRP, Olson W, Kamin M. Tramadol for the treatment of joint pain associated with osteoarthritis: A randomized, double-blinded, placebo-controlled trial. Curr Ther Res 2001; 62:113-128.

215. Silverfield JC, Kamin M, Wu SC, Rosenthal N. Tramadol/acetaminophen combination tablets for the treatment of osteoarthritis flare pain: A multicenter, outpatient, randomized, double-blind, placebo-controlled, parallel-group, addon study. Clin Ther 2002; 24:282-297.

216. Bird HA, Hill J, Stratford ME, Fenn GC, Wright $V$. A double-blind cross-over study comparing the analgesic efficacy of tramadol with pentazocine in patients with osteoarthritis. J Drug Develop Clin Pract 1995; 7:181-188.

217. Schnitzer TJ, Kamin M, Olson WH. Tramadol allows reduction of naproxen dose among patients with naproxen-responsive osteoarthritis pain: A randomized, double-blind, placebo-controlled study. Arthritis Rheum 1999; 42:1370-1377.

218. Bianchi $M$, Broggini $M$, Balzarini $P$, Baratelli E, Ferrario P, Panerai AE, Sacerdote P. Effects of tramadol on synovial fluid concentrations of substance $P$ and interleukin- 6 in patients with knee osteoarthritis: Comparison with paracetamol. Int Immunopharmacol 2003; 3:1901-1908.

219. Babul N, Noveck R, Chipman H, Roth $\mathrm{SH}$, Gana T, Albert K. Efficacy and safety of extended-release, once-daily tramadol in chronic pain: A randomized 12week clinical trial in osteoarthritis of the knee. J Pain Symptom Manage 2004; 28:59-71.

220. Emkey R, Rosenthal N, Wu S-C, Jordan D, Kamin M; CAPSS-114 Study Group.
Efficacy and safety of tramadol/acetaminophen tablets (Ultracet) as add-on therapy for osteoarthritis pain in subjects receiving a COX-2 nonsteroidal antiinflammatory drug: A multicenter, randomized, double-blind, placebocontrolled trial. J Rheumatol 2004; 31:150-156.

221. Wilder-Smith CH, Hill L, Spargo K, Kalla A. Treatment of severe pain from osteoarthritis with slow-release tramadol or dihydrocodeine in combination with NSAID's: A randomised study comparing analgesia, antinociception and gastrointestinal effects. Pain 2001; 91:23-31.

222. Jensen EM, Ginsberg F. Tramadol versus dextropropoxyphene in the treatment of osteoarthritis: A short term doubleblind study. Drug Invest 1994; 8:211-218.

223. Malonne $H$, Coffiner M, Sonet B, Sereno A, Vanderbist F. Efficacy and tolerability of sustained-release tramadol in the treatment of symptomatic osteoarthritis of the hip or knee: A multicenter, randomized, double-blind, placebo-controlled study. Clin Ther 2004; 26:1774-1782.

224. Pavelka K. Treatment of pain in osteoarthritis. Eur J Pain 2000; 4:23-30.

225. Sandoval JA, Furlan AD, Mailis-Gagnon AM. Oral methadone for chronic noncancer pain: A systemic literature review of reasons for administration, prescription patterns, effectiveness, and side effects. Clin J Pain 2005; 21:503-512.

226. Berken GH, Stone MM, Stone SK. Methadone: An effective agent in managing intractable pain as a symptom of psychotic anger. Ann N Y Acad Sci 1982; 398:83-86.

227. Green J, Coyle M. Methadone use in the control of nonmalignant chronic pain. Pain Manage 1989; 2:241-246.

228. Gardner-Nix JS. Oral methadone for managing chronic nonmalignant pain. J Pain Symptom Manage 1996; 11:321-328.

229. Altier $N$, Dion D, Boulanger $A$, Choinière $M$. Successful use of methadone in the treatment of chronic neuropathic pain arising from burn injuries: A case study. Burns 2001; 27:771-775.

230. Clark JD, Elliott J. A case of a methadone-induced movement disorder. Clin J Pain 2001; 17:375-377.

231. Hays $H$, Woodroffe MA. High dosing methadone and a possible relationship to serious cardiac arrhythmias. Pain Res Manage 2001; 6:64.

232. Gardner-Nix J. Opioids causing peripheral edema. J Pain Symptom Manage 2002; 23:453-455.
233. Mak PH, Tsui SL, Ng KF. Long-term therapy of chronic non-malignant pain with potent opioids in an active police officer. Can J Anaesth 2002; 49:575-578.

234. Bergmans L, Snijdelaar DG, Katz J, Crul BJ. Methadone for phantom limb pain. Clin J Pain 2002; 18:203-205.

235. Cruciani RA, Lucier D, Miller-Saultz D, Arbuck DM. Ultra-low dose oral Naltrexone decreases side effects and potentiates the effect of methadone. J Pain Symptom Manage 2003; 25:491-494.

236. Walker PW, Klein D, Kasza L. High dose methadone and ventricular arrhythmias: A report of three cases. Pain 2003; 103:321-324.

237. Robbins L. Daily opioids (methadone) for refractory chronic daily headache. Headache Quart 1996; 7:39-42.

238. Robbins L. Long-acting opioids (methadone) for refractory chronic daily headache: Quality of life assessment. Headache Quart 1997; 8:234-236.

239. Hays $H$, Woodroffe MA. Use of methadone in treating chronic noncancer pain. Pain Res Manage 1998; 4:23-27.

240. Quang-Cantagrel ND, Wallace MS, Magnuson SK. Opioid substitution to improve the effectiveness of chronic noncancer pain control: A chart review. Anesth Analg 2000; 90:933-937.

241. Hampf GB, Kalso EA. Chronic use of opioids in intractable facial pain. A case report. Acta Odontol Scand 1991; 49:215-218.

242. Moulin DE, Palma D, Watling C, Schultz V. Methadone in the management of intractable neuropathic pain. Pain Res Manag 2003; 8:51B.

243. Mironer YE, Haasis JC III, Somerville JJ, Chapple IT, Barsanti JM. Successful use of methadone in neuropathic pain: A multicenter study by the National Forum of Independent Pain Clinicians. Pain Digest 1999; 9:191-193.

244. Morley JS, Bridson J, Nash TP, Miles JB, White S, Makin MK. Low-dose methadone has an analgesic effect in neuropathic pain: A double-blind randomized controlled crossover trial. Palliat Med 2003; 17:576-87.

245. McNicol ED, Midbari A, Eisenberg E. Opioids for neuropathic pain. Cochrane Database Syst Rev 2013; 8:CDoo6146.

246. Eisenberg E, McNicol E, Carr DB. Opioids for neuropathic pain. Cochrane Database Syst Rev 2006; 3:CDoo6146.

247. Attal N, Guirimand F, Brasseur L, Gaude V, Chauvin M, Bouhassira D. Effects of IV morphine in central pain: A random- 
ized placebo-controlled study. Neurology 2002; 58:554-563.

248. Dellemijn PL, Vanneste JA. Randomised double-blind active-placebo-controlled crossover trial of intravenous fentanyl in neuropathic pain. Lancet 1997; 349:753-758.

249. Eide PK, Jorum E, Stubhaug A, Bremnes J, Breivik $\mathrm{H}$. Relief of post-herpetic neuralgia with the $\mathrm{N}$-methyl-Daspartic acid receptor antagonist ketamine: A doubleblind, cross-over comparison with morphine and placebo. Pain 1994; 58:347-354.

250. Eide PK, Stubhaug A, Stenehjem AE. Central dysesthesia pain after traumatic spinal cord injury is dependent on Nmethyl-D-aspartate receptor activation. Neurosurgery 1995; 37:1080-1087.

251. Frank B, Serpell MG, Hughes J, Matthews JN, Kapur D. Comparison of analgesic effects and patient tolerability of nabilone and dihydrocodeine for chronic neuropathic pain: Randomised, crossover, double blind study. BM] 2008; 336:199-201.

252. Gilron I, Bailey JM, Tu D, Holden RR, Weaver DF, Houlden RL. Morphine, gabapentin, or their combination for neuropathic pain. N Engl JMed 2005; 352:1324-1334.

253. Gimbel JS, Richards P, Portenoy RK. Controlled-release oxycodone for pain in diabetic neuropathy: A randomized controlled trial. Neurology 2003; 60:927-934

254. Jensen MP, Friedman M, Bonzo D, Richards $P$. The validity of the neuropathic pain scale for assessing diabetic neuropathic pain in a clinical trial. Clin J Pain 2006; 22:97-103.

255. Hanna M, O'Brien C, Wilson MC. Prolonged-release Oxycodone enhances the effects of existing Gabapentin therapy in painful diabetic neuropathy patients. Eur J Pain 2008; 12:804-813.

256. Harke H, Gretenkort P, Ladleif HU, Rahman S, Harke O. The response of neuropathic pain and pain in complex regional pain syndrome I to carbamazepine and sustained-release morphine in patients pretreated with spinal cord stimulation: A double-blinded randomized study. Anesth Analg 2001; 92:488-495.

257. Huse E, Larbig W, Flor H, Birbaumer N. The effect of opioids on phantom limb pain and cortical reorganization. Pain 2001; 90:47-55.

258. Jadad AR, Carroll D, Glynn CJ, Moore RA, McQuay HJ. Morphine responsiveness of chronic pain: Doubleblind randomised crossover study with pa- tient-controlled analgesia. Lancet 1992; 339:1367-1371.

259. Jorum E, Warncke T, Stubhaug A. Cold allodynia and hyperalgesia in neuropathic pain: The effect of $\mathrm{N}$-methylDaspartate (NMDA) receptor antagonist ketamine - a doubleblind, cross-over comparison with alfentanil and placebo. Pain 2003; 101:229-235.

26o. Juarez Pichardo JS, Kassian Rank AA, Hernandez Perez AL, Ramirez Tapia Y. Comparison of the efficacy of oxycodone plus lidocaine versus tramadol plus lidocaine in continuous infusion in relieving acute neuropathic pain. Revista de la Sociedad Espanola del Dolor 2009; 16:307-313.

261. Kupers RC, Konings $H$, Adriaensen $H$, Gybels JM. Morphine differentially affects the sensory and affective pain ratings in neurogenic and idiopathic forms of pain. Pain 1991; 47:5-12.

262. Leung A,WallaceMS, Ridgeway B, Yaksh T. Concentration effect relationship of intravenous alfentanil and ketamine on peripheral neurosensory thresholds, allodynia and hyperalgesia of neuropathic pain. Pain 2001; 91:177-187.

263. Max MB, Schafer SC, Culnane M, Dubner R, Gracely RH. Association of pain relief with drug side effects in postherpetic neuralgia: A single-dose study of clonidine, codeine, ibuprofen, and placebo. Clin Pharmacol Ther 1988; 43:363-371.

264. Max MB, Byas-Smith MG, Gracely RH, Bennett GJ. Intravenous infusion of the NMDA antagonist, ketamine, in chronic posttraumatic pain with allodynia: $A$ doubleblind comparison to alfentanil and placebo. Clin Neuropharmacol 1995; 18:360-368.

265. Rabben T, Skjelbred P, Oye I. Prolonged analgesic effect of ketamine, an $\mathrm{N}$ methyl-D-aspartate receptor inhibitor, in patients with chronic pain. J Pharmacol Exp Ther 1999; 289:1060-1066.

266. Arner S, Meyerson BA. Lack of analgesic effect of opioids on neuropathic and idiopathic forms of pain. Pain 1988; 33:11-23.

267. Raja SN, Haythornthwaite JA, Pappagallo M, Clark MR, Travison TG, Sabeen S, Royall RM, Max MB. Opioids versus antidepressants in postherpetic neuralgia: A randomized, placebo-controlled trial. Neurology 2002; 59:1015-1021.

268. Rowbotham MC, Reisner-Keller LA, Fields HL. Both intravenous lidocaine and morphine reduce the pain of postherpetic neuralgia. Neurology 1991;
41:1024-1028.

269. Rowbotham MC, Twilling L, Davies PS, Reisner L, Taylor K, Mohr D. Oral opioid therapy for chronic peripheral and central neuropathic pain. New Engl J Med 2003; 348:1223-1232.

270. Simpson DM, Messina J, Xie F, Hale $M$. Fentanyl buccal tablet for the relief of breakthrough pain in opioid-tolerant adult patients with chronic neuropathic pain: A multicenter, randomized, double-blind, placebo-controlled study. Clin Ther 2007; 29:588-601.

271. Wallace MS, Moulin D, Clark AJ, Wasserman R, Neale A, Morley-Forster P, Castaigne JP, Teichman S. A Phase II, multicenter, randomized, double-blind, placebo-controlled crossover study of CJC-1008 - a long-acting, parenteral opioid analgesic - In the treatment of postherpetic neuralgia. J Opioid Manag 2006; 2:167-173.

272. Watson CP, Babul N. Efficacy of oxycodone in neuropathic pain: A randomized trial in postherpetic neuralgia. Neurology 1998; 50:1837-1841.

273. Watson CP, Moulin D, Watt-Watson J, Gordan A, Eisenhoffer J. Controlledrelease oxycodone relieves neuropathic pain: A randomized controlled trial in painful diabetic neuropathy. Pain 2003; 105:71-78.

274. Wu CL, Tella P, Staats PS, Vaslav R, Kazim DA,Wesselmann U, Raja SN. Analgesic effects of intravenous lidocaine and morphine on postamputation pain: A randomized double-blind, active placebo-controlled, crossover trial. Anesthesiology 2002; 96:841-848.

275. Wu CL, Agarwal S, Tella PK, Klick B, Clark MR, Haythornthwaite JA, Max MB, Raja SN. Morphine versus mexiletine for treatment of postamputation pain: A randomized, placebocontrolled, crossover trial. Anesthesiology 2008; 109:289-296.

276. Zin CS, Nissen LM, O'Callaghan JP, Duffull SB, Smith MT, Moore BJ. A randomized, controlled trial of oxycodone versus placebo in patients with postherpetic neuralgia and painful diabetic neuropathy treated with pregabalin. J Pain 2010; 11:462-471.

277. van Ojik AL, Jansen PA, Brouwers JR, van Roon EN. Treatment of chronic pain in older people: Evidence-based choice of strong-acting opioids. Drugs Aging 2012; 29:615-625.

278. Griessinger N, Sittl R, Likar R. Transdermal buprenorphine in clinical practice: A post-marketing surveillance study in 
13179 patients. Curr Med Res Opin 2005; 21:1147-1156.

279. Gianni W, Madaio AR, Ceci M, Benincasa E, Conati G, Franchi F, Galetti G Nieddu A, Salani B, Zuccaro SM. Transdermal buprenorphine for the treatment of chronic noncancer pain in the oldest old. J Pain Symptom Manage 2011; 41:707-714.

280. Krashin DL, Merrill JO, Trescot AM. Opioids in the management of HIVrelated pain. Pain Physician 2012; 15:ES159-ES198.

281. Eriksen J, Sjogren P, Bruera E, Ekholm $\mathrm{O}$, Rasmussen N.K. Critical issues on opioids in chronic non-cancer pain: An epidemiological study. Pain 2006; 125:172-179.

282. Valkanoff TA, Kline-Simon AH, Sterling S, Campbell C, Von Korff M. Functional disability among chronic pain patients receiving long-term opioid treatment. J Soc Work Disabil Rehabil 2012; 11:128-142.

283. Soin A, Cheng J, Brown L, Moufawad S, Mekhail N. Functional outcomes in patients with chronic nonmalignant pain on long-term opioid therapy. Pain Pract 2008; 8:379-384.

284. Radcliff K, Freedman M, Hilibrand A, Isaac R, Lurie JD, Zhao W, Vaccaro A, Albert T, Weinstein JN. Does opioid pain medication use affect the outcome of patients with lumbar disc herniation? Spine (Phila Pa 1976) 2013; 38:E849-E860.

285. Kidner CL, Mayer TG, Gatchel RJ. Higher opioid doses predict poorer functional outcome in patients with chronic disabling occupational musculoskeletal disorders. J Bone Joint Surg Am 2009; 91:919-927.

286. Franklin GM, Stover BD, Turner JA, Fulton-Kehoe D, Wickizer TM. Early opioid prescription and subsequent disability among workers with back injuries: The Disability Risk Identification Study Cohort. Spine (Phila Pa 1976) 2008; 33:199-204.

287. Franklin GM, Rahman EA, Turner JA, Daniell WE, Fulton-Kehoe D. Opioid use for chronic low back pain: A prospective, population-based study among injured workers in Washington state, 2002 2005. Clin J Pain 2009; 25:743-751.

288. Webster BS, Verma SK, Gatchel RJ. Relationship between early opioid prescribing for acute occupational low back pain and disability duration, medical costs, subsequent surgery, and late opioid use. Spine (Phila Pa 1976) 2007; 32:2127-2132.

289. Ashworth J, Green DJ, Dunn KM, Jor- dan KP. Opioid use among low back pain patients in primary care: Is opioid prescription associated with disability at 6-month follow-up? Pain 2013; 154:1038-1044.

290. Gross DP, Stephens B, Bhambhani Y, Haykowsky M, Bostick GP, Rashiq S. Opioid prescriptions in Canadian workers' compensation claimants: Prescription trends and associations between early prescription and future recovery. Spine (Phila Pa 1976) 2009; 34:525-531.

291. Sites BD, Beach ML, Davis M. Increases in the use of prescription opioid analgesics and the lack of improvement in disability metrics among users. Reg Anesth Pain Med 2014; 39:6-12

292. Faour M, Anderson JT, Ahn NU, Haas AR, Percy R, Woods ST, Ahn UM. Prolonged preoperative opioid therapy associated with poor return to work rates after single-level cervical fusion for radiculopathy for patients receiving workers' compensation benefits. Spine (Phila Pa 1976) 2016 Jul 8 [Epub ahead of print].

293. Burton AK, McClune TD, Clarke RD, Main CJ. Long-term follow-up of patients with low back pain attending for manipulative care: Outcomes and predictors. Man Ther 2004; 9:30-35

294. Chou R, Shekelle P. Will this patient develop persistent disabling low back pain? JAMA 2010; 303:1295-1302.

295. Fanciullo GJ, Ball PA, Girault G, Rose RJ, Hanscom B, Weinstein JN. An observational study on the prevalence and pattern of opioid use in 25,479 patients with spine and radicular pain. Spine (Phila Pa 1976) 2002; 27:201-205.

296. Turk DC, Okifuji A. What factors affect physicians' decisions to prescribe opioids for chronic noncancer pain patients? Clin J Pain 1997; 13:330-336.

297. Bostick GP, Toth C, Carr EC, Stitt LW, Morley-Forster P, Clark AJ, Lynch M, Gordon A, Nathan H, Smyth C, Ware MA, Moulin DE. Physical functioning and opioid use in patients with neuropathic pain. Pain Med 2015; 16:1361-1368.

298. Candiotti KA, Gitlin MC. Review of the effect of opioid-related side effects on the undertreatment of moderate to severe chronic non-cancer pain: Tapentadol, a step toward a solution? Curr Med Res Opin 2010; 26:1677-1684.

299. Jonsson T, Christrup LL, Højsted J, Villesen $\mathrm{HH}$, Albjerg TH, Ravn-Nielsen LV, Sjøgren P. Symptoms and side effects in chronic non-cancer pain: Patient report vs. systematic assessment. Acta Anaes- thesiol Scand 2010; 55:69-74.

300. Furlan AD, Sandoval JA, Mailis-Gagnon A, Tunks E. Opioids for chronic noncancer pain: A meta-analysis of effectiveness and side effects. Can Med Assoc J 2006; 174:1589-1594.

301. Kalso E, Edwards JE, Moore RA, McQuay HJ. Opioids in chronic non-cancer pain: Systematic review of efficacy and safety. Pain 2004; 112:372-380.

302. Moore RA, McQuay H. Prevalence of opioid adverse events in chronic nonmalignant pain: Systematic review of randomised trials of oral opioids. Arthritis Res Ther 2005; 7:R1046-R1051.

303. Lee M, Silverman SM, Hansen H, Patel VB, Manchikanti L. A comprehensive review of opioid-induced hyperalgesia. Pain Physician 2011; 14:145-161.

304. Daniell HW. DHEAS deficiency during consumption of sustained-action prescribed opioids: Evidence for opioidinduced inhibition of adrenal androgen production. J Pain 2006; 7:901-907.

305. Daniell HW. Hypogonadism in men consuming sustained - action oral opioids. J Pain 2002; 3:377-384.

306. Daniell HW. Opioid endocrinopathy in women consuming prescribed sustained-action opioids for control of nonmalignant pain. J Pain 2008; 9:28-36.

307. Smith HS, Elliott J. Opioid-inducted androgen deficiency (OPIAD). Pain Physician 2012; 15:ES145-ES156.

308. Daniell HW. Does methadone prolong QTc intervals by depleting testosterone levels? Arch Intern Med 2010; 170:1407-1408.

309. Daniell HW. Opioid contribution to decreased cortisol levels in critical care patients. Arch Surg 2008; 143:1147-1148.

310. Daniell HW, Lentz R, Mazer NA. Openlabel pilot study of testosterone patch therapy in men with opioid-induced androgen deficiency. J Pain 2006; 7:200-210.

311. Daniell HW. Opioid-induced androgen deficiency discussion in opioid contracts. Am J Med 2007; 120:e21.

312. Scherrer JF, Salas J, Sullivan MD, Schneider FD, Bucholz KK, Burroughs T, Copeland L, Ahmedani B, Lustman PJ. The influence of prescription opioid use duration and dose on development of treatment resistant depression. Prev Med 2016; 91:110-116.

313. Deyo RA, Smith DH, Johnson ES, Tillotson C), Donovan M, Yang X, Petrik A, Morasco BJ, Dobscha SK. Prescription opioids for back pain and use of medi- 
cations for erectile dysfunction. Spine (Phila Pa 1976) 2013; 38:909-915.

314. National Opioids Use Guideline Group (NOUGG). Canadian guidelines for safe and effective use of opioids for chronic non-cancer pain, Recommendations for Practice, Version 5.6. April 30, 2010.

http://nationalpaincentre.mcmaster.ca/ documents/opioid_guideline_part_b_ v5_6.pdf

315. National Institute on Drug Abuse. Overdose death rates.

www.drugabuse.gov/related-topics/ trends-statistics/overdose-death-rates

316. Dunn KM, Saunders KW, Rutter CM, Banta-Green CJ, Merrill JO, Sullivan MD, Weisner CM, Silverberg MJ, Campbell $\mathrm{Cl}$, Psaty BM, Von Korff M. Opioid prescriptions for chronic pain and overdose: A cohort study. Ann Intern Med. 2010; 152:85-92.

317. Bohnert AS, Valenstein $M$, Bair MJ, Ganoczy D, McCarthy JF, Ilgen MA, Blow FC. Association between opioid prescribing patterns and opioid overdoserelated deaths. JAMA 2011; 305:1315-1321.

318. Dasgupta N, Funk MJ, Proescholdbell S, Hirsch A, Ribisl KM, Marshall S. Cohort study of the impact of high-dose opioid analgesics on overdose mortality. Pain Med 2016; 17:85-98.

319. Liang Y, Turner BJ. Assessing risk for drug overdose in a national cohort: Role for both daily and total opioid dose? J Pain 2015; 16:318-325.

320. Zedler B, Xie L,Wang L, Joyce A, Vick C, Brigham J, Kariburyo F, Baser O, Murrelle L. Risk factors for serious prescription opioid-related toxicity or overdose among Veterans Health Administration patients. Pain Med 2014; 15:1911-1929.

321. Bohnert AS, Logan JE, Ganoczy D, Dowell D. A detailed exploration into the association of prescribed opioid dosage and overdose deaths among patients with chronic pain. Med Care 2016; 54:435-441.

322. Gomes T, Mamdani MM, Dhalla IA, Paterson JM, Juurlink DN. Opioid dose and drug-related mortality in patients with nonmalignant pain. Arch Intern Med 2011; 171:686-691.

323. Paulozzi LJ, Kilbourne EM, Shah NG, Nolte KB, Desai HA, Landen MG, Harvey $\mathrm{W}$, Loring $\mathrm{LD}$. A history of being prescribed controlled substances and risk of drug overdose death. Pain Med 2012; 13:87-95.

324. Gaither JR, Leventhal JM, Ryan SA, Camenga DR. National trends in hospitalizations for opioid poisonings among children and adolescents, 1997 to 2012. JAMA Pediatr 2016; 170:1195-1201.

325. Paulozzi L, Mack KA, Jones CM; Centers for Disease Control and Prevention (CDC). Vital signs: Risk for overdose frommethadone used for pain relief: United States, $1999-2010$. MMWR Morb Mortal Wkly Rep 2012; 61:493-497.

326. Jones CM, McAninch JK. Emergency department visits and overdose deaths from combined use of opioids and benzodiazepines. Am J PrevMed 2015; 49:493-501.

327. Webster LR, Choi Y, Desai H, Webster L, Grant BJB. Sleep-disordered breathing and chronic opioid therapy. Pain Med 2008; 9:425-432.

328. U.S. Department of Veterans Affairs. VA/DoD clinical practice guidelines: Management of opioid therapy (OT) for chronic pain (2010). US Department of Veterans Affairs, Department of Defense.

www.healthquality.va.gov/guidelines/ Pain/cot/

329. Brunton LL, ed. Goodman and Gilman's the Pharmacologic Basis of Therapeutics. gth ed. McGraw-Hill, New York, 1996.

330. Rolita L, Spegman A, Tang X, Cronstein BN. Greater number of narcotic analgesic prescriptions for osteoarthritis is associated with falls and fractures in elderly adults. J AmGeriatr Soc 2013; 61:335-340.

331. Spector W, Shaffer T, Potter DE, Correade-Araujo R, Rhona Limcangco M. Risk factors associated with the occurrence of fractures in US nursing homes: Resident and facility characteristics and prescription medications. J Am Geriatr Soc 2007; 55:327-333.

332. Yazdy MM, Mitchell AA, Tinker SC, Parker SE, Werler MM. Periconceptional use of opioids and the risk of neural tube defects. Obstet Gynecol 2013; 122:838-844.

333. Whiteman VE, Salemi JL, Mogos MF, CainMA, Aliyu MH, Salihu HM. Maternal opioid drug use during pregnancy and its impact on perinatal morbidity, mortality, and the costs of medical care in the United States. J Pregnancy 2014; 2014:906723.

334. Hadi I, da Silva O, Natale R, Boyd D, Morley-Forster PK. Opioids in the parturient with chronic nonmalignant pain: A retrospective review. J Opioid Manag 2006; 2:31-34.

335. Edlund MJ, Steffick D, Hudson T, Harris KM, Sullivan M. Risk factors for clinically recognized opioid abuse and dependence among veterans using opioids for chronic non-cancer pain. Pain 2007; 129:355-362.

336. Reid MC, Engles-Horton LL, Weber MB, Kerns RD, Rogers EL, O'Connor PG. Use of opioid medications for chronic noncancer pain syndromes in primary care. J Gen Intern Med 2002; 17:173-179.

337. Howe CQ, Sullivan MD. The missing "P" in pain management: How the current opioid epidemic highlights the need for psychiatric services in chronic pain care. Gen Hosp Psychiatry 2014; 36:99-104.

338. Office of National Drug Control Policy. How illicit drug use affects business and the economy.

www.whitehouse.gov/ondcp/ondcpfact-sheets/how-illicit-drug-use-affectsbusiness-and-the-economy

339. National Institute on Drug Abuse. Prescription opioids and heroin.

www.drugabuse.gov/publications/research-reports/relationship-betweenprescription-drug-abuse-heroin-use/ introduction

340. Cicero TJ, Ellis MS, Harney J. Shifting patterns of prescription opioid and heroin abuse in the United States. N Engl J Med 2015; 373:1789-1790.

341. Compton WM, Jones CM, Baldwin GT. Relationship between nonmedical prescription-opioid use and heroin use. $N$ Engl] Med 2016; 374:154-163.

342. Wilder CM, Miller SC, Tiffany E, Winhusen T, Winstanley EL, Stein MD. Risk factors for opioid overdose and awareness of overdose risk among veterans prescribed chronic opioids for addiction or pain. J Addict Dis 2016; 35:42-51.

343. Volkow ND. America's addiction to opioids: Heroin and prescription drug abuse. National Institute on Drug Abuse, May 14, 2014.

www.drugabuse.gov/about-nida/legislative-activities/testimony-to-congress/2015/americas-addiction-to-opioids-heroin-prescription-drug-abuse

344. Shah NG, Lathrop SL, Reichard RR, Landen MG. Unintentional drug overdose death trends in New Mexico, USA, 1990 - 2005: Combinations of heroin, cocaine, prescription opioids and alcohol. Addiction 2008; 103:126-136.

345. Martins SS, Sampson L, Cerdá M, Galea S. Worldwide prevalence and trends in unintentional drug overdose: A systematic review of the literature. Am J Public Health 2015; 105:e29-e49.

346. Albert M, McCaig LF, Uddin S. Emergency department visits for drug poisoning: United States, 2008-2011. NCHS Data Brief 2015; 196:1-8. 
347. Hedegaard $H$, Chen LH, Warner M. Drug-poisoning deaths involving heroin: United States, $2000-2013$. NCHS Data Brief 2015; 190:1-8.

348. Centers for Disease Control and Prevention (CDC). CDC Vital Signs. Opioid painkiller prescribing. Where you live makes a difference. July 2014 .

www.cd c.gov/vitalsigns/ opioid-prescribing/

349. Lev R, Petro S, Lee A, Lee O, Lucas J, Castillo EM, Egnatios J, Vilke GM. Methadone related deaths compared to all prescription related deaths. Forensic Sci Int 2015; 257:347-352.

350. Weimer MB, Korthuis PT, Behonick GS, Wunsch MJ. The source of methadone in overdose deaths in Western Virginia in 2004. J Addict Med 2011; 5:188-202.

351. Methadone Mortality, Center for Substance Abuse Treatment, Washington, DC, 2010, Substance Abuse and Mental Health Services Administration.

352. Chou R, Cruciani RA, Fiellin DA, Compton $P$, Farrar JT, Haigney MC, Inturrisi $C$, Knight JR, Otis-Green $S$, Marcus SM, Mehta D, Meyer MC, Portenoy R, Savage S, Strain E, Walsh S, Zeltzer L; American Pain Society; Heart Rhythm Society. Methadone safety: A clinical practice guideline from the American Pain Society and College on Problems of Drug Dependence, in collaboration with the Heart Rhythm Society. J Pain 2014; 15:321-337.

353. Weimer MB, Chou R. Research gaps on methadone harms and comparative harms: Findings from a review of the evidence for an American Pain Society and College on Problems of Drug Dependence clinical practice guideline. J Pain 2014; 15:366-376.

354. Larochelle MR, Liebschutz JM, Zhang F, Ross-Degnan D, Wharam JF. Opioid prescribing after nonfatal overdose and association with repeated overdose: A cohort study opioid prescribing after nonfatal overdose. Ann Intern Med 2016; 164:1-9.

355. Leung SY. Benzodiazepines, opioids and driving: An overview of the experimental research. Drug Alcohol Rev 2011; 30:281-286.

356. Fishbain DA, Cutler RB, Rosomoff HL, Rosomoff RS. Are opioid-dependent/ tolerant patients impaired in driving-related skills? A structured evidence-based review. J Pain Symptom Manage 2003; 25:559-577.

357. Wilhelmi BG, Cohen SP. A framework for "driving under the influence of drugs" policy for the opioid using driver.
Pain Physician 2012; 15:ES215-ES230.

358. Miceli L, Bednarova R, Sandri M, Rizzardo A, Rocca GD. Use of opioids for pain relief while driving: When the patient meets the police. Pain Pract 2013; 13:345.

359. Sabatowski R, Mordenti G, Miceli L. Opioids and driving ability: Current data do not support one opioid being more favorable than another. Pain Pract 2014; 14:196-197.

360. Sabatowski R. Driving ability under opioids: Current assessment of published studies. Dtsch Med Wochenschr 2008; 133:S25-S28.

361. Miceli L, Bednarova R, Rizzardo A, Marcassa C, Della Rocca G. Alcohol, pain, and opioids: Which is a major threat to driving ability? Ann Pharmacother 2014; 48:1531-1132.

362. Kelly E, Darke S, Ross J. A review of drug use and driving: Epidemiology, impairment, risk factors and risk perceptions. Drug Alcohol Rev 2004; 23:319-344.

363. Lenne MG, Dietze P, Rumbold G, Redman JR, Triggs TJ. Opioid dependence and driving ability: A review in the context of proposed legislative change in Victoria. Drug Alcohol Rev 2000; 19:427-439.

364. Berghaus G, Friedel B. Methadone and driver fitness. Euro-Methwork Newsletter February 1998; 13 .

365. Walsh MJ. A State-by-State Analysis of Laws Dealing with Driving Under the Influence of Drugs. United States Department of Transportation. National Highway Traffic Safety Administration (NHSTA), 2009.

366. Manchikanti L, Atluri S, Kaye AM, Kaye AD. Hydrocodone bitartrate for chronic pain. Drugs Today 2015; 51:415-427.

367. Califf RM, Woodcock J, Ostroff S. A proactive response to prescription opioid abuse. N Engl J Med 2016; 374:1480-1485.

368. Fischer B, Keates A, Bühringer $G$, Reimer J, Rehm J. Nonmedical use of prescription opioids and prescription opioidrelated harms: Why so markedly higher in North America compared to the rest of the world? Addiction 2014; 109:177-181.

369. Centers for Disease Control and Prevention (CDC). (2014) CDC's Top Ten: 5 Health Achievements in 2013 and 5 Health Threats in 2014. Atlanta, GA: CDC.

https://blogs.cdc.gov/cdcworksforyou 24-7/2013/12/cdc\%E2\%80\%99s-topten-5-health-achievements-in-2013and-5-health-threats-in-2014/

370. National Center for Health Statistics.
Percent of drug poisoning deaths that mention the type of drug(s) involved, by state: 2013-2014. Atlanta: Centers for Disease Control and Prevention.

http://www.cdc.gov/nchs/data/ health_policy/unspecified_drugs_by_ state_2013-2014.pdf.

371. Express Scripts Lab. A nation in pain: Focusing on U.S. opioid trends for treatment of short-term and longer term pain. An Express Scripts Report, December 9, 2014 .

372. Straus MM, Ghitza UE, Tai B. Preventing deaths from rising opioid overdose in the US - the promise of naloxone antidote in community-based naloxone take-home programs. Subst Abuse Rehabil 2013; 2013:65-72.

373. Cantrill SV, Brown MD, Carlisle RJ, Delaney KA, Hays DP, Nelson LS, O'Connor RE, Papa A, Sporer KA, Todd KH, Whitson RR; American College of Emergency Physicians Opioid Guideline Writing Panel. Clinical policy: Critical issues in the prescribing of opioids for adult patients in the emergency department. Ann Emerg Med 2012; 60:499-525.

374. Mehendale AW, Goldman MP, Mehendale RP. Opioid overuse pain syndrome (OOPS): The story of opioids, Prometheus unbound. J Opioid Manag 2013; 9:421-438.

375. Davis JM, Severtson SG, Bucher-Bartelson B, Dart RC. Using poison center exposure calls to predict prescription opioid abuse and misuse-related emergency department visits. Pharmacoepidemiol Drug Saf 2014; 23:18-25.

376. National Association of Medicaid Directors. State medicaid interventions for preventing prescription drug abuse and overdose: A report for the national association of Medicaid directors. October 1 , 2014.

www.integration.samhsa.gov/namd_rx_ abuse_report_october_2014.pdf

377. Letter to Janet Woodcock, MD, Director, Center for Drug Evaluation and Research, US FDA, from Physicians for Responsible Opioid Prescribing RE Docket No. FDA-2011-D- 0771, Draft Blueprint for Prescriber Education for Long-Acting/Extended Release Opioid ClassWide Risk Evaluation and Mitigation Strategies. December 2, 2011.

www.supportprop.org/wp-content/uploads/2014/12/PA_12_2_11_REMS_Blueprint.pdf

378. Volkow ND. What is the federal government doing to combat the opioid abuse epidemic? Natl Inst Drug Abuse May 1, 2015 . 
www.drugabuse.gov/about-nida/legislative-activities/testimony-to-congress/2016/what-federal-governmentdoing-to-combat-opioid-abuse-epidemic

379. Toomey, Kaine Call on GAO To Review Pain Clinics' Narcotics Dispensing Practices: Gov't Watchdog Would Also Examine Quality of Treatment, Patient Outcomes. Washington, D.C. December 23, 2015.

380. Volkow ND, McLellan TA. Curtailing diversion and abuse of opioid analgesics without jeopardizing pain treatment. JAMA 2011; 305:1346-1347.

381. Strassels SA. Economic burden of prescription opioid misuse and abuse. J Manag Care Pharm 2009; 15:556-562.

382. Manchikanti L, Boswell MV, Hirsch JA. Lessons learned in the abuse of painrelief medication: A focus on healthcare costs. Expert Rev Neurother 2013; 13:527-544.

383. Garcia AM. State laws regulating prescribing of controlled substances: Balancing the public health problems of chronic pain and prescription painkiller abuse and overdose. J Med Ethics 2013; 41:42-45.

384. Ballantyne JC, Sullivan MD, Kolodny A. Opioid dependence vs addiction: A distinction without a difference? Arch Intern Med 2012; 172:1342-1343.

385. Patrick SW, Fry CE, Jones TF, Buntin MB. Implementation of prescription drug monitoring programs associated with reductions in opioid-related death rates. Health Aff (Millwood) 2016; 35:1324-1332.

386. Bachhuber MA, Maughan BC, Mitra N, Feingold J, Starrels JL. Prescription monitoring programs and emergency department visits involving benzodiazepine misuse: Early evidence from in United States metropolitan areas. Int J Drug Policy 2016; 28:120-123.

387. Sheperd J. Combating the prescription painkiller epidemic: A national prescription drug reporting program. Am J Law Med 2014; 40:85-112.

388. Griggs CA, Weiner SG, Feldman JA. Prescription drug monitoring programs: Examining limitations and future approaches. West ] Emerg Med 2015; 16:67-70.

389. Islam MM, McRae IS. An inevitable wave of prescription drug monitoring programs in the context of prescription opioids: Pros, cons and tensions. BMC Pharmacol Toxicol 2014; 15:46.

390. Starrels JL, Becker WC, Alford DP, Kapoor A, Williams AR, Turner BJ. System- atic review: Treatment agreements and urine drug testing to reduce opioid misuse in patients with chronic pain. Ann Intern Med 2010; 152:712-720.

391. Nguyen V, Raffa RB, Taylor R, Pergolizzi $J V$. The role of abuse deterrent formulations in countering opioid misuse and abuse. J Clin Pharm Ther 2015; 40:629-634.

392. Leece $P$, Orkin AM, Kahan M. Tamperresistant drugs cannot solve the opioid crisis. CMA] 2015; 187:717-718.

393. Larochelle MR, Zhang F, Ross-Degnan $D$, Wharam JF. Rates of opioid dispensing and overdose after introduction of abuse deterrent extended-release oxycodone and withdrawal of propoxyphene. JAMA Intern Med 2015; 175:978-987.

394. Clark AK, Wilder CM, Winstanley EL. A systematic review of community opioid overdose prevention and naloxone distribution programs. J Addict Med 2014; 8:153-163.

395. Conermann T, Gosalia AR, Kabazie AJ, Moore C, Miller K, Fetsch M, Irvan D. Utility of oral fluid in compliance monitoring of opioid medications. Pain Physician 2014; 17:63-70.

396. Fudin J. The perfect storm: Opioid risks and "The Holy Trinity." Pharmacy Times, September 24, 2014.

www.pharmacytimes.com/contributor/jeffrey-fudin/2014/og/the-perfectstorm-opioid-risks-and-the-holy-trinity

397. Courtwright DT. Preventing and treating narcotic addiction - century of federal drug control. N Engl J Med 2015; 373:2095-2097.

398. Turner JA, Saunders K, Shortreed SM, Rapp SE, Thielke S, LeResche L, Riddell KM, Von Korff M. Chronic opioid therapy risk reduction initiative: Impact on urine drug testing rates and results. J Gen Intern Med 2014; 29:305-311.

399. Bird SM, McAuley A, Perry S, Hunter C. Effectiveness of Scotland's national naloxone programme for reducing opioid related deaths: A before (2006-10) versus after (2011-13) comparison. Addiction 2016; 111:883-891.

400. Imtiaz S, Shield KD, Fischer B, Rehm J. Harms of prescription opioid use in the United States. Subst Abuse Treat Prev Policy 2014; 9:43.

401. Gasior M, Bond M, Malamut R. Routes of abuse of prescription opioid analgesics: A review and assessment of the potential impact of abuse-deterrent formulations. Postgrad Med 2016; 128:85-96.

402. Physicians for Responsible Opioid Pre- scribing (PROP).

www.supportprop.org/

403. O'Kane N, Hallvik SE, Marino M, Van Otterloo J, Hildebran C, Leichtling G, Deyo RA. Preparing a prescription drug monitoring program data set for research purposes. Pharmacoepidemiol Drug Saf 2016; 25:993-997.

404. Chang HY, Lyapustina T, Rutkow L, Daubresse M, Richey M, Faul M, Stuart EA, Alexander GC. Impact of prescription drug monitoring programs and pill mill laws on high-risk opioid prescribers: A comparative interrupted time series analysis. Drug Alcohol Depend 2016; 165:1-8.

405. Sankey C, Setnik B, Harsanyi Z, Michalko K, Yang Z, Geoffroy P. Opioid use following the introduction of an extended-release oxycodone formulation with tamper-resistant properties: Prospective historical chart review in methadonemaintained patients. J Opioid Manag 2016; 12:149-159.

406. Coplan PM, Chilcoat HD, Butler SF, Sellers EM, Kadakia A, Harikrishnan V, Haddox JD, Dart RC. The effect of an abuse-deterrent opioid formulation (OxyContin) on opioid abuse-related outcomes in the post-marketing setting. Clin Pharmacol Ther 2016; 100:275-286.

407. Meara E, Horwitz JR, Powell W, McClelland L, Zhou W, O'Malley AJ, Morden NE. State legal restrictions and prescription-opioid use among disabled adults. N Engl J Med 2016; 375:44-53.

408. Dowell D, Zhang K, Noonan RK, Hockenberry JM. Mandatory provider review and pain clinic laws reduce the amounts of opioids prescribed and overdose death rates. Health Aff (Millwood) 2016; 35:1876-1883.

409. Manchikanti L, Pampati V, Hirsch JA. Utilization of interventional techniques in managing chronic pain in Medicare population from 2000 to 2014: An analysis of patterns of utilization. Pain Physician 2016; 19: E531-E546.

410. Kentucky All Schedules Prescription Electronic Reporting Act (KASPER) Advisory Council.

www.chfs.ky.gov/os/oig/kasperadvisorycouncil.htm

411. American Board of Interventional Pain Physicians.

www.abipp.org/

412. Cappola AR, FitzGerald GA. Confluence, not conflict of interest: Name change necessary. JAMA 2015; 314:1791-1792.

413. Manchikanti L, Benyamin RM, Falco FJE, Caraway DL, Datta S, Hirsch JA. 
Guidelines warfare over interventional techniques: Is there a lack of discourse or straw man? Pain Physician 2012; 15:E1-E26.

414. Manchikanti L, Knezevic NN, Boswell MV, Kaye AD, Hirsch JA. Epidural injections for lumbar radiculopathy and spinal stenosis: A comparative systematic review and meta-analysis. Pain Physician 2016; 19:E365-E410.

415. Manchikanti L, Kaye AD, Hirsch JA. Comment RE: Chou R, Hashimoto R, Friedly J, et al RE: Epidural corticosteroid injections for radiculopathy and spinal stenosis: A systematic review and metaanalysis. Ann Intern Med 2015; 163:373381; Ann Intern Med 2016; 164:633.

416. Boswell MV, Manchikanti L. Appropriate design and methodologic quality assessment, clinically relevant outcomes are essential to determine the role of epidural corticosteroid injections. Commentary RE: Chou R, Hashimoto R, Friedly J, Fu R, Bougatsos C, Dana $\mathrm{T}$, Sullivan SD, Jarvik J. Epidural corticosteroid injections for radiculopathy and spinal stenosis: A systematic review and meta-analysis. Ann Intern Med 2015; 163:373-381. Evid Based Med 2016; 21:89.

417. Krasselt $M$, Häuser W, Petzke F, Baerwald C. S3 guidelines on long-term opioid treatment in non-cancer pain: Recommendations for opioid use in clinical rheumatology. Z Rheumatol 2016; 75:128-132.

418. Alford DP. Opioid prescribing for chronic pain - Achieving the right balance through education. N Engl J Med 2016; 374:301-303.

419. U.S. Food and Drug Administration. Risk Evaluation and Mitigation Strategy (REMS) for Extended-Release and LongActing Opioids.

www.fda.gov/drugs/drugsafety/informationbydrugclass/ucmi63647.htm

420. Office of Inspector General. Report (OEI04-11-00510): FDA Lacks Comprehensive Data To Determine Whether Risk Evaluation and Mitigation Strategies Improve Drug Safety. February 12, 2013.

https://oig.hhs.gov/oei/reports/OEI-O411-00510.asp

421. Hwang CS, Turner LW, Kruszewski SP, Kolodny A, Alexander GC. Primary care physicians' knowledge and attitudes regarding prescription opioid abuse and diversion. Clin J Pain 2016; 32:279-284.

422. Hupp JR. The surgeon's roles in stemming the prescription opioid abuse epidemic. J Oral Maxillofac Surg 2016; 16:1-3.
423. Mudumbai SC, Oliva EM, Lewis ET, Trafton J, Posner D, Mariano ER, Stafford RS, Wagner T, Clark JD. Time-tocessation of postoperative opioids: A population-level analysis of the Veterans Affairs Health Care System. Pain Med 2016; 17:1732-1743.

424. Gold LS, Strassels SA, Hansen RN. Health care costs and utilization in patients receiving prescriptions for longacting opioids for acute postsurgical pain. Clin J Pain 2016; 32:747-754.

425. Minkowitz HS, Gruschkus S, Shah M, Raju A. Adverse drug events among patients receiving postsurgical opioids in a large health system: Risk factors and outcomes. Am J Health Syst Pharm 2014; 71:1556-1565.

426. American College of Surgeons launches education program on opioids and surgery: Use, abuse, and alternatives. Pain Medicine News, November 3, 2016.

www.painmedicinenews.com/WebOnly/Article/11-16/American-Collegeof-Surgeons-Launches-Education-Program-on-Opioids-and-Surgery/38455

427. del Portal DA, Healy ME, Satz WA, McNamara RM. Impact of an opioid prescribing guideline in the acute care setting. J Emerg Med 2016; 50:21-27.

428. Kirschner N, Ginsburg J, Sulmasy LS. Prescription drug abuse: A policy position paper from the American College of Physicians. Ann Intern Med 2014; 160:198.

429. Thielke SM, Turner JA, Shortreed SM, Saunders K, Leresche L, Campbell CI, Weisner CC, Korff MV. Do patient-perceived pros and cons of opioids predict sustained higher-dose use? Clin J Pain 2014; 30:93-101.

430. Hooten WM, St. Sauver JL, McGree ME, Jacobson DJ, Warner DO. Incidence and risk factors for progression from shortterm to episodic or long-term opioid prescribing: A population-based study. Mayo Clin Proc 2015; 90:850-856.

431. Helmerhorst GT, Vranceanu AM, Vrahas $M$, Smith $M$, Ring D. Risk factors for continued opioid use one to two months after surgery for musculoskeletal trauma. J Bone Joint Surg Am 2014; 96:495-499.

432. Rozet I, Nishio I, Robbertze R, Rotter D, Chansky H, Hernandez AV. Prolonged opioid use after knee arthroscopy in military veterans. Anesth Analg 2014; 119:454-459.

433. Krebs EE, Lurie JD, Fanciullo G, Tosteson TD, Blood EA, Carey TS, Weinstein JN. Predictors of long-term opioid use among patients with painful lumbar spine conditions. J Pain 2010; 11:44-52.

434. Goesling J, Moser SE, Zaidi B, Hassett A, Hilliard Paul, Hallstrom B, Clauw DJ, Brummett CM. Trends and predictors of opioid use after total knee and total hip arthroplasty. Pain 2016; 157:1259-1265.

435. Thielke S, Shortreed SM, Saunders K, Turner JA, LeResche L, von Korff M. A prospective study of predictors of longterm opioid use among patients with chronic non-cancer pain. Clin J Pain 2016 [Epub ahead of print].

436. Bateman BT, Franklin JM, Bykov K, Avorn J, Shrank WH, Brennan TA, Landon JE, Rathmell JP, Huybrechts KF, Fischer MA, Choudhry NK. Persistent opioid use following cesarean delivery: Patterns and predictors among opioidnaïve women. Am J Obstet Gynecol 2016; 215:353.e1-353.e18.

437. Rutkow L, Chang HY, Daubresse M, Webster DW, Stuart EA, Alexander GC. Effect of Florida's prescription drug monitoring program and pill mill laws on opioid prescribing and use. JAMA Intern Med 2015; 175:1642-1649.

438. Johnson H, Paulozzi L, Porucznik C, Mack K, Herter B; Hal Johnson Consulting and Division of Disease Control and Health Promotion, Florida Department of Health. Decline in drug overdose deaths after state policy changes - Florida, 2010 - 2012. MMWR Morb Mortal Wkly Rep 2014; 63:569-574.

439. Haegerich TM, Paulozzi LJ, Manns BJ, Jones CM. What we know, and don't know, about the impact of state policy and systems-level interventions on prescription drug overdose. Drug Alcohol Depend 2014; 145:34-47.

440. Bair MJ, Krebs EE.Why is urine drug testing not used more often in practice? Pain Pract 2010; 10:493-496.

441. Walley AY, Xuan Z, Hackman HH, Quinn E, Doe-Simkins M, Sorensen-Alawad A, Ruiz S, Ozonoff A. Opioid overdose rates and implementation of overdose education and nasal naloxone distribution in Massachusetts: Interrupted time series analysis. BM] 2013; 346:f174.

442. Kaye $A D$, Jones MR, Kaye AM, Ripoll JG, Galan V, Beakley BD, Calixto F, Vo N, Bolden JL, Urman RD, Manchikanti L. Prescription opioid abuse in chronic pain: An updated review of opioid abuse predictors and strategies to curb opioid abuse: Part 1. Pain Physician 2017; 20:S93-S110.

443. Kaye AD, Jones MR, Ripoll JG, Jones DE, Galan V, Beakley BD, Calixto F, Urman 
RD, Manchikanti L. Prescription opioid abuse in chronic pain: An updated review of opioid abuse predictors and strategies to curb opioid abuse (Part 2). Pain Physician 2017; 20:S111-S134.

444. Centers for Disease Control and Prevention. Injury Prevention \& Control: Prescription Drug Overdose.

www.cdc.gov/drugoverdose/policy/successes.html

445. Lyapustina T, Rutkow L, Chang HY, Daubresse M, Ramji AF, Faul M, Stuart EA, Alexander GC. Effect of a "pill mill" law on opioid prescribing and utilization: The case of Texas. Drug Alcohol Depend 2016; 159:190-197.

446. Jena $A B$, Goldman D, Karaca-Mandic P. Hospital prescribing of opioids to Medicare beneficiaries. JAMA Intern Med 2016; 176:990-997.

447. Jiang $X$, Orton $M$, Feng R, Hossain $E$, Malhotra NR, Zager EL, Liu R. Chronic opioid usage in surgical patients in a large academic center. Ann Surg 2016 [Epub ahead of print].

448. Public law No: 109-60. H.R.1132, National All Schedules Prescription Electronic Reporting Act of 2005, signed by President George W. Bush on 8/11/05.

www.congress.gov/109/plaws/publ6o/ PLAW-109publ6o.pdf

449. Harold Rogers Prescription Drug Monitoring Program. U.S. Department of Justice.

www.bja.gov/ProgramDetails. aspx?Program_ID=72

450. U.S. General Accounting Office. GAO04-524T. Prescription Drugs, State Monitoring Programs May Help to Reduce Illegal Diversion. May 4, 2004. www.gao.gov/assets/120/110650.pdf

451. National Alliance for Model State Drug Laws (NAMSDL): 2015 Annual Review of Prescription Monitoring Programs, September 2015 www.namsdl.org/library/1810E284AoD7-D440-C3A9A0560A1115D7/

452. Centers for Disease Control and Prevention. Clues to opioid abuse from state prescription drug monitoring programs, October 15, 2015.

www.cdc.gov/media/releases/2015/ p1015-opioid-abuse.html

453. Appleby J. Opioid problems bring 'tsunami' of services, study finds. MedPage Today, August 1, 2016.

www.medpagetoday.com/psychiatry/ addictions/59437

454. Chou R, Fanciullo GJ, Fine PG, Mias- kowski C, Passik SD, Portenoy RK. Opioids for chronic noncancer pain: Prediction and identification of aberrant drug-related behaviors: A review of the evidence for an American Pain Society and American Academy of Pain Medicine clinical practice guideline. J Pain 2009; 10:131-146.

455. The opioid crisis among the privately insured. The opioid abuse epidemic as documented in private claims data. A FAIR Health White Paper. Fair Health, Inc. July 2016.

www.fairhealth.org/servlet/servlet.FileD ownload?file $=01532000001 \mathrm{nWD}_{2}$

456. Manchikanti L, Manchukonda R, Pampati V, Damron KS, Brandon DE, Cash KA, McManus CD. Does random urine drug testing reduce illicit drug use in chronic pain patients receiving opioids? Pain Physician 2006; 9:123-129.

457. Christo PJ, Manchikanti L, Ruan X, Bottros $M$, Hansen $H$, Solanki D, Jordan $A E$, Colson J. Urine drug testing in chronic pain. Pain Physician 2011; 14:123-143.

458. Solanki DR, Koyyalagunta D, Shah RV, Silverman SM, Manchikanti L. Monitoring opioid adherence in chronic pain patients: Assessment of risk of substance misuse. Pain Physician 2011; 14:E119-E131.

459. Jones CM, Muhuri PK, Lurie PG. Trends in the nonmedical use of OxyContin, United States, 2006 - 2013. Clin J Pain 2016 Aug 10 [Epub ahead of print].

460. Maincent J, Zhang F. Recent advances in abuse-deterrent technologies for the delivery of opioids. Int J Pharm 2016; 510:57-72.

461. Busch AJ, Barber KAR, Overend TJ, Peloso PMJ, Schachter CL. Exercise for treating fibromyalgia syndrome. Cochrane Database Syst Rev 2007; 4:CDo03786.

462. Fransen M, McConnell S, Harmer AR, Van der Esch $M$, Simic $M$, Bennell $\mathrm{KL}$. Exercise for osteoarthritis of the knee. Cochrane Database Syst Rev 2015; 1:CDoo4376.

463. Fransen M, McConnell S, HernandezMolina G, Reichenbach S. Exercise for osteoarthritis of the hip. Cochrane Database Syst Rev 2014; 4:CDoo7912.

464. Hayden JA, van Tulder MW, Malmivaara A, Koes BW. Exercise therapy for treatment of non-specific low back pain. Cochrane Database Syst Rev 2005; 3:CDooo335.

465. Saragiotto BT, Maher CG, Yamato TP, Costa LO, Menezes Costa LC, Ostelo RW, Macedo LG. Motor control exercise for chronic non-specific low-back pain. Cochrane Database Syst Rev 2016; 1:CDo12004.

466. Collins SL, Moore RA, McQuay HJ, Wiffen P. Antidepressants and anticonvulsants for diabetic neuropathy and postherpetic neuralgia: A quantitative systematic review. J Pain Symptom Manage 2000; 20:449-458.

467. Lee C, Crawford C, Swann S; Active SelfCare Therapies for Pain (PACT) Working Group. Multimodal, integrative therapies for the self-management of chronic pain symptoms. Pain Med 2014; 15:S76-S85.

468. Lunn MP, Hughes RA, Wiffen PJ. Duloxetine for treating painful neuropathy, chronic pain or fibromyalgia. Cochrane Database Syst Rev 2014; 1:CDoo7115.

469. Moore RA, Straube S, Wiffen PJ, Derry $\mathrm{S}, \mathrm{McQuay} \mathrm{HJ}$. Pregabalin for acute and chronic pain in adults. Cochrane Database Syst Rev 2009; 8:CDoo7076.

470. Moore RA, Wiffen PJ, Derry S, Toelle T, Rice AS. Gabapentin for chronic neuropathic pain and fibromyalgia in adults. Cochrane Database Syst Rev 2014; 4:CDoo7938.

471. Roelofs PD, Deyo RA, Koes BW, Scholten RJ, van Tulder MW. Nonsteroidal antiinflammatory drugs for low back pain: An updated Cochrane review. Spine (Phila Pa 1976) 2008; 33:1766-1774.

472. Chou R, Qaseem A, Snow V, Casey D, Cross JT Jr, Shekelle P, Owens DK; Clinical Efficacy Assessment Subcommittee of the American College of Physicians; American College of Physicians; American Pain Society Low Back Pain Guidelines Panel. Diagnosis and treatment of low back pain: A joint clinical practice guideline from the American College of Physicians and the American Pain Society. Ann Intern Med 2007; 147:478-491.

473. Saarto T, Wiffen PJ. Antidepressants for neuropathic pain: A Cochrane review. J Neurol Neurosurg Psychiatry 2010; 81:1372-1373.

474. Salerno SM, Browning R, Jackson JL. The effect of antidepressant treatment on chronic back pain: A meta-analysis. Arch Intern Med 2002; 162:19-24.

475. Staiger TO, Gaster B, Sullivan MD, Deyo RA. Systematic review of antidepressants in the treatment of chronic low back pain. Spine (Phila Pa 1976) 2003; 28:2540-2545.

476. Trelle S, Reichenbach S, Wandel S, Hildebrand P, Tschannen B, Villiger PM, Egger M, Jüni P. Cardiovascular safety of non-steroidal anti-inflammatory drugs: Network meta-analysis. BMJ 2011; 
342:c7086.

477. Williams AC, Eccleston C, Morley S. Psychological therapies for the management of chronic pain (excluding headache) in adults. Cochrane Database Syst Rev 2012; 11:CDoo7407.

478. Wiffen PJ, Derry S, Moore RA, Kalso EA. Carbamazepine for chronic neuropathic pain and fibromyalgia in adults. Cochrane Database Syst Rev 2014; 4:CDoo5451.

479. Abdel Shaheed C, Maher CG, Williams KA, McLachlan AJ. Efficacy and tolerability of muscle relaxants for low back pain: Systematic review and meta-analysis. Eur] Pain 2016 [Epub ahead of print].

480. Bavage S, Durg S, Kareem SA, Dhadde SB. Clinical efficacy and safety of eperisone for low back pain: A systematic literature review. Pharmacol Rep 2016; 68:903-912.

481. Enthoven WT, Roelofs PD, Deyo RA, van Tulder MW, Koes BW. Non-steroidal anti-inflammatory drugs for chronic low back pain. Cochrane Database Syst Rev 2016; 2:CDo12087.

482. Kamper SJ, Apeldoorn AT, Chiarotto A, Smeets RJ, Ostelo RW, Guzman J, van Tulder MW. Multidisciplinary biopsychosocial rehabilitation for chronic low back pain. Cochrane Database Syst Rev 2014; 9:CDooog63.

483. Kroon FP, van der Burg LR, Ramiro S, Landewé RB, Buchbinder R, Falzon L, van der Heijde D. Non-steroidal antiinflammatory drugs (NSAIDs) for axial spondyloarthritis (ankylosing spondylitis and non-radiographic axial spondyloarthritis). Cochrane Database Syst Rev 2015; 7:CDo10952.

484. Machado GC, Maher CG, Ferreira PH, Pinheiro MB, Lin CW, Day RO, McLachIan AJ, Ferreira ML. Efficacy and safety of paracetamol for spinal pain and osteoarthritis: Systematic review and meta-analysis of randomised placebo controlled trials. BM] 2015; 350:h1225.

485. Häuser W, Urrútia G, Tort S, Uçeyler N, Walitt B. Serotonin and noradrenaline reuptake inhibitors (SNRIs) for fibromyalgia syndrome. Cochrane Database Syst Rev 2013; 1:CDo10292.

486. Xing RL, Wang PM. Bisphosphonates therapy for osteoarthritis: A meta-analysis of randomized controlled trials. Springerplus 2016; 5:1704.

487. Kaye AD, Manchikanti L, Abdi S, Atluri $\mathrm{S}$, Bakshi S, Benyamin R, Boswell MV, Buenaventura R, Candido KD, Cordner HJ, Datta S, Doulatram G, Gharibo CG, Grami V, Gupta S, Jha S, Kaplan ED, Malla Y, Mann DP, Nampiaparampil
DE, Racz G, Raj P, Rana MV, Sharma $M L$, Singh V, Soin A, Staats PS, Vallejo R, Wargo BW, Hirsch JA. Efficacy of epidural injections in managing chronic spinal pain: A best evidence synthesis. Pain Physician 2015; 18:E939-E1004.

488. Manchikanti L, Benyamin RM, Falco FJ, Kaye AD, Hirsch JA. Do epidural injections provide short- and long-term relief for lumbar disc herniation? A systematic review. Clin Orthop Relat Res 2015; 473:1940-1956.

489. Manchikanti L, Nampiaparampil DE, Manchikanti KN, Falco FJE, Singh V, Benyamin RM, Kaye AD, Sehgal N, Soin A, Simopoulos TT, Bakshi S, Gharibo CG, Gilligan CJ, Hirsch JA. Comparison of the efficacy of saline, local anesthetics, and steroids in epidural and facet joint injections for the management of spinal pain: A systematic review of randomized controlled trials. Surg Neurol Int 2015; 6:S194-S235.

490. Manchikanti L, Kaye AD, Manchikanti KN, Boswell MV, Pampati V, Hirsch JA. Efficacy of epidural injections in the treatment of lumbar central spinal stenosis: A systematic review. Anesth Pain Med 2015; 5:e23139.

491. Manchikanti L, Nampiaparampil DE, Candido KD, Bakshi S, Grider JS, Falco FJE, Sehgal N, Hirsch JA. Do cervical epidural injections provide long-term relief in neck and upper extremity pain? A systematic review. Pain Physician 2015; 18:39-60.

492. Chang Chien GC, Knezevic NN, McCormick Z, Chu SK, Trescot AM, Candido KD. Transforaminal versus interlaminar approaches to epidural steroid injections: A systematic review of comparative studies for lumbosacral radicular pain. Pain Physician 2014; 17:E509-E524.

493. Manchikanti L, Singh V, Pampati V, Falco FJ, Hirsch JA. Comparison of the efficacy of caudal, interlaminar, and transforaminal epidural injections in managing lumbar disc herniation: Is one method superior to the other? Korean ] Pain 2015; 28:11-21.

494. Manchikanti L, Staats PS, Nampiaparampil DE, Hirsch JA. What is the role of epidural injections in the treatment of lumbar discogenic pain: A systematic review of comparative analysis with fusion and disc arthroplasty. Korean J Pain 2015; 28:75-87.

495. Liu K, Liu P, Liu R, Wu X, Cai M. Steroid for epidural injection in spinal stenosis: A systematic review and meta-analysis. Drug Des Devel Ther 2015; 9:707-716.

496. Macvicar J, King W, Landers MH, Bog- duk N. The effectiveness of lumbar transforaminal injection of steroids: A comprehensive review with systematic analysis of the published data. Pain Med 2013; 14:14-28.

497. Bicket M, Gupta A, Brown CH, Cohen SP. Epidural injections for spinal pain: A systematic review and meta-analysis evaluating the "control" injections in randomized controlled trials. Anesthesiology 2013; 119:907-931.

498. Cohen SP, Bicket MC, Jamison D, Wilkinson I, Rathmell JP. Epidural steroids: A comprehensive, evidencebased review. Reg Anesth Pain Med 2013; 38:175-200.

499. Helm II S, Racz GB, Gerdesmeyer L, Justiz L, Hayek SM, Kaplan ED, El Terany MA, Knezevic NN. Percutaneous and endoscopic adhesiolysis in managing low back and lower extremity pain: A systematic review and meta-analysis. Pain Physician 2016; 19:E245-E282.

500. Manchikanti L, Manchikanti KN, Gharibo CG, Kaye AD. Efficacy of percutaneous adhesiolysis in the treatment of lumbar post surgery syndrome. Anesth Pain Med 2016; 6:e26172.

501. Boswell MV, Manchikanti L, Kaye AD, Bakshi S, Gharibo CG, Gupta S, Jha S, Nampiaparampil DE, Simopoulos TT, Hirsch JA. A best-evidence systematic appraisal of the diagnostic accuracy and utility of facet (zygapophysial) joint injections in chronic spinal pain. Pain Physician 2015; 18: $E_{497-E_{533} .}$

502. Manchikanti L, Kaye AD, Boswell MV, Bakshi S, Gharibo CG, Grami V, Grider JS, Gupta S, Jha S, Mann DP, Nampiaparampil DE, Sharma ML, Shroyer LN, Singh V, Soin A, Vallejo R, Wargo BW, Hirsch JA. A systematic review and best evidence synthesis of the effectiveness of therapeutic facet joint interventions in managing chronic spinal pain. Pain Physician 2015; 18: E535-E582.

503. Manchikanti L, Hirsch JA, Falco FJ, Boswell MV. Management of lumbar zygapophysial (facet) joint pain. World J Orthop 2016; 7:315-337.

504. Manchikanti L, Hirsch JA, Kaye AD, Boswell MV. Cervical zygapophysial (facet) joint pain: Effectiveness of interventional management strategies. Postgrad Med 2016; 128:54-68.

505. Simopoulos TT, Manchikanti L, Gupta S, Aydin SM, Kim CH, Solanki D, Nampiaparampil DE, Singh V, Staats PS, Hirsch JA. Systematic review of the diagnostic accuracy and therapeutic effectiveness of sacroiliac joint interventions. Pain Physician 2015; 18:E713-E756. 
506. Grider JS, Manchikanti L, Carayannopoulos A, Sharma ML, Balog CC, Harned ME, Grami V, Justiz R, Nouri KH, Hayek SM, Vallejo R, Christo PJ. Effectiveness of spinal cord stimulation in chronic spinal pain: A systematic review. Pain Physician 2016; 19:E33-E54.

507. Pinto RZ, Maher CG, Ferreira ML, Hancock M, Oliveira VC, McLachlan AJ, Koes $\mathrm{B}$, Ferreira PH. Epidural corticosteroid injections in the management of sciatica: A systematic review and meta-analysis. Ann Intern Med 2012; 157:865-77.

508. Chou R, Hashimoto R, Friedly J, Fu Rochelle, Dana T, Sullivan S, Bougatsos C, Jarvik J. Pain Management Injection Therapies for Low Back Pain. Technology Assessment Report ESIBo813. (Prepared by the Pacific Northwest Evidence-based Practice Center under Contract No. HHSA 290-2012-00014I.) Rockville, MD: Agency for Healthcare Research and Quality; July 10, 2015.

509. Staal JB, de Bie RA, de Vet HC, Hildebrandt J, Nelemans P. Injection therapy for subacute and chronic low back pain: An updated Cochrane review. Spine (Phila Pa 1976) 2009; 34:49-59.

510. Chou R, Hashimoto R, Friedly J, Fu R, Bougatsos C, Dana T, Sullivan SD, Jarvik J. Epidural corticosteroid injections for radiculopathy and spinal stenosis: A systematic review and meta-analysis. Ann Intern Med 2015; 163:373-381.

511. O'Connor AB, Dworkin RH. Treatment of neuropathic pain: An overview of recent guidelines. Am J Med 2009; 122:S22-S32.

512. Attal N, Cruccu G, Baron R Haanpää $M$, Hansson P, Jensen TS, Nurmikko T; European Federation of Neurological Societies. EFNS guidelines on the pharmacological treatment of neuropathic pain: 2010 revision. Eur J Neurol 2010; 17:1113-e88.

513. Eden J, Levit L, Berg A, Morton S (eds); Committee on Standards for Systematic Reviews of Comparative Effectiveness Research; Institute of Medicine. Finding What Works in Health Care. Standards for Systematic Reviews. The National Academies Press, Washington, DC, 2011.

514. Moulin DE, Clark AJ, Gilron I, Ware MA, Watson CP, Sessle BJ, Coderre T, Morley-Forster PK, Stinson J, Boulanger A, Peng $P$, Finley GA, Taenzer $P$, Squire $P$, Dion D, Cholkan A, Gilani A, Gordon A, Henry J, Jovey R, Lynch M, Mailis-Gagnon A, Panju A, Rollman GB, Velly A; Canadian Pain Society. Pharmacological management of chronic neuropathic pain: Consensus statement and guidelines from the Canadian Pain Society. Pain Res Manag 2007; 12:13-21.

515. FDA Drug Safety Communication: FDA strengthens warning that nonaspirin nonsteroidal anti-inflammatory drugs (NSAIDs) can cause heart attacks or strokes. US Food and Drug Administration.

www.fda.gov/Drugs/DrugSafety/ ucm45180o.htm

516. U.S. Food and Drug Administration Acetaminophen Information.

www.fda.gov/Drugs/DrugSafety/InformationbyDrugClass/ucm165107.htm

517. U.S. Food and Drug Administration Nonsteroidal Anti-inflammatory Drugs (NSAIDs).

www.fda.gov/Drugs/DrugSafety/PostmarketDrugSafetylnformationforPatientsandProviders/ucm103420.htm

518. Moore A, Wiffen P, Kalso E. Antiepileptic drugs for neuropathic pain and fibromyalgia. JAMA 2014; 312:182-183.

519. Manchikanti L. CBT for low-back pain in primary care. Lancet 2010; 375:869-870.

520. Lewis RA, Williams N, Matar HE, Din $N$, Fitzsimmons D, Phillips $C$, Jones $M$, Sutton A, Burton K, Nafees S, Hendry M, Rickard I, Chakraverty R, Wilkinson $C$. The clinical effectiveness and costeffectiveness of management strategies for sciatica: Systematic review and economic model. Health Technol Assess 2011; 15:1-578.

521. Lewis RA, Williams NH, Sutton AJ, Burton K, Din NU, Matar HE, Hendry M, Phillips CJ, Nafees S, Fitzsimmons D, Rickard I, Wilkinson C. Comparative clinical effectiveness of management strategies for sciatica: Systematic review and network meta-analyses. Spine J 2015; 15:1461-1477.

522. British Pain Society and Faculty of Pain Medicine of the Royal College of Anaesthetists. Standard of good practice for spinal interventional procedures in pain medicine. April 2015.

https://www.britishpainsociety.org/static/uploads/resources/files/spinal_intervention_A5_Final_April_2015_1.pdf

523. Kemler MA, Furnee CA. Economic evaluation of spinal cord stimulation for chronic reflex sympathetic dystrophy. Neurology 2002; 59:1203-1209.

524. North RB, Kidd D, Shipley J, Taylor RS. Spinal cord stimulation versus reoperation for failed back surgery syndrome: A cost effectiveness and cost utility analysis based on a randomized, controlled trial. Neurosurgery 2007; 61:361-368.
525. Kumar K, Taylor RS, Jacques L, Eldabe S, Meglio M, Molet J, Thomson S, O'Callaghan J, Eisenberg E, Milbouw $G$, Buchser E, Fortini G, Richardson J, North RB. The effects of spinal cord stimulation in neuropathic pain are sustained: A 24-month follow-up of the prospective randomized controlled multicenter trial of the effectiveness of spinal cord stimulation. Neurosurgery 2008; 63:762-770.

526. Kapural L, Yu C, Doust MW, Gliner BE, Vallejo R, Sitzman BT, Amirdelfan K, Morgan DM, Yearwood TL, Bundschu R, Yang T, Benyamin R, Burgher AH. Comparison of $10-\mathrm{kHz}$ high-frequency and traditional low-frequency spinal cord stimulation for the treatment of chronic back and leg pain: 24-month results from a multicenter, randomized, controlled pivotal trial. Neurosurgery 2016; 79:667-677.

527. Al-Kaisy A, Van Buyten JP, Smet I, Palmisani S, Pang D, Smith T. Sustained effectiveness of $10 \mathrm{kHz}$ high-frequency spinal cord stimulation for patients with chronic, low back pain: 24-month results of a prospective multicenter study. Pain Med 2014; 15:347-454.

528. Hou S, Kemp K, Grabois M. A systematic evaluation of burst spinal cord stimulation for chronic back and limb pain. Neuromodulation 2016; 19:398-405.

529. Aiyer R, Barkin RL, Bhatia A, Gungor S. A systematic review on the treatment of phantom limb pain with spinal cord stimulation. Pain Manag 2017; 7:59-69.

530. Verrills P, Sinclair C, Barnard A. A review of spinal cord stimulation systems for chronic pain. J Pain Res 2016; 9:481-492.

531. Taylor RS, Ryan J, O'Donnell R, Eldabe S, Kumar K, North RB. The cost-effectiveness of spinal cord stimulation in the treatment of failed back surgery syndrome. Clin J Pain 2010; 26:463-469.

532. Manchikanti L, Helm S 2nd, Pampati V, Racz GB. Cost utility analysis of percutaneous adhesiolysis in managing pain of post-lumbar surgery syndrome and lumbar central spinal stenosis. Pain Pract 2015; 15:414-422.

533. Manchikanti L, Falco FJE, Pampati V, Cash KA, Benyamin RM, Hirsch JA. Cost utility analysis of caudal epidural injections in the treatment of lumbar disc herniation, axial or discogenic low back pain, central spinal stenosis, and post lumbar surgery syndrome. Pain Physician 2013; 16:E129-E143.

534. Moore RA, Derry S, Simon LS, Emery P. Nonsteroidal anti-inflammatory drugs, 
gastroprotection, and benefit-risk. Pain Pract 2014; 14:378-395.

535. Zeidan AZ, Al Sayed B, Bargaoui N Djebbar M, Djennane M, Donald R, El Deeb K, Joudeh RA, Nabhan A, Schug SA. A review of the efficacy, safety, and cost-effectiveness of COX-2 inhibitors for Africa and the Middle East region. Pain Pract 2013; 13:316-331.

536. Xie F, Tanvejsilp P, Campbell K, Gaebel K. Cost-effectiveness of pharmaceutical management for osteoarthritis pain: A systematic review of the literature and recommendations for future economic evaluation. Drugs Aging 2013; 30:277-284.

537. Wielage RC, Bansal M, Andrews JS, Wohlreich MM, Klein RW, Happich M. The cost-effectiveness of duloxetine in chronic low back pain: A US private payer perspective. Value Health 2013; 16:334-344.

538. Bhusal S, Diomampo S, Magrey MN. Clinical utility, safety, and efficacy of pregabalin in the treatment of fibromyalgia. Drug Health Patient Saf 2016; 8:13-23.

539. Choy E, Richards S, Bowrin K, Watson P, Lloyd A, Sadosky A, Zlateva G. Cost effectiveness of pregabalin in the treatment of fibromyalgia from a UK perspective. Curr Med Res Opin 2010; 26:965-975

540. Crofford LJ, Rowbotham MC, Mease PJ, Russell IJ, Dworkin RH, Corbin AE, Young JP Jr, LaMoreaux LK, Martin SA, Sharma U; Pregabalin 1008-105 Study Group. Pregabalin for the treatment of fibromyalgia syndrome: results of a randomized, double-blind, placebocontrolled trial. Arthritis Rheum 2005: 52:1264-1273.

541. Arnold LM, Russell IJ, Diri EW, Duan WR, Young JP Jr, Sharma U, Martin SA, Barrett JA, Haig G. A 14-week, randomized, double-blinded, placebo-controlled monotherapy trial of pregabalin in patients with fibromyalgia. J Pain 2008; 9:792-805.

542. Mease PJ, Russell IJ, Arnold LM, Florian $\mathrm{H}$, Young JP Jr, Martin SA, Sharma U. A randomized, double-blind, placebocontrolled, phase III trial of pregabalin in the treatment of patients with fibromyalgia.J Rheumatol 2008; 35:502-514.

543. Pauer L, Winkelmann $A$, Arsenault $P$, Jespersen A, Whelan L, Atkinson G, Leon T, Zeiher B; Aoo8110o Investigators. An international, randomized, doubleblind, placebo-controlled, phase III trial of pregabalin monotherapy in treatment of patients with fibromyalgia. J Rheuma- tol 2011; 38:2643-2652.

544. Pergolizzi JV Jr, Raffa RB, Zampogna G, Breve F, Colucci R, Schmidt WK, LeQuang JA. Comments and suggestions from pain specialists regarding the CDC's proposed opioid guidelines. Pain Pract 2016; 16:794-808.

545. CDC survey results. Pain News Network

ww w. painnews network. org/ cdc-survey-results/

546. Webster LR. Is suicide a consequence of the CDC opioid guideline? Pain Medicine News, August 11, 2016.

www.painmedicinenews.com/ Commentary/Article/o8-16/IsSuicide-a-Consequence-of-theCDC-Opioid-Guideline-/37442/ ses=ogst

547. Guerriero F, Reid MC. New opioid prescribing guidelines released in the US: What impact will they have in the care of older patients with persistent pain? Curr Med Res Opin 2016 10:1-4.

548. Alliance for Patient Access, American Academy of Pain Management, American Cancer Society, Cancer Action Network, Inc., American Chronic Pain Association, Center for Lawful Access and Abuse Deterrence, Chronic Pain Research Group at the Clinical Pharmacokinetics Laboratory, University at Buffalo School of Pharmacy and Pharmaceutical Sciences, Hospice and Palliative Nurses Association, Interstitial Cystitis Association, Massachusetts Pain Initiative, National Fibromyalgia \& Chronic Pain Association, Pain Connection, Project Lazarus, The Pain Community, US Pain Foundation, Virginia Cancer Pain Initiative. CDC gets it transparently wrong on opioids. DrugWonks.com, September 21, 2015.

http://drugwonks.com/blog/cdc-gets-ittransparently-wrong-on-opioids

549. Johnson SR. New CDC guidelines are unlikely to affect opioid prescribing practices. Mod Healthc 2016; 46:11.

550. Franklin GM, Fulton-Kehoe D, Turner JA, Sullivan MD, Wickizer TM. Changes in opioid prescribing for chronic pain in Washington State. J Am Board Fam Med 2013; 26:394-400.

551. Manchikanti L, Singh V, Pampati V, Boswell MF, Benyamin RM, Hirsch JA. Description of documentation in the management of chronic spinal pain. Pain Physician 2009: 12:E199-E224.

552. Manchikanti L. The role of evaluation and management services in pain management. Pain Physician 1999; 2:10-32.

553. Manchikanti L, Benyamin RM. Docu- mentation for evaluation and management services. In: Manchikanti L, Christo PJ, Trescot AM, Falco FJE (eds). Foundations of Pain Medicine and Interventional Pain Management: A Comprehensive Review. ASIPP Publishing, Paducah, KY, 2011, pp 455-470.

554. Manchikanti L. Evaluation and management in interventional pain practice: Doing it right! Pain Physician 2000; 3:322-341.

555. Manchikanti L, Singh V, Hirsch JA. Documentation for interventional techniques. In: Manchikanti L, Christo PJ, Trescot AM, Falco FJE (eds). Foundations of Pain Medicine and Interventional Pain Management: A Comprehensive Review. ASIPP Publishing, Paducah, KY, 2011, pp 471-486.

556. Manchikanti L. Appropriate documentation, billing and coding of interventional pain procedures. Pain Physician 2000; 3:218-236.

557. Nuwer MR, Sigsbee B. The Health Care Financing Administration's new examination documentation criteria: minimum auditing standards for the neurologic examination to be used by Medicare and other payors. Report from the American Academy of Neurology Medical Economics and Management Subcommittee. Neurology 1998; 50:497-500.

558. Welsby P. Taut teachings: History and physical examination tips for examinations. Postgrad Med J 2016; 92:247-249.

559. Schultz MA, Doty M. Why the history and physical examination still matter. JAAPA 2016; 29:41-45.

560. Spader HS, Grossberg JA, Haas RA, Soares GM. Fundamentals of the neurologic examination for patients undergoing central nervous system interventional procedures. Semin Intervent Radiol 2013; 30:240-244.

561. Manchikanti L, Pampati V, Fellows B, Baha A. The inability of the clinical picture to characterize pain from facet joints. Pain Physician 2000; 3:158-166.

562. Manchikanti L, Hammer MJ, Boswell MV, Kaye AD, Hirsch JA. A seamless navigation to ICD-10-CM for interventional pain physicians: Is a rude awakening avoidable? Pain Physician 2016; 19:E1-E14.

563. Manchikanti L, Hammer M, Benyamin RM, Hirsch JA. Physician Quality Reporting System (PQRS) for interventional pain management practices: Challenges and opportunities. Pain Physician 2016; 19:E15-E32.

564. Davis JA, Robinson RL, Le TK, Xie J. In- 
cidence and impact of pain conditions and comorbid illnesses. J Pain Res 2011; 4:331-345.

565. Leong IY, Farrell MJ, Helme RD, Gibson S). The relationship between medical comorbidity and self-rated pain, mood disturbance, and function in older people with chronic pain. J Gerontol A Biol Sci Med Sci 2007; 62:550-555.

566. Maixner W, Fillingim RB, Williams DA, Smith SB, Slade GD. Overlapping chronic pain conditions: Implications for diagnosis and classification. J Pain 2016; 17:T93-T107.

567. Manchikanti L, Pampati V, Fellows B, Beyer CD, Damron KS, Barnhill RC, Burks T. Characteristics of chronic low back pain in patients in an interventional pain management setting: A prospective evaluation. Pain Physician 2001; 4:131-142.

568. Barry LC, Guo Z, Kerns RD, Duong BD, Reid MC. Functional self-efficacy and pain-related disability among older veterans with chronic pain in a primary care setting. Pain 2003; 104:131-137.

569. Fairbank JC, Pynsent PB. The Oswestry Disability Index. Spine (Phila Pa 1976) 2000; 25:2940- 2952.

570. Cleland JA, Childs JD, Whitman JM. Psychometric properties of the Neck Disability Index and Numeric Pain Rating Scale in patients with mechanical neck pain. Arch Phys Med Rehabil 2008; 89:69-74.

571. Robertson JA, Purple RJ, Cole P, Zaiwalla Z, Wulff K, Pattinson KT. Sleep disturbance in patients taking opioid medication for chronic back pain. Anaesthesia 2016; 71:1296-1307.

572. Cheatle MD, Webster LR. Opioid Therapy and sleep disorders: Risks and mitigation strategies. Pain Med 2015; 16:S22-S26.

573. Correa D, Farney RJ, Chung F, Prasad A, Lam D, Wong J. Chronic opioid use and central sleep apnea: A review of the prevalence, mechanisms, and perioperative considerations. Anesth Analg 2015; 120:1273-1285.

574. Rose AR, Catcheside PG, McEvoy RD, Paul D, Kapur D, Peak E, Vakulin A, Antic NA. Sleep disordered breathing and chronic respiratory failure in $\mathrm{pa}-$ tients with chronic pain on long term opioid therapy. J Clin Sleep Med 2014; 10:847-852.

575. Pampati S, Manchikanti L. What is the prevalence of symptomatic obstructive sleep apnea syndrome in chronic spinal pain patients? An assessment of the correlation of OSAS with chronic opioid therapy, obesity, and smoking. Pain Physician 2016; 19: $\mathrm{E}_{569}-\mathrm{E}_{579}$.

576. Hassamal S, Miotto K, Wang T, Saxon AJ. A narrative review: The effects of opioids on sleep disordered breathing in chronic pain patients and methadone maintained patients. Am J Addict 2016; 25:452-465.

577. Van Ryswyk E, Antic N. Opioids and sleep disordered breathing. Chest 2016; 150:934-944.

578. STOP-Bang Questionnaire - Screening tool for obstructive sleep apnea. www.stopbang.ca/osa/general.php

579. Manchikanti L, Cash KA, Pampati V, Fellows $B$. Influence of psychological variables on the diagnosis of facet joint involvement in chronic spinal pain. Pain Physician 2008; 11:145-160.

580. Manchikanti L, Pampati V, Fellows B, Rivera JJ, Damron KS, Beyer CD, Cash KA. Influence of psychological factors on the ability to diagnose chronic low back pain of facet joint origin. Pain Physician 2001; 4:349-357.

581. Manchikanti L, Giordano J, Boswell MV, Fellows B, Manchukonda R, Pampati V. Psychological factors as predictors of opioid abuse and illicit drug use in chronic pain patients. J Opioid Manag 2007; 3:89-100.

582. Manchikanti L, Pampati V. Research designs in interventional pain management: Is randomization superior, desirable or essential? Pain Physician 2002; 5:275-284.

583. Manchikanti L, Pampati V, Beyer CD, Damron KS. Do number of pain conditions influence emotional status? Pain Physician 2002; 5:200-205.

584. Eccleston C, Morley SJ, Williams AC. Psychological approaches to chronic pain management: Evidence and challenges. Br J Anaesth 2013; 111:59-63.

585. Sparkes E, Duarte RV, Mann S, Lawrence TR, Raphael JH. Analysis of psychological characteristics impacting spinal cord stimulation treatment outcomes: A prospective assessment. Pain Physician 2015; 18:E369-E377.

586. American Psychiatric Association. Diagnostic and Statistical Manual of Mental Disorders. 5th ed. American Psychiatric Association, Washington, DC, 2013.

587. Traeger AC, Hübscher M, Henschke N, Williams CM, Maher CG, Moseley GL, Lee $\mathrm{H}$, McAuley JH. Emotional distress drives health services overuse in patients with acute low back pain: A longitudinal observational study. Eur Spine
J 2016; 25:2767-2773.

588. Heneweer $H$, Aufdemkampe $G$, van Tulder MW, Kiers H, Stappaerts KH, Vanhees L. Psychosocial variables in patients with (sub)acute low back pain: An inception cohort in primary care physical therapy in The Netherlands. Spine (Phila Pa 1976) 2007; 32:586-592.

589. Kothe R, Kohlmann TH, Klink T, Rüther $W$, Klinger R. Impact of low back pain on functional limitations, depressed mood and quality of life in patients with rheumatoid arthritis. Pain 2007; 127:103-108.

590. Keeley P, Creed F, Tomenson B, Todd C, Borglin G, Dickens C. Psychosocial predictors of health-related quality of life and health service utilisation in people with chronic low back pain. Pain 2008; 135:142-150.

591. Hülsebusch J, Hasenbring MI, Rusu AC. Understanding pain and depression in back pain: The role of catastrophizing, help-/hopelessness, and thought suppression as potential mediators. Int ] Behav Med 2016; 23:251-259.

592. Sielski R, Glombiewski JA. Biofeedback as a psychological treatment option for chronic back pain. Int J Behav Med 2016 [Epub ahead of print].

593. Pegram SE, Lumley MA, Jasinski MJ, Burns JW. Psychological trauma exposure and pain-related outcomes among people with chronic low back pain: Moderated mediation by thought suppression and social constraints. Ann Behav Med 2016 [Epub ahead of print].

594. Cherniack M, Dillon C, Erdil M, Ferguson S, Kaplan J, Krompinger J, Litt M, Murphy M. Clinical and psychological correlates of lumbar motion abnormalities in low back disorders. Spine J 2001; 1:290-298.

595. Carrage EJ. Psychological and functional profiles in select subjects with low back pain. Spine J 2001; 1:198-204.

596. Matcham F, Norton S, Scott DL, Steer S, Hotopf M. Symptoms of depression and anxiety predict treatment response and long-term physical health outcomes in rheumatoid arthritis: Secondary analysis of a randomized controlled trial. Rheumatology (Oxford) 2016; 55:268-278.

597. Fama CA, Chen N, Prusik J, Kumar V, Wilock M, Roth S, Pilitsis JG. The use of preoperative psychological evaluations to predict spinal cord stimulation success: Our experience and a review of the literature. Neuromodulation 2016; 19:429-436.

598. Blackburn DR, Romers CC, Copeland 
LA, Lynch W, Nguyen DD, Zeber JE, Hoffman MR. Presurgical psychological assessments as correlates of effectiveness of spinal cord stimulation for chronic pain reduction. Neuromodulation 2016; 19:422-428.

599. Fischer B, Murphy Y, Kurdyak P, Goldner EM. Depression - A major but neglected consequence contributing to the health toll from prescription opioids? Psychiatry Res 2016; 243:331-334.

6oo. Elbinoune I, Amine B, Shyen S, Gueddari S, Abouqal R, Hajjaj-Hassouni N. Chronic neck pain and anxiety-depression: Prevalence and associated risk factors. Pan Afr Med J 2016; 24:89.

6o1. Blozik E, Laptinskaya D, Herrmann-Lingen $\mathrm{C}$, Schaefer $\mathrm{H}$, Kochen MM, Himmel W, Scherer M. Depression and anxiety as major determinants of neck pain: A cross-sectional study in general practice. BMC Musculoskelet Disord 2009; 10:13.

602. Dimitriadis Z, Kapreli E, Strimpakos N Oldham J. Do psychological states associate with pain and disability in chronic neck pain patients? J Back Musculoskelet Rehabil 2015; 28:797-802.

603. Doleys DM, Brown J. MMPI profiles as an outcome 'predictor' in the treatment of non-cancer pain patients utilizing intraspinal opioid therapy. Neuromodulation 2001; 4:93-97.

604. Sehgal N, Manchikant L, Smith HS. Prescription opioid abuse in chronic pain: A review of opioid abuse predictors and strategies to curb opioid abuse. Pain Physician 2012; 15:ES67-ES92.

605. Nafziger AN, Bertino JS. Utility and application of urine drug testing in chronic pain management with opioids. Clin J Pain 2009; 25:73-79.

606. Rubinstein SM, Pool JJ, van Tulder MW, Riphagen II, de Vet HC. A systematic review of the diagnostic accuracy of provocative tests of the neck for diagnosing cervical radiculopathy. Eur Spine ] 2007; 16:307-319.

607. Laslett M, McDonald B, Tropp H, Aprill $\mathrm{CN}$, Oberg B. Agreement between diagnoses reached by clinical examination and available reference standards: A prospective study of 216 patients with lumbopelvic pain. BMC Musculoskelet Disord 2005; 6:28.

608. Petersen T, Olsen S, Laslett M, Thorsen H, Manniche C, Ekdahl C, Jacobsen S. Inter-tester reliability of a new diagnostic classification system for patients with non-specific low back pain. Aust J Physiother 2004; 50:85-94.
609. Sandmark H, Nisell R. Validity of five common manual neck pain provoking tests. Scand J Rehabil Med 1995; 27:131-136.

610. Carragee EJ. Validity of self-reported history in patients with acute back or neck pain after motor vehicle accidents. Spine ] 2008; 8:311-319.

611. Manchikanti L, Hirsch JA, Datta S, Falco FJE. Low back and lumbar radicular pain. In: Manchikanti L, Christo PJ, Trescot AM, Falco FJE (eds). Clinical Aspects of Pain Medicine and Interventional Pain Management: A Comprehensive Review. ASIPP Publishing, Paducah, KY, 2011, pp 87-114.

612. Manchikanti L, Falco FJE, Benyamin RM. Neck and cervical radicular pain. In: Manchikanti L, Christo PJ, Trescot AM, Falco FJE (eds). Clinical Aspects of Pain Medicine and Interventional Pain Management: A Comprehensive Review. ASIPP Publishing, Paducah, KY, 2011, pp 35-60.

613. Manchikanti L, Singh V, Datta S. Thoracic and chest wall pain and radicular pain. In: Manchikanti L, Christo PJ, Trescot AM, Falco FJE (eds). Clinical Aspects of Pain Medicine and Interventional Pain Management: A Comprehensive Review. ASIPP Publishing, Paducah, KY, 2011, pp 61-86.

614. Tong HC. Specificity of needle electromyography for lumbar radiculopathy in 55- to 79-yr-old subjects with low back pain and sciatica without stenosis. Am J Phys Med Rehabil 2011; 90:233-238.

615. Chiodo A, Haig AJ, Yamakawa KS, Quint D, Tong $H$, Choksi VR. Needle EMG has a lower false positive rate than MRI in asymptomatic older adults being evaluated for lumbar spinal stenosis. Clin Neurophysiol 2007; 118:751-756.

616. Haig AJ, Geisser ME, Tong HC, Yamakawa KS, Quint DJ, Hoff JT, Chiodo A, Miner JA, Phalke VV. Electromyographic and magnetic resonance imaging to predict lumbar stenosis, low-back pain, and no back symptoms. J Bone Joint Surg Am 2007; 89:358-366.

617. Haig AJ, Tong HC, Yamakawa KS, Quint DJ, Hoff JT, Chiodo A, Miner JA, Choksi VR, Geisser ME, Parres CM. Spinal stenosis, back pain, or no symptoms at all? A masked study comparing radiologic and electrodiagnostic diagnoses to the clinical impression. Arch Phys Med Rehabil 2006; 87:897-903.

618. Tong HC. Incremental ability of needle electromyography to detect radiculopathy in patients with radiating low back pain using different diagnostic criteria. Arch Phys Med Rehabil 2012;
93:990-992.

619. Ackerman SJ, Steinberg EP, Bryan RN, BenDebba M, Long DM. Patient characteristics associated with diagnostic imaging evaluation of persistent low back problems. Spine (Phila Pa 1976) 1997; 22:1634-1640; discussion 1641.

620. Rollins G. Rapid MRI no better than xray in the treatment of low back pain. Rep Med Guidel Outcomes Res 2003; 14:1, 6-7.

621. Boden SD, Swanson AL. An assessment of the early management of spine problems and appropriateness of diagnostic imaging utilization. Phys Med Rehabil Clin N Am 1998; 9:411-417, viii.

622. Jensen MC, Brant-Zawadzki MN, Obuchowski N, Modic MT, Malkasian D, Ross JS. Magnetic resonance imaging of the lumbar spine in people without back pain. N Engl J Med 1994; 331:69-73.

623. Powell MC, Wilson M, Szypryt P, Symonds EM, Worthington BS. Prevalence of lumbar disc degeneration observed by magnetic resonance in symptomless women. Lancet 1986; 2:1366-1367.

624. Boden SD, Davis DO, Dina TS, Patronas NJ, Wiesel SW. Abnormal magneticresonance scans of the lumbar spine in asymptomatic subjects. A prospective investigation. J Bone Joint Surg Am 1990; 72:403-408.

625. Takatalo J, Karppinen J, Niinimäki J, Taimela S, Näyhä S, Järvelin MR, Kyllönen E, Tervonen O. Prevalence of degenerative imaging findings in lumbar magnetic resonance imaging among young adults. Spine (Phila Pa 1976) 2009; 34:1716-1721.

626. Okada E, Matsumoto M, Ichihara D, Chiba K, Toyama Y, Fujiwara H, Momoshima S, Nishiwaki Y, Hashimoto T, Ogawa J, Watanabe M, Takahata T. Aging of the cervical spine in healthy volunteers: A 10-year longitudinal magnetic resonance imaging study. Spine (Phila Pa 1976) 2009; 34:706-712.

627. Wood KB, Blair JM, Aepple DM, Schendel MJ, Garvey TA, Gundry CR, Heithoff KB. The natural history of asymptomatic thoracic disc herniations. Spine (Phila $\mathrm{Pa}$ 1976) 1997; 22:525-529; discussion 529-530.

628. Okada E, Matsumoto M, Fujiwara $H$, Toyama Y. Disc degeneration of cervical spine on MRI in patients with lumbar disc herniation: Comparison study with asymptomatic volunteers. Eur Spine J 2011; 20:585-591.

629. Lebow RL, Adogwa O, Parker SL, Sharma A, Cheng J, McGirt MJ. Asymptom- 
atic same-site recurrent disc herniation after lumbar discectomy: Results of a prospective longitudinal study with 2-year serial imaging. Spine (Phila $\mathrm{Pa}$ 1976) 2011; 36:2147-2151.

630. Matsumoto $M$, Okada $E$, Ichihara $D$, Chiba K, Toyama Y, Fujiwara H, Momoshima S, Nishiwaki Y, Takahata T. Modic changes in the cervical spine: Prospective 10-year follow-up study in asymptomatic subjects. J Bone Joint Surg $\mathrm{Br}$ 2012; 94:678-683.

631. Matsumoto $M$, Okada E, Ichihara $D$, Chiba K, Toyama Y, Fujiwara H, Momoshima S, Nishiwaki $Y$, Hashimoto T, Inoue T, Watanabe M, Takahata T. Prospective ten-year follow-up study comparing patients with whiplash-associated disorders and asymptomatic subjects using magnetic resonance imaging. Spine (Phila Pa 1976) 2010; 35:1684-1690.

632. Owens DK, Qaseem A, Chou R, Shekelle P; Clinical Guidelines Committee of the American College of Physicians. High-value, cost-conscious health care: Concepts for clinicians to evaluate the benefits harms, and costs of medical interventions. Ann Intern Med 2011; 154:174-180.

633. Qaseem A, Alguire P, Dallas $P$, Feinberg LE, Fitzgerald FT, Horwitch C, Humphrey L, LeBlond R, Moyer D, Wiese JG, Weinberger S. Appropriate use of screening and diagnostic tests to foster high-value, cost-conscious care. Ann Intern Med 2012; 156:147-149.

634. Summaries for patients. Radiology tests for patients with low back pain: Highvalue health care advice from the American College of Physicians. Ann Intern Med 2011; 154:136.

635. Chou R, Qaseem A, Owens DK, Shekelle P; Clinical Guidelines Committee of the American College of Physicians. Diagnostic imaging for low back pain: Advice for high-value health care from the American College of Physicians. Ann Intern Med 2011; 154:181-189.

636. Andersen JC. Is immediate imaging important in managing low back pain? J Athl Train 2011; 46:99-102.

637. Friedman BW, Chilstrom M, Bijur PE, Gallagher EJ. Diagnostic testing and treatment of low back pain in United States emergency departments: A national perspective. Spine (Phila Pa 1976) 2010; 35:E1406-E1411.

638. Tonosu J, Oka H, Matsudaira K, Higashikawa A, Okazaki H, Tanaka S. The relationship between findings on magnetic resonance imaging and previous history of low back pain. J Pain Res 2017; 10:47-52.

639. Boos N, Semmer N, Elfering A, Schade V, Gal I, Zanetti M, Kissling R, Buchegger N, Hodler J, Main CJ. Natural history of individuals with asymptomatic disc abnormalities in magnetic resonance imaging: Predictors of low back painrelated medical consultation and work incapacity. Spine (Phila Pa 1976) 2000; 25:1484-1492.

640. Bouche KG, Vanovermeire O, Stevens VK, Coorevits PL, Caemaert JJ, Cambier DC, Verstraete K, Vanderstraeten GG, Danneels LA. Computed tomographic analysis of the quality of trunk muscles in asymptomatic and symptomatic lumbar discectomy patients. BMC Musculoskelet Disord 2011; 12:65.

641. Kraus CN, Baldwin AT, Curro FA, McAllister RG Jr. Clinical implications of patient-provider agreements in opioid prescribing. Curr Drug Saf 2015; 10:159-164.

642. Atluri SL, Akbik H, Sudarshan G. Prevention of opioid abuse in chronic noncancer pain: An algorithmic, evidencebased approach. Pain Physician 2012; 15:ES177-ES189.

643. Turk DC, Swanson KS, Gatchel RJ. Predicting opioid misuse by chronic pain patients: A systematic review and literature synthesis. Clin J Pain 2008; 24:497-508.

644. Passik SD, Kirsh KL, Whitcomb L, Portenoy RK, Katz NP, Kleinman L, Dodd SL, Schein JR. A new tool to assess and document pain outcomes in chronic pain patients receiving opioid therapy. Clin Ther 2004; 26:552-561.

645. Manchikanti L, Atluri S, Trescot AM, Giordano J. Monitoring opioid adherence in chronic pain patients: Tools, techniques, and utility. Pain Physician 2008; 11:S155-S180.

646. Højsted J, Sjøgren P. Addiction to opioids in chronic pain patients: A literature review. Eur J Pain 2007; 11:490-518.

647. Scheiderman N, Ironson G, Siegel SD. Stress and health: Psychological, behavioral, and biological determinants. Annu Rev Clin Psychol 2005; 1:607-628.

648. Stanton AL, Revenson TA, Tennen H. Health psychology: Psychological adjustment to chronic disease. Annu Rev Psychol 2007; 58:565-592.

649. Skinner EA, Zimmer-Gembeck MJ. The development of coping. Annu Rev Psychol 2007; 58:119-144.

650. Taylor SE, Stanton AL. Coping resourc- es, coping processes, and mental health. Annu Rev Clin Psychol 2007; 3:377-401.

651. Farrar JT. What is clinically meaningful: Outcome measures in pain clinical trials. Clin J Pain 2000; 16:S106-S112.

652. Salaffi F, Stancati A, Silvestri CA, Ciapetti A, Grassi W. Minimal clinically important changes in chronic musculoskeletal pain intensity measured on a numerical rating scale. Eur J Pain 2004; 8:283-291.

653. Bombardier C. Outcome assessments in the evaluation of treatment of spinal disorders: Summary and general recommendations. Spine (Phila Pa 1976) 2000; 25:3100-3103.

654. Hagg O, Fritzell P, Nordwall A. The clinical importance of changes in outcome scores after treatment for chronic low back pain. Eur Spine ] 2003; 12:12-20.

655. Manchikanti L, Hirsch JA, Cohen SP, Heavner JE, Falco FJE, Diwan S, Boswell MV, Candido KD, Onyewu O, Zhu J, Sehgal N, Kaye AD, Benyamin RM, Helm II S, Singh V, Datta S, Abdi S, Christo PJ, Hameed $H$, Hameed M, Vallejo R, Pampati V, Racz GB, Raj PP. Assessment of methodologic quality of randomized trials of interventional techniques: Development of an interventional pain management specific instrument. Pain Physician 2014; 17:E263-E290.

656. Manchikanti L, Hirsch JA, Heavner JE, Cohen SP, Benyamin RM, Sehgal N, Falco FJE, Vallejo R, Onyewu O, Zhu J, Kaye AD, Boswell MV, Helm II S, Candido KD, Diwan S, Simopoulos TT, Singh V, Pampati V, Racz GB, Raj PP. Development of an interventional pain management specific instrument for methodologic quality assessment of nonrandomized studies of interventional techniques. Pain Physician 2014; 17:E291-E317.

657. Education and Assessment for Overdose Prevention: A Review of the Clinical Evidence and Guidelines [Internet]. Ottawa (ON): Canadian Agency for Drugs and Technologies in Health; 2015 Sep 24.

658. Pedersen L, Borchgrevink PC, Breivik HP, Fredheim OM. A randomized, double-blind, double-dummy comparison of short- and long-acting dihydrocodeine in chronic non-malignant pain. Pain 2014; 155:881-888.

659. US Department of Health and Human Services, Food and Drug Administration goal of label changes: Better prescribing, safer use of opioids. Silver Spring, MD, 2013.

66o. Food and Drug Administration. FDA blueprint for prescriber education for extended-release and long-acting opi- 
oid analgesics. Silver Spring, MD, US Department of Health and Human Services, Food and Drug Administration, 2014.

661. Manchikanti L, Singh V, Caraway DL, Benyamin RM. Breakthrough pain in chronic non-cancer pain: Fact, fiction, or abuse. Pain Physician 2011; 14:E103-E117.

662. Agency Medical Directors Group. Interagency Guidelines on Opioid Dosing for Chronic Non-cancer Pain: An educational aid to improve care and safety with opioid therapy. 2010 Update.

www.agencymeddirectors.wa.gov/Files/ OpioidGdline.pdf

663. Gomes T, Juurlink DN, Dhalla IA, MailisGagnon A, Paterson JM, Mamdani MM. Trends in opioid use and dosing among socio-economically disadvantaged patients. Open Med 2011; 5:E13-E22.

664. Braden JB, Fan MY, Edlund MJ, Martin BC, DeVries A, Sullivan MD. Trends in use of opioids by noncancer pain type 2000-2005 among Arkansas Medicaid and HealthCore enrollees: Results from the TROUP study. J Pain 2008; 9:1026-1035.

665. Rome JD, Townsend CO, Bruce BK, Sletten CD, Luedtke CA, Hodgson JE. Chronic noncancer pain rehabilitation with opioid withdrawal: Comparison of treatment outcomes based on opioid use status at admission. Mayo Clinic Proceedings 2004; 79:759-768.

666. Pascual MLG, Fleming RRB, Gana TJ, Vorsanger GJ. Open-label study of the safety and effectiveness of long-term therapy with extended-release tramadol in the management of chronic nonmalignant pain. Curr Med Res Opin 2007; 23:2531-2542.

667. Walker JM, Farney RJ, Rhondeau SM, Boyle KM, Valentine K, Cloward TV, Shilling KC. Chronic opioid use is a risk factor for the development of central sleep apnea and ataxic breathing. J Clin Sleep Med 2007; 3:455-461.

668. Ballantyne JC, Mao J. Opioid therapy for chronic pain. N Engl J Med 2003; 349:1943-1953.

669. Alattar MA, Scharf SM. Opioid-associated central sleep apnea: A case series. Sleep Breath 2009; 13:201-206.

670. Braden JB, Russo J, Fan MY, Edlund MJ, Martin BC, DeVries A, Sullivan MD. Emergency department visits among recipients of chronic opioid therapy. Arch Intern Med 2010; 170:1425-1432.

671. Baron MJ, McDonald PW. Significant pain reduction in chronic pain patients after detoxification from high-dose opioids. J Opioid Manag 2006; 2:277-282.

672. Miller NS, Swiney T, Barkin RL. Effects of opioid prescription medication dependence and detoxification on pain perceptions and self-reports. Am J Ther 2006; 13:436-444.

673. Crisostomo RA, Schmidt JE, Hooten WM, Kerkvliet JL, Townsend CO, Bruce BK. Withdrawal of analgesic medication for chronic low-back pain patients: Improvement in outcomes of multidisciplinary rehabilitation regardless of surgical history. Am J Phys Med Rehabil 2008; 87:527-536.

674. Edlund MJ, Martin BC, Fan MY, Braden JB, Devries A, Sullivan MD. An analysis of heavy utilizers of opioids for chronic noncancer pain in the TROUP Study. J Pain Symptom Manage 2010; 40:279-289.

675. Katz N, Panas L, Kim M, Audet AD, Bilansky A, Eadie J, Kreiner P, Paillard FC, Thomas C, Carrow G. Usefulness of prescription monitoring programs for surveillance - analysis of Schedule II opioid prescription data in Massachusetts, 1996 - 2006. Pharmacoepidemiol Drug Safety 2010; 19:115-123.

676. Centers for Disease Control and Prevention (CDC). CDC grand rounds: Prescription drug overdoses - a U.S. epidemic. MMWR Morb Mortal Wkly Rep 2012; 61:10-13.

677. Cicero TJ, Wong G, Tian Y, Lynskey M, Todorov A, Isenberg K. Co-morbidity and utilization of medical services by pain patients receiving opioid medications: Data from an insurance claims database. Pain 2009; 144:20-27.

678. Lavin R, Park J. Depressive symptoms in community-dwelling older adults receiving opioid therapy for chronic pain. J Opioid Manag 2011; 7:309-319.

679. Fillingim RB, Doleys DM, Edwards RR, Lowery D. Clinical characteristics of chronic back pain as a function of gender and oral opioid use. Spine (Phila Pa 1976) 2003; 28:143-150.

680. Mahmud MA, Webster BS, Courtney TK, Matz S, Tacci JA, Christiani DC. Clinical management and the duration of disability for work-related low back pain. J Occup Environ Med 2000; 42:1178-1187.

681. Rhee Y, Taitel MS, Walker DR, Lau DT. Narcotic drug use among patients with lower back pain in employer health plans: A retrospective analysis of risk factors and health care services. Clin Ther 2007; 29:2603-2612.

682. Volinn E, Fargo JD, Fine PG. Opioid therapy for nonspecific low back pain and the outcome of chronic work loss. Pain 2009; 142:194-201.

683. Cifuentes M, Webster B, Genevay S, Pransky G. The course of opioid prescribing for a new episode of disabling low back pain: Opioid features and dose escalation. Pain 2010; 151:22-29.

684. Webster BS, Cifuentes M, Verma S, Pransky G. Geographic variation in opioid prescribing for acute, work-related, low back pain and associated factors: A multilevel analysis. Am J Ind Med 2009; 52:162-171.

685. Stover BD, Turner JA, Franklin G, Gluck JV, Fulton-Kehoe D, Sheppard L, Wickizer TM, Kaufman J, Egan K. Factors associated with early opioid prescription among workers with low back injuries. J Pain 2006; 7:718-725.

686. White AG, Birnbaum HG, Mareva MN, Daher M, Vallow S, Schein J, Katz N. Direct costs of opioid abuse in an insured population in the United States.] Manag Care Pharm 2005; 11:469-479.

687. Christopher MJ. It's time for bioethics to see chronic pain as an ethical issue. Am J Bioeth 2011; 11:3-4.

688. Cheatle MD, Savage SR. Informed consent in opioid therapy: A potential obligation and opportunity. J Pain Symptom Manage 2012; 44:105-116.

689. Pellegrini CA. Trust: The keystone of the patient-physician relationship. J Am Coll Surg 2016; S1072-7515(16)31566-6.

690. Buchman DZ, Ho A. What's trust got to do with it? Revisiting opioid contracts. J Med Ethics 2014; 40:673-677.

691. McGee S, Silverman RD. Treatment agreements, informed consent, and the role of state medical boards in opioid prescribing. Pain Med 2015; 16:25-29.

692. Arnold RM, Han PK, Seltzer D. Opioid contracts in chronic nonmalignant pain management: Objectives and uncertainties. Am J Med 2006; 119:292-296.

693. Payne R, Anderson E, Arnold R, Duensing L, Gilson A, Green C, Haywood C Jr, Passik S, Rich B, Robin L, Shuler N, Christopher M. A rose by any other name: Pain contracts/agreements. Am ] Bioeth 2010; 10:5-12.

694. Savage S. The patient-centered opioid treatment agreement. Am J Bioeth 2010; 10:18-19.

695. Fishman SM, Gallagher RM, McCarberg $\mathrm{BH}$. The opioid treatment agreement: A real-world perspective. Am J Bioeth 2010; 10:14-15.

696. Hahn MB. Patient compliance; where- 
fore art thou? Am J Bioeth 2010; 10:13-14.

697. Kirsh KL, Fishman SM. Multimodal approaches to optimize outcomes of chronic opioid therapy in the management of chronic pain. Pain Med 2011; 12:Si-Sil.

698. Tobin DG, Keough Forte K, Johnson McGee S. Breaking the pain contract: A better controlled-substance agreement for patients on chronic opioid therapy. Cleve Clin J Med 2016; 83:827-835.

699. Fishman SM, Mahajan G, Jung SW, Wilsey BL. The trilateral opioid contract. Bridging the7 pain clinic and the primary care physician through the opioid contract. J Pain Symptom Manage 2002; 24:335-344.

700. Keeping Radiation Exposures and Doses "As Low as Reasonably Achievable (ALARA)." G-129, Revision 1. Canadian Nuclear Safety Commission. October 2004.

http://nuclearsafety.gc.ca/pubs_catalogue/uploads/Gi2grevi_e.pdf

701. Preston KL, Jasinski DR, Testa M. Abuse potential and pharmacological comparison of tramadol and morphine. Drug Alcohol Depend 1991; 27:7-17.

702. Cicero TJ, Inciardi JA, Adams EH, Geller A, Senay EC, Woody GE, Woody GE, Muñoz A. Rates of abuse of tramadol remain unchanged with the introduction of new branded and generic products: Results of an abuse monitoring system, 1994-2004. Pharmacoepidemiol Drug Saf 2005; 14:851-859.

703. Dasgupta N, Kramer ED, Zalman MA, Carino S Jr, Smith MY, Haddox JD, Wright $C$ 4th. Association between non-medical and prescriptive usage of opioids. Drug Alcohol Depend 2006; 82:135-142.

704. Ayonrinde OT, Bridge DT. The rediscovery of methadone for cancer pain management. Med J Aust 2000; 173:536-540.

705. Perrin-Terrin A, Pathak A, Lapeyre-Mestre M. QT interval prolongation: Prevalence, risk factors and pharmacovigilance data among methadone-treated patients in France. Fundam Clin Pharmacol 2011; 25:503-510.

706. Modesto-Lowe V, Brooks D, Petry N. Methadone deaths: Risk factors in pain and addicted populations. J Gen Intern Med 2010; 25:305-309.

707. Mayet S, Gossop M, Lintzeris N, Markides V, Strang J. Methadone maintenance, QTc and torsade de pointes: Who needs an electrocardiogram and what is the prevalence of QTc prolongation? Drug Alcohol Rev 2011; 30:388-396.

708. Sims SA, Snow LA, Porucznik CA. Surveillance of methadone-related adverse drug events using multiple public health data sources. J Biomed Inform 2007; 40:382-389.

709. Gostick N, Allen J, Cranfield R, Currie J, Grillage M, Hildebrand PJ, James I. A comparison of the efficacy and adverse effects of controlled release dihydrocodeine and immediate release dihydrocodeine in the treatment of pain in osteoarthritis and chronic back pain. In: Twycross RG (ed). The Edinburgh Symposium on Pain Control and Medical Education. Royal Society of Medicine Pr, London, 1989, pp 137-143.
710. Hale ME, Fleischmann R, Salzman R, Wild J, Iwan T, Swanton RE, Kaiko RF, Lacouture PG. Efficacy and safety of controlled release versus immediate release oxycodone randomized, double blind evaluation in patients with chronic back pain. Clin J Pain 1999; 15:179-183.

711. Lloyd RS, Costello F, Eves MJ, James IGV, Miller AJ. The efficacy and tolerability of controlled-release dihydrocodeine tablets and combination dextropropoxyphene/paracetamol tablets in patients with severe osteoarthritis of the hips. Curr Med Res Opin 1992; 13:37-48.

712. Salzman RT, Roberts MS, Wild J, Fabian C, Reder RF, Goldenheim PD. Can a controlled release oral dose form of oxycodone be used as readily as an immediate release form for the purpose of titrating to stable pain control? J Pain Symptom Manage 1999; 18:271-279.

713. Seifert CF, Kennedy S. Meperidine is alive and well in the new millennium: Evaluation of meperidine usage patterns and frequency of adverse drug reactions. Pharmacotherapy 2004; 24:776-783.

714. Paulson DM, Kennedy DT, Donovick RA, Carpenter RL, Cherubini M, Techner L, Du W, Ma Y, Schmidt WK, Wallin B, Jackson D. Alvimopan: An oral, peripherally acting, mu-opioid receptor antagonist for the treatment of opioid induced bowel dysfunction: A 21-day treatmentrandomized clinical trial. J Pain 2005; 6:184-192.

715. Colson J, Helm S, Silver S. Office-based opioid dependence treatment. Pain Physician 2012; 15:ES231-ES236. 